\title{
Gold Catalysis Enabling Furan-Fused Cyclobutenes as a Platform toward Cross Cycloadditions
}

Shouzhi Zhang, ${ }^{+, \dagger}$ Aijie Tang, ${ }^{+, \dagger}$ Ruyu Xie, ${ }^{\dagger}$ Zhiqiang Zhao, ${ }^{\dagger}$ Jinzhong Yao, ${ }^{\ddagger}$ Maozhong Miao ${ }^{*, \dagger}$

${ }^{\dagger}$ Department of Chemistry, Key Laboratory of Surface \& Interface Science of Polymer Materials of Zhejiang Province, Zhejiang Sci-Tech University, Hangzhou, Zhejiang 310018 (P. R. China)

${ }^{\ddagger}$ College of Biological, Chemical Sciences and Engineering, Jiaxing University, Jiaxing 314001, People's Republic of China

${ }^{+}$These authors contributed equally.

E-mail:mmzok@zstu.edu.cn

\section{Supporting Information \\ List of contents}

1. General Methods

2. Optimization of the reaction conditions

3. Procedure and experiment data for compound 4

4. Procedure and experiment data for compound 5

5. Gram-scale synthesis of the products $4 a$ and $5 a$

6. Transformation of the compounds $4 a$ and $5 a$

7. The synthesis of compounds 11, 13, 15, 17, 19 and 21

8. X-ray diffraction analysis of 4a, 5a, 5h' and 21

9. Copies of ${ }^{1} \mathrm{H}$ NMR and ${ }^{13} \mathrm{C}$ NMR

S49-S189 


\section{General Methods}

NMR spectra were recorded on a Bruker AV-400 MHz spectrometer. The ${ }^{1} \mathrm{H}$ NMR (400 MHz) chemical shifts were reported in parts per million $(\delta)$ relative to internal standard TMS $(0 \mathrm{ppm})$. The coupling constants, $J$ values are reported in Hertz $(\mathrm{Hz}) .{ }^{1} \mathrm{H}$ and ${ }^{13} \mathrm{C}$ nuclear magnetic resonance (NMR) spectra were acquired at various field strengths as indicated, and were referenced to $\mathrm{CDCl}_{3}$ (0.00 and 77.00 ppm for ${ }^{1} \mathrm{H}$ and ${ }^{13} \mathrm{C}$, respectively) or acetone- $\mathrm{d}_{6}\left(2.05\right.$ and $29.8 \mathrm{ppm}$ for ${ }^{1} \mathrm{H}$ and ${ }^{13} \mathrm{C}$, respectively). High-resolution mass spectra (HRMS) were recorded on a Waters TOFMS GCT Premier using ESI ionization. Melting points were measured with WRR digital point apparatus purchased from Shanghai INESA Physico optiacal instrument Co.,Ltd (least count: $0.1{ }^{\circ} \mathrm{C}$; inaccuracy: rt-200 ${ }^{\circ} \mathrm{C}, \pm 1^{\circ} \mathrm{C} 200-300{ }^{\circ} \mathrm{C} \pm 2{ }^{\circ} \mathrm{C}$ ). All commercial reagents were used without additional purification and solvents were dried by standard methods when necessary. Petroleum ether refers to the fraction with boiling point in the range $60-90{ }^{\circ} \mathrm{C}$. All the temperatures are referred to the oil baths, cryogenic thermostatic baths or ice/water bath. All reactions were monitored by TLC with GF 254 silica gel coated plates. Flash column chromatography was carried out using 200-300 mesh silica gel. Gold catalysts $\mathrm{Au}_{2}($ rac-BINAP $) \mathrm{Cl}_{2}{ }^{1}, \mathrm{Au}_{2}(\mathrm{R}-\mathrm{BINAP}) \mathrm{Cl}_{2}{ }^{1}, \mathrm{AuPPh}_{3} \mathrm{Cl}^{2}, \mathrm{SPhosAuCl}^{3}$, $\mathrm{XPhosAuCl}{ }^{4}, t-\mathrm{BuXPhosAuCl}^{4}$, and $\mathrm{IPrAuCl}^{5}$ were prepared according to the literature procedure. The preparation of compounds $\mathbf{1}^{6}, \mathbf{2}^{7}, \mathbf{3}^{8}, \mathbf{1 2}^{9}, \mathbf{1 8}^{10}$, and $\mathbf{2 0}^{11}$ followed the literature procedure.

\section{Optimization of the reaction conditions}

(a) Procedure for initial attempt of 4a:

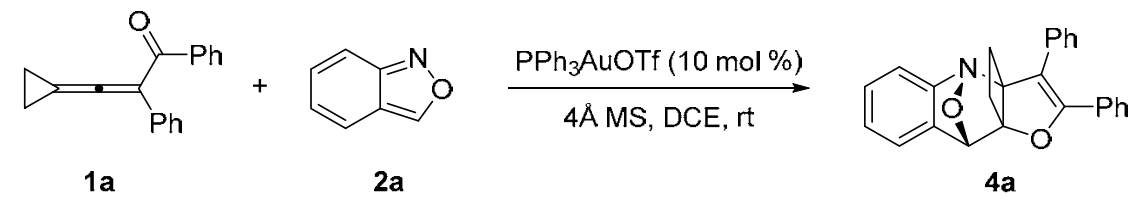

$\mathrm{AuPPh}_{3} \mathrm{OTf}(12.2 \mathrm{mg}, 0.020 \mathrm{mmol}, 10 \mathrm{~mol} \%)$ were dissolved in $2.0 \mathrm{~mL}$ of dry DCE in a dried vial under $\mathrm{N}_{2}$ atmosphere. Then, $4 \AA \mathrm{MS}(100 \mathrm{mg})$ and benzo[c]isoxazole 2a (60 $\mu \mathrm{L}, 0.60 \mathrm{mmol}, 3.0$ equiv) were added in this order and the reaction was stirred at room temperature. After being stirred for $5 \mathrm{~min}$, the 3-cyclopropylidene-1,2-diphenylprop-2-en-1-one 1a (49.1 mg, $0.200 \mathrm{mmol}, 1.0 \mathrm{equiv}$ ) was added. After being stirred for 12 hours, the reaction was determined by TLC. After completion, the solution was removed under reduced pressure, and purified by flash silica gel chromatography to afford product 4a which was obtained in 36\% yield.

Table S1. Optimization of the Reaction Conditions for the Formation of $\mathbf{4 a}$ 


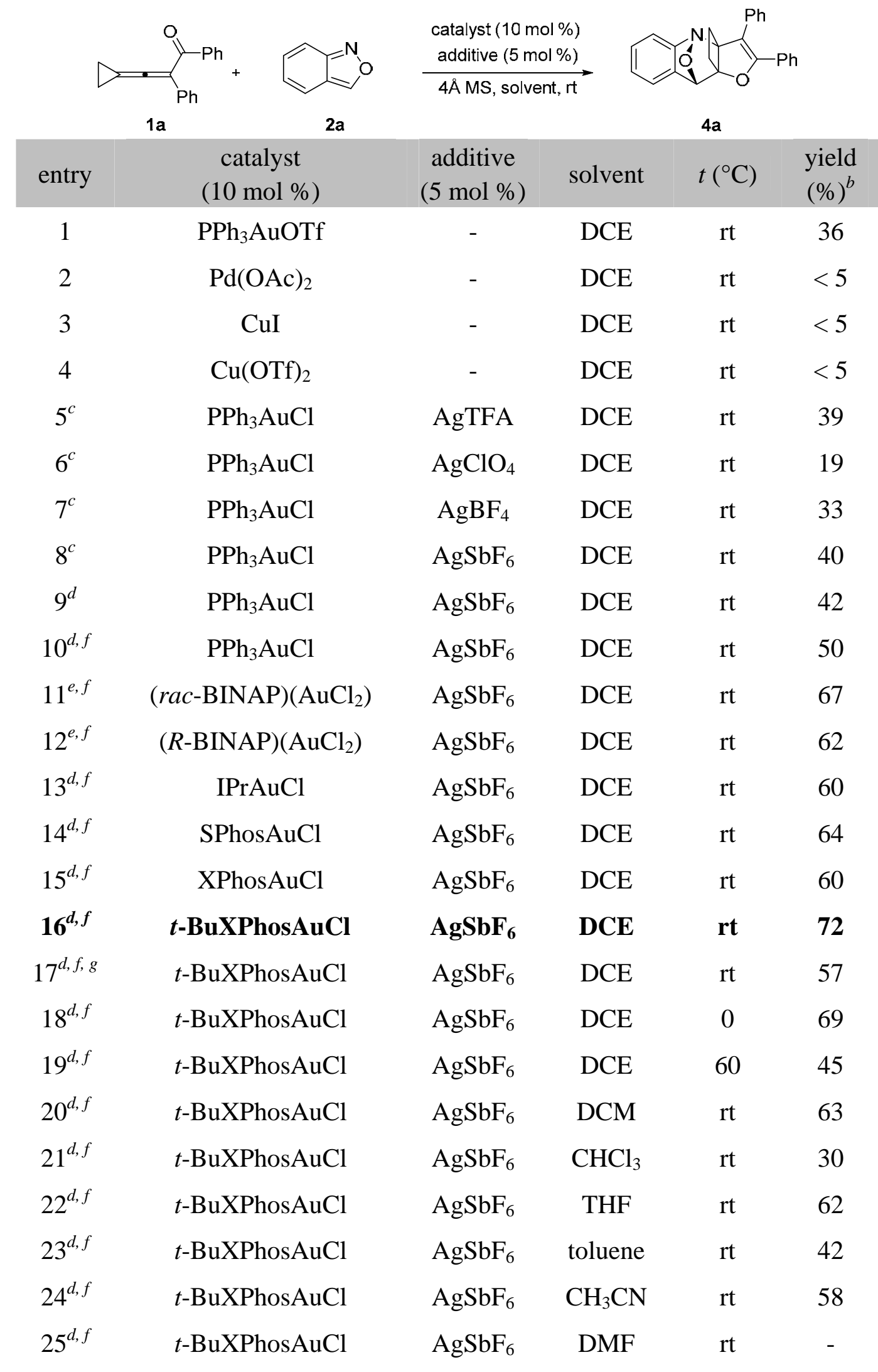

${ }^{a}$ Unless otherwise stated, the reaction was carried out using 1a $(0.2 \mathrm{mmol})$, 2a (0.6 mmol), $100 \mathrm{mg}$ of $4 \AA \mathrm{MS}, 10 \mathrm{~mol} \%$ of catalyst and $5 \mathrm{~mol} \%$ of additive under $\mathrm{N}_{2}$ atmosphere. ${ }^{b}$ Isolated yield. ${ }^{c} 5 \mathrm{~mol} \%$ of catalyst was used. ${ }^{d} 2 \mathrm{~mol} \%$ of catalyst was used. ${ }^{e} 1 \mathrm{~mol} \%$ of catalyst was used. ${ }^{f} 15 \mathrm{~mol} \%$ of additive was 
used. ${ }^{g} 0.3 \mathrm{mmol}$ of $\mathbf{2 a}$ was used.

(b) Procedure for initial attempt of 5a:

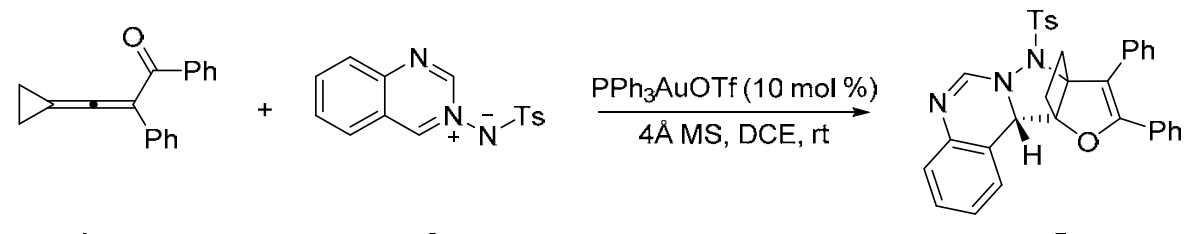

$1 \mathbf{a}$

$3 \mathbf{a}$

$5 a$

$\mathrm{AuPPh}_{3} \mathrm{OTf}$ (11.8 mg, $19.4 \mu \mathrm{mol}, 10 \mathrm{~mol} \%$ ) were dissolved in $2.0 \mathrm{~mL}$ of dry THF in a dried vial under $\mathrm{N}_{2}$ atmosphere. Then, $4 \AA$ MS (100 mg) and quinazolin-3-ium-3-yl(tosyl)amide 3a (66.1 mg, $0.221 \mathrm{mmol}, 1.1$ equiv) were added in this order and the reaction was stirred at room temperature. After being stirred for $5 \mathrm{~min}$, the 3-cyclopropylidene-1,2-diphenylprop-2-en-1-one 1a (49.5 mg, $0.201 \mathrm{mmol}, 1.0$ equiv) was added. After being stirred for 18 hours, the reaction was determined by TLC. After completion, the solution was removed under reduced pressure, and purified by flash silica gel chromatography to afford major product 5a in 40\% yield with $d r=8: 1$.

Table S2. Optimization of the Reaction Conditions for the Formation of 5a

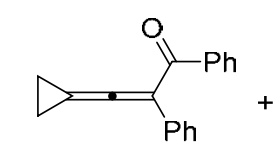

$1 \mathbf{a}$

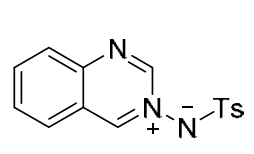

$3 \mathbf{a}$

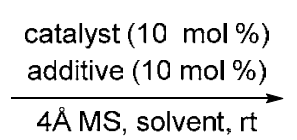

$4 \AA \mathrm{MS}$, solvent, $\mathrm{rt}$

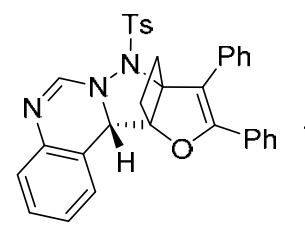

$5 a$

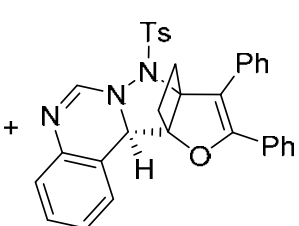

5a'

$\begin{array}{ccccccc}\text { entry } & \begin{array}{c}\text { catalyst } \\ (10 \mathrm{~mol} \%)\end{array} & \begin{array}{c}\text { additive } \\ (10 \mathrm{~mol} \%)\end{array} & \begin{array}{c}t \\ \text { solvent }\end{array} & \begin{array}{c}\text { yield } \\ \left({ }^{\circ} \mathrm{C}\right)\end{array} & \begin{array}{c}\text { (\%) })^{b, c} \\ \text { selectivity }^{d}\end{array} \\ 2 & \mathrm{PPh}_{3} \mathrm{AuOTf} & - & \mathrm{THF} & \mathrm{rt} & 40 & 8: 1 \\ 3 & \mathrm{Pd}(\mathrm{OAc})_{2} & - & \mathrm{THF} & \mathrm{rt} & <5 & - \\ 4 & \mathrm{CuI} & - & \mathrm{THF} & \mathrm{rt} & 38 & 7: 1 \\ 5 & \mathrm{Cu}(\mathrm{OTf})_{2} & - & \mathrm{THF} & \mathrm{rt} & <5 & - \\ 6 & \mathrm{PPh}_{3} \mathrm{AuCl} & \mathrm{AgOAc} & \mathrm{THF} & \mathrm{rt} & 41 & 8: 1 \\ 7 & \mathrm{PPh}_{3} \mathrm{AuCl} & \mathrm{AgNTf}_{2} & \mathrm{THF} & \mathrm{rt} & 43 & 7: 1 \\ 8 & \mathrm{PPh}_{3} \mathrm{AuCl} & \mathrm{AgClO}_{4} & \mathrm{THF} & \mathrm{rt} & 67 & 8: 1 \\ 9^{e} & \mathrm{PPh}_{3} \mathrm{AuCl} & \mathrm{AgSbF}_{6} & \mathrm{THF} & \mathrm{rt} & 80 & 8: 1 \\ 10^{e} & \mathrm{PPh}_{3} \mathrm{AuCl} & \mathrm{AgSbF}_{6} & \mathrm{THF} & \mathrm{rt} & 80 & 8: 1 \\ 11^{e} & \mathrm{PPh}_{3} \mathrm{AuCl} & \mathrm{AgSbF}_{6} & \mathrm{THF} & \mathrm{rt} & 61 & 7: 1 \\ 12^{e} & \mathrm{P}\left(p-\mathrm{ClC}_{6} \mathrm{H}_{4}\right)_{3} \mathrm{AuCl} & \mathrm{AgSbF}_{6} & \mathrm{THF} & \mathrm{rt} & 76 & 8: 1\end{array}$




$\begin{array}{lcccccr}13^{e} & \mathrm{P}\left(2,4,6-\mathrm{Me}_{3} \mathrm{C}_{6} \mathrm{H}_{2}\right)_{3} \mathrm{AuCl} & \mathrm{AgSbF}_{6} & \mathrm{THF} & \mathrm{rt} & 70 & 8: 1 \\ 14^{f} & \left(\text { rac-BINAP }(\mathrm{AuCl})_{2}\right. & \mathrm{AgSbF}_{6} & \mathrm{THF} & \mathrm{rt} & 79 & 8: 1 \\ 15^{e} & \mathrm{SPhosAuCl} & \mathrm{AgSbF}_{6} & \mathrm{THF} & \mathrm{rt} & 66 & 8: 1 \\ 16^{e} & \mathrm{IPrAuCl} & \mathrm{AgSbF}_{6} & \mathrm{THF} & \mathrm{rt} & 73 & 8: 1 \\ 17^{f} & \mathrm{PPh}_{3} \mathrm{AuCl} & \mathrm{AgSbF}_{6} & \mathrm{THF} & \mathrm{rt} & 80 & 8: 1 \\ 18^{f} & \mathrm{PPh}_{3} \mathrm{AuCl} & \mathrm{AgSbF}_{6} & \mathrm{CyH} & \mathrm{rt} & <5 & - \\ 19^{f} & \mathrm{PPh}_{3} \mathrm{AuCl} & \mathrm{AgSbF}_{6} & \mathrm{DMSO} & \mathrm{rt} & <5 & - \\ 20^{f} & \mathrm{PPh}_{3} \mathrm{AuCl} & \mathrm{AgSbF}_{6} & \text { o-DCB } & \mathrm{rt} & 58 & 3: 1 \\ 21^{f} & \mathrm{PPh}_{3} \mathrm{AuCl} & \mathrm{AgSbF}_{6} & \mathrm{DCM} & \mathrm{rt} & 62 & 4: 1 \\ 22^{f} & \mathrm{PPh}_{3} \mathrm{AuCl} & \mathrm{AgSbF}_{6} & \mathrm{dioxane} & \mathrm{rt} & 80 & 9: 1 \\ 23^{f} & \mathbf{P P h}_{3} \mathrm{AuCl} & \mathrm{AgSbF}_{6} & \mathbf{E A} & \mathbf{r t} & \mathbf{8 3} \mathbf{( 8 2})^{\mathbf{g}} & \mathbf{9}: \mathbf{1} \\ 24 & - & \mathrm{AgSbF}_{6} & \text { EA } & \mathrm{rt} & 77 & 9: 1 \\ 25^{f} & \mathrm{PPh}_{3} \mathrm{AuCl} & \mathrm{AgSbF}_{6} & \text { EA } & 0 & 64 & 13: 1 \\ 26^{f} & \mathrm{PPh}_{3} \mathrm{AuCl} & \mathrm{AgSbF}_{6} & \text { EA } & 60 & 81 & 7: 1\end{array}$

${ }^{a}$ Unless otherwise stated, the reaction was carried out using 1a $(0.2 \mathrm{mmol}), 2 \mathrm{a}(0.22 \mathrm{mmol})$, $100 \mathrm{mg}$ of $4 \AA \mathrm{MS}, 10 \mathrm{~mol} \%$ of catalyst and $5 \mathrm{~mol} \%$ of additive under $\mathrm{N}_{2}$ atmosphere. ${ }^{b}$ Yield of 5a. ${ }^{c 1} \mathrm{H}$ NMR yield using 2-methoxynaphalene as an internal standard. ${ }^{d}$ Ratio was determined by ${ }^{1} \mathrm{H}$ NMR analysis of the crude product. ${ }^{e} 2$ mol $\%$ of catalyst was used. ${ }^{f_{1}}$ mol \% of catalyst was used. ${ }^{g}$ Isolated yield.

\section{Procedure and experiment data for compound 4}

(a) General procedure (GP1):

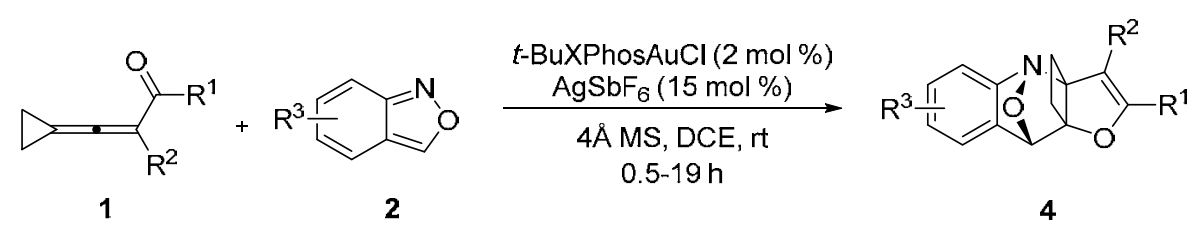

t-BuXPhosAuCl (4.0 $\mu \mathrm{mol}, 2 \mathrm{~mol} \%)$ and $\mathrm{AgSbF}_{6}(30.0 \mu \mathrm{mol}, 15 \mathrm{~mol} \%)$ were dissolved in $2.0 \mathrm{~mL}$ of dry DCE in a dried vial under $\mathrm{N}_{2}$ atmosphere. Then, 4Å MS (100 mg) and benzo[c]isoxazoles 2 (0.60 mmol, 3.0 equiv) were added in this order and the reaction was stirred at room temperature. After being stirred for $5 \mathrm{~min}$, the 3-cyclopropylideneprop-2-en-1-ones $\mathbf{1}$ (0.20 mmol, 1.0 equiv) was added. The reaction was determined by TLC (0.5-19 h). After completion, the solution was removed under reduced pressure, and purified by flash silica gel chromatography to afford product 4 .

(b) Experiment data:

(4S,9R)-2,3-diphenyl-9H-4,9-epoxy-3a,9a-ethanofuro[3,2-b]quinoline (4a) 


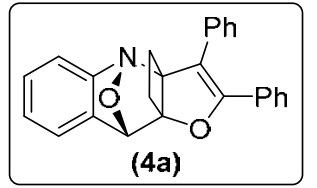

According to general procedure (GP1) with $t$-BuXPhosAuCl (2.7 mg, $4.1 \mu \mathrm{mol}$, $2 \mathrm{~mol} \%), \operatorname{AgSbF}_{6}(11.0 \mathrm{mg}, 32.0 \mu \mathrm{mol}, 15 \mathrm{~mol} \%)$, benzo[c]isoxazole $2 \mathrm{a}(60 \mu \mathrm{L}$, 0.60 mmol, 3.0 equiv), 3-cyclopropylidene-1,2-diphenylprop-2-en-1-one 1a (49.3 mg, $0.200 \mathrm{mmol}, 1.0$ equiv), $100 \mathrm{mg} 4 \AA \mathrm{MS}, 2.0 \mathrm{~mL}$ of dry DCE for 12 hours reaction. Purification by flash column chromatography provided 4a as a white solid (52.7 mg, 72\%); M.p. $115-117{ }^{\circ} \mathrm{C}$ (Petroleum ether/EtOAc); $\mathrm{R}_{f}=0.26$ (Petroleum ether/EtOAc $\left.=20 / 1\right) ;{ }^{1} \mathrm{H}$ NMR $(400$ $\left.\mathrm{MHz}, \mathrm{CDCl}_{3}\right): \delta$ 7.35-7.26 (m, 2H), 7.22-7.14 (m, 4H), 7.13-7.03 (m, 5H), 6.99-6.93 (m, 1H), 6.91 (d, $J=8.0 \mathrm{~Hz}, 2 \mathrm{H}), 5.54(\mathrm{~s}, 1 \mathrm{H}), 2.68-2.60(\mathrm{~m}, 2 \mathrm{H}), 2.59-2.47$ (m, 2H); ${ }^{13} \mathrm{C}$ NMR (100 MHz, $\left.\mathrm{CDCl}_{3}\right): \delta 159.0,152.0,137.9,131.9,131.3,129.5,128.2,128.1,127.3,127.2,126.2,126.2,122.2$, 116.5, 112.6, 89.4, 84.1, 28.9, 26.1; HRMS (ESI) m/z: $[\mathrm{M}+\mathrm{H}]^{+}$Calcd for $\mathrm{C}_{25} \mathrm{H}_{20} \mathrm{NO}_{2} 366.1489$; Found 366.1501.

(4R,9S)-6-chloro-2,3-diphenyl-9H-4,9-epoxy-3a,9a-ethanofuro[3,2-b]quinoline (4b)

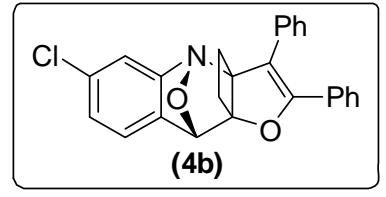

According to general procedure (GP1) with $t$-BuXPhosAuCl (2.4 mg, 3.7 $\mu \mathrm{mol}, 2 \mathrm{~mol} \%), \mathrm{AgSbF}_{6} \quad(11.2 \mathrm{mg}, 32.6 \mu \mathrm{mol}, 15 \mathrm{~mol} \%)$, 6-chlorobenzo[c]isoxazole $\mathbf{2 b} \quad(93.0 \mathrm{mg}, \quad 0.610 \mathrm{mmol}, 3.0$ equiv),

3-cyclopropylidene-1,2-diphenylprop-2-en-1-one 1a (49.8 mg, $0.204 \mathrm{mmol}, 1.0$ equiv), $100 \mathrm{mg} 4 \AA$ MS, $2.0 \mathrm{~mL}$ of dry DCE for 12 hours reaction. Purification by flash column chromatography provided 4b as a white solid (64.0 mg, 79\%); M.p. 148-150 ${ }^{\circ} \mathrm{C}$ (Petroleum ether/EtOAc); $\mathrm{R}_{f}=0.19$ $($ Petroleum ether/EtOAc $=20 / 1) ;{ }^{1} \mathrm{H}$ NMR $\left(400 \mathrm{MHz}, \mathrm{CDCl}_{3}\right): \delta 7.28(\mathrm{~d}, J=7.2 \mathrm{~Hz}, 1 \mathrm{H}), 7.24-7.16$ (m, 5H), 7.15-7.08 (m, 3H), 7.06-7.01 (m, 1H), 6.98 (d, J = 7.2 Hz, 2H), 6.91 (s, 1H), 5.53 (s, 1H), 2.68-2.60 (m, 2H), 2.59-2.47 (m, 2H); ${ }^{13} \mathrm{C}$ NMR (100 MHz, $\mathrm{CDCl}_{3}$ ): $\delta 159.0,153.6,136.7,133.0$, 131.7, 131.0, 129.7, 128.4, 128.3, 128.0, 127.3, 126.5, 126.2, 122.9, 117.3, 112.3, 89.2, 84.2, 83.9, 28.9, 26.0; HRMS (ESI) m/z: [M + H] Calcd for $\mathrm{C}_{25} \mathrm{H}_{19} \mathrm{ClNO}_{2}$ 400.1099; Found 400.1106.

\section{(4R,9S)-6-bromo-2,3-diphenyl-9H-4,9-epoxy-3a,9a-ethanofuro[3,2-b]quinoline (4c)}

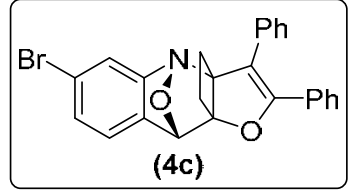

According to general procedure (GP1) with $t$-BuXPhosAuCl (2.7 mg, 4.1 $\mu \mathrm{mol}, 2 \mathrm{~mol} \%), \mathrm{AgSbF}_{6} \quad(10.1 \mathrm{mg}, 29.4 \mu \mathrm{mol}, 15 \mathrm{~mol} \%)$, 6-bromobenzo[c]isoxazole 2c (118.1 mg, $0.5965 \mathrm{mmol}, 3.0$ equiv), 3-cyclopropylidene-1,2-diphenylprop-2-en-1-one 1a (49.0 mg, $0.199 \mathrm{mmol}, 1.0$ equiv), $100 \mathrm{mg} 4 \AA$ MS, $2.0 \mathrm{~mL}$ of dry DCE for 12 hours reaction. Purification by flash column chromatography provided 4c as a white solid (61.8 mg, 70\%); M.p. 152-153 ${ }^{\circ} \mathrm{C}$ (Petroleum ether/EtOAc); $\mathrm{R}_{f}=0.21$ 
$($ Petroleum ether/EtOAc $=20 / 1) ;{ }^{1} \mathrm{H}$ NMR $\left(400 \mathrm{MHz}, \mathrm{CDCl}_{3}\right): \delta 7.29(\mathrm{~d}, J=7.4 \mathrm{~Hz}, 1 \mathrm{H}), 7.24-7.13$ (m, 7H), 7.10 (d, $J=7.6 \mathrm{~Hz}, 2 \mathrm{H}), 7.05$ (s, 1H), 6.98 (d, $J=7.6 \mathrm{~Hz}, 2 \mathrm{H}), 5.52$ (s, 1H), 2.68-2.60 (m, 2H), 2.59-2.47 (m, 2H); ${ }^{13} \mathrm{C}$ NMR (100 MHz, $\mathrm{CDCl}_{3}$ ): $\delta$ 159.0, 153.8, 137.2, 131.7, 131.0, 129.7, 129.1, 128.4, 128.3, 128.1, 127.3, 126.5, 123.4, 120.7, 120.1, 112.4, 89.1, 84.3, 84.0, 29.0, 26.1; HRMS (ESI) m/z: [M + H] $]^{+}$Calcd for $\mathrm{C}_{25} \mathrm{H}_{19} \mathrm{BrNO}_{2}$ 444.0594; Found 444.0599.

\section{(4R,9S)-6-nitro-2,3-diphenyl-9H-4,9-epoxy-3a,9a-ethanofuro[3,2-b]quinoline (4d)}

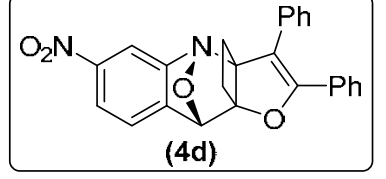

According to general procedure (GP1) with $t$-BuXPhosAuCl (2.5 mg, 3.8 $\mu \mathrm{mol}, 2 \mathrm{~mol} \%), \mathrm{AgSbF}_{6}$ (10.1 mg, $\left.29.4 \mu \mathrm{mol}, 15 \mathrm{~mol} \%\right)$, 6-nitrobenzo[c]isoxazole $\quad$ 2d $\quad(33.5 \mathrm{mg}, \quad 0.204 \mathrm{mmol}, \quad 1.0$ equiv),

3-cyclopropylidene-1,2-diphenylprop-2-en-1-one 1a (148.0 mg, 0.6016 mmol, 3.0 equiv), $100 \mathrm{mg}$ $4 \AA$ MS, $2.0 \mathrm{~mL}$ of dry DCE for 16 hours reaction. Purification by flash column chromatography provided 4d as a white solid (44.2 mg, 53\%); M.p. 140-142 ${ }^{\circ} \mathrm{C}$ (Petroleum ether/EtOAc); $\mathrm{R}_{f}=0.29$ $\left(\right.$ Petroleum ether/EtOAc = 20/1); ${ }^{1} \mathrm{H}$ NMR $\left(400 \mathrm{MHz}, \mathrm{CDCl}_{3}\right): \delta 8.03(\mathrm{~d}, J=8.0 \mathrm{~Hz}, 1 \mathrm{H}), 7.72$ (s, 1H), 7.54-7.43 (m, 1H), 7.33-7.24 (m, 1H), 7.23-7.13 (m, 5H), 7.09 (d, $J=5.6$ Hz, 2H), 6.99-6.88 (m, 2H), 5.66 (s, 1H), 2.76-2.65 (m, 2H), 2.64-2.52 (m, 2H); ${ }^{13} \mathrm{C}$ NMR (100 MHz, $\left.\mathrm{CDCl}_{3}\right): \delta 159.0$, 153.7, 147.4, 145.3, 131.2, 130.4, 129.9, 128.6, 128.4, 127.8, 127.3, 127.0, 122.5, 122.3, 112.0, 88.9, 84.4, 83.9, 29.0, 26.1; HRMS (ESI) m/z: $[\mathrm{M}+\mathrm{H}]^{+}$Calcd for $\mathrm{C}_{25} \mathrm{H}_{19} \mathrm{~N}_{2} \mathrm{O}_{4}$ 411.1339; Found 411.1343. methyl (4R,9S)-2,3-diphenyl-9H-4,9-epoxy-3a,9a-ethanofuro[3,2-b]quinoline-6-carboxylate (4e)

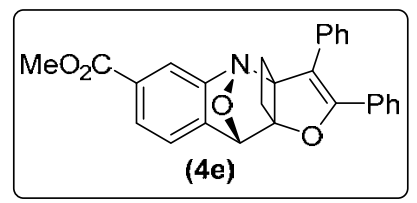

According to general procedure (GP1) with $t$-BuXPhosAuCl (2.4 mg, 3.7 $\mu \mathrm{mol}, 2 \mathrm{~mol} \%), \operatorname{AgSbF}_{6}$ (10.9 mg, $\left.31.7 \mu \mathrm{mol}, 15 \mathrm{~mol} \%\right)$, methyl benzo[c]isoxazole-6-carboxylate $2 \mathbf{e}$ (107.2 $\mathrm{mg}, 0.6050 \mathrm{mmol}, 3.0$ equiv),

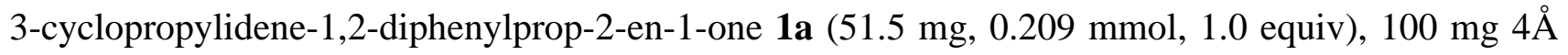
MS, $2.0 \mathrm{~mL}$ of dry DCE for 12 hours reaction. Purification by flash column chromatography provided 4e as a white solid (74.5 mg, 84\%); M.p. 90-91 ${ }^{\circ} \mathrm{C}$ (Petroleum ether/EtOAc); $\mathrm{R}_{f}=0.18$ (Petroleum ether/EtOAc = 20/1); ${ }^{1} \mathrm{H}$ NMR (400 MHz, $\left.\mathrm{CDCl}_{3}\right): \delta$ 7.90-7.72 (m, 1H), 7.55 (s, 1H), 7.39 (d, $J=7.6 \mathrm{~Hz}, 1 \mathrm{H}), 7.24-7.21(\mathrm{~m}, 1 \mathrm{H}), 7.20-7.12$ (m, 5H), 7.11-7.05 (m, 2H), 6.92 (d, $J=7.2$ $\mathrm{Hz}, 2 \mathrm{H}), 5.58$ (s, 1H), 3.82 (s, 3H), 2.70-2.61 (m, 2H), 2.60-2.50 (m, 2H); ${ }^{13} \mathrm{C}$ NMR (100 MHz, $\left.\mathrm{CDCl}_{3}\right): \delta 166.3,159.0,152.6,143.3,131.7,130.9,129.6,129.5,128.4,128.3,128.0,127.4,126.5$, 122.0, 117.4, 112.5, 89.2, 84.3, 84.1, 52.2, 29.1, 26.2; HRMS (ESI) m/z: $[M+H]^{+}$Calcd for $\mathrm{C}_{27} \mathrm{H}_{22} \mathrm{NO}_{4} 424.1543$; Found 424.1552. 


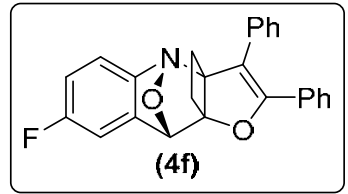

(4f)

According to general procedure (GP1) with $t$-BuXPhosAuCl (2.7 mg, 4.1 $\mu \mathrm{mol}, 2 \mathrm{~mol} \%), \mathrm{AgSbF}_{6}$ (11.2 mg, $\left.32.6 \mu \mathrm{mol}, 15 \mathrm{~mol} \%\right)$, 5-fluorobenzo[c]isoxazole 2 ff $(83.1 \mathrm{mg}, \quad 0.607 \mathrm{mmol}, \quad 3.0$ equiv),

3-cyclopropylidene-1,2-diphenylprop-2-en-1-one 1a (49.2 mg, $0.200 \mathrm{mmol}, 1.0$ equiv), $100 \mathrm{mg} 4 \AA$ MS, $2.0 \mathrm{~mL}$ of dry DCE for 12 hours reaction. Purification by flash column chromatography provided 4 f as a white solid (40.3 mg, 53\%); M.p. $138-139{ }^{\circ} \mathrm{C}$ (Petroleum ether/EtOAc); $\mathrm{R} f=0$. $19($ Petroleum ether/EtOAc $=20 / 1) ;{ }^{1} \mathrm{H}$ NMR $\left(400 \mathrm{MHz}, \mathrm{CDCl}_{3}\right): \delta$ 7.32-7.08 (m, 8H), 7.07-7.01 (m, 1H), 6.97 (d, $J=7.2 \mathrm{~Hz}, 2 \mathrm{H}), 6.90-6.83$ (m, 1H), 6.79-6.72 (m, 1H), 5.53 (s, 1H), 2.68-2.46 (m, 4H); ${ }^{13} \mathrm{C}$ NMR (100 MHz, $\left.\mathrm{CDCl}_{3}\right): \delta 161.0$ (d, $\left.J=243.9 \mathrm{~Hz}\right), 159.1,147.9,140.2$ (d, $\left.J=8.9 \mathrm{~Hz}\right), 131.8$, 131.1, 129.7, 128.4 (d, $J=5.9 \mathrm{~Hz}), 128.1,127.2,126.4,117.5,117.4,113.4$ (d, $J=23.6 \mathrm{~Hz}), 112.6$, 110.6, 110.3, 89.3, 84.3, 28.9, 26.1; HRMS (ESI) m/z: $[\mathrm{M}+\mathrm{H}]^{+}$Calcd for $\mathrm{C}_{25} \mathrm{H}_{19} \mathrm{FNO}_{2}$ 384.1394; Found 384.1401.

\section{(4R,9S)-7-chloro-2,3-diphenyl-9H-4,9-epoxy-3a,9a-ethanofuro[3,2-b]quinoline (4g)}

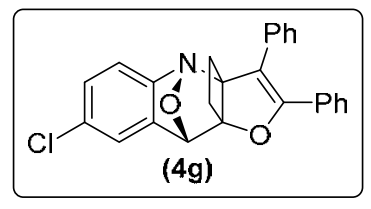

According to general procedure (GP1) with $t$-BuXPhosAuCl $(2.7 \mathrm{mg}, 4.1$ $\mu \mathrm{mol}, 2 \mathrm{~mol} \%), \mathrm{AgSbF}_{6}(11.0 \mathrm{mg}, 32.0 \mu \mathrm{mol}, 15 \mathrm{~mol} \%)$, 5-chlorobenzo[c]isoxazole $\mathbf{2 g} \quad(93.1 \mathrm{mg}, \quad 0.606 \mathrm{mmol}, 3.0$ equiv), 3-cyclopropylidene-1,2-diphenylprop-2-en-1-one 1a (49.8 mg, $0.202 \mathrm{mmol}, 1.0$ equiv), $100 \mathrm{mg} 4 \AA$ MS, $2.0 \mathrm{~mL}$ of dry DCE for 12 hours reaction. Purification by flash column chromatography provided $\mathbf{4 g}$ as a white solid (50.8 mg, 63\%); M.p. $125-127{ }^{\circ} \mathrm{C}$ (Petroleum ether/EtOAc); $\mathrm{R}_{f}=0.17$ $($ Petroleum ether/EtOAc $=20 / 1) ;{ }^{1} \mathrm{H}$ NMR (400 MHz, $\left.\mathrm{CDCl}_{3}\right): \delta$ 7.34-7.27 (m, 2H), 7.24-7.20 (m, 2H), 7.18-7.02 (m, 6H), 6.98-6.91 (m, 2H), 6.82 (d, $J=8.4$ Hz, 1H), 5.52 (s, 1H), 2.68-2.61 (m, 2H), 2.59-2.47 (m, 2H); ${ }^{13} \mathrm{C}$ NMR (100 MHz, $\left.\mathrm{CDCl}_{3}\right): \delta 159.2,150.8,140.0,131.8,131.7,131.1,129.7$, 128.45, 128.35, 128.1, 127.1, 126.4, 122.8, 117.6, 112.5, 89.3, 84.2, 84.1, 29.0, 26.1; HRMS (ESI) $\mathrm{m} / \mathrm{z}:[\mathrm{M}+\mathrm{H}]^{+}$Calcd for $\mathrm{C}_{25} \mathrm{H}_{19} \mathrm{ClNO}_{2}$ 400.1099; Found 400.1102 .

\section{(4R,9S)-7-bromo-2,3-diphenyl-9H-4,9-epoxy-3a,9a-ethanofuro[3,2-b]quinoline (4h)}

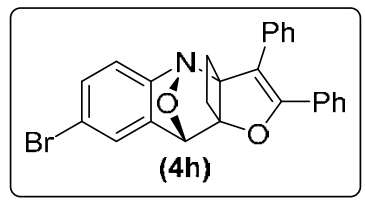

According to general procedure (GP1) with $t$-BuXPhosAuCl (2.7 mg, 4.1 $\mu \mathrm{mol}, 2 \mathrm{~mol} \%), \mathrm{AgSbF}_{6}(11.0 \mathrm{mg}, 32.0 \mu \mathrm{mol}, 15 \mathrm{~mol} \%)$, 5-bromobenzo[c]isoxazole $\mathbf{2 h}$ (119.3 $\mathrm{mg}, \quad 0.603 \mathrm{mmol}, \quad 3.0$ equiv), 3-cyclopropylidene-1,2-diphenylprop-2-en-1-one 1a (48.9 mg, $0.199 \mathrm{mmol}, 1.0$ equiv), $100 \mathrm{mg} 4 \AA$ 
MS, $2.0 \mathrm{~mL}$ of dry DCE for 12 hours reaction. Purification by flash column chromatography provided $4 \mathbf{h}$ as a white solid (57.4 mg, 65\%); M.p. 127-128 ${ }^{\circ} \mathrm{C}$ (Petroleum ether/EtOAc); $\mathrm{R}_{f}=0.21$ $\left(\right.$ Petroleum ether/EtOAc = 20/1); ${ }^{1} \mathrm{H}$ NMR (400 MHz, $\left.\mathrm{CDCl}_{3}\right): \delta$ 7.47-7.44 (m, 1H), 7.34-7.27 (m, 1H), 7.24-7.19 (m, 3H), 7.16-7.04 (m, 5H), 6.94 (d, $J=7.2 \mathrm{~Hz}, 2 \mathrm{H}), 6.76$ (d, $J=8.0 \mathrm{~Hz}, 1 \mathrm{H}), 5.52$ (s, 1H), 2.70-2.61 (m, 2H), 2.59-2.47 (m, 2H); ${ }^{13} \mathrm{C}$ NMR (100 MHz, $\left.\mathrm{CDCl}_{3}\right): \delta 159.2,151.4,140.4$, 131.7, 131.1, 130.1, 129.7, 128.5, 128.3, 128.1, 127.1, 126.4, 125.6, 119.5, 118.0, 112.4, 89.3, 84.1, 84.0, 29.0, 26.1; HRMS (ESI) m/z: $[\mathrm{M}+\mathrm{H}]^{+}$Calcd for $\mathrm{C}_{25} \mathrm{H}_{19} \mathrm{BrNO}_{2}$ 444.0594; Found 444.0601.

\section{(4R,9S)-2-(4-fluorophenyl)-3-phenyl-9H-4,9-epoxy-3a,9a-ethanofuro[3,2-b]quinoline (4i)}

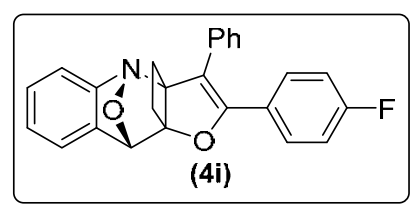

(4i)

According to general procedure with $t$-BuXPhosAuCl $(2.7 \mathrm{mg}, 4.1 \mu \mathrm{mol}, 2$ mol \%), $\operatorname{AgSbF}_{6}(10.7 \mathrm{mg}, 31.1 \mu \mathrm{mol}, 15 \mathrm{~mol} \%)$, benzo[c]isoxazole 2a $(60$ $\mu \mathrm{L}, \quad 0.60 \quad \mathrm{mmol}$, 3.0

equiv),

3-cyclopropylidene-1-(4-fluorophenyl)-2-phenylprop-2-en-1-one $\mathbf{1 b}$ (52.3 mg, 0.198 mmol, 1.0 equiv), $100 \mathrm{mg} 4 \AA \mathrm{MS}, 2.0 \mathrm{~mL}$ of dry DCE for 12 hours reaction. Purification by flash column chromatography provided $4 \mathbf{i}$ as a white solid (59.3 mg, 78\%); M.p. $145-147{ }^{\circ} \mathrm{C}$ (Petroleum ether/EtOAc); $\mathrm{R}_{f}=0.18$ (Petroleum ether/EtOAc = 20/1); ${ }^{1} \mathrm{H}$ NMR $\left(400 \mathrm{MHz}, \mathrm{CDCl}_{3}\right): \delta$ 7.34-7.27 (m, 1H), 7.22-7.03 (m, 7H), 6.99-6.93 (m, 1H), 6.92-6.81 (m, 4H), 5.54 (s, 1H), 2.68-2.59 (m, 2H), 2.58-2.49 (m, 2H); ${ }^{13} \mathrm{C}$ NMR (100 MHz, $\left.\mathrm{CDCl}_{3}\right): \delta 163.2$ (d, $\left.J=248.5 \mathrm{~Hz}\right), 157.8,152.1,138.0$, 131.8, 130.2 (d, $J=8.5 \mathrm{~Hz}), 128.4,127.3,126.4,126.2$, 122.2, 116.5, 115.4 (d, $J=21.7 \mathrm{~Hz}), 112.8$, 89.4, 84.1, 28.9, 26.0; HRMS (ESI) m/z: $[\mathrm{M}+\mathrm{H}]^{+}$Calcd for $\mathrm{C}_{25} \mathrm{H}_{19} \mathrm{FNO}_{2}$ 384.1394; Found 384.1400 .

\section{(4R,9S)-2-(4-chlorophenyl)-3-phenyl-9H-4,9-epoxy-3a,9a-ethanofuro[3,2-b]quinoline (4j)}

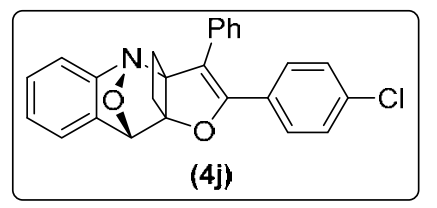

According to general procedure (GP1) with $t$-BuXPhosAuCl (2.4 mg, 3.6 $\mu \mathrm{mol}, 2 \mathrm{~mol} \%), \operatorname{AgSbF}_{6}$ (10.8 mg, $\left.31.4 \mu \mathrm{mol}, 15 \mathrm{~mol} \%\right)$, benzo[c]isoxazole $\quad 2 a \quad(60 \mu \mathrm{L}, \quad 0.60 \quad \mathrm{mmol}, \quad 3.0 \quad$ equiv $)$, 1-(4-chlorophenyl)-3-cyclopropylidene-2-phenylprop-2-en-1-one $\mathbf{1 b}$ (56.7 mg, $0.201 \mathrm{mmol}, 1.0$ equiv), $100 \mathrm{mg} 4 \AA \mathrm{MS}, 2.0 \mathrm{~mL}$ of dry DCE for 12 hours reaction. Purification by flash column chromatography provided $\mathbf{4 j}$ as a white solid (61.6 mg, 76\%); M.p. 130-132 ${ }^{\circ} \mathrm{C}$ (Petroleum ether/EtOAc); $\mathrm{R}_{f}=0.17$ (Petroleum ether/EtOAc = 20/1); ${ }^{1} \mathrm{H}$ NMR $\left(400 \mathrm{MHz}, \mathrm{CDCl}_{3}\right): \delta$ 7.32-7.27 (m, 1H), 7.22-7.09 (m, 7H), 7.08-7.03 (m, 2H), 6.97-6.93 (m, 1H), 6.86 (d, J = 8.4 Hz, 2H), 5.54 (s, 1H), 2.68-2.59 (m, 2H), 2.58-2.49 (m, 2H); ${ }^{13} \mathrm{C}$ NMR (100 MHz, $\left.\mathrm{CDCl}_{3}\right): \delta$ 157.5, 152.0, 137.9, 
135.4, 131.7, 129.6, 129.5, 128.6, 128.4, 127.35, 127.29, 126.5, 126.3, 122.2, 116.5, 113.2, 89.5, 84.1, 28.9. 26.0; HRMS (ESI) m/z: $[\mathrm{M}+\mathrm{H}]^{+}$Calcd for $\mathrm{C}_{25} \mathrm{H}_{19} \mathrm{ClNO}_{2}$ 400.1099; Found 400.1103. (4R,9S)-2-(4-bromophenyl)-3-phenyl-9H-4,9-epoxy-3a,9a-ethanofuro[3,2-b]quinoline (4k)

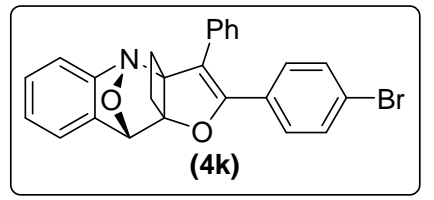

According to general procedure (GP1) with $t$-BuXPhosAuCl (2.8 mg, 4.3 $\mu \mathrm{mol}, 2 \mathrm{~mol} \%), \operatorname{AgSbF}_{6}$ (11.1 mg, $\left.32.3 \mu \mathrm{mol}, 15 \mathrm{~mol} \%\right)$, benzo[c]isoxazole $\quad 2 a \quad(60 \mu \mathrm{L}, \quad 0.60 \quad \mathrm{mmol}, \quad 3.0 \quad$ equiv $)$, 1-(4-bromophenyl)-3-cyclopropylidene-2-phenylprop-2-en-1-one 1d (65.4 mg, $0.201 \mathrm{mmol}, 1.0$ equiv), $100 \mathrm{mg} 4 \AA \mathrm{MS}, 2.0 \mathrm{~mL}$ of dry DCE for 12 hours reaction. Purification by flash column chromatography provided $\mathbf{4 k}$ as a white solid (61.1 mg, 68\%); M.p. 130-131 ${ }^{\circ} \mathrm{C}$ (Petroleum ether/EtOAc); $\mathrm{R}_{f}=0.12$ (Petroleum ether/EtOAc = 20/1); ${ }^{1} \mathrm{H}$ NMR $\left(400 \mathrm{MHz}, \mathrm{CDCl}_{3}\right): \delta$ 7.34-7.27 (m, 3H), 7.23-7.09 (m, 5H), 7.08-7.03 (m, 2H), 6.97-6.92 (m, 1H), 6.80 (d, J = 8.8 Hz, 2H), 5.54 (s, 1H), 2.68-2.59 (m, 2H), 2.58-2.49 (m, 2H); ${ }^{13} \mathrm{C}$ NMR (100 MHz, $\left.\mathrm{CDCl}_{3}\right): \delta 157.5,152.0,137.9$, 131.6, 131.5, 130.1, 129.8, 128.4, 127.35, 127.28, 126.5, 126.3, 123.7, 122.2, 116.5, 113.2, 89.5, 84.1, 28.9, 26.0; HRMS (ESI) m/z: $[\mathrm{M}+\mathrm{Na}]^{+}$Calcd for $\mathrm{C}_{25} \mathrm{H}_{18} \mathrm{BrNNaO}_{2}$ 466.0413; Found 466.0421.

\section{(4R,9S)-2-(4-iodophenyl)-3-phenyl-9H-4,9-epoxy-3a,9a-ethanofuro[3,2-b]quinoline (4l)}

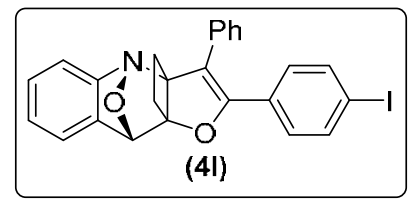

According to general procedure (GP1) with t-BuXPhosAuCl (2.6 mg, 4.0 $\mu \mathrm{mol}, 2 \mathrm{~mol} \%), \mathrm{AgSbF}_{6}$ (10.8 mg, $\left.31.4 \mu \mathrm{mol}, 15 \mathrm{~mol} \%\right)$, benzo[c]isoxazole $\quad 2 a \quad(60 \mu \mathrm{L}, \quad 0.60 \quad \mathrm{mmol}, \quad 3.0 \quad$ equiv $)$, 3-cyclopropylidene-1-(4-iodophenyl)-2-phenylprop-2-en-1-one 1e (73.3 mg, 0.197 mmol, 1.0 equiv), $100 \mathrm{mg} 4 \AA$ MS, $2.0 \mathrm{~mL}$ of dry DCE for 12 hours reaction. Purification by neutral silica gel column chromatography provided $4 \mathbf{l}$ as a white solid (72.9 mg, 75\%); M.p. 133-135 ${ }^{\circ} \mathrm{C}$ (Petroleum ether/EtOAc); $\mathrm{R}_{f}=0.15$ (Petroleum ether/EtOAc $\left.=20 / 1\right) ;{ }^{1} \mathrm{H}$ NMR $\left(400 \mathrm{MHz}, \mathrm{CDCl}_{3}\right): \delta 7.51(\mathrm{~d}, J=$ 8.0 Hz, 2H), 7.34-7.27 (m, 1H), 7.23-7.01 (m, 7H), 6.98-6.91 (m, 1H), 6.67 (d, $J=8.4$ Hz, 2H), 5.54 (s, 1H), 2.68-2.59 (m, 2H), 2.58-2.49 (m, 2H); ${ }^{13} \mathrm{C}$ NMR (100 MHz, $\left.\mathrm{CDCl}_{3}\right): \delta$ 157.6, 152.0, 137.9, 137.5, 131.6, 130.7, 129.8, 128.4, 127.4, 127.3, 126.5, 126.3, 122.2, 116.5, 113.3, 95.7, 89.5, 84.1, 28.9, 26.0; HRMS (ESI) m/z: [M + H] Calcd for $\mathrm{C}_{25} \mathrm{H}_{19} \mathrm{INO}_{2}$ 492.0455; Found 492.0461.

(4R,9S)-2-(2-fluorophenyl)-3-phenyl-9H-4,9-epoxy-3a,9a-ethanofuro[3,2-b]quinoline (4m)

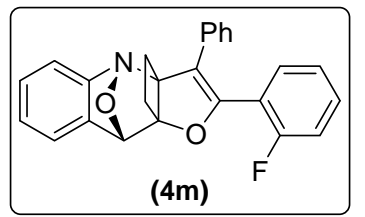

According to general procedure (GP1) with $t$-BuXPhosAuCl $(2.7 \mathrm{mg}, 4.1$ 
$\mu \mathrm{mol}, 2 \mathrm{~mol} \%), \operatorname{AgSbF}_{6}(11.0 \mathrm{mg}, 32.0 \mu \mathrm{mol}, 15 \mathrm{~mol} \%)$, benzo[c]isoxazole 2a (60 $\mu \mathrm{L}, 0.60 \mathrm{mmol}$, 3.0 equiv), 3-cyclopropylidene-1-(2-fluorophenyl)-2-phenylprop-2-en-1-one 1 f (52.7 mg, 0.200 mmol, 1.0 equiv), $100 \mathrm{mg} 4 \AA \mathrm{MS}, 2.0 \mathrm{~mL}$ of dry DCE for 19 hours reaction. Purification by flash column chromatography provided $\mathbf{4 m}$ as a white solid (51.5 mg, 67\%); M.p. 148-150 ${ }^{\circ} \mathrm{C}$ (Petroleum ether/EtOAc); $\mathrm{R}_{f}=0.17$ (Petroleum ether/EtOAc $\left.=20 / 1\right) ;{ }^{1} \mathrm{H}$ NMR $\left(400 \mathrm{MHz}, \mathrm{CDCl}_{3}\right): \delta$ 7.35-7.26 (m, 2H), 7.22-7.03 (m, 7H), 7.02-6.90 (m, 3H), 6.53 (t, $J=7.2$ Hz, 1H), 5.54 (s, 1H), 2.70-2.50 (m, 4H); ${ }^{13} \mathrm{C}$ NMR (100 MHz, $\left.\mathrm{CDCl}_{3}\right): \delta 159.9(\mathrm{~d}, J=251.1 \mathrm{~Hz}), 153.5,152.0,137.9,131.5$ (d, $J=7.7$ Hz), 131.3, 130.6, 128.3, 127.4, 126.5, 126.3 (d, $J=16.6 \mathrm{~Hz}), 124.1,122.3,119.4$ (d, $J=15.1 \mathrm{~Hz}$ ), 116.5, 116.1 (d, $J=20.7 \mathrm{~Hz}), 115.3,90.1$, 84.0, 83.6, 29.0, 26.0; HRMS (ESI) m/z: [M + H] Calcd for $\mathrm{C}_{25} \mathrm{H}_{19} \mathrm{FNO}_{2}$ 384.1394; Found 384.1398.

(4R,9S)-2-(2-bromophenyl)-3-phenyl-9H-4,9-epoxy-3a,9a-ethanofuro[3,2-b]quinoline (4n)

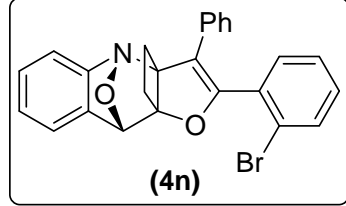

$(4 n)$ According to general procedure (GP1) with $t$-BuXPhosAuCl (2.7 mg, 4.1 $\mu \mathrm{mol}, 2 \mathrm{~mol} \%), \operatorname{AgSbF}_{6}(11.1 \mathrm{mg}, 32.3 \mu \mathrm{mol}, 15 \mathrm{~mol} \%)$, benzo[c]isoxazole 2a $\quad(60 \quad \mu \mathrm{L}, \quad 0.60 \quad$ mmol, $\quad 3.0 \quad$ equiv $)$, 1-(2-bromophenyl)-3-cyclopropylidene-2-phenylprop-2-en-1-one $1 \mathrm{~g}$ (65.3 mg, $0.201 \mathrm{mmol}, 1.0$ equiv), $100 \mathrm{mg} 4 \AA \mathrm{MS}, 2.0 \mathrm{~mL}$ of dry DCE for 19 hours reaction. Purification by flash column chromatography provided 4n as a white solid (48.1 mg, 54\%); M.p. 150-151 ${ }^{\circ} \mathrm{C}$ (Petroleum ether/EtOAc); $\mathrm{R}_{f}=0.20$ (Petroleum ether/EtOAc $\left.=20 / 1\right) ;{ }^{1} \mathrm{H}$ NMR $\left(400 \mathrm{MHz}, \mathrm{CDCl}_{3}\right): \delta 7.56(\mathrm{~d}, J=$ 8.0 Hz, 1H), 7.34 (d, $J=6.8 \mathrm{~Hz}, 1 \mathrm{H}), 7.22-7.05$ (m, 7H), 6.99 (t, $J=6.8 \mathrm{~Hz}, 3 \mathrm{H}), 6.01$ (t, $J=7.2 \mathrm{~Hz}$, 1H), 5.55 (s, 1H), 2.75-2.63 (m, 3H), 2.60-2.50 (m, 1H); ${ }^{13} \mathrm{C}$ NMR (100 MHz, $\left.\mathrm{CDCl}_{3}\right): \delta 157.8$, 152.2, 138.3, 133.0, 132.8, 131.0, 130.9, 130.6, 128.4, 127.6, 127.4, 126.3, 126.20, 126.17, 123.5, 122.5, 116.6, 114.6, 90.7, 84.0, 83.5, 29.1, 25.7; HRMS (ESI) m/z: $[\mathrm{M}+\mathrm{H}]^{+}$Calcd for $\mathrm{C}_{25} \mathrm{H}_{19} \mathrm{BrNO}_{2}$ 444.0594; Found 444.0598.

(4R,9S)-3-phenyl-2-(p-tolyl)-9H-4,9-epoxy-3a,9a-ethanofuro[3,2-b]quinoline (4o)

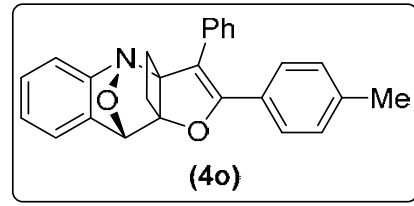

3-cyclopropylidene-2-phenyl-1-(p-tolyl)prop-2-en-1-one $\mathbf{1 h}$ (51.9 mg, 0.200 mmol, 1.0 equiv), 100 mg $4 \AA$ MS, $2.0 \mathrm{~mL}$ of dry DCE for 30 minutes reaction. Purification by flash column chromatography provided 40 as a white solid (52.3 mg, 69\%); M.p. 130-132 ${ }^{\circ} \mathrm{C}$ (Petroleum 
ether/EtOAc); $\mathrm{R}_{f}=0.21$ (Petroleum ether/EtOAc $\left.=20 / 1\right) ;{ }^{1} \mathrm{H}$ NMR (400 MHz, acetone- $\left.\mathrm{d}_{6}\right): \delta$ 7.37-7.32 (m, 1H), 7.22-7.16 (m, 2H), 7.15-7.06 (m, 5H), 7.05-7.01 (m, 2H), 6.93-6.90 (m, 1H), 6.85-6.76 (m, 2H), 5.68 (s, 1H), 2.55-2.46 (m, 4H), 2.27 (s, 3H); ${ }^{13} \mathrm{C}$ NMR (100 MHz, acetone- $\left.\mathrm{d}_{6}\right): \delta$ 160.2, 153.4, 140.4, 139.7, 133.3, 129.6, 129.5, 129.01, 128.96, 128.0, 127.8, 126.9, 126.8, 123.2, 117.0, 113.0, 90.3, 84.7, 84.5, 26.5, 21.3, 21.3; HRMS (ESI) m/z: $[\mathrm{M}+\mathrm{H}]^{+}$Calcd for $\mathrm{C}_{26} \mathrm{H}_{22} \mathrm{NO}_{2}$ 380.1645; Found 380.1651.

\section{(4R,9S)-2-(4-methoxyphenyl)-3-phenyl-9H-4,9-epoxy-3a,9a-ethanofuro[3,2-b]quinoline (4p)}

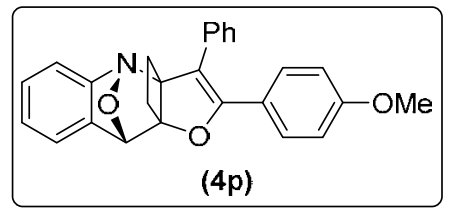

According to general procedure (GP1) with t-BuXPhosAuCl (2.5 mg, $3.9 \mu \mathrm{mol}, 2 \mathrm{~mol} \%), \operatorname{AgSbF}_{6}(10.7 \mathrm{mg}, 31.1 \mu \mathrm{mol}, 15 \mathrm{~mol} \%)$, benzo[c]isoxazole $\quad 2 a \quad(60 \mu \mathrm{L}, \quad 0.60 \quad$ mmol, $3.0 \quad$ equiv $)$, 3-cyclopropylidene-1-(4-methoxyphenyl)-2-phenylprop-2-en-1-one 1i (55.0 mg, 0.199 mmol, 1.0 equiv), $100 \mathrm{mg} 4 \AA \mathrm{MS}, 2.0 \mathrm{~mL}$ of dry DCE for 1 hour reaction. Purification by flash column chromatography provided 4p as a white solid (59.9 mg, 76\%); M.p. 142-143 ${ }^{\circ} \mathrm{C}$ (Petroleum ether/EtOAc); $\mathrm{R}_{f}=0.10$ (Petroleum ether/EtOAc $=20 / 1$ ); ${ }^{1} \mathrm{H}$ NMR (400 MHz, acetone- $\mathrm{d}_{6}$ ): $\delta$ 7.37-7.31 (m, 1H), 7.23-7.02 (m, 7H), 6.94 (d, $J=7.2 \mathrm{~Hz}, 1 \mathrm{H}), 6.87-6.83$ (m, 2H), 6.80-6.74 (m, 2H), 5.68 (s, 1H), 3.76 (s, 3H), 2.55-2.46 (m, 4H); ${ }^{13} \mathrm{C}$ NMR (100 MHz, acetone- $\left.\mathrm{d}_{6}\right): \delta$ 161.5, 160.6, 153.5, 139.7, 133.4, 130.5, 129.0, 128.0, 127.8, 126.8, 124.5, 123.2, 117.0, 114.4, 112.4, 90.2, 84.7, 84.5, 55.6, 26.5; HRMS (ESI) m/z: [M + H $]^{+}$Calcd for $\mathrm{C}_{26} \mathrm{H}_{22} \mathrm{NO}_{3}$ 396.1594; Found 396.1595.

\section{(4R,9S)-2-(naphthalen-2-yl)-3-phenyl-9H-4,9-epoxy-3a,9a-ethanofuro[3,2-b]quinoline (4q)}

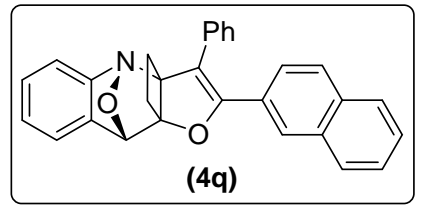

According to general procedure (GP1) with $t$-BuXPhosAuCl (2.9 mg, 4.4 $\mu \mathrm{mol}, 2 \mathrm{~mol} \%), \mathrm{AgSbF}_{6}$ (11.1 mg, $\left.32.3 \mu \mathrm{mol}, 15 \mathrm{~mol} \%\right)$, benzo[c]isoxazole $2 a \quad(60 \mu \mathrm{L}, \quad 0.60 \quad \mathrm{mmol}, \quad 3.0 \quad$ equiv $)$, 3-cyclopropylidene-1-(naphthalen-2-yl)-2-phenylprop-2-en-1-one 1j (59.8 mg, 0.202 mmol, 1.0 equiv), $100 \mathrm{mg} 4 \AA \mathrm{MS}, 2.0 \mathrm{~mL}$ of dry DCE for 3 hours reaction. Purification by flash column chromatography provided 4q as a white solid (48.2 mg, 57\%); M.p. 149-150 ${ }^{\circ} \mathrm{C}$ (Petroleum ether/EtOAc); $\mathrm{R}_{f}=0.21$ (Petroleum ether/EtOAc = 20/1); ${ }^{1} \mathrm{H}$ NMR (400 MHz, acetone- $\left.\mathrm{d}_{6}\right): \delta 7.85(\mathrm{~d}$, $J=7.6 \mathrm{~Hz}, 1 \mathrm{H}), 7.80-7.75$ (m, 1H), 7.72 (d, $J=8.8 \mathrm{~Hz}, 1 \mathrm{H}), 7.57-7.46$ (m, 3H), 7.36 (d, $J=7.2 \mathrm{~Hz}$, 1H), 7.22-7.12 (m, 6H), 7.11-7.05 (m, 1H), 6.99 (d, $J=7.6$ Hz, 1H), 6.94-6.89 (m, 1H), 5.74 (m, 1H), 2.65-2.50 (m, 4H); ${ }^{13} \mathrm{C}$ NMR (100 MHz, acetone- $\left.\mathrm{d}_{6}\right): \delta 159.8,153.5,139.7,134.6,133.8,133.1$, 130.9, 129.8, 129.1, 129.0, 128.8, 128.6, 128.5, 128.2, 127.95, 127.87, 127.3, 127.1, 126.9, 126.2, 
123.3, 117.1, 113.9, 90.684.8, 84.6, 26.5; HRMS (ESI) m/z: $[\mathrm{M}+\mathrm{H}]^{+}$Calcd for $\mathrm{C}_{29} \mathrm{H}_{22} \mathrm{NO}_{2}$ 416.1645; Found 416.1646.

(4R,9S)-3-phenyl-2-(thiophen-2-yl)-9H-4,9-epoxy-3a,9a-ethanofuro[3,2-b]quinoline (4r)

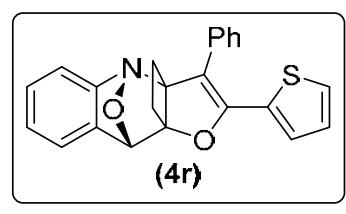

(4r)

According to general procedure (GP1) with $t$-BuXPhosAuCl $(2.7 \mathrm{mg}, 4.1$ $\mu \mathrm{mol}, 2 \mathrm{~mol} \%), \operatorname{AgSbF}_{6}(11.0 \mathrm{mg}, 32.0 \mu \mathrm{mol}, 15 \mathrm{~mol} \%)$, benzo[c]isoxazole 2a

$\mu \mathrm{L}, \quad 0.60$ mmol,

3.0 equiv),

3-cyclopropylidene-2-phenyl-1-(thiophen-2-yl)prop-2-en-1-one 11 (49.6 mg, 0.197 mmol, 1.0 equiv), $100 \mathrm{mg} 4 \AA \mathrm{MS}, 2.0 \mathrm{~mL}$ of dry DCE for 1 hour reaction. Purification by flash column chromatography provided 4r as a white solid (44.3 mg, 61\%); M.p. 150-152 ${ }^{\circ} \mathrm{C}$ (Petroleum ether/EtOAc); $\mathrm{R}_{f}=0.22($ Petroleum ether/EtOAc $=20 / 1) ;{ }^{1} \mathrm{H}$ NMR $\left(400 \mathrm{MHz}, \mathrm{CDCl}_{3}\right): \delta$ 7.36-7.12 (m, 7H), 7.10-7.01 (m, 2H), 6.98-6.90 (m, 1H), 6.85-6.78 (m, 2H), 5.53 (s, 1H), 2.68-2.49 (m, 4H); ${ }^{13} \mathrm{C}$ NMR (100 MHz, $\left.\mathrm{CDCl}_{3}\right): \delta 152.2,151.9,137.8,132.0,131.7,128.4,128.0,127.7,127.4,127.3$, 126.9, 126.8, 126.4, 122.3, 116.6, 113.1, 89.3, 84.4, 84.1, 29.0, 26.1; HRMS (ESI) m/z: [M + H] Calcd for $\mathrm{C}_{23} \mathrm{H}_{18} \mathrm{NO}_{2} \mathrm{~S} 372.1053$; Found 372.1056.

(4R,9S)-2-(furan-2-yl)-3-phenyl-9H-4,9-epoxy-3a,9a-ethanofuro[3,2-b]quinoline (4s)

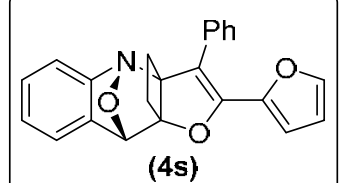

(4s)

According to general procedure (GP1) with $t$-BuXPhosAuCl (2.7 mg, 4.1 $\mu \mathrm{mol}, 2 \mathrm{~mol} \%), \operatorname{AgSbF}_{6}(11.0 \mathrm{mg}, 32.0 \mu \mathrm{mol}, 15 \mathrm{~mol} \%)$, benzo[c]isoxazole

2a

$(60$

$\mu \mathrm{L}$,

0.60

mmol,

3.0

equiv),

3-cyclopropylidene-1-(furan-2-yl)-2-phenylprop-2-en-1-one 1m (47.6 mg, 0.201 mmol, 1.0 equiv), $100 \mathrm{mg} 4 \AA \mathrm{MS}, 2.0 \mathrm{~mL}$ of dry DCE for 1 hour reaction. Purification by flash column chromatography provided $4 \mathbf{s}$ as a white solid (32.8 mg, 46\%); M.p. 110-112 ${ }^{\circ} \mathrm{C}$ (Petroleum ether/EtOAc); $\mathrm{R}_{f}=0.29$ (Petroleum ether/EtOAc = 20/1); ${ }^{1} \mathrm{H}$ NMR (400 MHz, acetone- $\mathrm{d}_{6}$ ): $\delta$ 7.45-7.41 (m, 1H), 7.38-7.27 (m, 5H), 7.26-7.18 (m, 1H), 7.06-7.02 (m, 2H), 6.93-6.86 (m, 1H), 6.42-6.38 (m, 1H), 6.29 (d, $J=3.6 \mathrm{~Hz}, 1 \mathrm{H}), 5.69$ (s, 1H), 2.57-2.45 (m, 4H); ${ }^{13} \mathrm{C}$ NMR (100 MHz, acetone- $\left.\mathrm{d}_{6}\right): \delta 153.2,149.4,145.9,144.3,144.2,139.5,132.6,128.9,128.7,127.8,127.5,126.8$, 123.1, 117.2, 114.0, 112.5, 111.9, 90.5, 84.9, 84.5, 26.5; HRMS (ESI) m/z: $[\mathrm{M}+\mathrm{H}]^{+}$Calcd for $\mathrm{C}_{23} \mathrm{H}_{18} \mathrm{NO}_{3} 356.1281$; Found 356.1284.

\section{(4R,9S)-3-(3-fluorophenyl)-2-phenyl-9H-4,9-epoxy-3a,9a-ethanofuro[3,2-b]quinoline (4t)}

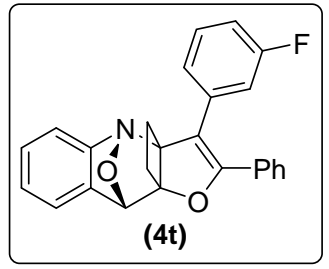

According to general procedure (GP1) with $t$-BuXPhosAuCl (2.8 mg, $4.3 \mu \mathrm{mol}$, 
$2 \mathrm{~mol} \%), \operatorname{AgSbF}_{6}(10.2 \mathrm{mg}, 29.7 \mu \mathrm{mol}, 15 \mathrm{~mol} \%$ ), benzo[c]isoxazole 2a (60 $\mu \mathrm{L}, 0.60 \mathrm{mmol}, 3.0$ equiv), 3-cyclopropylidene-2-(3-fluorophenyl)-1-phenylprop-2-en-1-one 1n (52.2 mg, 0.198 mmol, 1.0 equiv), $100 \mathrm{mg} 4 \AA \mathrm{MS}, 2.0 \mathrm{~mL}$ of dry DCE for 4 hours reaction. Purification by flash column chromatography provided 4t as a white solid (50.5 mg, 67\%); M.p. 154-156 ${ }^{\circ} \mathrm{C}$ (Petroleum ether/EtOAc); $\mathrm{R}_{f}=0.20$ (Petroleum ether/EtOAc = 20/1); ${ }^{1} \mathrm{H}$ NMR (400 MHz, acetone- $\mathrm{d}_{6}$ ): $\delta$ 7.41-7.32 (m, 2H), 7.31-7.20 (m, 3H), 7.19-7.08 (m, 2H), 6.99-6.86 (m, 5H), 6.75-6.67 (m, 1H), 5.74 (s, 1H), 2.61-2.48 (m, 4H); ${ }^{13} \mathrm{C}$ NMR (100 MHz, acetone- $\mathrm{d}_{6}$ ): $\delta 163.4$ (d, $\left.J=241.1 \mathrm{~Hz}\right), 161.4,153.3$, 139.5, 135.6 (d, $J=8.4 \mathrm{~Hz}$ ), 130.9 (d, $J=9.0 \mathrm{~Hz}$ ), 130.1 (d, $J=95.0 \mathrm{~Hz}$ ), 130.7, 129.3, 129.0, 128.5, 128.0, 127.5, 127.2, 127.0, 123.8 (d, $J=2.1 \mathrm{~Hz}$ ), 123.3, 116.9, 113.8 (d, $J=21.4 \mathrm{~Hz}$ ), 90.9, 84.5, 84.3, 26.4; HRMS (ESI) m/z: [M + H] Calcd for $\mathrm{C}_{25} \mathrm{H}_{19} \mathrm{FNO}_{2}$ 384.1394; Found 384.1400.

(4R,9S)-2-phenyl-3-(p-tolyl)-9H-4,9-epoxy-3a,9a-ethanofuro[3,2-b]quinoline (4u)

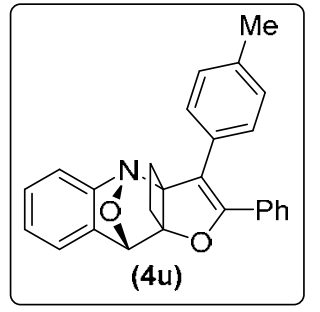

According to general procedure (GP1) with $t$-BuXPhosAuCl (2.6 mg, $4.0 \mu \mathrm{mol}$, $2 \mathrm{~mol} \%), \operatorname{AgSbF}_{6}(9.8 \mathrm{mg}, 28.5 \mu \mathrm{mol}, 15 \mathrm{~mol} \%$ ), benzo[c]isoxazole 2a (60 $\mu \mathrm{L}$, 0.60 mmol, 3.0 equiv), 3-cyclopropylidene-1-phenyl-2-( $p$-tolyl)prop-2-en-1-one 10 (51.8 mg, 0.199 mmol, 1.0 equiv), $100 \mathrm{mg} 4 \AA$ MS, $2.0 \mathrm{~mL}$ of dry DCE for 1 hour reaction. Purification by flash column chromatography provided $\mathbf{4 u}$ as a white solid (57.5 mg, 75\%); M.p. 130-132 ${ }^{\circ} \mathrm{C}$ (Petroleum ether/EtOAc); $\mathrm{R}_{f}=0.26$ (Petroleum ether/EtOAc $\left.=20 / 1\right) ;{ }^{1} \mathrm{H}$ NMR (400 MHz, acetone- $\mathrm{d}_{6}$ ): $\delta$ 7.38-7.27 (m, 2H), 7.26-7.19 (m, 2H), 7.15-7.05 (m, 2H), 7.00 (s, 4H), 6.97-6.88 (m, 3H), 5.69 (s, 1H), 2.55-2.45 (m, 4H), 2.25 (s, 3H); ${ }^{13} \mathrm{C}$ NMR (100 MHz, acetone- $\left.\mathrm{d}_{6}\right): \delta 159.3,153.4,139.7,136.6,132.5,130.2,130.1,129.7,129.0,128.0,127.8,126.8$, 123.2, 117.0, 113.3, 90.4, 84.7, 84.5, 26.4, 21.0; HRMS (ESI) m/z: $[\mathrm{M}+\mathrm{Na}]^{+}$Calcd for $\mathrm{C}_{26} \mathrm{H}_{21} \mathrm{NNaO}_{2}$ 402.1465; Found 402.1466.

\section{(4R,9S)-3-(4-(tert-butyl)phenyl)-2-phenyl-9H-4,9-epoxy-3a,9a-ethanofuro[3,2-b]quinoline (4v)}

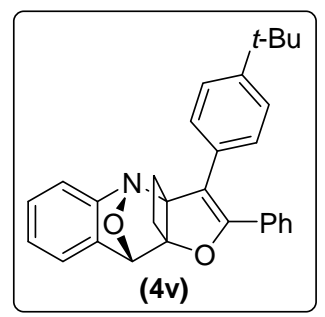

According to general procedure (GP1) with $t$-BuXPhosAuCl (2.7 mg, $4.1 \mu \mathrm{mol}$, $2 \mathrm{~mol} \%), \operatorname{AgSbF}_{6}(10.3 \mathrm{mg}, 29.9 \mu \mathrm{mol}, 15 \mathrm{~mol} \%)$, benzo[c]isoxazole 2a (60 $\mu \mathrm{L}, \quad 0.60 \quad$ mmol, $\quad 3.0 \quad$ equiv), 2-(4-(tert-butyl)phenyl)-3-cyclopropylidene-1-phenylprop-2-en-1-one 1p (60.3

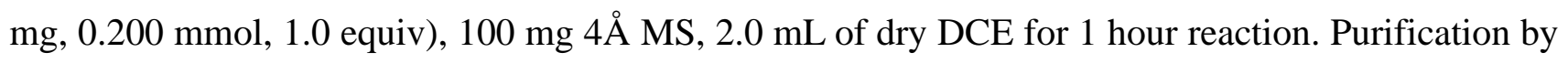
flash column chromatography provided $\mathbf{4 v}$ as a white solid (54.7 mg, 65\%); M.p. 137-139 ${ }^{\circ} \mathrm{C}$ (Petroleum ether/EtOAc); $\mathrm{R}_{f}=0.35$ (Petroleum ether/EtOAc $\left.=10 / 1\right) ;{ }^{1} \mathrm{H}$ NMR $(400 \mathrm{MHz}$, 
acetone- $\left.\mathrm{d}_{6}\right): \delta$ 7.38-7.28 (m, 2H), 7.27-7.20 (m, 4H), 7.20-7.03 (m, 4H), 6.97-6.88 (m, 3H), $5.69(\mathrm{~s}$, 1H), 2.55-2.46 (m, 4H), 1.27 (s, 9H); ${ }^{13} \mathrm{C}$ NMR (100 MHz, acetone-d $\left.\mathrm{d}_{6}\right): \delta$ 159.4, 153.4, 149.7, 139.7, 132.6, 130.2, 130.0, 129.1, 129.0, 127.8, 127.6, 126.8, 125.9, 123.2, 117.0, 113.3, 90.4, 84.7, 84.5, 34.9, 31.5, 26.4; HRMS (ESI) m/z: [M + H] Calcd for $\mathrm{C}_{29} \mathrm{H}_{28} \mathrm{NO}_{2}$ 422.2115; Found 422.2118.

\section{(4R,9S)-3-(4-methoxyphenyl)-2-phenyl-9H-4,9-epoxy-3a,9a-ethanofuro[3,2-b]quinoline (4w)}

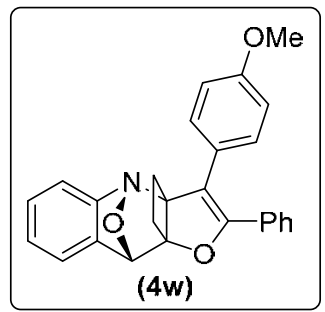

According to general procedure (GP1) with $t$-BuXPhosAuCl (2.6 mg, $4.0 \mu \mathrm{mol}$, $2 \mathrm{~mol} \%), \operatorname{AgSbF}_{6}(10.0 \mathrm{mg}, 29.1 \mu \mathrm{mol}, 15 \mathrm{~mol} \%)$, benzo[c]isoxazole 2a (60 $\begin{array}{llll}\mu \mathrm{L}, & 0.60 & \mathrm{mmol} & 3.0\end{array}$ 3-cyclopropylidene-2-(4-methoxyphenyl)-1-phenylprop-2-en-1-one $\quad \mathbf{1 q}$ (55.1 mg, $0.200 \mathrm{mmol}, 1.0$ equiv), $100 \mathrm{mg} 4 \AA \mathrm{MS}, 2.0 \mathrm{~mL}$ of dry DCE for 1 hour reaction. Purification by flash column chromatography provided $\mathbf{4 w}$ as a white solid (55.0 mg, 69\%); M.p. 151-153 ${ }^{\circ} \mathrm{C}$ (Petroleum ether/EtOAc); $\mathrm{R}_{f}=0.16$ (Petroleum ether/EtOAc $\left.=20 / 1\right) ;{ }^{1} \mathrm{H}$ NMR $(400 \mathrm{MHz}$, acetone- $\left.\mathrm{d}_{6}\right): \delta$ 7.38-7.33 (m, 1H), 7.31-7.27 (m, 1H), 7.26-7.19 (m, 2H), 7.15-7.05 (m, 2H), 7.06-7.00 (m, 2H), 6.97-6.88 (m, 3H), 5.80-6.73 (m, 2H), 5.68 (s, 1H), 3.76 (s, 3H), 2.57-2.46 (m, $4 \mathrm{H}) ;{ }^{13} \mathrm{C}$ NMR (100 MHz, acetone- $\left.\mathrm{d}_{6}\right): \delta$ 159.1, 158.4, 153.4, 139.7, 132.5, 130.1, 129.3, 129.2, 129.0, 127.8, 126.8, 125.2, 123.2, 117.0, 114.5, 113.0, 90.2, 84.8, 84.5, 55.4, 26.4; HRMS (ESI) m/z: $[\mathrm{M}+\mathrm{H}]^{+}$Calcd for $\mathrm{C}_{26} \mathrm{H}_{22} \mathrm{NO}_{3}$ 396.1594; Found 396.1593.

(4R,9S)-3-(naphthalen-1-yl)-2-phenyl-9H-4,9-epoxy-3a,9a-ethanofuro[3,2-b]quinoline (4x)

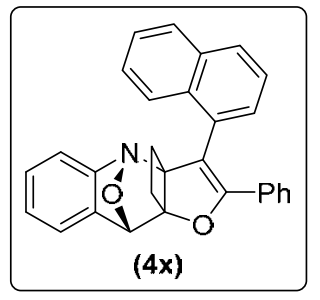

According to general procedure (GP1) with $t$-BuXPhosAuCl (2.9 mg, $4.4 \mu \mathrm{mol}$, $2 \mathrm{~mol} \%), \operatorname{AgSbF}_{6}(10.2 \mathrm{mg}, 29.7 \mu \mathrm{mol}, 15 \mathrm{~mol} \%)$, benzo[c]isoxazole $2 \mathbf{a}(60$ $\begin{array}{llll}\mu \mathrm{L}, & 0.60 & \mathrm{mmol} & 3.0\end{array}$ 3-cyclopropylidene-2-(naphthalen-1-yl)-1-phenylprop-2-en-1-one 1r (59.4 mg, $0.200 \mathrm{mmol}, 1.0$ equiv), $100 \mathrm{mg} 4 \AA \mathrm{MS}, 2.0 \mathrm{~mL}$ of dry DCE for 2 hours reaction. Purification by flash column chromatography provided $\mathbf{4 x}$ as a white solid (27.0 $\mathrm{mg}$, 32\%, unstable when take NMR); M.p. 98-100 ${ }^{\circ} \mathrm{C}$ (Petroleum ether/EtOAc); $\mathrm{R}_{f}=0.18$ (Petroleum ether/EtOAc $=20 / 1$ ); ${ }^{1} \mathrm{H}$ NMR (400 MHz, acetone- $\left.\mathrm{d}_{6}\right): \delta$ 7.89-7.80 (m, 3H), 7.61 (t, $\left.J=7.0 \mathrm{~Hz}, 1 \mathrm{H}\right), 7.48-7.37$ (m, 2H), 7.36-7.24 (m, 2H), 7.15-7.00 (m, 4H), 6.95-6.87 (m, 2H), 6.85 (d, J = 7.6 Hz, 2H), 5.78 (s, 1H), 2.79-2.71 (m, 1H), 2.66-2.56 (m, 2H), 2.48-2.40 (m, 1H); ${ }^{13} \mathrm{C}$ NMR (100 MHz, acetone- $\left.\mathrm{d}_{6}\right): \delta 160.7$, 153.7, 140.5, 135.1, 131.83, 131.78, 131.6, 129.9, 129.6, 129.0, 128.8, 128.5, 127.95, 127.91, 127.7, 127.1, 126.9, 126.4, 126.3, 126.1, 123.1, 118.3, 109.1, 90.5, 87.3, 84.8, 27.0; HRMS (ESI) m/z: [M + 
$\mathrm{H}]^{+}$Calcd for $\mathrm{C}_{29} \mathrm{H}_{22} \mathrm{NO}_{2}$ 416.1645; Found 416.1649.

(4R,9S)-3-(2,5-dimethoxyphenyl)-2-phenyl-9H-4,9-epoxy-3a,9a-ethanofuro[3,2-b]quinoline (4y)

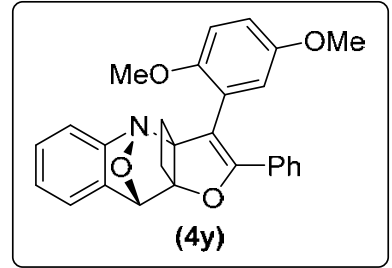

According to general procedure (GP1) with $t$-BuXPhosAuCl (2.8 mg, 4.1 $\mu \mathrm{mol}, 2 \mathrm{~mol} \%), \mathrm{AgSbF}_{6}(10.3 \mathrm{mg}, 29.9 \mu \mathrm{mol}, 15 \mathrm{~mol} \%)$, benzo[c]isoxazole $\quad$ 2a $\quad(60 \quad \mu \mathrm{L}, \quad 0.60 \quad \mathrm{mmol}, \quad 3.0 \quad$ equiv $)$, 3-cyclopropylidene-2-(2,5-dimethoxyphenyl)-1-phenylprop-2-en-1-one $\quad \mathbf{1 s}$

(61.8 mg, $0.202 \mathrm{mmol}, 1.0$ equiv), $100 \mathrm{mg} 4 \AA \mathrm{MS}, 2.0 \mathrm{~mL}$ of dry DCE for 3.5 hours reaction. Purification by flash column chromatography provided $\mathbf{4 y}$ as a white solid (65.3 $\mathrm{mg}, 76 \%$, unstable when take NMR); M.p. $122-124{ }^{\circ} \mathrm{C}\left(\right.$ Petroleum ether/EtOAc); $\mathrm{R}_{f}=0.14$ (Petroleum ether $/$ EtOAc $=$ 20/1); ${ }^{1} \mathrm{H}$ NMR (400 MHz, acetone- $\mathrm{d}_{6}$ ): $\delta$ 7.46-7.41 (m, 1H), 7.22-7.15 (m, 1H), 7.14-7.06 (m, 4H), 6.92-6.80 (m, 4H), 6.78-6.72 (m, 1H), 6.38 (d, J = 3.2 Hz, 1H), 5.66 (s, 1H), 3.55 (s, 3H), 3.45 (s, 3H), 2.86-2.79 (m, 1H), 2.52-2.43 (m, 3H); ${ }^{13} \mathrm{C}$ NMR (100 MHz, acetone- $\left.\mathrm{d}_{6}\right): \delta 161.3,153.9,153.6$, 152.3, 139.9, 133.1, 129.6, 128.4, 128.1, 127.5, 126.8, 123.3, 122.8, 117.8, 117.5, 114.0, 113.1, 109.6, 90.4, 85.5, 84.5, 55.6, 30.8, 26.8; HRMS (ESI) m/z: $[\mathrm{M}+\mathrm{H}]^{+}$Calcd for $\mathrm{C}_{27} \mathrm{H}_{24} \mathrm{NO}_{4} 426.1700$; Found 426.1707.

(4R,9S)-2-(4-chlorophenyl)-3-(2-methoxyphenyl)-9H-4,9-epoxy-3a,9a-ethanofuro[3,2-b]quinoline (4z)

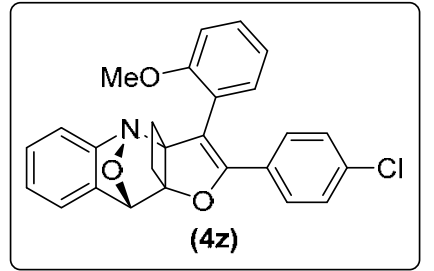

According to general procedure (GP1) with $t$-BuXPhosAuCl (2.8 mg, 4.1 $\mu \mathrm{mol}, 2 \mathrm{~mol} \%)$, AgSbF$_{6}$ (9.7 mg, $\left.28.2 \mu \mathrm{mol}, 15 \mathrm{~mol} \%\right)$, benzo[c]isoxazole $\quad 2 a \quad(60 \mu \mathrm{L}, \quad 0.60 \quad \mathrm{mmol}, \quad 3.0 \quad$ equiv $)$, 1-(4-chlorophenyl)-3-cyclopropylidene-2-(2-methoxyphenyl)prop-2-en-1one 1t (63.5 mg, $0.204 \mathrm{mmol}, 1.0$ equiv), $100 \mathrm{mg} 4 \AA \mathrm{MS}, 2.0 \mathrm{~mL}$ of dry DCE for 2.5 hours reaction. Purification by flash column chromatography provided $\mathbf{4 z}$ as a white solid (65.3 mg, 74\%); M.p. $127-128{ }^{\circ} \mathrm{C}$ (Petroleum ether/EtOAc); $\mathrm{R}_{f}=0.18$ (Petroleum ether/EtOAc $\left.=20 / 1\right) ;{ }^{1} \mathrm{H}$ NMR $(400$ MHz, acetone- $\left.\mathrm{d}_{6}\right): \delta$ 7.43-7.38 (m, $\left.1 \mathrm{H}\right), 7.26-7.20(\mathrm{~m}, 1 \mathrm{H}), 7.17-7.12(\mathrm{~m}, 2 \mathrm{H}), 7.10-7.04(\mathrm{~m}, 2 \mathrm{H})$, 6.97-6.80 (m, 6H), 5.57 (s, 1H), 3.47 (s, 3H), 2.80-2.72 (m, 1H), 2.52-2.43 (m, 3H); ${ }^{13} \mathrm{C}$ NMR (100 MHz, acetone- $\left.\mathrm{d}_{6}\right): \delta 159.5,157.8,153.5,139.8,134.7,132.0,131.9,129.4,129.3,128.5,127.6$, 126.8, 122.8, 122.1 ,120.9, 117.8, 112.1, 110.0, 90.4, 85.7, 84.5, 54.93, 54.92, 30.6, 26.7; HRMS (ESI) $\mathrm{m} / \mathrm{z}:[\mathrm{M}+\mathrm{H}]^{+}$Calcd for $\mathrm{C}_{26} \mathrm{H}_{21} \mathrm{ClNO}_{3}$ 430.1204; Found 430.1207.

(5R,5bR,11S)-14,15-diphenyl-5b,7,8,9,10,10a-hexahydro-6H,11H-5,11-epoxy-10b,5a-(epoxyetheno 


\section{)cyclohepta[3,4]cyclobuta[1,2-b]quinoline (4aa)}

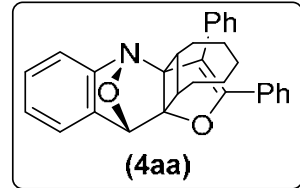

According to general procedure (GP1) with $t$-BuXPhosAuCl (1.4 mg, $2.1 \mu \mathrm{mol}$, $2 \mathrm{~mol} \%), \operatorname{AgSbF}_{6}(5.0 \mathrm{mg}, 15 \mu \mathrm{mol}, 15 \mathrm{~mol} \%)$, benzo[c]isoxazole 2a (30 $\mu \mathrm{L}$, 0.30 mmol, 3.0 equiv), 3-cycloheptylidene-1,2-diphenylprop-2-en-1-one $\mathbf{1 x}$ (32.1

mg, $0.102 \mathrm{mmol}, 1.0$ equiv), $50 \mathrm{mg} 4 \AA \mathrm{MS}, 1.0 \mathrm{~mL}$ of dry DCE for 1.5 hours reaction. After completion, reaction mixture washed with saturated $\mathrm{NaHCO}_{3}$ solution, concentrated under reduced pressure and purified by neutral silica gel column chromatography to provided 4aa as a yellow solid (23.1 mg, 50\%); M.p. 130-132 ${ }^{\circ} \mathrm{C}$ (Petroleum ether/EtOAc); $\mathrm{R}_{f}=0.60$ (Petroleum ether $/$ EtOAc $=$ 20/1); ${ }^{1} \mathrm{H}$ NMR (400 MHz, $\mathrm{CDCl}_{3}$ ): $\delta$ 7.35-7.26 (m, 2H), 7.22-7.14 (m, 4H), 7.14-7.00 (m, 6H), 6.86 (d, $J=7.6 \mathrm{~Hz}, 2 \mathrm{H}), 5.46$ (s, 1H), 3.02-2.93 (m, 1H), 2.81-2.72 (m, 1H), 2.06-1.93 (m, 2H), 1.84-1.57 (m, 4H), 1.40-1.31 (m, 1H), 1.22-1.00 (m, 3H); ${ }^{13} \mathrm{C}$ NMR (100 MHz, $\left.\mathrm{CDCl}_{3}\right): \delta 159.7,152.5,138.2$, 133.2, 131.5, 129.5, 128.33, 128.25, 127.9, 127.2, 127.1, 126.1, 125.8, 122.3, 116.4, 112.3, 87.9, 85.7, 84.0, 45.3, 42.7, 31.6, 29.8, 28.9, 27.9, 26.5; HRMS (ESI) m/z: [M + H] Calcd for $\mathrm{C}_{30} \mathrm{H}_{28} \mathrm{NO}_{2}$ 434.2115; Found 434.2120.

\section{Procedure and experiment data for compound 5}

(a) General procedure (GP2-A):

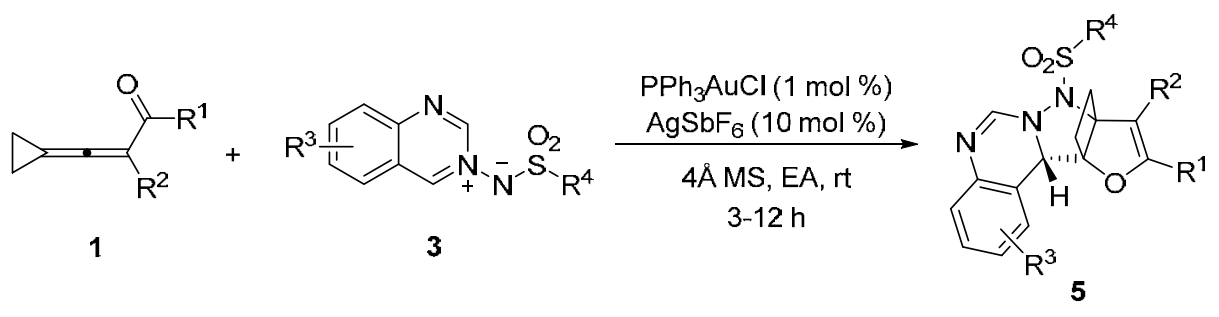

$\mathrm{PPh}_{3} \mathrm{AuCl}(2.0 \mu \mathrm{mol}, 1 \mathrm{~mol} \%)$ and $\mathrm{AgSbF}_{6}(20.0 \mu \mathrm{mol}, 10 \mathrm{~mol} \%)$ were dissolved in $2.0 \mathrm{~mL}$ of dry EA or THF in a dried vial under $\mathrm{N}_{2}$ atmosphere. Then, $4 \AA$ MS (100 mg) and $N$-iminoquinazolinium ylides 3 (0.22 mmol, 1.1 equiv) were added in this order and the reaction was stirred at room temperature. After being stirred for $5 \mathrm{~min}$, the 3-cyclopropylideneprop-2-en-1-ones 1 (0.20 mmol, 1.0 equiv) was added. The reaction was determined by TLC (3-12 h). After completion, the solution was removed under reduced pressure, and purified by flash silica gel chromatography to afford product 5.

(b) General procedure (GP2-B): 


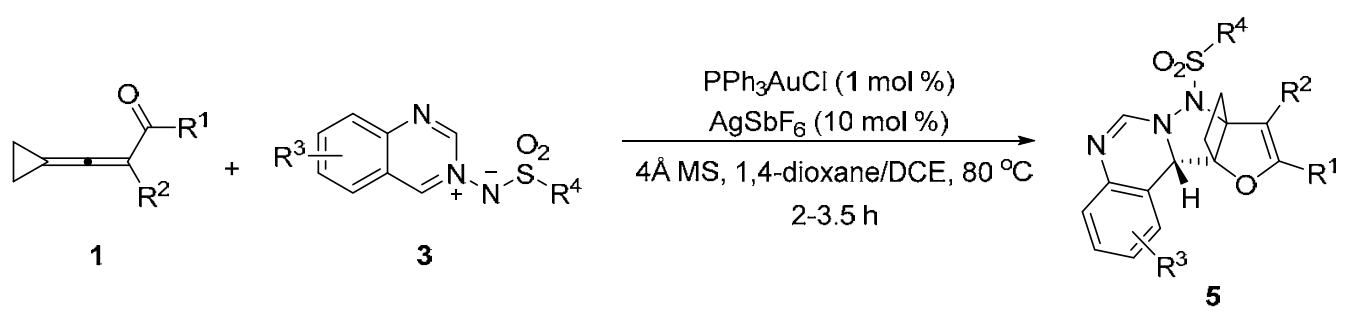

$\mathrm{PPh}_{3} \mathrm{AuCl}(2.0 \mu \mathrm{mol}, 1 \mathrm{~mol} \%)$ and $\operatorname{AgSbF}_{6}(20.0 \mu \mathrm{mol}, 10 \mathrm{~mol} \%)$ were dissolved in $2.0 \mathrm{~mL}$ of dry 1,4-dioxane and $2.0 \mathrm{~mL}$ of dry DCE in a dried vial under $\mathrm{N}_{2}$ atmosphere. Then, $4 \AA$ MS (100 mg) and $N$-iminoquinazolinium ylides 3 (0.22 mmol, 1.1 equiv) were added in this order and the reaction was stirred at $80{ }^{\circ} \mathrm{C}$ in an oil bath. After being stirred for $5 \mathrm{~min}$, the 3-Cyclopropylideneprop-2-en-1-ones 1 (0.20 mmol, 1.0 equiv) was added. The reaction was determined by TLC (2-3.5 h). After completion, the solution was removed under reduced pressure, and purified by flash silica gel chromatography to afford product 5.

(c) Experiment data:

(11aS,11bS)-9,10-diphenyl-8-tosyl-8H,11bH-8a,11a-ethanofuro[3',2':3,4]pyrazolo[1,5-c]quinazoli ne (5a)

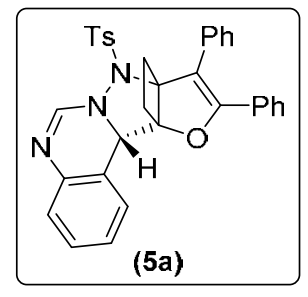

According to general procedure (GP2-A) with $\mathrm{PPh}_{3} \mathrm{AuCl}(0.9 \mathrm{mg}, 1.8 \mu \mathrm{mol}, 1$ mol \%), $\operatorname{AgSbF}_{6}(7 \mathrm{mg}, 21 \mu \mathrm{mol}, 10 \mathrm{~mol} \%$ ), quinazolin-3-ium-3-yl(tosyl)amide 3a $\quad(65.3 \quad \mathrm{mg}, \quad 0.218 \quad \mathrm{mmol}, 1.1 \quad$ equiv $)$, 3-cyclopropylidene-1,2-diphenylprop-2-en-1-one 1a (48.8 mg, $0.198 \mathrm{mmol}, 1.0$ equiv), $100 \mathrm{mg} 4 \AA \mathrm{MS}, 2.0 \mathrm{~mL}$ of dry EA for 4 hours reaction. Purification by flash column chromatography provided 5a as a white solid (87.5 mg, 82\%); $d r=9: 1$ (ratio was determined by ${ }^{1} \mathrm{H}$ NMR analysis of the crude product); when the reaction was carried out using method GP1, 5a was obtained in 50\% yield with 4.3:1 dr. M.p. $115-117{ }^{\circ} \mathrm{C}$ (Petroleum ether/EtOAc); $\mathrm{R}_{f}=0.26$ (Petroleum ether/EtOAc = 3/1); ${ }^{1} \mathrm{H}$ NMR $\left(400 \mathrm{MHz}_{\mathrm{CDCl}}\right): \delta 7.56(\mathrm{~d}, J=8.0 \mathrm{~Hz}, 2 \mathrm{H}), 7.43(\mathrm{~d}, J=$ 7.2 Hz, 2H), 7.36-7.25 (m, 4H), 7.24-7.05 (m, 10H), 6.42 (s, 1H), 5.16 (s, 1H), 3.40-3.22 (m, 1H), 3.10-2.97 (m, 1H), 2.74-2.65 (m, 1H), 2.52-2.40 (m, 1H), 2.32 (s, 3H); ${ }^{13} \mathrm{C} \mathrm{NMR} \mathrm{(100} \mathrm{MHz,} \mathrm{CDCl} 3$ ): $\delta$ 157.2, 150.7, 144.7, 139.5, 134.7, 132.6, 130.2, 129.5, 129.3, 129.2, 128.8, 128.4, 128.3, 128.0, 127.7, 127.1, 126.2, 126.12, 126.09, 120.4, 111.4, 94.4, 80.7, 65.6, 25.5, 25.3, 21.4; HRMS (ESI) $\mathrm{m} / \mathrm{z}:[\mathrm{M}+\mathrm{H}]^{+}$Calcd for $\mathrm{C}_{33} \mathrm{H}_{28} \mathrm{~N}_{3} \mathrm{O}_{3} \mathrm{~S}$ 546.1846; Found 546.1862.

(11aS,11bS)-3-chloro-9,10-diphenyl-8-tosyl-8H,11bH-8a,11a-ethanofuro[3',2':3,4]pyrazolo[1,5-c]q uinazoline (5b) 


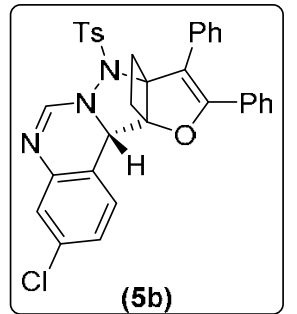

According to general procedure (GP2-B) with $\mathrm{PPh}_{3} \mathrm{AuCl}(1.1 \mathrm{mg}, 2.2 \mu \mathrm{mol}, 1$ mol \%), $\operatorname{AgSbF}_{6} \quad(7.2 \quad \mathrm{mg}, 21 \mu \mathrm{mol}, 10 \quad \mathrm{~mol} \%)$, (7-chloroquinazolin-3-ium-3-yl)(tosyl)amide $3 \mathbf{b}$ (73.2 mg, $0.220 \mathrm{mmol}, 1.1$ equiv), 3-cyclopropylidene-1,2-diphenylprop-2-en-1-one 1a (49.3 mg, 0.199 mmol, 1.0 equiv), $100 \mathrm{mg} 4 \AA \mathrm{MS}, 2.0 \mathrm{~mL}$ of dry 1,4-dioxane and $2.0 \mathrm{~mL}$ of dry DCE under $80{ }^{\circ} \mathrm{C}$ for 3.5 hours reaction. Purification by flash column chromatography provided $\mathbf{5 b}$ as a white solid (48.3 mg, 42\%); $d r=5$ : 1 (ratio was determined by ${ }^{1} \mathrm{H}$ NMR analysis of the crude product); M.p. 138-140 ${ }^{\circ} \mathrm{C}$ (Petroleum ether/EtOAc); $\mathrm{R}_{f}=0.47$ (Petroleum ether/EtOAc $\left.=3 / 1\right) ;{ }^{1} \mathrm{H}$ NMR $(400 \mathrm{MHz}$, $\left.\mathrm{CDCl}_{3}\right): \delta 7.56(\mathrm{~d}, J=7.6 \mathrm{~Hz}, 2 \mathrm{H}), 7.46-7.28$ (m, 5H), 7.27-7.06 (m, 10H), $6.41(\mathrm{~s}, 1 \mathrm{H}), 5.13(\mathrm{~s}, 1 \mathrm{H})$, 3.38-3.24 (m, 1H), 3.09-2.97 (m, 1H), 2.75-2.63 (m, 1H), 2.55-2.44 (m, 1H), 2.38 (s, 3H); ${ }^{13} \mathrm{C}$ NMR (100 MHz, $\left.\mathrm{CDCl}_{3}\right): \delta$ 157.3, 151.7, 145.0, 140.9, 134.70, 134.65, 132.5, 130.2, 129.7, 129.6, 128.8, 128.6, 128.4, 128.2, 127.8, 127.3, 127.2, 126.30, 126.25, 117.0, 111.5, 94.4, 80.8, 65.3, 25.6, 25.4, 21.6; HRMS (ESI) m/z: [M + H] Calcd for $\mathrm{C}_{33} \mathrm{H}_{27} \mathrm{ClN}_{3} \mathrm{O}_{3} \mathrm{~S}$ 580.1456; Found 580.1457.

(11aS,11bS)-3-bromo-9,10-diphenyl-8-tosyl-8H,11bH-8a,11a-ethanofuro[3',2':3,4]pyrazolo[1,5-c]q uinazoline (5c)

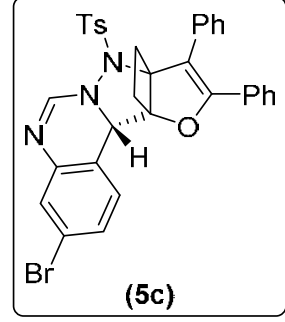

According to general procedure (GP2-B) with $\mathrm{PPh}_{3} \mathrm{AuCl}(1.1 \mathrm{mg}, 2.2 \mu \mathrm{mol}, 1$ mol \%), $\mathrm{AgSbF}_{6} \quad(6.8 \quad \mathrm{mg}, \quad 20 \mu \mathrm{mol}, 10 \quad \mathrm{~mol} \%)$, (7-bromoquinazolin-3-ium-3-yl)(tosyl)amide 3c (83.3 mg, 0.220 mmol, 1.1 equiv), 3-cyclopropylidene-1,2-diphenylprop-2-en-1-one 1a (49.8 mg, $0.202 \mathrm{mmol}, 1.0$ equiv), $100 \mathrm{mg} 4 \AA \mathrm{MS}, 2.0 \mathrm{~mL}$ of dry 1,4-dioxane and $2.0 \mathrm{~mL}$ of dry DCE under $80{ }^{\circ} \mathrm{C}$ for 3.5 hours reaction. Purification by flash column chromatography provided $5 \mathrm{c}$ as a white solid (77.2 mg, 61\%); $d r=5: 1$ (ratio was determined by ${ }^{1} \mathrm{H}$ NMR analysis of the crude product); M.p. 256-257 ${ }^{\circ} \mathrm{C}$ (Petroleum ether/EtOAc); $\mathrm{R}_{f}=0.53$ (Petroleum ether/EtOAc $\left.=3 / 1\right) ;{ }^{1} \mathrm{H}$ NMR $(400$ $\left.\mathrm{MHz}, \mathrm{CDCl}_{3}\right): \delta 7.56(\mathrm{~d}, J=8.0 \mathrm{~Hz}, 2 \mathrm{H}), 7.45-7.28(\mathrm{~m}, 7 \mathrm{H}), 7.26-7.14(\mathrm{~m}, 7 \mathrm{H}), 7.05(\mathrm{~d}, J=8.0 \mathrm{~Hz}$, 1H), 6.41 (s, 1H), 5.11 (s, 1H), 3.36-3.24 (m, 1H), 3.07-2.97 (m, 1H), 2.75-2.62 (m, 1H), 2.54-2.43 (m, 1H), 2.37 (s, 3H); ${ }^{13} \mathrm{C}$ NMR (100 MHz, $\left.\mathrm{CDCl}_{3}\right): \delta 157.3,151.7,144.9,141.1,134.6,132.5$, 130.2, 129.7, 129.5, 129.2, 129.1, 128.8, 128.5, 128.4, 128.2, 127.8, 127.5, 127.2, 122.6, 119.4, 111.4, 94.3, 80.7, 65.3, 25.5, 25.3, 21.6; HRMS (ESI) m/z: $[\mathrm{M}+\mathrm{H}]^{+}$Calcd for $\mathrm{C}_{33} \mathrm{H}_{27} \mathrm{BrN}_{3} \mathrm{O}_{3} \mathrm{~S}$ 624.0951; Found 624.0953.

\section{methyl}




\section{(11aS,11bS)-9,10-diphenyl-8-tosyl-8H,11bH-8a,11a-ethanofuro[3',2':3,4]pyrazolo[1,5-c]quinazoli}

\section{ne-3-carboxylate (5d)}

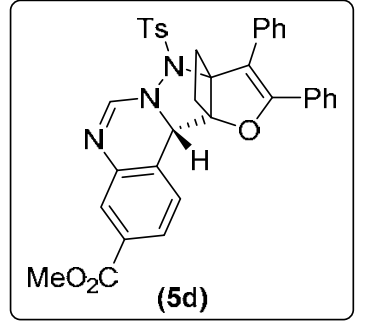

According to general procedure (GP2-B) with $\mathrm{PPh}_{3} \mathrm{AuCl}(1.2 \mathrm{mg}, 2.4 \mu \mathrm{mol}, 1$ mol \%), $\operatorname{AgSbF}_{6} \quad(7.3 \quad \mathrm{mg}, 21 \mu \mathrm{mol}, 10 \quad \mathrm{~mol} \%)$, (7-(methoxycarbonyl)quinazolin-3-ium-3-yl)(tosyl)amide 3d (78.7 mg, 0.220 mmol, 1.1 equiv), 3-cyclopropylidene-1,2-diphenylprop-2-en-1-one 1a (49.6 mg, $0.202 \mathrm{mmol}, 1.0$ equiv), $100 \mathrm{mg} 4 \AA \mathrm{MS}, 2.0 \mathrm{~mL}$ of dry 1,4-dioxane and $2.0 \mathrm{~mL}$ of dry DCE under $80{ }^{\circ} \mathrm{C}$ for 3.5 hours reaction. Purification by flash column chromatography provided 5d as a white solid (79.1 mg, 65\%); $d r=6: 1$ (ratio was determined by ${ }^{1} \mathrm{H}$ NMR analysis of the crude product); M.p. 205-207 ${ }^{\circ} \mathrm{C}$ (Petroleum ether/EtOAc); $\mathrm{R}_{f}=0.32$ (Petroleum ether/EtOAc = 3/1); ${ }^{1} \mathrm{H}$ NMR (400 MHz, $\left.\mathrm{CDCl}_{3}\right): \delta 7.90-7.85$ (m, 2H), $7.56(\mathrm{~d}, J=8.0 \mathrm{~Hz}, 2 \mathrm{H}), 7.42(\mathrm{~d}, J=7.2$ Hz, 2H), 7.37-7.29 (m, 3H), 7.28-7.13 (m, 8H), 6.45 (s, 1H), 5.20 (s, 1H), 3.90 (s, 3H), 3.36-3.28 (m, 1H), 3.10-3.00 (m, 1H), 2.79-2.69 (m, 1H), 2.56-2.45 (m, 1H), 2.37 (s, 3H); ${ }^{13} \mathrm{C}$ NMR (100 MHz, $\left.\mathrm{CDCl}_{3}\right): \delta 166.4,157.2,151.3,144.9,139.8,134.6,132.5,131.3,130.1,129.6,129.5,128.8,128.5$, 128.4, 128.1, 127.7, 127.3, 127.2, 126.3, 125.4, 111.5, 94.7, 80.8, 65.5, 52.1, 25.6, 25.4, 21.5; HRMS (ESI) m/z: [M + H] $]^{+}$Calcd for $\mathrm{C}_{35} \mathrm{H}_{30} \mathrm{~N}_{3} \mathrm{O}_{5} \mathrm{~S}$ 604.1901; Found 604.1899.

\section{(11aS,11bS)-2-fluoro-9,10-diphenyl-8-tosyl-8H,11bH-8a,11a-ethanofuro[3',2':3,4]pyrazolo[1,5-c]q}

\section{uinazoline (5e)}

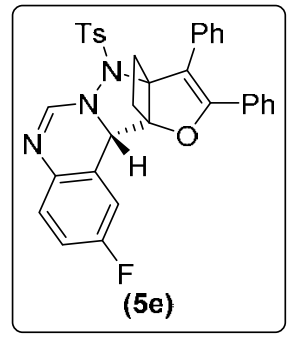

According to general procedure (GP2-A) with $\mathrm{PPh}_{3} \mathrm{AuCl}(0.9 \mathrm{mg}, 1.8 \mu \mathrm{mol}, 1$ mol \%), $\mathrm{AgSbF}_{6} \quad(7.1 \mathrm{mg}, 21 \mu \mathrm{mol}, 10 \mathrm{~mol} \%)$, (6-fluoroquinazolin-3-ium-3-yl)(tosyl)amide 3e (70.2 mg, 0.221 mmol, 1.1 equiv), 3-cyclopropylidene-1,2-diphenylprop-2-en-1-one 1a (49.3 mg, $0.200 \mathrm{mmol}, 1.0$ equiv), $100 \mathrm{mg} 4 \AA \mathrm{MS}, 2.0 \mathrm{~mL}$ of dry EA for 6 hours reaction. Purification by flash column chromatography provided 5e as a white solid (85.2 mg, 76\%); $d r=11$ : 1 (ratio was determined by ${ }^{1} \mathrm{H}$ NMR analysis of the crude product); M.p. 202-203 ${ }^{\circ} \mathrm{C}$ (Petroleum ether/EtOAc); $\mathrm{R}_{f}=0.39$ (Petroleum ether/EtOAc $\left.=3 / 1\right) ;{ }^{1} \mathrm{H}$ NMR (400 MHz, $\left.\mathrm{CDCl}_{3}\right): \delta 7.57(\mathrm{~d}, J=8.0 \mathrm{~Hz}, 2 \mathrm{H})$, 7.41 (d, $J=6.8 \mathrm{~Hz}, 2 \mathrm{H}), 7.37-7.26$ (m, 3H), 7.25-7.10 (m, 8H), 7.02-6.94 (m, 1H), 6.92-6.86 (m, 1H), 6.37 (s, 1H), 5.12 (s, 1H), 3.36-3.24 (m, 1H), 3.07-2.96 (m, 1H), 2.74-2.64 (m, 1H), 2.54-2.43 (m, 1H), 2.37 (s, 3H); ${ }^{13} \mathrm{C}$ NMR (100 MHz, $\left.\mathrm{CDCl}_{3}\right): \delta 160.5$ (d, $\left.J=244.8 \mathrm{~Hz}\right), 157.2,150.1,144.9$, 136.0 (d, $J=1.7 \mathrm{~Hz}), 134.8,132.6,130.3,129.6,129.5,128.9,128.6,128.4,128.2,127.9,127.8$, 
127.2, 122.2 (d, $J=8.2 \mathrm{~Hz}), 116.0$ (d, $J=22.0 \mathrm{~Hz}$ ), 113.1 (d, $J=23.7 \mathrm{~Hz}), 111.6,94.5$, 80.9, 65.6, 25.5, 25.4, 21.5; HRMS (ESI) m/z: [M + H] ${ }^{+}$Calcd for $\mathrm{C}_{33} \mathrm{H}_{27} \mathrm{FN}_{3} \mathrm{O}_{3} \mathrm{~S}$ 564.1752; Found 564.1766. (11aS,11bS)-2-chloro-9,10-diphenyl-8-tosyl-8H,11bH-8a,11a-ethanofuro[3',2':3,4]pyrazolo[1,5-c]q uinazoline (5f)

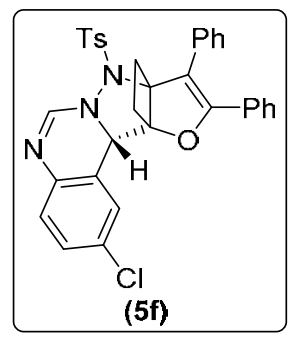

According to general procedure (GP2-B) with $\mathrm{PPh}_{3} \mathrm{AuCl}$ (1.1 mg, $2.2 \mu \mathrm{mol}, 1$ mol \%), $\operatorname{AgSbF}_{6} \quad(7.0 \quad \mathrm{mg}, 20 \mu \mathrm{mol}, 10 \quad \mathrm{~mol} \%)$, (6-chloroquinazolin-3-ium-3-yl)(tosyl)amide $3 f$ (73.5 mg, 0.220 mmol, 1.1 equiv), 3-cyclopropylidene-1,2-diphenylprop-2-en-1-one 1a (49.3 mg, 0.199 mmol, 1.0 equiv), $100 \mathrm{mg} 4 \AA \mathrm{MS}, 2.0 \mathrm{~mL}$ of dry 1,4-dioxane and $2.0 \mathrm{~mL}$ of dry DCE under $80{ }^{\circ} \mathrm{C}$ for 2 hours reaction. Purification by flash column chromatography provided $\mathbf{5 f}$ as a white solid (63.3 mg, 55\%); $d r=6: 1$ (ratio was determined by ${ }^{1} \mathrm{H}$ NMR analysis of the crude product); M.p. 130-132 ${ }^{\circ} \mathrm{C}$ (Petroleum ether/EtOAc); $\mathrm{R}_{f}=0.47$ (Petroleum ether/EtOAc $\left.=3 / 1\right) ;{ }^{1} \mathrm{H}$ NMR $(400 \mathrm{MHz}$, $\mathrm{CDCl}_{3}$ ): $\delta 7.57$ (d, $\left.J=8.0 \mathrm{~Hz}, 2 \mathrm{H}\right), 7.41$ (d, $\left.J=7.2 \mathrm{~Hz}, 2 \mathrm{H}\right), 7.37-7.28$ (m, 3H), 7.27-7.20 (m, 5H), 7.19-7.10 (m, 5H), 6.40 (s, 1H), 5.10 (s, 1H), 3.35-3.24 (m, 1H), 3.07-2.97 (m, 1H), 2.75-2.66 (m, 1H), 2.55-2.45 (m, 1H), 2.38 (s, 3H); ${ }^{13} \mathrm{C} \mathrm{NMR} \mathrm{(100} \mathrm{MHz,} \mathrm{CDCl}_{3}$ ): $\delta 157.3,151.0,144.9,138.4$, 134.8, 132.6, 131.4, 130.3, 129.7, 129.6, 129.4, 128.9, 128.6, 128.4, 128.2, 127.8, 127.5, 127.3, 126.2, 122.2, 111.6, 94.5, 80.9, 65.4, 25.6, 25.4, 21.6; HRMS (ESI) m/z: $[\mathrm{M}+\mathrm{H}]^{+}$Calcd for $\mathrm{C}_{33} \mathrm{H}_{27} \mathrm{ClN}_{3} \mathrm{O}_{3} \mathrm{~S}$ 580.1456; Found 580.1465.

(11aS,11bS)-2-bromo-9,10-diphenyl-8-tosyl-8H,11bH-8a,11a-ethanofuro[3',2':3,4]pyrazolo[1,5-c]q uinazoline (5g)

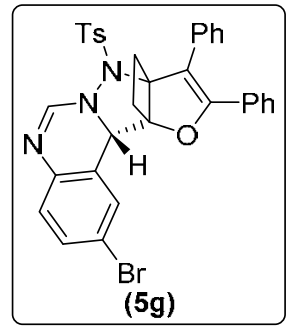

According to general procedure (GP2-B) with $\mathrm{PPh}_{3} \mathrm{AuCl}$ (1.1 mg, $2.2 \mu \mathrm{mol}, 1$

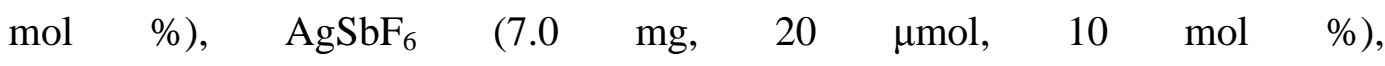
(6-bromoquinazolin-3-ium-3-yl)(tosyl)amide $3 g$ (83.4 mg, $0.220 \mathrm{mmol}, 1.1$ equiv), 3-cyclopropylidene-1,2-diphenylprop-2-en-1-one 1a (48.7 mg, 0.198 mmol, 1.0 equiv), $100 \mathrm{mg} 4 \AA \mathrm{MS}, 2.0 \mathrm{~mL}$ of dry 1,4-dioxane and $2.0 \mathrm{~mL}$ of dry DCE under $80{ }^{\circ} \mathrm{C}$ for 3.5 hours reaction. Purification by flash column chromatography provided $\mathbf{5 g}$ as a white solid (44.6 mg, 36\%); $d r=6: 1$ (ratio was determined by ${ }^{1} \mathrm{H}$ NMR analysis of the crude product); M.p. $182-184{ }^{\circ} \mathrm{C}$ (Petroleum ether/EtOAc); $\mathrm{R}_{f}=0.45$ (Petroleum ether/EtOAc $=3 / 1$ ); ${ }^{1} \mathrm{H}$ NMR (400 MHz, CDCl $): \delta 7.57$ (d, $J=8.4 \mathrm{~Hz}, 2 \mathrm{H}), 7.46-7.38$ (m, 3H), 7.36-7.27 (m, 4H), 7.26-7.13 (m, 7H), 7.08 (d, $J=8.4$ Hz, 1H), 6.41 (s, 1H), 5.11 (s, 1H), 3.38-3.24 (m, 1H), 3.08-2.97 
(m, 1H), 2.75-2.65 (m, 1H), 2.55-2.44 (m, 1H), 2.38 (s, 3H); ${ }^{13} \mathrm{C}$ NMR (100 MHz, $\left.\mathrm{CDCl}_{3}\right): \delta 157.3$, 151.1, 145.0, 138.8, 134.7, 132.5, 132.4, 130.2, 129.7, 129.6, 129.1, 128.8, 128.6, 128.4, 128.2, 127.8, 127.3, 122.5, 119.1, 111.5, 94.5, 80.8, 65.1, 25.5, 25.4, 21.6; HRMS (ESI) m/z: [M + H] Calcd for $\mathrm{C}_{33} \mathrm{H}_{27} \mathrm{BrN}_{3} \mathrm{O}_{3} \mathrm{~S}$ 624.0951; Found 624.0953.

(11aS,11bS)-9,10-diphenyl-8-tosyl-8H,11bH-8a,11a-ethano[1,3]dioxolo[4,5-g]furo[3',2':3,4]pyrazo lo[1,5-c]quinazoline (5h)

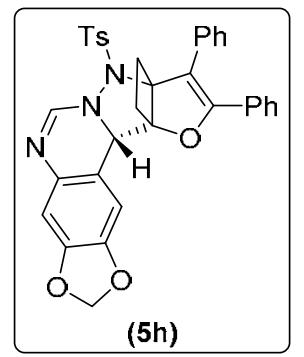

DCE under $80{ }^{\circ} \mathrm{C}$ for 3.5 hours reaction. Purification by flash column chromatography provided $\mathbf{5 h}$ as a white solid (79.3 mg, 66\%); $d r=4: 1$ (ratio was determined by ${ }^{1} \mathrm{H}$ NMR analysis of the crude product); M.p. $115-116{ }^{\circ} \mathrm{C}$ (Petroleum ether/EtOAc); $\mathrm{R}_{f}=0.37$ (Petroleum ether/EtOAc $=3 / 1$ ); ${ }^{1} \mathrm{H}$ NMR (400 MHz, $\mathrm{CDCl}_{3}$ ): $\delta 7.55$ (d, $\left.J=8.0 \mathrm{~Hz}, 2 \mathrm{H}\right), 7.45-7.28(\mathrm{~m}, 5 \mathrm{H}), 7.26-7.10(\mathrm{~m}, 7 \mathrm{H}), 6.73$ (s, 1H), 6.62 (s, 1H), 6.31 (s, 1H), 5.96 (d, $J=8.8 \mathrm{~Hz}, 2 \mathrm{H}), 5.03$ (s, 1H), 3.36-3.24 (m, 1H), 3.08-2.97 (m, 1H), 2.73-2.60 (m, 1H), 2.54-2.43 (m, 1H), 2.37 (s, 3H); ${ }^{13} \mathrm{C}$ NMR (100 MHz, $\left.\mathrm{CDCl}_{3}\right): \delta 157.3$, 149.4, 148.1, 145.7, 144.8, 134.8, 134.6, 132.7, 130.4, 129.6, 129.4, 128.8, 128.5, 128.4, 128.2, 127.8, 127.1, 113.3, 111.5, 107.3, 105.7, 101.4, 94.3, 80.7, 65.9, 25.6, 25.3, 21.6; HRMS (ESI) m/z: $[\mathrm{M}+\mathrm{H}]^{+}$Calcd for $\mathrm{C}_{34} \mathrm{H}_{28} \mathrm{~N}_{3} \mathrm{O}_{5} \mathrm{~S}$ 590.1744; Found 590.1747.

(11aS,11bS)-9,10-diphenyl-8-(thiophen-2-ylsulfonyl)-8H,11bH-8a,11a-ethanofuro[3',2':3,4]pyrazo lo[1,5-c]quinazoline (5i)

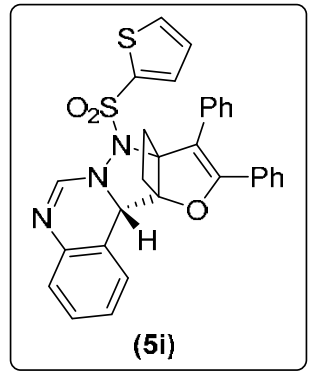

According to general procedure (GP2-A) with $\mathrm{PPh}_{3} \mathrm{AuCl}(1.2 \mathrm{mg}, 2.4 \mu \mathrm{mol}, 1$ mol \%), $\operatorname{AgSbF}_{6} \quad(7.5 \quad \mathrm{mg}, 22 \mu \mathrm{mol}, 10 \quad \mathrm{~mol} \%)$, quinazolin-3-ium-3-yl(thiophen-2-ylsulfonyl)amide 3i (64.5 mg, 0.222 mmol, 1.1 equiv), 3-cyclopropylidene-1,2-diphenylprop-2-en-1-one 1a (49.6 mg, 0.202 mmol, 1.0 equiv), $100 \mathrm{mg} 4 \AA \mathrm{MS}, 2.0 \mathrm{~mL}$ of dry EA for 12 hours reaction.

Purification by flash column chromatography provided $5 \mathbf{i}$ as a white solid $(79.8 \mathrm{mg}, 74 \%) ; d r=8: 1$ (ratio was determined by ${ }^{1} \mathrm{H}$ NMR analysis of the crude product); M.p. 202-203 ${ }^{\circ} \mathrm{C}$ (Petroleum ether/EtOAc); $\mathrm{R}_{f}=0.32($ Petroleum ether/EtOAc $=3 / 1) ;{ }^{1} \mathrm{H}$ NMR $\left(400 \mathrm{MHz}, \mathrm{CDCl}_{3}\right): \delta$ 7.66-7.55 
(m, 2H), 7.40 (d, $J=7.2 \mathrm{~Hz}, 2 \mathrm{H}), 7.37-7.28$ (m, 5H), 7.23-7.69 (m, 7H), 7.05-6.97 (m, 1H), 6.68 (s, 1H), 5.07 (s, 1H), 3.34-3.13 (m, 1H), 3.02-2.91 (m, 1H), 2.72-2.60 (m, 1H), 2.53-2.41 (m, $1 \mathrm{H}) ;{ }^{13} \mathrm{C}$ NMR (100 MHz, $\left.\mathrm{CDCl}_{3}\right): \delta$ 157.3, 150.7, 139.5, 138.1, 135.0, 134.5, 132.4, 130.2, 129.43, 129.37, 129.0, 128.4, 128.1, 127.8, 127.35, 127.26, 126.4, 126.3, 126.2, 120.4, 111.4, 94.4, 81.1, 65.9, 25.29, 25.26; HRMS (ESI) m/z: [M + H] $]^{+}$Calcd for $\mathrm{C}_{30} \mathrm{H}_{24} \mathrm{~N}_{3} \mathrm{O}_{3} \mathrm{~S}_{2}$ 538.1254; Found 538.1263.

(11aS,11bS)-8-((4-fluorophenyl)sulfonyl)-9,10-diphenyl-8H,11bH-8a,11a-ethanofuro[3',2':3,4]pyr azolo[1,5-c]quinazoline (5j)

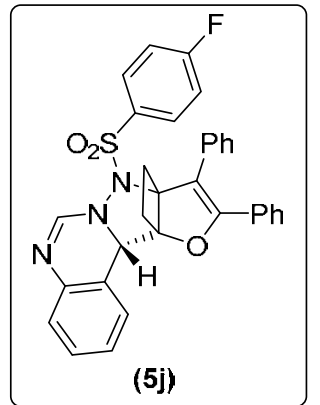

According to general procedure (GP2-A) with $\mathrm{PPh}_{3} \mathrm{AuCl}$ (1.0 mg, $2.0 \mu \mathrm{mol}, 1$ mol \%), $\mathrm{AgSbF}_{6} \quad(7.4 \quad \mathrm{mg}, 22 \mu \mathrm{mol}, 10 \mathrm{~mol} \%)$, ((4-fluorophenyl)sulfonyl)(quinazolin-3-ium-3-yl)amide $3 \mathbf{3}$ (66.2 mg, 0.218 mmol, 1.1 equiv), 3-cyclopropylidene-1,2-diphenylprop-2-en-1-one 1a (49.5 mg, $0.201 \mathrm{mmol}, 1.0$ equiv), $100 \mathrm{mg} 4 \AA \mathrm{MS}, 2.0 \mathrm{~mL}$ of dry EA for 12 hours reaction. Purification by flash column chromatography provided 5j as a white solid (88.4 mg, 80\%); $d r=11$ : 1 (ratio was determined by ${ }^{1} \mathrm{H}$ NMR analysis of the crude product); M.p. 131-132 ${ }^{\circ} \mathrm{C}$ (Petroleum ether/EtOAc); $\mathrm{R}_{f}=0.66$ (Petroleum ether/EtOAc $\left.=3 / 1\right) ;{ }^{1} \mathrm{H} \mathrm{NMR}(400 \mathrm{MHz}$, $\mathrm{CDCl}_{3}$ ): $\delta$ 7.71-7.61 (m, 2H), 7.40 (d, $\left.J=6.8 \mathrm{~Hz}, 2 \mathrm{H}\right), 7.37-7.28(\mathrm{~m}, 4 \mathrm{H}), 7.25-7.12(\mathrm{~m}, 8 \mathrm{H}), 7.08$ (t, $J=8.4 \mathrm{~Hz}, 2 \mathrm{H}), 6.40$ (s, 1H), 5.13 (s, 1H), 3.35-3.24 (m, 1H), 3.12-3.01 (m, 1H), 2.76-2.66 (m, 1H), 2.55-2.41 (m, 1H); ${ }^{13} \mathrm{C}$ NMR (100 MHz, $\left.\mathrm{CDCl}_{3}\right): \delta 165.6$ (d, $\left.J=255.5 \mathrm{~Hz}\right), 157.5,150.5,139.5$, 133.8, 132.7, 131.4 (d, $J=9.3 \mathrm{~Hz}), 130.2,129.5,129.4,128.8,128.4,128.1,127.8,127.2,126.5$, 126.3, 126.2, 120.4, 116.3 (d, $J=22.8 \mathrm{~Hz}$ ), 111.3, 94.4, 81.0, 65.9, 25.8, 25.4; HRMS (ESI) m/z: [M $+\mathrm{H}]^{+}$Calcd for $\mathrm{C}_{32} \mathrm{H}_{25} \mathrm{FN}_{3} \mathrm{O}_{3} \mathrm{~S}$ 550.1595; Found 550.1599.

(11aS,11bS)-8-((4-chlorophenyl)sulfonyl)-9,10-diphenyl-8H,11bH-8a,11a-ethanofuro[3',2':3,4]pyr azolo[1,5-c]quinazoline (5k)

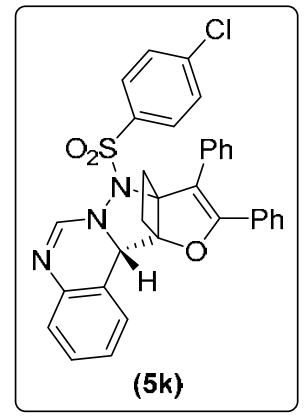

According to general procedure (GP2-A) with $\mathrm{PPh}_{3} \mathrm{AuCl}(1.1 \mathrm{mg}, 2.2 \mu \mathrm{mol}, 1$ mol \%), $\operatorname{AgSbF}_{6} \quad(6.8 \quad \mathrm{mg}, 20 \mu \mathrm{mol}, 10 \quad \mathrm{~mol} \%)$, ((4-chlorophenyl)sulfonyl)(quinazolin-3-ium-3-yl)amide $3 \mathbf{k} \quad(71.0 \mathrm{mg}, 0.223$ mmol, 1.1 equiv), 3-cyclopropylidene-1,2-diphenylprop-2-en-1-one 1a (49.4 mg, $0.201 \mathrm{mmol}, 1.0$ equiv), $100 \mathrm{mg} 4 \AA \mathrm{MS}, 2.0 \mathrm{~mL}$ of dry EA for 5.5 hours reaction. Purification by flash column chromatography provided 5k as a pale yellow solid (91.9 mg, 81\%); $d r=9: 1$ (ratio was determined by ${ }^{1} \mathrm{H}$ NMR analysis of the crude product); M.p. 
160-162 ${ }^{\circ} \mathrm{C}$ (Petroleum ether/EtOAc); $\mathrm{R}_{f}=0.47$ (Petroleum ether/EtOAc $\left.=3 / 1\right) ;{ }^{1} \mathrm{H}$ NMR $(400 \mathrm{MHz}$, $\mathrm{CDCl}_{3}$ ): $\delta 7.57$ (d, $\left.J=8.4 \mathrm{~Hz}, 2 \mathrm{H}\right), 7.42-7.35$ (m, 4H), 7.34-7.27 (m, 4H), 7.26-7.10 (m, 8H), 6.40 (s, $1 \mathrm{H}), 5.12$ (s, 1H), 3.33-3.23 (m, 1H), 3.12-3.04 (m, 1H), 2.75-2.66 (m, 1H), 2.54-2.43 (m, $1 \mathrm{H}) ;{ }^{13} \mathrm{C}$ NMR (100 MHz, $\left.\mathrm{CDCl}_{3}\right): \delta 157.5,150.5,140.4,139.5,136.3,132.7,130.2,129.9,129.5,129.4$, 129.3, 128.8, 128.4, 128.2, 127.8, 127.2, 126.5, 126.3, 126.2, 120.3, 111.2, 94.4, 81.1, 65.9, 25.9, 25.5; HRMS (ESI) m/z: [M + H] ${ }^{+}$Calcd for $\mathrm{C}_{32} \mathrm{H}_{25} \mathrm{ClN}_{3} \mathrm{O}_{3} \mathrm{~S}$ 566.1300; Found 566.1305.

(11aS,11bS)-8-((4-methoxyphenyl)sulfonyl)-9,10-diphenyl-8H,11bH-8a,11a-ethanofuro[3',2':3,4]p yrazolo[1,5-c]quinazoline (5l)

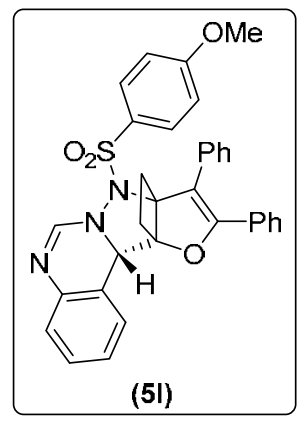

According to general procedure (GP2-A) with $\mathrm{PPh}_{3} \mathrm{AuCl}(1.0 \mathrm{mg}, 2.0 \mu \mathrm{mol}, 1$ mol \%), $\operatorname{AgSbF}_{6} \quad(7.3 \quad \mathrm{mg}, 21 \mu \mathrm{mol}, 10 \quad \mathrm{~mol} \%)$, ((4-methoxyphenyl)sulfonyl)(quinazolin-3-ium-3-yl)amide 31 (69.9 mg, 0.222 mmol, 1.1 equiv), 3-cyclopropylidene-1,2-diphenylprop-2-en-1-one 1a (49.3 mg, $0.200 \mathrm{mmol}, 1.0$ equiv), $100 \mathrm{mg} 4 \AA \mathrm{MS}, 2.0 \mathrm{~mL}$ of dry EA for 3.5 hours reaction. Purification by flash column chromatography provided $\mathbf{5 l}$ as a pale yellow solid (77.7 mg, 73\%); $d r=9: 1$ (ratio was determined by ${ }^{1} \mathrm{H}$ NMR analysis of the crude product); M.p. 163-164 ${ }^{\circ} \mathrm{C}$ (Petroleum ether/EtOAc); $\mathrm{R}_{f}=0.27$ (Petroleum ether/EtOAc $\left.=3 / 1\right) ;{ }^{1} \mathrm{H}$ NMR $(400 \mathrm{MHz}$, $\left.\mathrm{CDCl}_{3}\right): \delta 7.61$ (d, $\left.J=9.2 \mathrm{~Hz}, 2 \mathrm{H}\right), 7.45-7.38$ (m, 2H), 7.37-7.26 (m, 4H), 7.23-7.10 (m, 8H), 6.86 (d, $J=9.2 \mathrm{~Hz}, 2 \mathrm{H}), 6.44$ (s, 1H), 5.16 (s, 1H), 3.79 (s, 3H), 3.37-3.26 (m, 1H), 3.06-2.92 (m, 1H), 2.75-2.65 (m, 1H), 2.53-2.42 (m, 1H); $\left.{ }^{13} \mathrm{C} \mathrm{NMR} \mathrm{(100} \mathrm{MHz,} \mathrm{CDCl}_{3}\right): \delta$ 163.7, 157.3, 150.9, 139.6, 132.7, 130.8, 130.4, 129.4, 129.3, 129.2, 128.9, 128.3, 128.1, 127.8, 127.1, 126.3, 126.21, 126.19, 120.5, 114.2, 111.6, 94.6, 80.7, 65.7, 55.6, 25.5, 25.4; HRMS (ESI) m/z: $[\mathrm{M}+\mathrm{H}]^{+}$Calcd for $\mathrm{C}_{33} \mathrm{H}_{28} \mathrm{~N}_{3} \mathrm{O}_{4} \mathrm{~S}$ 562.1795; Found 562.1798.

(11aS,11bS)-8-(naphthalen-2-ylsulfonyl)-9,10-diphenyl-8H,11bH-8a,11a-ethanofuro[3',2':3,4]pyra zolo[1,5-c]quinazoline (5m)

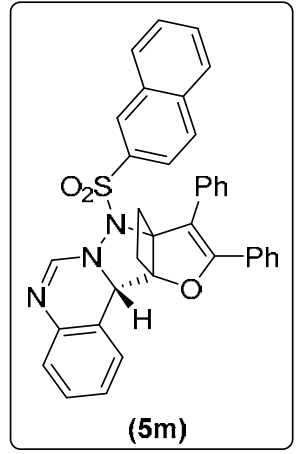

According to general procedure (GP2-A) with $\mathrm{PPh}_{3} \mathrm{AuCl}$ (1.1 mg, $2.2 \mu \mathrm{mol}, 1$ mol \%), $\operatorname{AgSbF}_{6} \quad(6.8 \quad \mathrm{mg}, 20 \mu \mathrm{mol}, 10 \mathrm{~mol} \%)$, (naphthalen-2-ylsulfonyl)(quinazolin-3-ium-3-yl)amide $3 \mathrm{~m} \quad$ (74.1 mg, 0.221 mmol, 1.1 equiv), 3-cyclopropylidene-1,2-diphenylprop-2-en-1-one 1a (49.3 mg, $0.200 \mathrm{mmol}, 1.0$ equiv), $100 \mathrm{mg} 4 \AA \mathrm{MS}, 2.0 \mathrm{~mL}$ of dry EA for 12 hours reaction. 
Purification by flash column chromatography provided $5 \mathrm{~m}$ as a white solid (83.8 $\mathrm{mg}, 72 \%) ; d r=9$ : 1 (ratio was determined by ${ }^{1} \mathrm{H}$ NMR analysis of the crude product); M.p. $130-132{ }^{\circ} \mathrm{C}$ (Petroleum ether/EtOAc); $\mathrm{R}_{f}=0.52$ (Petroleum ether/EtOAc = 3/1); ${ }^{1} \mathrm{H}$ NMR $\left(400 \mathrm{MHz}, \mathrm{CDCl}_{3}\right): \delta 8.31(\mathrm{~s}, 1 \mathrm{H})$, 7.90-7.77 (m, 3H), 7.68-7.31 (m, 9H), 7.24-7.08 (m, 8H), 6.35 (s, 1H), 5.19 (s, 1H), 3.43-3.30 (m, 1H), 3.16-3.05 (m, 1H), 2.80-2.68 (m, 1H), 2.58-2.44 (m, 1H); ${ }^{13} \mathrm{C}$ NMR (100 MHz, $\left.\mathrm{CDCl}_{3}\right): \delta 157.4$, 150.7, 139.5, 135.2, 134.6, 132.8, 131.7, 130.4, 130.3, 129.44, 129.41, 129.3, 129.0, 128.4, 128.1, 127.8, 127.6, 127.3, 126.4, 126.22, 126.21, 123.0, 120.5, 111.5, 94.5, 81.0, 65.9, 26.0, 25.5; HRMS (ESI) $\mathrm{m} / \mathrm{z}:[\mathrm{M}+\mathrm{H}]^{+}$Calcd for $\mathrm{C}_{36} \mathrm{H}_{28} \mathrm{~N}_{3} \mathrm{O}_{3} \mathrm{~S}$ 582.1846; Found 582.1859.

(11aS,11bS)-10-(4-fluorophenyl)-9-phenyl-8-tosyl-8H,11bH-8a,11a-ethanofuro[3',2':3,4]pyrazolo[

\section{1,5-clquinazoline (5n)}

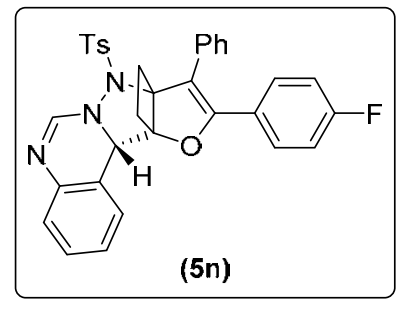

According to general procedure (GP2-A) with $\mathrm{PPh}_{3} \mathrm{AuCl}(1.1 \mathrm{mg}, 2.2 \mu \mathrm{mol}$, $1 \mathrm{~mol} \mathrm{\% ),} \operatorname{AgSbF}_{6} \quad(7.1 \mathrm{mg}, 21 \mu \mathrm{mol}, 10 \mathrm{~mol} \%)$, quinazolin-3-ium-3-yl(tosyl)amide 3a (66.2 mg, $0.22 \mathrm{mmol}, 1.1$ equiv), 3-cyclopropylidene-1-(4-fluorophenyl)-2-phenylprop-2-en-1-one $\mathbf{1 b}$ (53.5 mg, 0.203 mmol, 1.0 equiv), $100 \mathrm{mg} 4 \AA$ MS, $2.0 \mathrm{~mL}$ of dry EA for 12 hours reaction. Purification by flash column chromatography provided 5n as a white solid (90.1 mg, 79\%); $d r=9$ : 1 (ratio was determined by ${ }^{1} \mathrm{H}$ NMR analysis of the crude product); M.p. 202-203 ${ }^{\circ} \mathrm{C}$ (Petroleum ether/EtOAc); $\mathrm{R}_{f}=0.42$ (Petroleum ether/EtOAc $\left.=3 / 1\right) ;{ }^{1} \mathrm{H}$ NMR (400 MHz, $\left.\mathrm{CDCl}_{3}\right): \delta 7.56(\mathrm{~d}, J=8.4 \mathrm{~Hz}, 2 \mathrm{H})$, 7.43-7.29 (m, 6H), 7.25-7.10 (m, 7H), 6.83 (t, $J=8.8$ Hz, 2H), 6.40 (s, 1H), 5.15 (s, 1H), 3.36-3.25 (m, 1H), 3.06-2.92 (m, 1H), 2.76-2.64 (m, 1H), 2.53-2.42 (m, 1H), 2.38 (s, 3H); ${ }^{13} \mathrm{C}$ NMR (100 MHz, $\left.\mathrm{CDCl}_{3}\right): \delta 163.1(\mathrm{~d}, J=248.7 \mathrm{~Hz}), 156.2,150.8,144.8,139.5,134.8,132.5,129.9,129.8,129.6$, 129.3, 128.9, 128.6, 128.5, 127.3, 126.4, 126.2, 120.4, 115.3 (d, $J=21.7 \mathrm{~Hz}$ ), 111.4, 94.6, 80.8, 65.7, 25.5, 25.4, 21.5; HRMS (ESI) m/z: [M + H] ${ }^{+}$Calcd for $\mathrm{C}_{33} \mathrm{H}_{27} \mathrm{FN}_{3} \mathrm{O}_{3} \mathrm{~S}$ 564.1752; Found 564.1760.

(11aS,11bS)-9,10-diphenyl-8-tosyl-8H,11bH-8a,11a-ethanofuro[3',2':3,4]pyrazolo[1,5-c]quinazoli ne (5o)

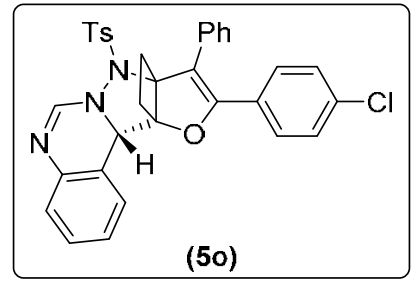

According to general procedure (GP2-A) with $\mathrm{PPh}_{3} \mathrm{AuCl}(0.9 \mathrm{mg}, 1.8$ $\mu \mathrm{mol}, 1 \mathrm{~mol} \%), \operatorname{AgSbF}_{6}(7.2 \mathrm{mg}, 21 \mu \mathrm{mol}, 10 \mathrm{~mol} \%)$, quinazolin-3-ium-3-yl(tosyl)amide 3a (65.7 mg, $0.220 \mathrm{mmol}, 1.1$ equiv), 1-(4-chlorophenyl)-3-cyclopropylidene-2-phenylprop-2-en-1-one 1c (55.8

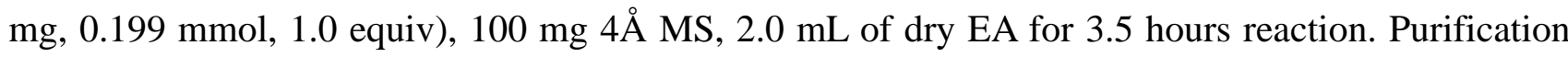


by flash column chromatography provided 50 as a white solid (93.4 mg, 81\%); $d r=11$ : 1 (ratio was determined by ${ }^{1} \mathrm{H}$ NMR analysis of the crude product); M.p. 202-203 ${ }^{\circ} \mathrm{C}$ (Petroleum ether/EtOAc); $\mathrm{R}_{f}=0.43$ (Petroleum ether/EtOAc $\left.=3 / 1\right) ;{ }^{1} \mathrm{H}$ NMR (400 MHz, $\left.\mathrm{CDCl}_{3}\right): \delta 7.56(\mathrm{~d}, J=8.4 \mathrm{~Hz}, 2 \mathrm{H})$, 7.44-7.25 (m, 6H), 7.24-7.15 (m, 5H), 7.08 (s, 4H), 6.40 (s, 1H), 5.14 (s, 1H), 3.40-3.22 (m, 1H), 3.10-2.97 (m, 1H), 2.74-2.65 (m, 1H), 2.52-2.40 (m, 1H), $2.36(\mathrm{~s}, 1 \mathrm{H}) ;{ }^{13} \mathrm{C}$ NMR (100 MHz, CDCl $)$ : $\delta$ 156.0, 150.8. 144.8. 139.5. 135.3, 134.8, 132.4, 129.6, 129.4, 129.1, 128.9, 128.7, 128.6, 128.5, 128.4, 127.5, 126.4, 126.23, 126.19, 120.4, 112.1, 94.6, 80.7, 65.7, 25.5, 25.4, 21.5; HRMS (ESI) $\mathrm{m} / \mathrm{z}:[\mathrm{M}+\mathrm{H}]^{+}$Calcd for $\mathrm{C}_{33} \mathrm{H}_{27} \mathrm{ClN}_{3} \mathrm{O}_{3} \mathrm{~S}$ 580.1456; Found 580.1462.

(11aS,11bS)-10-(4-bromophenyl)-9-phenyl-8-tosyl-8H,11bH-8a,11a-ethanofuro[3',2':3,4]pyrazolo[

\section{1,5-clquinazoline (5p)}

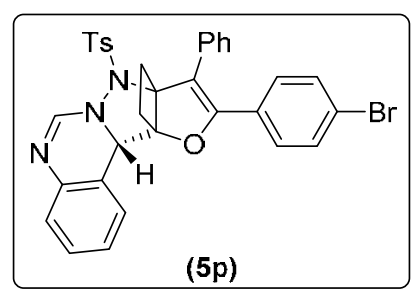

(5p)
According to general procedure (GP2-A) with $\mathrm{PPh}_{3} \mathrm{AuCl}(1.1 \mathrm{mg}, 2.2$ $\mu \mathrm{mol}, 1 \mathrm{~mol} \%), \mathrm{AgSbF}_{6} \quad(7.0 \mathrm{mg}, 20 \mu \mathrm{mol}, 10 \mathrm{~mol} \%)$, quinazolin-3-ium-3-yl(tosyl)amide 3a (65.2 mg, $0.218 \mathrm{mmol}, 1.1$ equiv), 1-(4-bromophenyl)-3-cyclopropylidene-2-phenylprop-2-en-1-one 1d (65.2

mg, $0.201 \mathrm{mmol}, 1.0$ equiv), $100 \mathrm{mg} 4 \AA \mathrm{MS}, 2.0 \mathrm{~mL}$ of dry EA for 3.5 hours reaction. Purification by flash column chromatography provided 5p as a white solid (95.6 mg, 76\%); $d r=11$ : 1 (ratio was determined by ${ }^{1} \mathrm{H}$ NMR analysis of the crude product); M.p. $210-212{ }^{\circ} \mathrm{C}$ (Petroleum ether/EtOAc); $\mathrm{R}_{f}=0.41$ (Petroleum ether/EtOAc $\left.=3 / 1\right) ;{ }^{1} \mathrm{H}$ NMR (400 MHz, $\left.\mathrm{CDCl}_{3}\right): \delta 7.56$ (d, $\left.J=8.4 \mathrm{~Hz}, 2 \mathrm{H}\right)$, 7.44-7.23 (m, 8H), 7.22-7.14 (m, 5H), 7.07-7.00 (m, 2H), 6.40 (s, 1H), 5.14 (s, 1H), 3.38-3.22 (m, 1H), 3.05-2.94 (m, 1H), 2.75-2.65 (m, 1H), 2.52-2.40 (m, 1H), 2.36 (s, 3H); ${ }^{13} \mathrm{C}$ NMR (100 MHz, $\left.\mathrm{CDCl}_{3}\right): \delta 156.0,150.7,144.8,139.5,134.8,132.3,131.4,129.6,129.3,129.2,128.9,128.6,128.5$, 127.5, 126.4, 126.22, 126.18, 123.6, 120.4, 112.2, 94.6, 80.7, 65.7, 25.43, 25.36, 21.5; HRMS (ESI) $\mathrm{m} / \mathrm{z}:[\mathrm{M}+\mathrm{H}]^{+}$Calcd for $\mathrm{C}_{33} \mathrm{H}_{27} \mathrm{BrN}_{3} \mathrm{O}_{3} \mathrm{~S}$ 624.0951; Found 624.0955.

(11aS,11bS)-10-(2-fluorophenyl)-9-phenyl-8-tosyl-8H,11bH-8a,11a-ethanofuro[3',2':3,4]pyrazolo[

\section{1,5-c]quinazoline (5q)}

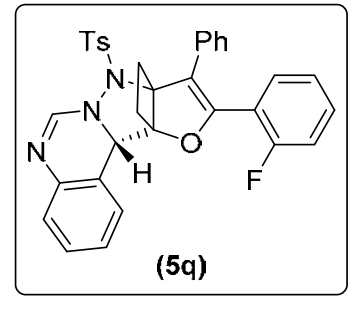

According to general procedure (GP2-A) with $\mathrm{PPh}_{3} \mathrm{AuCl}(1.2 \mathrm{mg}, 2.4 \mu \mathrm{mol}, 1$ mol \%), $\operatorname{AgSbF}_{6} \quad(6.5 \quad \mathrm{mg}, 19 \mu \mathrm{mol}, 10 \mathrm{~mol} \%)$, quinazolin-3-ium-3-yl(tosyl)amide 3a (65.2 mg, $0.220 \mathrm{mmol}, 1.1$ equiv), 3-cyclopropylidene-1-(2-fluorophenyl)-2-phenylprop-2-en-1-one 1f (53.2 mg,

$0.202 \mathrm{mmol}, 1.0$ equiv), $100 \mathrm{mg} 4 \AA \mathrm{MS}, 2.0 \mathrm{~mL}$ of dry EA for 12 hours reaction. Purification by 
flash column chromatography provided $5 \mathbf{q}$ as a white solid (62.5 $\mathrm{mg}, 55 \%$ ); $d r=8: 1$ (ratio was determined by ${ }^{1} \mathrm{H}$ NMR analysis of the crude product); M.p. $188-189{ }^{\circ} \mathrm{C}$ (Petroleum ether/EtOAc); $\mathrm{R}_{f}=0.45$ (Petroleum ether/EtOAc $\left.=3 / 1\right) ;{ }^{1} \mathrm{H}$ NMR (400 MHz, $\left.\mathrm{CDCl}_{3}\right): \delta 7.63(\mathrm{~d}, J=8.4 \mathrm{~Hz}, 2 \mathrm{H})$, 7.40 (d, $J=7.2 \mathrm{~Hz}, 2 \mathrm{H}), 7.32-7.13(\mathrm{~m}, 11 \mathrm{H}), 7.03$ (t, $J=7.4 \mathrm{~Hz}, 1 \mathrm{H}), 6.95$ (t, $J=8.8 \mathrm{~Hz}, 1 \mathrm{H}), 6.44$ (s, $1 \mathrm{H}), 5.20$ (s, 1H), 3.44-3.34 (m, 1H), 3.12-3.01 (m, 1H), 2.77-2.68 (m, 1H), 2.58-2.48 (m, 1H), 2.37 (s, 3H); ${ }^{13} \mathrm{C}$ NMR (100 MHz, $\left.\mathrm{CDCl}_{3}\right): \delta 159.6$ (d, $\left.J=251.5 \mathrm{~Hz}\right), 152.4,150.7,144.9,139.6$, 134.7, 132.0, 131.4 (d, $J=7.9 \mathrm{~Hz}$ ), 130.3 (d, $J=1.6 \mathrm{~Hz}), 129.7,129.3,128.6,128.2,127.3,126.9$, 126.4, 126.2 (d, $J=2.8 \mathrm{~Hz}), 124.3$ (d, $J=3.1 \mathrm{~Hz}), 120.4,119.2$ (d, $J=14.7 \mathrm{~Hz}), 116.1$ (d, $J=21.0$ $\mathrm{Hz}), 114.7,95.2$, 80.3, 65.6, 25.6, 25.3, 21.6; HRMS (ESI) m/z: $[\mathrm{M}+\mathrm{H}]^{+}$Calcd for $\mathrm{C}_{33} \mathrm{H}_{27} \mathrm{FN}_{3} \mathrm{O}_{3} \mathrm{~S}$ 564.1752; Found 564.1751.

(11aS,11bS)-10-(2-bromophenyl)-9-phenyl-8-tosyl-8H,11bH-8a,11a-ethanofuro[3',2':3,4]pyrazolo[

\section{1,5-c]quinazoline (5r)}

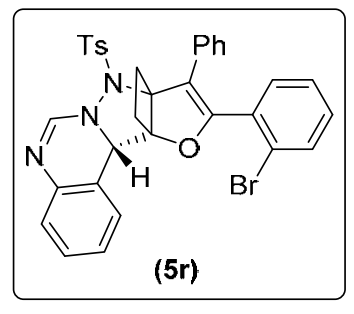

According to general procedure (GP2-A) with $\mathrm{PPh}_{3} \mathrm{AuCl}(1.1 \mathrm{mg}, 2.2 \mu \mathrm{mol}, 1$ mol \%), $\operatorname{AgSbF}_{6} \quad(6.9 \quad \mathrm{mg}, 20 \mu \mathrm{mol}, 10 \mathrm{~mol} \%)$, quinazolin-3-ium-3-yl(tosyl)amide 3a (65.9 mg, $0.220 \mathrm{mmol}, 1.1$ equiv), 1-(2-bromophenyl)-3-cyclopropylidene-2-phenylprop-2-en-1-one 1 g (65.5 mg,

$0.202 \mathrm{mmol}, 1.0$ equiv), $100 \mathrm{mg} 4 \AA \mathrm{MS}, 2.0 \mathrm{~mL}$ of dry EA for 12 hours reaction. Purification by flash column chromatography provided $5 r$ as a white solid (80.1 mg, 64\%); $d r=5: 1$ (ratio was determined by ${ }^{1} \mathrm{H}$ NMR analysis of the crude product); M.p. 219-220 ${ }^{\circ} \mathrm{C}$ (Petroleum ether/EtOAc); $\mathrm{R}_{f}=0.30$ (Petroleum ether/EtOAc = 3/1); ${ }^{1} \mathrm{H}$ NMR (400 MHz, $\left.\mathrm{CDCl}_{3}\right): \delta 7.74(\mathrm{~d}, J=6.8 \mathrm{~Hz}, 2 \mathrm{H})$, 7.53-7.45 (m, 1H), 7.41-7.34 (m, 2H), 7.28-7.08 (m, 12H), 6.50 (s, 1H), 5.25 (s, 1H), 3.48-3.36 (m, 1H), 3.10-3.00 (m, 1H), 2.77-2.60 (m, 2H), 2.37 (s, 3H); ${ }^{13} \mathrm{C}$ NMR (100 MHz, $\left.\mathrm{CDCl}_{3}\right): \delta 156.9$, 150.6, 145.0, 139.5, 134.6, 133.0, 132.6, 131.3, 131.0, 130.8, 129.7, 129.3, 128.7, 128.4, 127.8, 126.7, 126.6, 126.4, 126.3, 123.2, 120.3, 113.9, 95.7, 79.9, 65.3, 25.4, 25.1, 21.6; HRMS (ESI) m/z: $[\mathrm{M}+\mathrm{H}]^{+}$Calcd for $\mathrm{C}_{33} \mathrm{H}_{27} \mathrm{BrN}_{3} \mathrm{O}_{3} \mathrm{~S}$ 624.0951; Found 624.0956.

(11aS,11bS)-9-phenyl-10-(p-tolyl)-8-tosyl-8H,11bH-8a,11a-ethanofuro[3',2':3,4]pyrazolo[1,5-c]qui nazoline (5s)

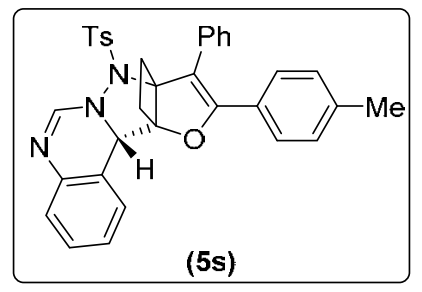

According to general procedure (GP2-A) with $\mathrm{PPh}_{3} \mathrm{AuCl}$ (1.2 mg, 2.4 $\mu \mathrm{mol}, 1 \mathrm{~mol} \%), \mathrm{AgSbF}_{6} \quad(7.3 \mathrm{mg}, 21 \mu \mathrm{mol}, 10 \mathrm{~mol} \%)$, 
quinazolin-3-ium-3-yl(tosyl)amide $\quad$ 3a $\quad(63.2 \quad \mathrm{mg}, \quad 0.211 \quad \mathrm{mmol}, \quad 1.1 \quad$ equiv), 3-cyclopropylidene-2-phenyl-1-(p-tolyl)prop-2-en-1-one $\mathbf{1 h}$ (52.5 mg, $0.202 \mathrm{mmol}, 1.0$ equiv), 100

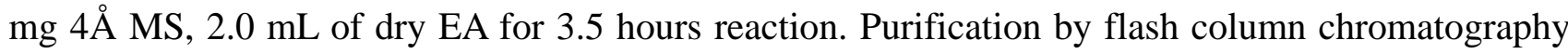
provided 5s as a white solid (92.4 mg, 82\%); $d r=9: 1$ (ratio was determined by ${ }^{1} \mathrm{H}$ NMR analysis of the crude product); M.p. $162-163{ }^{\circ} \mathrm{C}$ (Petroleum ether/EtOAc); $\mathrm{R}_{f}=0.41$ (Petroleum ether/EtOAc $=$ 3/1); ${ }^{1} \mathrm{H}$ NMR (400 MHz, $\mathrm{CDCl}_{3}$ ): $\delta 7.57$ (d, $\left.J=8.0 \mathrm{~Hz}, 2 \mathrm{H}\right), 7.43$ (d, $\left.J=7.2 \mathrm{~Hz}, 2 \mathrm{H}\right), 7.37-7.24$ (m, 4H), 7.23-7.13 (m, 5H), 7.07 (d, $J=8.0 \mathrm{~Hz}, 2 \mathrm{H}), 6.95$ (d, $J=8.0 \mathrm{~Hz}, 2 \mathrm{H}), 6.40$ (s, 1H), 5.14 (s, 1H), 3.36-3.22 (m, 1H), 3.05-2.94 (m, 1H), 2.75-2.64 (m, 1H), 2.52-2.40 (m, 1H), 2.35 (s, 3H), 2.24 (s, 3H); ${ }^{13} \mathrm{C}$ NMR (100 MHz, $\left.\mathrm{CDCl}_{3}\right): \delta$ 157.5, 150.8, 144.7, 139.6, 139.5, 134.9, 132.9, 129.6, 129.2, 128.84, 128.80, 128.6, 128.3, 127.7, 127.5, 127.0, 126.3, 126.19, 126.18, 120.5, 110.8, 94.4, 80.8, 65.7, 25.6, 25.4, 21.5, 21.3; HRMS (ESI) m/z: $[\mathrm{M}+\mathrm{H}]^{+}$Calcd for $\mathrm{C}_{34} \mathrm{H}_{30} \mathrm{~N}_{3} \mathrm{O}_{3} \mathrm{~S}$ 560.2002; Found 560.2014.

(11aS,11bS)-10-(4-methoxyphenyl)-9-phenyl-8-tosyl-8H,11bH-8a,11a-ethanofuro[3',2':3,4]pyrazol o[1,5-c]quinazoline (5t)

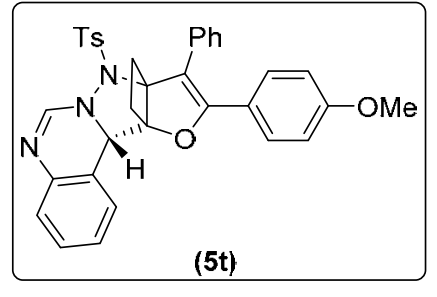

According to general procedure (GP2-A) with $\mathrm{PPh}_{3} \mathrm{AuCl}(1.1 \mathrm{mg}, 2.2$ $\mu \mathrm{mol}, 1 \mathrm{~mol} \%), \mathrm{AgSbF}_{6}(6.7 \mathrm{mg}, 19 \mu \mathrm{mol}, 10 \mathrm{~mol} \%)$, quinazolin-3-ium-3-yl(tosyl)amide 3a (65.7 mg, 0.220 mmol, 1.1 equiv),

3-cyclopropylidene-1-(4-methoxyphenyl)-2-phenylprop-2-en-1-one $\quad \mathbf{1 i}$

(55.8 mg, $0.202 \mathrm{mmol}, 1.0$ equiv), $100 \mathrm{mg} 4 \AA \mathrm{MS}, 2.0 \mathrm{~mL}$ of dry EA for 12 hours reaction. Purification by flash column chromatography provided 5t as a white solid (68.1 mg, 59\%); $d r=10$ : 1 (ratio was determined by ${ }^{1} \mathrm{H}$ NMR analysis of the crude product); M.p. $192-193{ }^{\circ} \mathrm{C}$ (Petroleum ether/EtOAc); $\mathrm{R}_{f}=0.37$ (Petroleum ether/EtOAc = 3/1); ${ }^{1} \mathrm{H}$ NMR (400 MHz, $\left.\mathrm{CDCl}_{3}\right): \delta 7.56(\mathrm{~d}, J=$ 8.4 Hz, 2H), 7.43 (d, $J=7.2 \mathrm{~Hz}, 2 \mathrm{H}), 7.38-7.26$ (m, 4H), 7.23-7.15 (m, 5H), 7.12 (d, $J=8.8 \mathrm{~Hz}, 2 \mathrm{H}$ ), 6.67 (d, $J=8.8 \mathrm{~Hz}, 2 \mathrm{H}), 6.39$ (s, 1H), 5.14 (s, 1H), 3.71 (s, 3H), 3.35-3.24 (m, 1H), 3.06-2.94 (m, 1H), 2.74-2.64 (m, 1H), 2.52-2.40 (m, 1H), 2.36 (s, 3H); ${ }^{13} \mathrm{C}$ NMR (100 MHz, CDCl $): \delta 160.4$, 157.3, 150.9, 144.7, 139.6, 134.9, 133.0, 129.6, 129.30, 129.25, 128.9, 128.6, 128.3, 127.0, 126.3, 126.22, 126.16, 122.7, 120.6, 113.5, 110.0, 94.3, 80.9, 65.7, 55.1, 25.6, 25.4, 21.5; HRMS (ESI) m/z: $[\mathrm{M}+\mathrm{H}]^{+}$Calcd for $\mathrm{C}_{34} \mathrm{H}_{30} \mathrm{~N}_{3} \mathrm{O}_{4} \mathrm{~S}$ 576.1952; Found 576.1964.

1-(4-((11aS,11bS)-9-phenyl-8-tosyl-8H,11bH-8a,11a-ethanofuro[3',2':3,4]pyrazolo[1,5-c]quinazoli n-10-yl)phenyl)ethan-1-one (5u) 


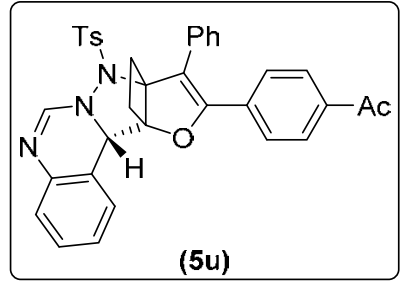

According to general procedure (GP2-A) with $\mathrm{PPh}_{3} \mathrm{AuCl}$ (1.2 mg, 2.4 $\mu \mathrm{mol}, 1 \mathrm{~mol} \%), \mathrm{AgSbF}_{6}(6.3 \mathrm{mg}, 18 \mu \mathrm{mol}, 10 \mathrm{~mol} \%)$, quinazolin-3-ium-3-yl(tosyl)amide 3a (65.1 mg, $0.218 \mathrm{mmol}, 1.1$ equiv), 1-(4-acetylphenyl)-3-cyclopropylidene-2-phenylprop-2-en-1-one $\mathbf{1 u}$ (56.7

mg, 0.197 mmol, 1.0 equiv), $100 \mathrm{mg} 4 \AA \mathrm{MS}, 2.0 \mathrm{~mL}$ of dry EA for 12 hours reaction. Purification by flash column chromatography provided $5 \mathbf{u}$ as a white solid (89.0 $\mathrm{mg}, 77 \%$ ); $d r=11: 1$ (ratio was determined by ${ }^{1} \mathrm{H}$ NMR analysis of the crude product); M.p. $188-189{ }^{\circ} \mathrm{C}$ (Petroleum ether/EtOAc); $\mathrm{R}_{f}=0.23$ (Petroleum ether/EtOAc $\left.=3 / 1\right) ;{ }^{1} \mathrm{H}$ NMR (400 MHz, $\left.\mathrm{CDCl}_{3}\right): \delta 7.72(\mathrm{~d}, J=8.4 \mathrm{~Hz}, 2 \mathrm{H})$, 7.56 (d, $J=8.0$ Hz, 2H), 7.45-7.30 (m, 6H), 7.28-7.15 (m, 7H), 6.41 (s, 1H), 5.16 (s, 1H), 3.38-3.25 (m, 1H), 3.09-2.97 (m, 1H), 2.80-2.67 (m, 1H), 2.58-2.44 (m, 4H), 2.37 (s, 3H); ${ }^{13} \mathrm{C}$ NMR (100 MHz, $\left.\mathrm{CDCl}_{3}\right): \delta 197.2,155.8,150.7,144.9,139.5,137.3,134.7,134.6,132.2,129.6,129.4,129.0,128.6$, 128.0, 127.9, 127.7, 126.4, 126.25, 126.20, 120.3, 113.7, 94.8, 80.7, 65.7, 26.5, 25.42, 25.37, 21.5; HRMS (ESI) m/z: [M + H] Calcd for $\mathrm{C}_{35} \mathrm{H}_{30} \mathrm{~N}_{3} \mathrm{O}_{4} \mathrm{~S}$ 588.1952; Found 588.1967.

(11aS,11bS)-10-isopropyl-9-phenyl-8-tosyl-8H,11bH-8a,11a-ethanofuro[3',2':3,4]pyrazolo[1,5-c]q uinazoline (5v)

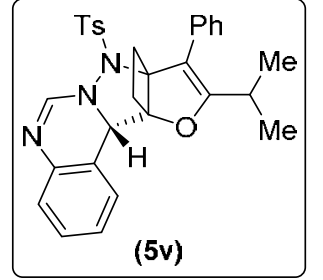

According to general procedure (GP2-A) with $\mathrm{PPh}_{3} \mathrm{AuCl}$ (1.2 mg, $2.4 \mu \mathrm{mol}, 1$ mol \%), $\operatorname{AgSbF}_{6} \quad(7.2 \quad \mathrm{mg}, 21 \mu \mathrm{mol}, 10 \mathrm{~mol} \%)$, quinazolin-3-ium-3-yl(tosyl)amide 3a (66.0 mg, $0.218 \mathrm{mmol}, 1.1$ equiv), 1-cyclopropylidene-4-methyl-2-phenylpent-1-en-3-one $\quad \mathbf{1 k} \quad$ (42.6 mg, 0.201

mmol, 1.0 equiv), $100 \mathrm{mg} 4 \AA \mathrm{MS}, 2.0 \mathrm{~mL}$ of dry EA for 12 hours reaction. Purification by flash column chromatography provided $5 \mathbf{v}$ as a white solid (61.6 $\mathrm{mg}, 60 \%$ ); $d r=3: 1$ (ratio was determined by ${ }^{1} \mathrm{H}$ NMR analysis of the crude product); M.p. 137-138 ${ }^{\circ} \mathrm{C}$ (Petroleum ether/EtOAc); $\mathrm{R}_{f}=0.57$ (Petroleum ether/EtOAc $\left.=3 / 1\right) ;{ }^{1} \mathrm{H}$ NMR (400 MHz, $\left.\mathrm{CDCl}_{3}\right): \delta 7.68(\mathrm{~d}, J=8.0 \mathrm{~Hz}, 2 \mathrm{H})$, 7.55-7.48 (m, 2H), 7.42 (t, $J=7.8$ Hz, 2H), 7.36-7.25 (m, 4H), 7.21-7.11 (m, 3H), 6.40 (s, 1H), 5.08 (s, 1H), 3.25-3.13 (m, 1H), 2.84-2.74 (m, 2H), 2.65-2.56 (m, 1H), 2.40 (s, 3H), 2.33-2.22 (m, 1H), $1.00(\mathrm{~d}, J=6.8 \mathrm{~Hz}, 3 \mathrm{H}), 0.84$ (d, $J=6.8 \mathrm{~Hz}, 3 \mathrm{H}) ;{ }^{13} \mathrm{C}$ NMR $\left(100 \mathrm{MHz}, \mathrm{CDCl}_{3}\right): \delta 166.1,150.8$, 144.8, 139.6, 134.9, 132.9, 129.7, 129.1, 128.6, 128.4, 128.3, 126.7, 126.2, 126.1, 120.7, 109.0, 94.7, 80.1, 65.3, 26.6, 25.2, 25.0, 21.6, 19.9, 18.4; HRMS (ESI) m/z: $[\mathrm{M}+\mathrm{H}]^{+}$Calcd for $\mathrm{C}_{30} \mathrm{H}_{30} \mathrm{~N}_{3} \mathrm{O}_{3} \mathrm{~S}$ 512.2002; Found 512.2012.

(11aS,11bS)-9-phenyl-10-(thiophen-2-yl)-8-tosyl-8H,11bH-8a,11a-ethanofuro[3',2':3,4]pyrazolo[1, 


\section{5-clquinazoline (5w)}

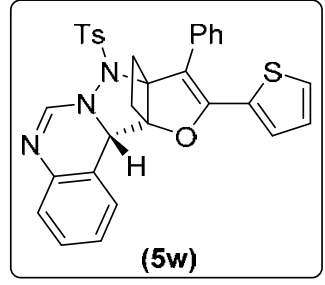

According to general procedure (GP2-A) with $\mathrm{PPh}_{3} \mathrm{AuCl}(1.1 \mathrm{mg}, 2.2 \mu \mathrm{mol}, 1$ mol \%), AgSbF$_{6} \quad(7.3 \quad \mathrm{mg}, 21 \mu \mathrm{mol}, 10$ mol \%), quinazolin-3-ium-3-yl(tosyl)amide 3a (66.2 mg, $0.221 \mathrm{mmol}, 1.1$ equiv), 3-cyclopropylidene-2-phenyl-1-(thiophen-2-yl)prop-2-en-1-one 11 (50.2 mg, $0.199 \mathrm{mmol}, 1.0$ equiv), $100 \mathrm{mg} 4 \AA \mathrm{MS}, 2.0 \mathrm{~mL}$ of dry EA for 12 hours reaction. Purification by flash column chromatography provided $5 \mathrm{w}$ as a white solid (86.2 $\mathrm{mg}, 79 \%$ ); $d r=16$ : 1 (ratio was determined by ${ }^{1} \mathrm{H}$ NMR analysis of the crude product); M.p. 202-203 ${ }^{\circ} \mathrm{C}$ (Petroleum ether/EtOAc); $\mathrm{R}_{f}=0.37$ (Petroleum ether/EtOAc $\left.=3 / 1\right) ;{ }^{1} \mathrm{H}$ NMR (400 MHz, $\left.\mathrm{CDCl}_{3}\right): \delta 7.65(\mathrm{~d}, J=8.0 \mathrm{~Hz}, 2 \mathrm{H})$, 7.53-7.47 (m, 2H), 7.46-7.36 (m, 3H), 7.32-7.23 (m, 3H), 7.22-7.15 (m, 3H), 7.12 (d, $J=4.8 \mathrm{~Hz}, 1 \mathrm{H})$, 6.93-6.88 (m, 1H), 6.81-6.76 (m, 1H), 6.46 (s, 1H), 5.13 (s, 1H), 3.24-3.14 (m, 1H), 2.95-2.84 (m, 1H), 2.73-2.64 (m, 1H), 2.53-2.42 (m, 1H), 2.39 (s, 3H); ${ }^{13} \mathrm{C}$ NMR (100 MHz, $\left.\mathrm{CDCl}_{3}\right): \delta 151.8$, 150.6, 144.9, 139.6, 134.8, 132.0, 131.6, 129.7, 129.3, 128.6, 128.0, 127.9, 127.5, 126.8, 126.3, 126.23, 126.17, 120.4, 110.7, 95.1, 80.7, 65.6, 25.2, 24.9, 21.6; HRMS (ESI) m/z: [M + H] Calcd for $\mathrm{C}_{31} \mathrm{H}_{26} \mathrm{~N}_{3} \mathrm{O}_{3} \mathrm{~S}_{2}$ 552.1410; Found 552.1417.

(11aS,11bS)-10-(furan-2-yl)-9-phenyl-8-tosyl-8H,11bH-8a,11a-ethanofuro[3',2':3,4]pyrazolo[1,5-c

\section{Jquinazoline $(5 x)$}

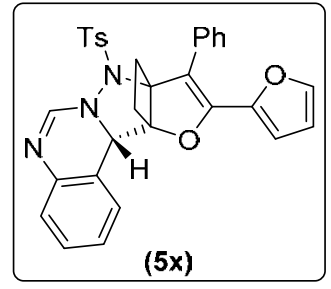

According to general procedure (GP2-A) with $\mathrm{PPh}_{3} \mathrm{AuCl}(0.9 \mathrm{mg}, 1.8 \mu \mathrm{mol}, 1$ mol \%), $\mathrm{AgSbF}_{6} \quad(7.3 \quad \mathrm{mg}, \quad 21 \mu \mathrm{mol}, 10 \quad \mathrm{~mol} \%)$, quinazolin-3-ium-3-yl(tosyl)amide 3a (66.1 mg, $0.221 \mathrm{mmol}, 1.1$ equiv), 3-cyclopropylidene-1-(furan-2-yl)-2-phenylprop-2-en-1-one $\mathbf{1 m} \quad$ (47.7 mg, $0.202 \mathrm{mmol}, 1.0$ equiv), $100 \mathrm{mg} 4 \AA \mathrm{MS}, 2.0 \mathrm{~mL}$ of dry EA for 12 hours reaction. Purification by flash column chromatography provided $\mathbf{5 x}$ as a white solid (83.2 $\mathrm{mg}, 77 \%$ ); $d r=12: 1$ (ratio was determined by ${ }^{1} \mathrm{H}$ NMR analysis of the crude product); M.p. 202-203 ${ }^{\circ} \mathrm{C}$ (Petroleum ether/EtOAc); $\mathrm{R}_{f}=0.35$ (Petroleum ether/EtOAc $\left.=3 / 1\right) ;{ }^{1} \mathrm{H}$ NMR $\left(400 \mathrm{MHz}, \mathrm{CDCl}_{3}\right): \delta 7.64(\mathrm{~d}, J=8.4 \mathrm{~Hz}, 2 \mathrm{H})$, 7.57 (d, $J=7.6 \mathrm{~Hz}, 2 \mathrm{H})$, 7.45-7.32 (m, 3H), 7.30-7.12 (m, 7H), 6.42 (s, 1H), 6.25 (s, 2H), 5.15 (s, 1H), 3.30-3.19 (m, 1H), 2.95-2.86 (m, 1H), 2.72-2.64 (m, 1H), 2.52-2.41 (m, 1H), 2.38 (s, 3H); ${ }^{13} \mathrm{C}$ NMR (100 MHz, $\left.\mathrm{CDCl}_{3}\right): \delta 150.5,147.9,144.9,144.7,143.3,139.5,134.7,131.7,129.6,129.2$, 129.1, 128.6, 128.1, 127.5, 126.3, 126.21, 126.17, 120.2, 112.3, 111.2, 111.1, 95.2, 80.4, 65.5, 25.12, 25.06, 21.5; HRMS (ESI) m/z: [M + H] $]^{+}$Calcd for $\mathrm{C}_{31} \mathrm{H}_{26} \mathrm{~N}_{3} \mathrm{O}_{4} \mathrm{~S}$ 536.1639; Found 536.1642. 


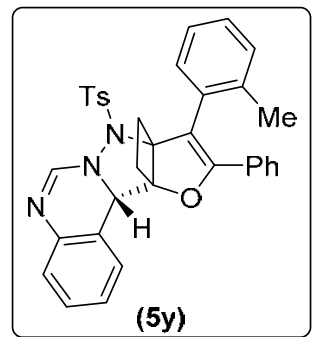

According to general procedure (GP2-A) with $\mathrm{PPh}_{3} \mathrm{AuCl}(1.1 \mathrm{mg}, 2.2 \mu \mathrm{mol}, 1$ mol \%), $\operatorname{AgSbF}_{6} \quad(7.2 \quad \mathrm{mg}, 21 \mu \mathrm{mol}, 10 \mathrm{~mol} \%)$, quinazolin-3-ium-3-yl(tosyl)amide 3a (65.4 mg, 0.219 mmol, 1.1 equiv), 3-cyclopropylidene-1-phenyl-2-(o-tolyl)prop-2-en-1-one $\quad 1 v$ (52.0 mg, 0.200 mmol, 1.0 equiv), $100 \mathrm{mg} 4 \AA \mathrm{MS}, 2.0 \mathrm{~mL}$ of dry EA for 12 hours reaction. Purification by flash column chromatography provided 5y as a white solid (81.4 mg, 73\%); $d r=10$ : 1 (ratio was determined by ${ }^{1} \mathrm{H}$ NMR analysis of the crude product); M.p. 203-204 ${ }^{\circ} \mathrm{C}$ (Petroleum ether/EtOAc); $\mathrm{R}_{f}=0.34$ (Petroleum ether/EtOAc = 3/1); ${ }^{1} \mathrm{H}$ NMR $\left(400 \mathrm{MHz}, \mathrm{CDCl}_{3}\right): \delta 7.70(\mathrm{~s}, 2 \mathrm{H})$, 7.41 (s, 1H), 7.35-7.26 (m, 6H), 7.25-7.18 (m, 3H), 7.16-7.10 (m, 1H), 7.09-6.95 (m, 4H), 6.53 (s, 1H), 5.16 (s, 1H), 3.20-3.01 (m, 1H), 3.00-2.80 (m, 1H), 2.74-2.64 (m, 1H), 2.55-2.45 (m, 1H), 2.41 (s, 3H), 2.03 (s, 3H); ${ }^{13} \mathrm{C}$ NMR (100 MHz, $\left.\mathrm{CDCl}_{3}\right): \delta$ 156.2, 150.6, 144.9, 139.5, 137.4, 134.8, 131.9, 130.6, 130.5, 130.2, 129.7, 129.3, 129.1, 128.7, 128.1, 128.0, 126.6, 126.3, 126.12, 126.06, 120.6, 110.1, 94.6, 81.2, 65.4, 25.0, 24.9, 21.6, 20.1; HRMS (ESI) m/z: $[\mathrm{M}+\mathrm{H}]^{+}$Calcd for $\mathrm{C}_{34} \mathrm{H}_{30} \mathrm{~N}_{3} \mathrm{O}_{3} \mathrm{~S}$ 560.2002; Found 560.2015.

\section{(11aS,11bS)-9-(3-fluorophenyl)-10-phenyl-8-tosyl-8H,11bH-8a,11a-ethanofuro[3',2':3,4]pyrazolo[}

\section{1,5-c]quinazoline (5z)}

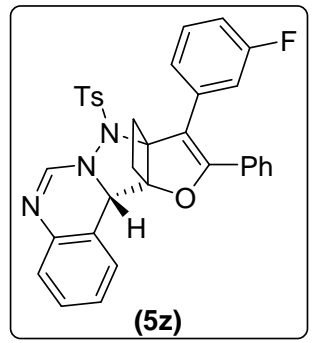

According to general procedure (GP2-A) with $\mathrm{PPh}_{3} \mathrm{AuCl}(1.0 \mathrm{mg}, 2.0 \mu \mathrm{mol}, 1$ mol \%), $\operatorname{AgSbF}_{6} \quad(6.8 \quad \mathrm{mg}, 20 \mu \mathrm{mol}, 10 \quad \mathrm{~mol} \%)$, quinazolin-3-ium-3-yl(tosyl)amide 3a (65.9 mg, $0.220 \mathrm{mmol}, 1.1$ equiv), 3-cyclopropylidene-2-(3-fluorophenyl)-1-phenylprop-2-en-1-one 1n (53.0 mg, $0.201 \mathrm{mmol}, 1.0$ equiv), $100 \mathrm{mg} 4 \AA \mathrm{MS}, 2.0 \mathrm{~mL}$ of dry EA for 3 hours reaction.

Purification by flash column chromatography provided $5 z$ as a white solid (70.1 mg, 62\%); $d r=9: 1$ (ratio was determined by ${ }^{1} \mathrm{H}$ NMR analysis of the crude product); M.p. $172-174{ }^{\circ} \mathrm{C}$ (Petroleum ether/EtOAc); $\mathrm{R}_{f}=0.48$ (Petroleum ether/EtOAc = 3/1); ${ }^{1} \mathrm{H}$ NMR $\left(400 \mathrm{MHz}, \mathrm{CDCl}_{3}\right): \delta 7.63(\mathrm{~d}, J=$ 8.0 Hz, 2H), 7.35-7.10 (m, 14H), 7.03-6.94 (m, 1H), 6.41 (s, 1H), 5.17 (s, 1H), 3.38-3.29 (m, 1H), 3.04-2.90 (m, 1H), 2.77-2.68 (m, 1H), 2.54-2.41 (m, 1H), 2.37 (s, 3H); $\left.{ }^{13} \mathrm{C} \mathrm{NMR} \mathrm{(100} \mathrm{MHz,} \mathrm{CDCl}_{3}\right)$ : $\delta 162.7$ (d, $J=243.9 \mathrm{~Hz}), 158.3,150.6$ (d, $J=3.3 \mathrm{~Hz}), 145.0,139.5,134.9$ (d, $J=8.3 \mathrm{~Hz}), 134.6$, $129.9(\mathrm{~d}, J=11.9 \mathrm{~Hz}), 129.8,129.7,129.4,128.6,128.3,127.9,126.4,126.3,126.2,124.5,120.3$, 
115.4 (d, $J=21.2 \mathrm{~Hz}$ ), 114.0 (d, $J=21.0 \mathrm{~Hz}$ ), 110.6, 94.8, 80.5, 65.6, 25.43, 25.35, 21.6; HRMS (ESI) $\mathrm{m} / \mathrm{z}:[\mathrm{M}+\mathrm{H}]^{+}$Calcd for $\mathrm{C}_{33} \mathrm{H}_{27} \mathrm{FN}_{3} \mathrm{O}_{3} \mathrm{~S}$ 564.1752; Found 564.1756.

(11aS,11bS)-10-phenyl-9-(p-tolyl)-8-tosyl-8H,11bH-8a,11a-ethanofuro[3',2':3,4]pyrazolo[1,5-c]qui nazoline (5aa)

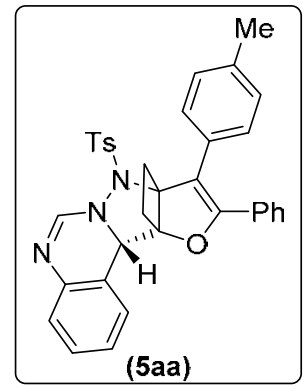

According to general procedure (GP2-A) with $\mathrm{PPh}_{3} \mathrm{AuCl}$ (1.1 mg, $2.2 \mu \mathrm{mol}, 1$ mol \%), $\operatorname{AgSbF}_{6} \quad(6.8 \quad \mathrm{mg}, 20 \mu \mathrm{mol}, 10 \quad \mathrm{~mol} \%)$, quinazolin-3-ium-3-yl(tosyl)amide $3 a$ (64.9 mg, $0.217 \mathrm{mmol}, 1.1$ equiv), 3-cyclopropylidene-1-phenyl-2-(p-tolyl)prop-2-en-1-one 10 (52.2 mg, 0.201 mmol, 1.0 equiv), $100 \mathrm{mg} 4 \AA \mathrm{MS}, 2.0 \mathrm{~mL}$ of dry EA for 12 hours reaction.

Purification by flash column chromatography provided 5aa as a white solid (90.2 mg, 80\%); $d r=9$ : 1 (ratio was determined by ${ }^{1} \mathrm{H}$ NMR analysis of the crude product); M.p. $162-163{ }^{\circ} \mathrm{C}$ (Petroleum ether/EtOAc); $\mathrm{R}_{f}=0.40$ (Petroleum ether/EtOAc = 3/1); ${ }^{1} \mathrm{H}$ NMR $\left(400 \mathrm{MHz}, \mathrm{CDCl}_{3}\right): \delta 7.59(\mathrm{~d}, J=$ 8.4 Hz, 2H), 7.35-7.26 (m, 3H), 7.25-7.10 (m, 12H), 6.42 (s, 1H), 5.14 (s, 1H), 3.34-3.22 (m, 1H), 3.05-2.95 (m, 1H), 2.74-2.64 (m, 1H), 2.53-2.42 (m, 1H), 2.37 (s, 6H); $\left.{ }^{13} \mathrm{C} \mathrm{NMR} \mathrm{(100} \mathrm{MHz,} \mathrm{CDCl}_{3}\right)$ : $\delta 156.8,150.9,144.8,139.6,136.9,134.9,130.5,129.6,129.3,129.1,128.7,128.6,128.1,127.8$, 126.3, 126.2, 120.5, 111.5, 94.4, 80.8, 65.7, 25.5, 25.4, 21.5, 21.2; HRMS (ESI) m/z: [M + H] Calcd for $\mathrm{C}_{34} \mathrm{H}_{30} \mathrm{~N}_{3} \mathrm{O}_{3} \mathrm{~S}$ 560.2002; Found 560.2010.

(11aS,11bS)-9-(4-methoxyphenyl)-10-phenyl-8-tosyl-8H,11bH-8a,11a-ethanofuro[3',2':3,4]pyrazol o[1,5-c]quinazoline (5ab)

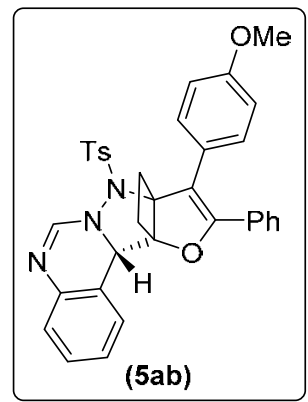

According to general procedure (GP2-A) with $\mathrm{PPh}_{3} \mathrm{AuCl}(1.0 \mathrm{mg}, 2.0 \mu \mathrm{mol}, 1$ mol \%), $\operatorname{AgSbF}_{6} \quad(7.0 \quad \mathrm{mg}, 20 \mu \mathrm{mol}, 10 \mathrm{~mol} \%)$, quinazolin-3-ium-3-yl(tosyl)amide 3a (65.4 mg, $0.219 \mathrm{mmol}, 1.1$ equiv), 3-cyclopropylidene-2-(4-methoxyphenyl)-1-phenylprop-2-en-1-one 1q (55.1 mg, $0.200 \mathrm{mmol}, 1.0$ equiv), $100 \mathrm{mg} 4 \AA \mathrm{MS}, 2.0 \mathrm{~mL}$ of dry EA for 12 hours reaction.

Purification by flash column chromatography provided 5ab as a white solid (86.1 mg, 75\%); $d r=9$ : 1 (ratio was determined by ${ }^{1} \mathrm{H}$ NMR analysis of the crude product); M.p. 192-193 ${ }^{\circ} \mathrm{C}$ (Petroleum ether/EtOAc); $\mathrm{R}_{f}=0.26$ (Petroleum ether/EtOAc = 3/1); ${ }^{1} \mathrm{H}$ NMR $\left(400 \mathrm{MHz}, \mathrm{CDCl}_{3}\right): \delta 7.60(\mathrm{~d}, J=$ 7.6 Hz, 2H), 7.41-7.08 (m, 13H), 6.88 (d, $J=7.6 \mathrm{~Hz}, 2 \mathrm{H}), 6.42$ (s, 1H), 5.14 (s, 1H), 3.82 (s, 3H), 3.35-3.21 (m, 1H), 3.06-2.93 (m, 1H), 2.74-2.64 (m, 1H), 2.55-2.41 (m, 1H), $2.36(\mathrm{~s}, 3 \mathrm{H}) ;{ }^{13} \mathrm{C}$ NMR (100 MHz, $\left.\mathrm{CDCl}_{3}\right): \delta$ 158.8, 156.3, 150.8, 144.8, 139.6, 134.8, 130.5, 130.0, 129.6, 129.23, 129.21, 
128.6, 128.0, 127.7, 126.3, 126.2, 124.8, 120.5, 113.8, 111.2, 94.3, 80.7, 65.7, 55.1, 25.4, 25.3, 21.5; HRMS (ESI) m/z: [M + H] $]^{+}$Calcd for $\mathrm{C}_{34} \mathrm{H}_{30} \mathrm{~N}_{3} \mathrm{O}_{4} \mathrm{~S}$ 576.1952; Found 576.1956.

1-(4-((11aS,11bS)-10-phenyl-8-tosyl-8H,11bH-8a,11a-ethanofuro[3',2':3,4]pyrazolo[1,5-c]quinazol in-9-yl)phenyl)ethan-1-one (5ac)

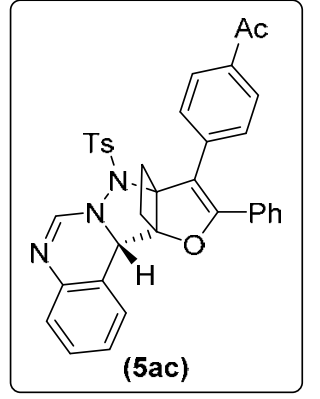

According to general procedure (GP2-A) with $\mathrm{PPh}_{3} \mathrm{AuCl}(1.2 \mathrm{mg}, 2.4 \mu \mathrm{mol}, 1$ mol \%), $\operatorname{AgSbF}_{6} \quad(6.7 \quad \mathrm{mg}, 19 \mu \mathrm{mol}, 10 \quad \mathrm{~mol} \%)$, quinazolin-3-ium-3-yl(tosyl)amide 3a (64.9 mg, $0.217 \mathrm{mmol}, 1.1$ equiv), 2-(4-acetylphenyl)-3-cyclopropylidene-1-phenylprop-2-en-1-one 1w (57.9 mg, $0.201 \mathrm{mmol}, 1.0$ equiv), $100 \mathrm{mg} 4 \AA \mathrm{MS}, 2.0 \mathrm{~mL}$ of dry EA for 12 hours reaction. Purification by flash column chromatography provided 5ac as a white solid (81.4 mg, 69\%); $d r=9: 1$ (ratio was determined by ${ }^{1} \mathrm{H}$ NMR analysis of the crude product); M.p. 188-189 ${ }^{\circ} \mathrm{C}$ (Petroleum ether/EtOAc); $\mathrm{R}_{f}=0.16$ (Petroleum ether/EtOAc = 3/1); ${ }^{1} \mathrm{H}$ NMR $\left(400 \mathrm{MHz}, \mathrm{CDCl}_{3}\right)$ : $\delta 7.91(\mathrm{~d}, J=6.8 \mathrm{~Hz}, 2 \mathrm{H}), 7.68-7.49(\mathrm{~m}, 4 \mathrm{H}), 7.38-7.10(\mathrm{~m}, 11 \mathrm{H}), 6.40(\mathrm{~s}, 1 \mathrm{H}), 5.19(\mathrm{~s}, 1 \mathrm{H})$, 3.44-3.30 (m, 1H), 3.10-2.95 (m, 1H), 2.80-2.68 (m, 1H), 2.61 (s, 3H), 2.55-2.42 (m, 1H), 2.37 (s, 3H); ${ }^{13} \mathrm{C}$ NMR (100 MHz, $\left.\mathrm{CDCl}_{3}\right): \delta$ 197.5, 159.5, 150.6, 145.0, 139.5, 137.9, 135.4, 134.5, 130.05, 129.98, 129.7, 129.4, 128.6, 128.4, 128.0, 126.5, 126.3, 126.2, 120.2, 110.9, 95.1, 80.3, 65.5, 26.5, 25.5, 25.4, 21.5; HRMS (ESI) m/z: [M + H] ${ }^{+}$Calcd for $\mathrm{C}_{35} \mathrm{H}_{30} \mathrm{~N}_{3} \mathrm{O}_{4} \mathrm{~S}$ 588.1952; Found 588.1953.

(11aS,11bS)-9-(4-(tert-butyl)phenyl)-10-phenyl-8-tosyl-8H,11bH-8a,11a-ethanofuro[3',2':3,4]pyra

\section{zolo[1,5-c]quinazoline (5ad)}

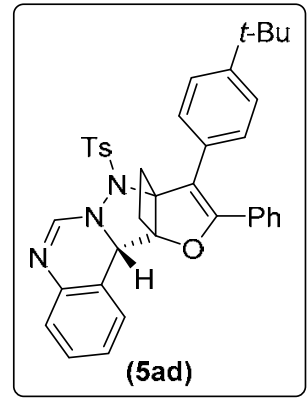

According to general procedure (GP2-A) with $\mathrm{PPh}_{3} \mathrm{AuCl}(0.9 \mathrm{mg}, 1.8 \mu \mathrm{mol}, 1$ mol \%), AgSbF$_{6} \quad(7.1 \quad \mathrm{mg}, \quad 21 \mu \mathrm{mol}, 10 \quad \mathrm{~mol} \%)$, quinazolin-3-ium-3-yl(tosyl)amide 3a (65.3 mg, $0.218 \mathrm{mmol}, 1.1$ equiv), 2-(4-(tert-butyl)phenyl)-3-cyclopropylidene-1-phenylprop-2-en-1-one 1p (59.3 mg, 0.196 mmol, 1.0 equiv), $100 \mathrm{mg} 4 \AA \mathrm{MS}, 2.0 \mathrm{~mL}$ of dry EA for 12 hours reaction. Purification by flash column chromatography provided 5ad as a white solid (92.0 mg, 78\%); $d r=9: 1$ (ratio was determined by ${ }^{1} \mathrm{H}$ NMR analysis of the crude product); M.p. 190-191 ${ }^{\circ} \mathrm{C}$ (Petroleum ether/EtOAc); $\mathrm{R}_{f}=0.50$ (Petroleum ether/EtOAc $\left.=3 / 1\right) ;{ }^{1} \mathrm{H}$ NMR $\left(400 \mathrm{MHz}, \mathrm{CDCl}_{3}\right): \delta$ 7.55 (d, $J=8.0$ Hz, 2H), 7.41-7.30 (m, 4H), 7.29-7.10 (m, 11H), 6.38 (s, 1H), 5.15 (s, 1H), 3.38-3.26 (m, 1H), 3.08-2.97 (m, 1H), 2.75-2.64 (m, 1H), 2.52-2.41 (m, 1H), 2.35 (s, 3H), 1.35 (s, 9H); ${ }^{13} \mathrm{C}$ NMR (100 MHz, $\left.\mathrm{CDCl}_{3}\right): \delta$ 157.1, 150.8, 149.9, 144.7, 139.6, 135.0, 130.6, 129.5, 129.3, 129.2, 
128.5, 128.2, 128.1, 127.9, 126.3, 126.18, 126.15 125.2, 120.5, 111.4, 94.4, 80.9, 65.8, 34.5, 31.3, 25.6, 25.4, 21.5; HRMS (ESI) m/z: [M + H] $]^{+}$Calcd for $\mathrm{C}_{37} \mathrm{H}_{36} \mathrm{~N}_{3} \mathrm{O}_{3} \mathrm{~S}$ 602.2472; Found 602.2478.

(8bR,13bR,13cS)-15,16-diphenyl-8-tosyl-8b,10,11,12,13,13a-hexahydro-8H,9H,13cH-13b,8a-(epox yetheno)cyclohepta[3',4']cyclobuta[1',2':3,4]pyrazolo[1,5-c]quinazoline (5ae)

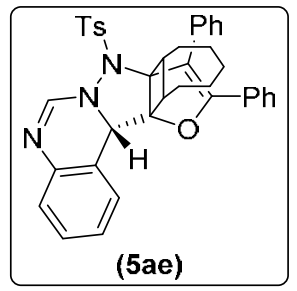

According to general procedure (GP2-A) with $\mathrm{PPh}_{3} \mathrm{AuCl}$ (0.61 mg, $0.93 \mu \mathrm{mol}, 1$ mol \%), $\operatorname{AgSbF}_{6} \quad(3.2 \quad \mathrm{mg}, \quad 9.3 \mu \mathrm{mol}, 10 \mathrm{~mol} \%)$, quinazolin-3-ium-3-yl(tosyl)amide 3a (21.0 mg, $0.0702 \mathrm{mmol}, 1.1$ equiv), 3-cycloheptylidene-1,2-diphenylprop-2-en-1-one 1x (21.8 mg, 0.0694 mmol, 1.0

equiv), $50 \mathrm{mg} 4 \AA \mathrm{MS}, 1.0 \mathrm{~mL}$ of dry THF for 12 hours reaction. Purification by flash column chromatography provided 5ae as a white solid (32.8 mg, 71\%); $d r=8: 1$ (ratio was determined by ${ }^{1} \mathrm{H}$ NMR analysis of the crude product); M.p. 267-269 ${ }^{\circ} \mathrm{C}$ (Petroleum ether/EtOAc); $\mathrm{R}_{f}=0.26$ $\left(\right.$ Petroleum ether/EtOAc = 5/1); ${ }^{1} \mathrm{H}$ NMR $\left(400 \mathrm{MHz}_{\mathrm{CDCl}}\right): \delta 7.67$ (d, $\left.J=8.0 \mathrm{~Hz}, 2 \mathrm{H}\right), 7.46$ (d, $J=$ 7.2 Hz, 2H), 7.30-7.23 (m, 6H), 7.20-7.11 (m, 8H), 6.36 (s, 1H), 5.15 (s, 1H), 3.75-3.66 (m, 1H), 3.02-2.93 (m, 1H), 2.39 (s, 3H), 2.26-2.18 (m, 1H), 2.01-1.80 (m, 4H), 1.74-1.61 (m, 2H), 1.23-1.08 (m, 3H); ${ }^{13} \mathrm{C}$ NMR (100 MHz, $\left.\mathrm{CDCl}_{3}\right): \delta 160.1,150.8,144.8,139.6,135.1,133.5,130.7,129.7$, 129.5, 129.2, 128.7, 128.6, 128.31, 128.29, 128.1, 126.5, 126.3, 126.2, 126.1, 120.8, 110.8, 94.1, 83.8, 67.1, 42.1, 41.6, 31.7, 29.8, 28.2, 28.0, 25.8, 21.6; HRMS (ESI) m/z: $[\mathrm{M}+\mathrm{H}]^{+}$Calcd for $\mathrm{C}_{38} \mathrm{H}_{36} \mathrm{~N}_{3} \mathrm{O}_{3} \mathrm{~S}$ 614.2472; Found 614.2474.

(14bR,14cS)-16,17-diphenyl-8-tosyl-8b,9,10,11,12,13,14,14a-octahydro-8H,14cH-14b,8a-(epoxyeth eno)cycloocta[3',4']cyclobuta[1',2':3,4]pyrazolo[1,5-c]quinazoline (5af)

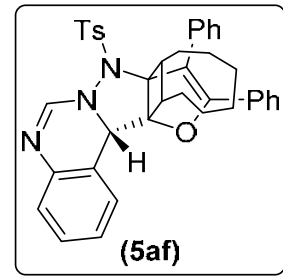

According to general procedure (GP2-A) with $\mathrm{PPh}_{3} \mathrm{AuCl}$ (1.7 mg, $3.3 \mu \mathrm{mol}, 1$ mol \%), AgSbF$_{6}$ (11 mg, $32 \mu \mathrm{mol}, 10$ mol \%), quinazolin-3-ium-3-yl(tosyl)amide 3a $\quad(98.7 \quad \mathrm{mg}, \quad 0.330 \quad \mathrm{mmol}, 1.1 \quad$ equiv $)$, 3-cyclooctylidene-1,2-diphenylprop-2-en-1-one 1y (99.0 mg, $0.302 \mathrm{mmol}, 1.0$ equiv), $100 \mathrm{mg} 4 \AA \mathrm{MS}, 2.0 \mathrm{~mL}$ of dry THF for 13 hours reaction. Purification by flash column chromatography provided 5af as a white solid (75.0 mg, 40\%); $d r=4: 1$ (ratio was determined by ${ }^{1} \mathrm{H}$ NMR analysis of the crude product); M.p. $266-268{ }^{\circ} \mathrm{C}$ (Petroleum ether/EtOAc); $\mathrm{R}_{f}=0.36$ (Petroleum ether/DCM = 1/2); ${ }^{1} \mathrm{H}$ NMR $\left(400 \mathrm{MHz}, \mathrm{CDCl}_{3}\right): \delta 7.86(\mathrm{~d}, J=8.0 \mathrm{~Hz}, 2 \mathrm{H}), 7.49(\mathrm{~d}, J=$ 7.6 Hz, 2H), 7.37-7.27 (m, 4H), 7.26-7.23 (m, 2H), 7.22-7.08 (m, 8H), 6.48 (s, 1H), 5.16 (s, 1H), 3.35-3.39 (m, 1H), 2.85-2.80 (m, 1H), 2.43 (s, 3H), 2.03-1.87 (m, 1H), 1.85-1.62 (m, 6H), 1.50-1.38 
(m, 2H), 1.24-1.04 (m, 3H); ${ }^{13} \mathrm{C}$ NMR (100 MHz, $\left.\mathrm{CDCl}_{3}\right): \delta 159.2,150.6,144.9,139.6,135.1,134.1$, 130.2, 129.8, 129.4, 129.12, 129.08, 128.6, 128.3, 128.2, 128.0, 126.8, 126.24, 126.22, 126.0, 120.9, 111.2, 95.3, 83.2, 66.7, 41.7, 40.9, 30.6, 28.4, 26.0, 25.2, 23.2, 22.8, 21.7; HRMS (ESI) m/z: [M + $\mathrm{H}]^{+}$Calcd for $\mathrm{C}_{39} \mathrm{H}_{38} \mathrm{~N}_{3} \mathrm{O}_{3} \mathrm{~S}$ 628.2628; Found 628.2629.

\section{Gram-scale synthesis of the products $4 a$ and $5 a$}

(a) Gram-scale synthesis of the product 4a
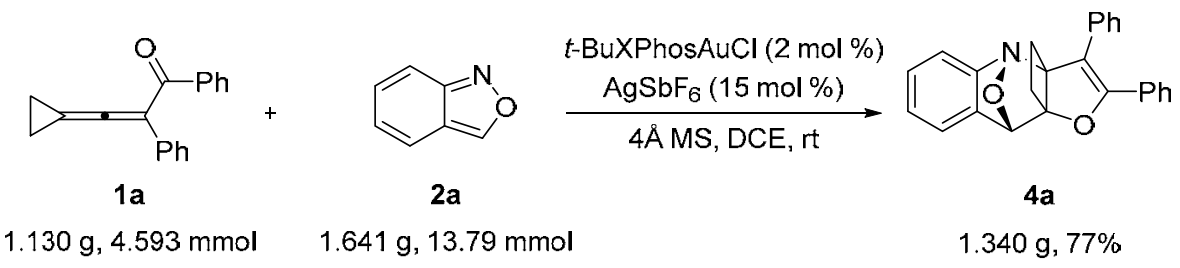

According to general procedure (GP1) with $t$-BuXPhosAuCl $(0.0604 \mathrm{~g}, 0.0920 \mathrm{mmol}, 2 \mathrm{~mol} \%)$, $\operatorname{AgSbF}_{6}(0.240$ g, 0.698 mmol, 15 mol \%), benzo[c]isoxazole 2a (1.641 g, 13.79 mmol, 3.0 equiv),

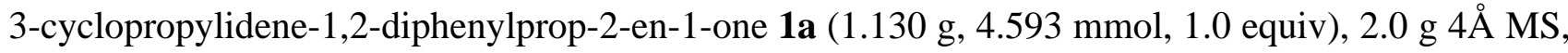
$45.0 \mathrm{~mL}$ of dry DCE for 4 hours reaction. Purification by flash column chromatography provided $\mathbf{4 a}$ as a white solid (1.340 g, 77\%).

(b) Gram-scale synthesis of the product 5a

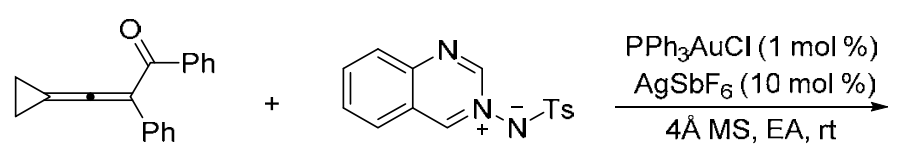

$1 \mathbf{a}$

$3 \mathbf{a}$

$0.6617 \mathrm{~g}, 2.057 \mathrm{mmol} \quad 0.8241 \mathrm{~g}, 2.756 \mathrm{mmol}$

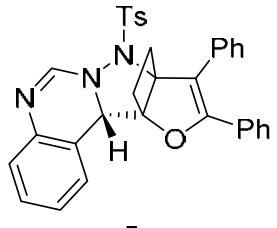

$5 \mathbf{a}$

$1.161 \mathrm{~g}, 85 \%$

According to general procedure (GP2-A) with $\mathrm{PPh}_{3} \mathrm{AuCl}(0.0123 \mathrm{~g}, 0.0249 \mathrm{mmol}, 1 \mathrm{~mol} \%)$, $\operatorname{AgSbF}_{6}$ (0.0875 g, 0.254 mmol, 10 mol \%), quinazolin-3-ium-3-yl(tosyl)amide 3a (0.8241 g, 2.756 mmol, 1.1 equiv), 3-cyclopropylidene-1,2-diphenylprop-2-en-1-one 1a (0.6617 g, 2.507 mmol, 1.0 equiv), $1.25 \mathrm{~g} 4 \AA \mathrm{MS}, 25.0 \mathrm{~mL}$ of dry EA for 12 hours reaction. Purification by flash column chromatography provided 5a as a white solid (1.161 g, 85\%); $d r=9: 1$ (ratio was determined by ${ }^{1} \mathrm{H}$ NMR analysis of the crude product).

\section{Transformation of the compounds 4a and compounds $5 a$}




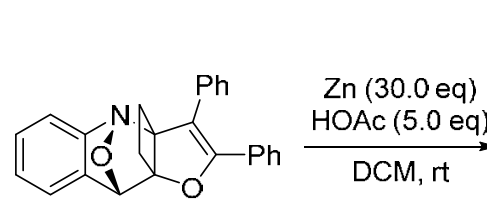

$4 \mathbf{a}$

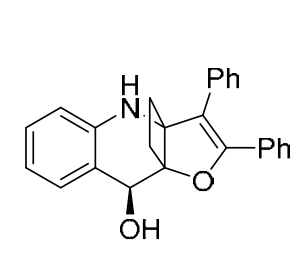

6

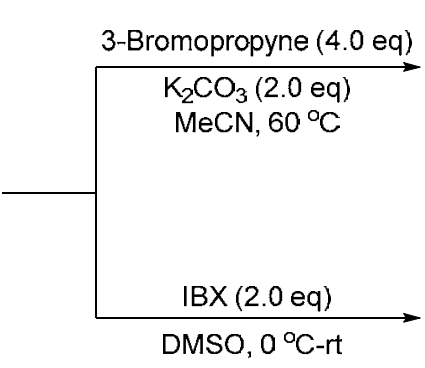

DMSO, $0^{\circ} \mathrm{C}-\mathrm{rt}$

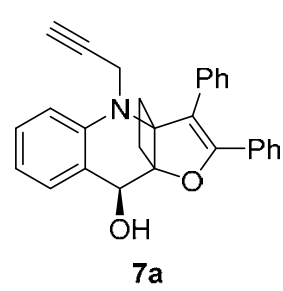

$7 a$

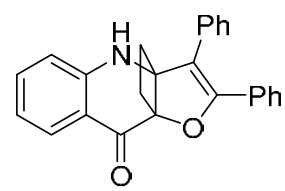

$7 \mathbf{b}$

(9S)-2,3-diphenyl-4,9-dihydro-3a,9a-ethanofuro[3,2-b]quinolin-9-ol (6)

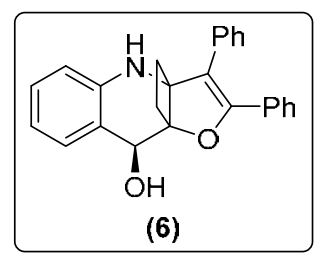

(6)

To a solution of 4a (35.9 mg, $0.0980 \mathrm{mmol}, 1.0 \mathrm{eq}$ ) in $2.0 \mathrm{~mL}$ DCM was added a Zn power (196.4 mg, 3.003 mmol, 30.0 eq) followed by HOAc (30.2 mg, 0.503 mmol, 5.0 eq) and the solution was stirred at room temperature for 3 hours under $\mathrm{N}_{2}$ atmosphere. The reaction was determined by TLC. After completion, reaction mixture was filtered over celite bed. Filtrate was washed with water and brine solution, then dried with $\mathrm{MgSO}_{4}$, concentrated under reduced pressure and purified by silica gel column chromatography to give product 6 as white solid (32.1 mg, 89\%). M.p. 138-139 ${ }^{\circ} \mathrm{C}$ (Petroleum ether/EtOAc); $\mathrm{R}_{f}=$ 0.18 (Petroleum ether/EtOAc $=10 / 1) ;{ }^{1} \mathrm{H}$ NMR (400 MHz, $\left.\mathrm{CDCl}_{3}\right): \delta 7.56(\mathrm{~d}, J=7.6 \mathrm{~Hz}, 1 \mathrm{H})$, 7.45-7.39 (m, 2H), 7.38-7.27 (m, 5H), 7.26-7.18 (m, 3H), 7.13 (t, $J=7.6 \mathrm{~Hz}, 1 \mathrm{H}), 6.92$ (t, $J=7.4 \mathrm{~Hz}$, 1H), 6.61 (d, $J=7.6 \mathrm{~Hz}, 1 \mathrm{H}), 4.93$ (d, $J=4.8 \mathrm{~Hz}, 1 \mathrm{H}), 3.97$ (s, 1H), 2.65-2.54 (m, 1H), 2.50 (d, $J=$ $5.6 \mathrm{~Hz}, 1 \mathrm{H}), 2.47-2.35$ (m, 1H), 2.33-2.22 (m, 1H); ${ }^{13} \mathrm{C}$ NMR (100 MHz, $\left.\mathrm{CDCl}_{3}\right): \delta 152.5,142.6$, 133.6, 131.1, 129.3, 129.0, 128.8, 128.3, 128.0, 127.6, 127.3, 126.1, 124.7, 119.9, 116.2, 115.3, 90.5, 69.7, 68.9, 31.3, 24.1; HRMS (ESI) m/z: [M + H] $]^{+}$Calcd for $\mathrm{C}_{25} \mathrm{H}_{22} \mathrm{NO}_{2}$ 368.1645; Found 368.1654.

\section{(9S)-2,3-diphenyl-4-(prop-2-yn-1-yl)-4,9-dihydro-3a,9a-ethanofuro[3,2-b]quinolin-9-ol (7a)}

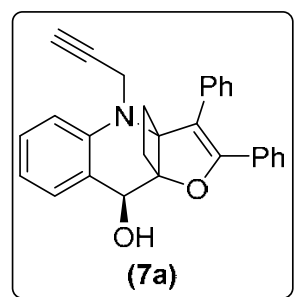

To a solution of 6 (55.9 mg, $0.152 \mathrm{mmol}, 1.0 \mathrm{eq})$ in $2.0 \mathrm{~mL} \mathrm{MeCN}$ was added $\mathrm{K}_{2} \mathrm{CO}_{3}$ (44.0 mg, $0.318 \mathrm{mmol}, 2.0 \mathrm{eq}$ ) and the solution was stirred at $60{ }^{\circ} \mathrm{C}$ in an oil bath. After being stirred for $5 \mathrm{~min}$, the 3-Bromopropyne $(74.0 \mathrm{mg}, 0.622$ mmol, 4.0 eq) was added. After being stirred for 4 days, the reaction was

determined by TLC. Purification by flash column chromatography provided 7a as a brown oil (38.0 mg, 62\%). $\mathrm{R}_{f}=0.3$ (Petroleum ether/DCM = 1/1); ${ }^{1} \mathrm{H}$ NMR (400 MHz, $\left.\mathrm{CDCl}_{3}\right): \delta 7.42$ (d, $J=6.4 \mathrm{~Hz}$, 1H), 7.35-7.29 (m, 3H), 7.27-7.22 (m, 3H), 7.21-7.11 (m, 5H), 6.93 (t, $J=7.2 \mathrm{~Hz}, 1 \mathrm{H}), 6.84$ (d, $J=$ 
$8.0 \mathrm{~Hz}, 1 \mathrm{H}), 4.79$ (d, $J=7.6 \mathrm{~Hz}, 1 \mathrm{H}), 3.46$ (s, 2H), 2.93-2.78 (m, 2H), 2.54 (d, $J=7.6 \mathrm{~Hz}, 1 \mathrm{H})$, 2.50-2.34 (m, 2H), 2.04 (t, $J=2.2 \mathrm{~Hz}, 1 \mathrm{H}) ;{ }^{13} \mathrm{C}$ NMR (100 MHz, $\left.\mathrm{CDCl}_{3}\right): \delta 153.5,143.1,135.3$, 130.9, 130.1, 129.2, 129.0, 128.9, 128.5, 128.3, 127.8, 127.5, 127.4, 120.3, 115.7, 115.4, 89.9, 80.3, 74.2, 71.7, 69.3, 35.1, 30.8, 25.1; HRMS (ESI) m/z: $[\mathrm{M}+\mathrm{H}]^{+}$Calcd for $\mathrm{C}_{28} \mathrm{H}_{24} \mathrm{NO}_{2}$ 406.1802; Found 406.1815.

\section{2,3-diphenyl-3a,9a-ethanofuro[3,2-b]quinolin-9(4H)-one (7b)}

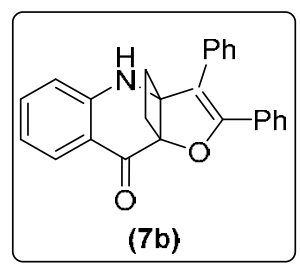

At $0{ }^{\circ} \mathrm{C}$ in an ice bath, to a solution of 6 (55.2 $\left.\mathrm{mg}, 0.150 \mathrm{mmol}, 1.0 \mathrm{eq}\right)$ in $2.0 \mathrm{~mL}$ DMSO was added IBX (84.7 mg, $0.303 \mathrm{mmol}, 2.0 \mathrm{eq})$. Then, the solution was stirred at room temperature for 1 hour under air. The reaction was determined by TLC. After completion, reaction mixture was washed with water and extracted with $10 \mathrm{~mL}$ EA. The combined organic extracts were washed with brine, then dried with $\mathrm{MgSO}_{4}$, concentrated under reduced pressure and purified by silica gel column chromatography to give product 7b as yellow solid (38.8 mg, 71\%). $\mathrm{R}_{f}=0.87$ (Petroleum ether/DCM = 1/2); ${ }^{1} \mathrm{H}$ NMR (400 MHz, $\mathrm{CDCl}_{3}$ ): $\delta 7.91$ (d, $\left.J=7.6 \mathrm{~Hz}, 1 \mathrm{H}\right), 7.45$ (d, $\left.J=6.8 \mathrm{~Hz}, 2 \mathrm{H}\right), 7.42-7.31$ (m, 5H), 7.29-7.18 (m, 4H), 6.75 (t, $J=7.6 \mathrm{~Hz}, 1 \mathrm{H}), 6.51$ (d, $J=8.4 \mathrm{~Hz}, 1 \mathrm{H}), 4.52$ (s, 1H), 2.81-2.66 (m, 3H), 2.54-2.42 (m, $1 \mathrm{H}) ;{ }^{13} \mathrm{C}$ NMR (100 MHz, $\left.\mathrm{CDCl}_{3}\right): \delta$ 189.2, 153.0, 148.8, 136.3, 132.9, 130.3, 129.4, 129.2, 129.1, 128.7, 128.0, 127.8, 127.7, 118.3, 116.4, 115.7, 115.0, 82.9, 68.4, 30.7, 30.3; HRMS (ESI) m/z: [M + $\mathrm{H}]^{+}$Calcd for $\mathrm{C}_{25} \mathrm{H}_{20} \mathrm{NO}_{2}$ 366.1489; Found 366.1502.

(b) Procedure for Synthesis of 8 and 9:

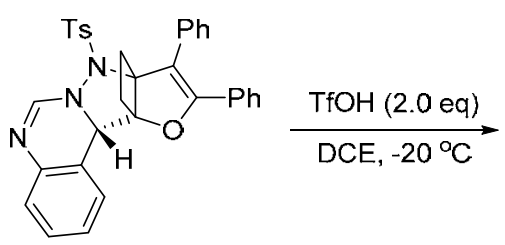

$5 \mathbf{a}$

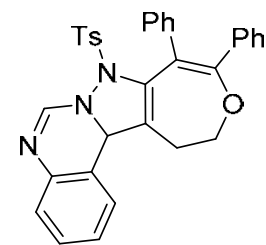

8

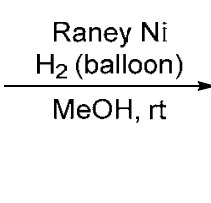

$\underset{\mathrm{MeOH}, \mathrm{rt}}{\stackrel{\mathrm{H}_{2} \text { (balloon) }}{\longrightarrow}}$

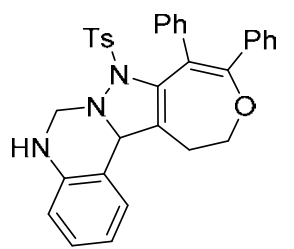

9

\section{9,10-diphenyl-8-tosyl-8,12,13,13b-tetrahydrooxepino[4',5':3,4]pyrazolo[1,5-c]quinazoline (8)}

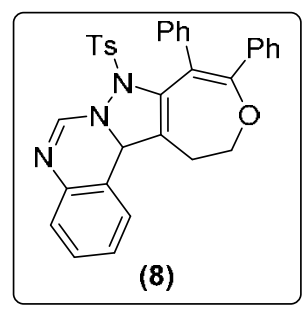

Under $\mathrm{N}_{2}$ atmosphere, to a solution of $5 \mathbf{a}(27.2 \mathrm{mg}, 49.9 \mu \mathrm{mol}, 1.0 \mathrm{eq})$ in $1.0 \mathrm{~mL}$ dry DCE was added TfOH (15.2 mg, $101 \mu \mathrm{mol}, 2.0$ equiv) at $-20{ }^{\circ} \mathrm{C}$. After stirring for $20 \mathrm{~min}$, the reaction was quenched with $\mathrm{Et}_{3} \mathrm{~N}$ and diluted with $\mathrm{CHCl}_{3}$.

The combined organic extracts were washed with $1 \mathrm{M}$ aqueous $\mathrm{HCl}$ followed by silica gel (hexane/EtOAc $=10 / 1)$ to give product 8 as a white solid $(25.1 \mathrm{mg}, 92 \%)$; M.p. $110-111{ }^{\circ} \mathrm{C}$ 
(Petroleum ether/EtOAc); $\mathrm{R}_{f}=0.14$ (Petroleum ether/EtOAc $=3 / 1$ ); ${ }^{1} \mathrm{H}$ NMR $\left(400 \mathrm{MHz}, \mathrm{CDCl}_{3}\right): \delta$ 7.75 (d, $J=8.4 \mathrm{~Hz}, 2 \mathrm{H}), 7.44-7.35$ (m, 3H), 7.33-7.27 (m, 3H), 7.25-7.09 (m, 10H), 6.94 (s, 1H), 5.76 (s, 1H), 3.98-3.91 (m, 2H), 2.81-2.69 (m, 1H), 2.68-2.58 (m, 1H), $2.40(\mathrm{~s}, 3 \mathrm{H}) ;{ }^{13} \mathrm{C}$ NMR (100 $\mathrm{MHz}_{\mathrm{CDCl}}$ ): $\delta 149.53,149.49,147.8,147.1,145.1,139.3,133.9,132.6,130.3,130.2,129.9,129.3$, 128.9, 128.2, 128.1, 127.7, 127.2, 126.1, 125.7, 125.2, 123.1, 122.3, 120.5, 56.7, 51.1, 21.6, 20.9; HRMS (ESI) m/z: [M + H] ${ }^{+}$Calcd for $\mathrm{C}_{33} \mathrm{H}_{28} \mathrm{~N}_{3} \mathrm{O}_{3} \mathrm{~S}$ 546.1846; Found 546.1855.

\section{9,10-diphenyl-8-tosyl-5,6,8,12,13,13b-hexahydrooxepino[4',5':3,4]pyrazolo[1,5-c]quinazoline (9)}

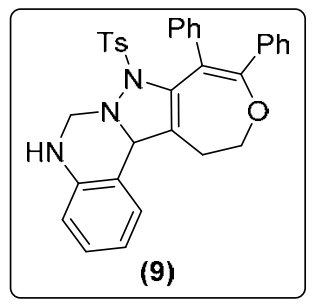

A solution of 8 (27.2 $\mathrm{mg}, 49.9 \mu \mathrm{mol})$ in $\mathrm{MeOH}(2.0 \mathrm{~mL})$ was hydrogenated at atmospheric pressure in the presence of Raney nickel $(\sim 1.0 \mathrm{~g})$ at room temperature. After stirring overnight, the reaction mixture was filtered through Celite and the solvent evaporated. Purification by flash column chromatography provided 9 as a white solid (13.1 mg, 48\%); M.p. 178-179 ${ }^{\circ} \mathrm{C}$ (Petroleum ether/EtOAc); $\mathrm{R}_{f}=0.43\left(\right.$ Petroleum ether/EtOAc = 3/1); ${ }^{1} \mathrm{H}$ NMR $\left(400 \mathrm{MHz}, \mathrm{CDCl}_{3}\right): \delta 7.72(\mathrm{~d}, J=$ 8.4 Hz, 2H), 7.42-7.30 (m, 3H), 7.25-7.08 (m, 10H), 6.89 (d, $J=7.6 \mathrm{~Hz}, 1 \mathrm{H}), 6.76$ (t, $J=7.4 \mathrm{~Hz}$, 1H), 6.66 (d, $J=8.0 \mathrm{~Hz}, 1 \mathrm{H}), 5.55$ (s, 1H), 4.52 (d, $J=10.8 \mathrm{~Hz}, 1 \mathrm{H}), 4.29$ (d, $J=10.8 \mathrm{~Hz}, 1 \mathrm{H})$, 4.21-4.09 (m, 1H), 3.80-3.70 (m, 1H), 2.68-2.58 (m, 1H), 2.42-2.33 (m, 1H), 2.19 (s, 3H), 1.62 (s, $1 \mathrm{H}) ;{ }^{13} \mathrm{C}$ NMR (100 MHz, $\left.\mathrm{CDCl}_{3}\right): \delta 148.1,147.5,143.0,141.9,135.9,133.0,130.6,129.7,129.1$, 128.7, 128.3, 128.2, 128.0, 127.7, 127.5, 127.1, 125.3, 123.4, 123.0, 120.2, 119.3, 116.6, 63.8, 59.4, 45.0, 23.4, 21.4; HRMS (ESI) m/z: [M + H] $]^{+}$Calcd for $\mathrm{C}_{33} \mathrm{H}_{30} \mathrm{~N}_{3} \mathrm{O}_{3} \mathrm{~S}$ 548.2002; Found 548.2011.

\section{The synthesis of compounds 11, 13, 15, 17, 19 and 21}

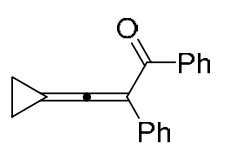

$1 \mathbf{a}$

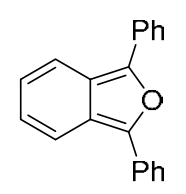

10

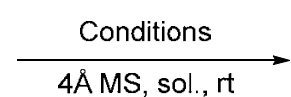

$t$-BuXPhosAuCl (2 mol \%)/AgSbF 6 (15 mol \%) in DCE, $\mathrm{PPh}_{3} \mathrm{AuCl}(1 \mathrm{~mol} \%) / \mathrm{AgSbF}_{6}(10 \mathrm{~mol} \%)$ in $\mathrm{EA}$, $\boldsymbol{t}$-BuXPhosAuCl (2 mol \%)/AgTFA (15 mol \%) in DCE, $\mathrm{PPh}_{3} \mathrm{AuCl}(1 \mathrm{~mol} \%) / \mathrm{AgTFA}(15 \mathrm{~mol} \%)$ in DCE, $\mathrm{PPh}_{3}$ AuOTf $(10 \%)$ in DCE,

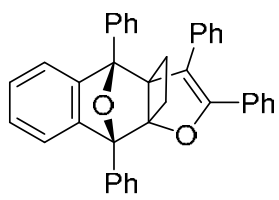

yield of 11

$0 \%$

$10 \%$

$62 \%$

$41 \%$ $0 \%$

(4R,9S)-2,3,4,9-tetraphenyl-4,9-dihydro-4,9-epoxy-3a,9a-ethanonaphtho[2,3-b]furan (11)

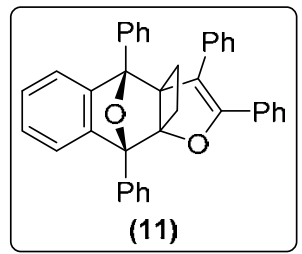

t-BuXPhosAuCl (1.4 mg, $2.1 \mu \mathrm{mol}, 2 \mathrm{~mol} \%$ ) and $\mathrm{CF}_{3} \mathrm{CO}_{2} \mathrm{Ag}(2.8 \mathrm{mg}, 13 \mu \mathrm{mol}$, $15 \mathrm{~mol} \%$ ) were dissolved in $2.0 \mathrm{~mL}$ of dry DCE in a dried vial under $\mathrm{N}_{2}$ 


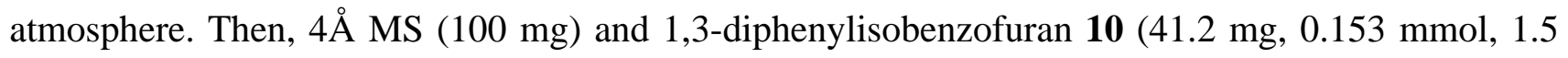
equiv) were added in this order and the reaction was stirred at room temperature. After being stirred for $5 \mathrm{~min}$, the 3-cyclopropylidene-1,2-diphenylprop-2-en-1-one 1a (24.8 $\mathrm{mg}, 0.101 \mathrm{mmol}, 1.0$ equiv) was added. The reaction was stirred at room temperature for 7 hours and determined by TLC. After completion, the solution was removed under reduced pressure, and purified by flash silica gel chromatography to afford product $\mathbf{1 1}$ as a white solid (32.1 mg, 62\%); endo : exo > 99 : 1 (ratio was determined by ${ }^{1} \mathrm{H}$ NMR analysis of the crude product); M.p. $221-224{ }^{\circ} \mathrm{C}$ (Petroleum ether/EtOAc); $\mathrm{R}_{f}=0.55$ (Petroleum ether/EtOAc $\left.=50 / 1\right) ;{ }^{1} \mathrm{H}$ NMR $\left(400 \mathrm{MHz}, \mathrm{CDCl}_{3}\right): \delta 7.77(\mathrm{~d}, J=7.6 \mathrm{~Hz}, 2 \mathrm{H})$, 7.55-7.40 (m, 3H), 7.29-7.03 (m, 14H), 7.00-6.98 (m, 5H), 2.68-2.58 (m, 2H), 2.49-2.28 (m, 2H); ${ }^{13} \mathrm{C}$ NMR (100 MHz, $\left.\mathrm{CDCl}_{3}\right): \delta 157.0,145.7,144.6,135.6,135.21,135.19,131.3,130.0,128.5,128.4$, 128.2, 128.1, 127.8, 127.7, 127.6, 127.5, 126.91, 126.88, 126.6, 121.6, 120.3, 114.1, 94.5, 92.1, 90.3, 67.5, 26.8, 26.1; HRMS (ESI) m/z: [M + H] $]^{+}$Calcd for $\mathrm{C}_{38} \mathrm{H}_{29} \mathrm{O}_{2}$ 517.2162; Found 517.2161.

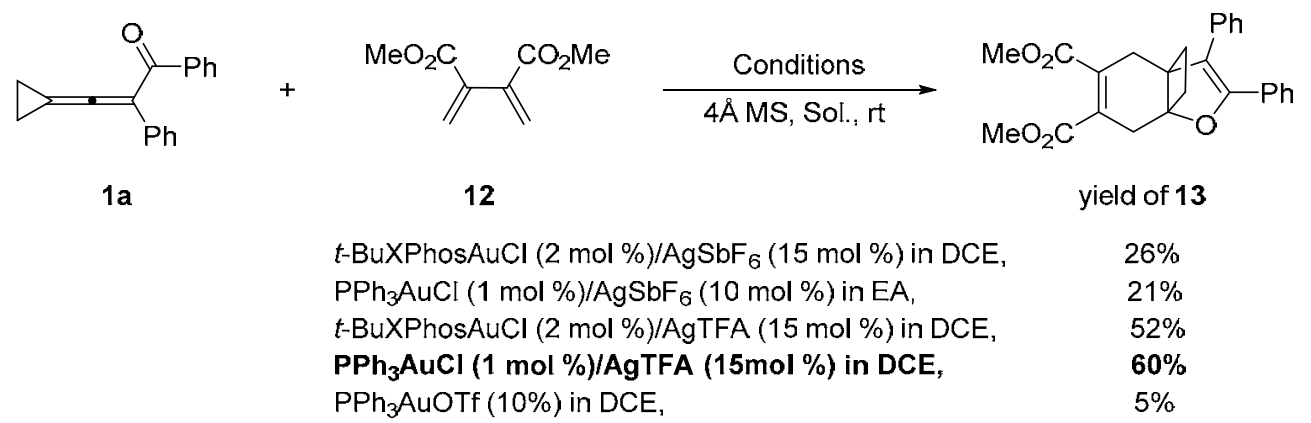

dimethyl 2,3-diphenyl-4,7-dihydro-3a,7a-ethanobenzofuran-5,6-dicarboxylate (13)

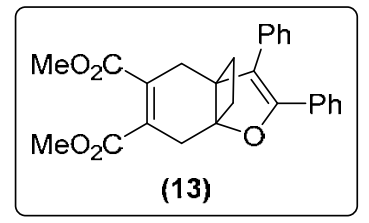

$\mathrm{PPh}_{3} \mathrm{AuCl}$ (1.8 mg, $\left.3.6 \mu \mathrm{mol}, 2 \mathrm{~mol} \%\right)$ and $\mathrm{CF}_{3} \mathrm{CO}_{2} \mathrm{Ag}$ (7.1 mg, $32.3 \mu \mathrm{mol}$, 15 mol\%) were dissolved in $2.0 \mathrm{~mL}$ of dry DCE in a dried vial under $\mathrm{N}_{2}$ atmosphere. Then, $4 \AA$ MS (100 mg) and dimethyl 2,3-dimethylenesuccinate

12 (101.9 mg, $0.5994 \mathrm{mmol}, 3.0$ equiv) were added in this order and the reaction was stirred at room temperature. After being stirred for $5 \mathrm{~min}$, the 3-cyclopropylidene-1,2-diphenylprop-2-en-1-one 1a (49.5 mg, $0.201 \mathrm{mmol}, 1.0$ equiv) was added. The reaction was stirred at room temperature for 20 hours and determined by TLC. After completion, the solution was removed under reduced pressure, and purified by flash silica gel chromatography to afford product $\mathbf{1 3}$ as a colorless oil (50.2 mg, $60 \%) ; \mathrm{R}_{f}=0.16($ Petroleum ether/EtOAc $=20 / 1) ;{ }^{1} \mathrm{H}$ NMR $\left(400 \mathrm{MHz}, \mathrm{CDCl}_{3}\right): \delta$ 7.42-7.38 (m, 2H), 7.30-7.18 (m, 8H), 3.82 (s, 3H), 3.75 (s, 3H), 3.04-2.96 (m, 1H), 2.75-2.45 (m, 4H), 2.44-2.36 (m, 1H), 2.23-2.13 (m, 1H), 1.97-1.85 (m, 1H); $\left.{ }^{13} \mathrm{C} \mathrm{NMR} \mathrm{(100} \mathrm{MHz,} \mathrm{CDCl}_{3}\right): \delta$ 1168.6, 167.6, 151.7, 
137.7, 134.5, 132.5, 131.4, 129.2, 128.5, 128.04, 127.95, 127.6, 126.8, 116.6, 85.0, 55.1, 52.4, 52.2, 34.1, 33.8, 32.4, 30.0; HRMS (ESI) m/z: [M + H] ${ }^{+}$Calcd for $\mathrm{C}_{26} \mathrm{H}_{25} \mathrm{O}_{5}$ 417.1697; Found 417.1695.

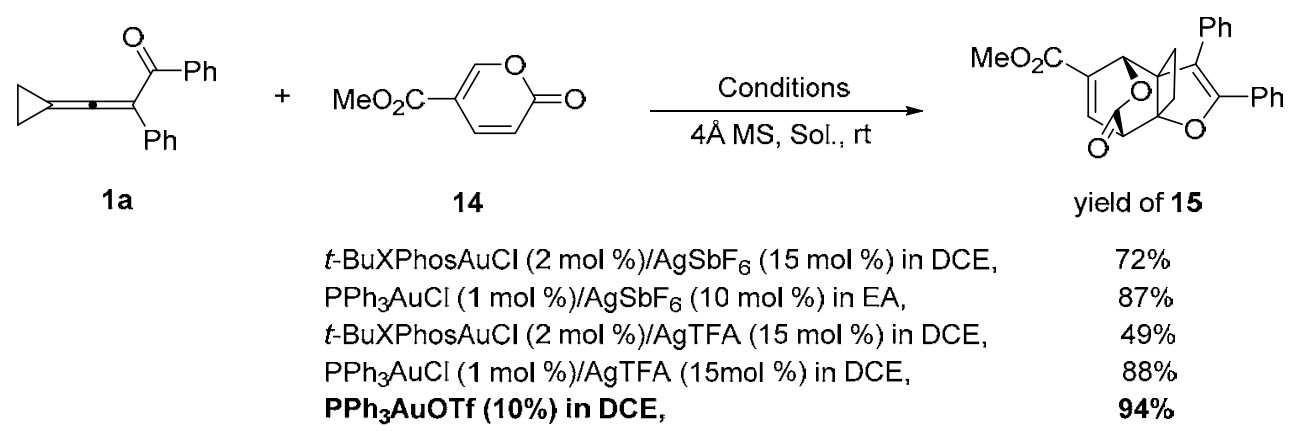

\section{methyl}

(4R,7S)-10-oxo-2,3-diphenyl-4,7-dihydro-4,7-(epoxymethano)-3a,7a-ethanobenzofuran-6-carboxy late (15)

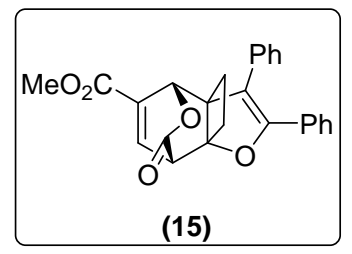

$\mathrm{PPh}_{3} \mathrm{AuOTf}$ (6.4 mg, $10.5 \mu \mathrm{mol}, 10 \mathrm{~mol} \%$ ) was dissolved in $2.0 \mathrm{~mL}$ of dry DCE in a dried vial under $\mathrm{N}_{2}$ atmosphere. Then, $4 \AA$ MS (100 mg) and methyl 2-oxo-2H-pyran-5-carboxylate 14 (15.4 mg, $0.100 \mathrm{mmol}, 1.0$ equiv) were added in this order and the reaction was stirred at room temperature. After being stirred for $5 \mathrm{~min}$, the 3-cyclopropylidene-1,2-diphenylprop-2-en-1-one 1a (27.0 mg, 0.110 mmol, 1.1 equiv) was added. The reaction was stirred at room temperature for 10 hours and determined by TLC. After completion, the solution was removed under reduced pressure, and purified by flash silica gel chromatography to afford product 15 as a white solid (37.7 mg, 94\%), $d r>99: 1$ (ratio was determined by ${ }^{1} \mathrm{H}$ NMR analysis of the crude product); M.p. $130-133{ }^{\circ} \mathrm{C}$ (Petroleum ether/EtOAc); $\mathrm{R}_{f}=0.21$ (Petroleum ether/EtOAc $\left.=20 / 1\right) ;{ }^{1} \mathrm{H}$ NMR $\left(400 \mathrm{MHz}, \mathrm{CDCl}_{3}\right): \delta$ 7.30-7.17 (m, 9H), 7.14-7.07 (m, 2H), 5.83 (d, $J=1.6$ Hz, 1H), 3.86 (s, 1H), 3.84 (s, 3H), 2.80-2.70 (m, 3H), 2.30-2.18 (m, 1H); ${ }^{13} \mathrm{C}$ NMR (100 MHz, $\left.\mathrm{CDCl}_{3}\right): \delta 170.2,162.8,155.0,139.0,134.1,132.7$, 130.3, 129.3, 128.9, 128.20, 128.18, 127.5, 127.4, 114.1, 84.8, 73.8, 56.4, 52.3, 47.9, 28.3, 26.6; HRMS (ESI) m/z: [M + H $]^{+}$Calcd for $\mathrm{C}_{25} \mathrm{H}_{21} \mathrm{O}_{5}$ 401.1384; Found 401.1385.

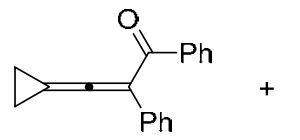

$1 \mathrm{a}$<smiles>CC(=O)c1cc2ccccc2oc1=O</smiles>

16

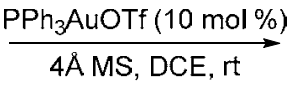<smiles>COC1=C(C)C2(OC1=O)C(=O)Oc1ccccc12</smiles>

17 


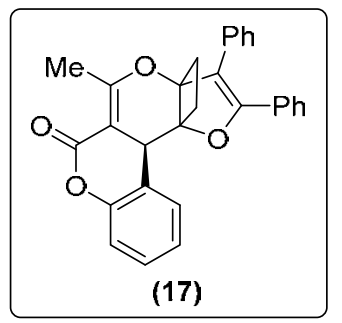

$\mathrm{PPh}_{3} \mathrm{AuOTf}$ (6.4 mg, $10.5 \mu \mathrm{mol}, 10 \mathrm{~mol} \%$ ) was dissolved in $2.0 \mathrm{~mL}$ of dry DCE in a dried vial under $\mathrm{N}_{2}$ atmosphere. Then, $4 \AA \mathrm{MS}(100 \mathrm{mg}$ ) and 3-acetyl-2H-chromen-2-one 16 (20.1 mg, $0.107 \mathrm{mmol}$, 1.0 equiv) were added in this order and the reaction was stirred at room temperature. After being stirred for $5 \mathrm{~min}$, the 3-cyclopropylidene-1,2-diphenylprop-2-en-1-one 1a (24.5 mg, 0.100 mmol, 1.0 equiv) was added. The reaction was stirred at room temperature for 9.5 hours and determined by TLC. After completion, the solution was removed under reduced pressure, and purified by flash silica gel chromatography to afford product $\mathbf{1 7}$ as a white solid (34.9 mg, 80\%), $d r>99: 1$ (ratio was determined by ${ }^{1} \mathrm{H}$ NMR analysis of the crude product); M.p. 187-188 ${ }^{\circ} \mathrm{C}$ (Petroleum ether/EtOAc); $\mathrm{R}_{f}=0.27$ (Petroleum ether/EtOAc $\left.=20 / 1\right) ;{ }^{1} \mathrm{H}$ NMR $\left(400 \mathrm{MHz}, \mathrm{CDCl}_{3}\right): \delta$ $7.50(\mathrm{~d}, J=7.6 \mathrm{~Hz}, 1 \mathrm{H}), 7.37-7.20$ (m, 9H), 7.19-7.10 (m, 3H), 7.08-7.03 (m, 1H), 4.38 (s, 1H), 3.01-2.92 (m, 1H), 2.72-2.58 (m, 2H), 2.55 (d, $J=2.0 \mathrm{~Hz}, 3 \mathrm{H}), 2.53-2.45(\mathrm{~m}, 1 \mathrm{H}) ;{ }^{13} \mathrm{C}$ NMR $(100$ $\left.\mathrm{MHz}, \mathrm{CDCl}_{3}\right): \delta 173.7,161.5,156.0,151.1,132.7,130.0,129.5,129.0,128.6,128.5,128.1,128.0$, 127.3, 126.7, 123.7, 118.7, 117.2, 113.9, 97.6, 88.7, 87.2, 36.7, 29.5, 28.6, 22.5; HRMS (ESI) m/z: $[\mathrm{M}+\mathrm{H}]^{+}$Calcd for $\mathrm{C}_{29} \mathrm{H}_{23} \mathrm{O}_{4}$ 435.1591; Found 435.1591.

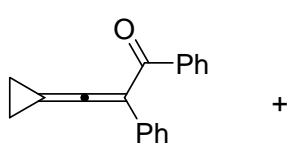

$1 \mathbf{a}$<smiles></smiles>

18 $\underset{4 \AA \mathrm{MS}, \mathrm{DCE}, \mathrm{rt}}{\stackrel{\mathrm{PPh}_{3} \mathrm{AuOTf}(10 \mathrm{~mol} \%)}{\longrightarrow}}$<smiles>[Y9]N1c2c(oc3ccccc23)C(c2ccccc2)C2OC(c3ccccc3)=C(c3ccccc3)C21</smiles>

19

(10S)-2,3,10-triphenyl-4-tosyl-4,10-dihydro-3a,10a-ethanobenzofuro[3,2-b]furo[2,3-e]pyridine

(19)

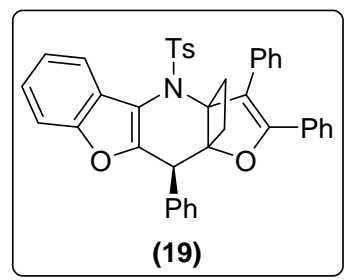

$\mathrm{PPh}_{3} \mathrm{AuOTf}$ (6.5 mg, $10.7 \mu \mathrm{mol}, 10 \mathrm{~mol} \%$ ) was dissolved in $2.0 \mathrm{~mL}$ of dry DCE in a dried vial under $\mathrm{N}_{2}$ atmosphere. Then, $4 \AA \mathrm{MS}(100 \mathrm{mg})$ and N-((Z)-2-((Z)-benzylidene)benzofuran-3(2H)-ylidene)-4-methylbenzenesulfon amide 18 (38.1 mg, $0.102 \mathrm{mmol}, 1.0$ equiv) were added in this order and the reaction was stirred at room temperature. After being stirred for 5 min, the 3-cyclopropylidene-1,2-diphenylprop-2-en-1-one 1a (24.3 mg, $0.100 \mathrm{mmol}, 1.0$ equiv) was added. The reaction was stirred at room temperature for 1 hour and determined by TLC. After completion, the solution was removed under reduced pressure, and purified by flash silica gel chromatography to 
afford product 19 as a light yellow solid (57.6 mg, 92\%), $d r>99: 1$ (ratio was determined by ${ }^{1} \mathrm{H}$ NMR analysis of the crude product); M.p. 232-233 ${ }^{\circ} \mathrm{C}$ (Petroleum ether/DCM); $\mathrm{R}_{f}=0.42$ (Petroleum ether/DCM = 1/1); ${ }^{1} \mathrm{H}$ NMR (400 MHz, $\left.\mathrm{CDCl}_{3}\right): \delta$ 8.05-7.98 (m, 1H), 7.52-7.36 (m, 13H), 7.34-7.23 (m, 2H), 7.20-7.11 (m, 5H), 7.09-6.91 (m, 2H), 3.81 (s, 1H), 3.01-2.90 (m, 1H), 2.85-2.76 (m, 1H), 2.40 (s, 3H), 2.32-2.13 (m, 2H); ${ }^{13} \mathrm{C}$ NMR (100 MHz, $\left.\mathrm{CDCl}_{3}\right): \delta 153.8,152.1,151.5,143.4,137.2$, 133.0, 131.2, 131.0, 130.8, 130.2, 129.5, 128.7, 128.6, 128.3, 128.1, 127.83, 127.79, 127.2, 126.9, 124.8, 124.0, 123.5, 121.2, 118.3, 116.8, 111.7, 93.7, 78.1, 46.0, 26.4, 26.0, 21.5; HRMS (ESI) m/z: $[\mathrm{M}+\mathrm{H}]^{+}$Calcd for $\mathrm{C}_{40} \mathrm{H}_{32} \mathrm{NO}_{4} \mathrm{~S}$ 622.2047; Found 622.2054.
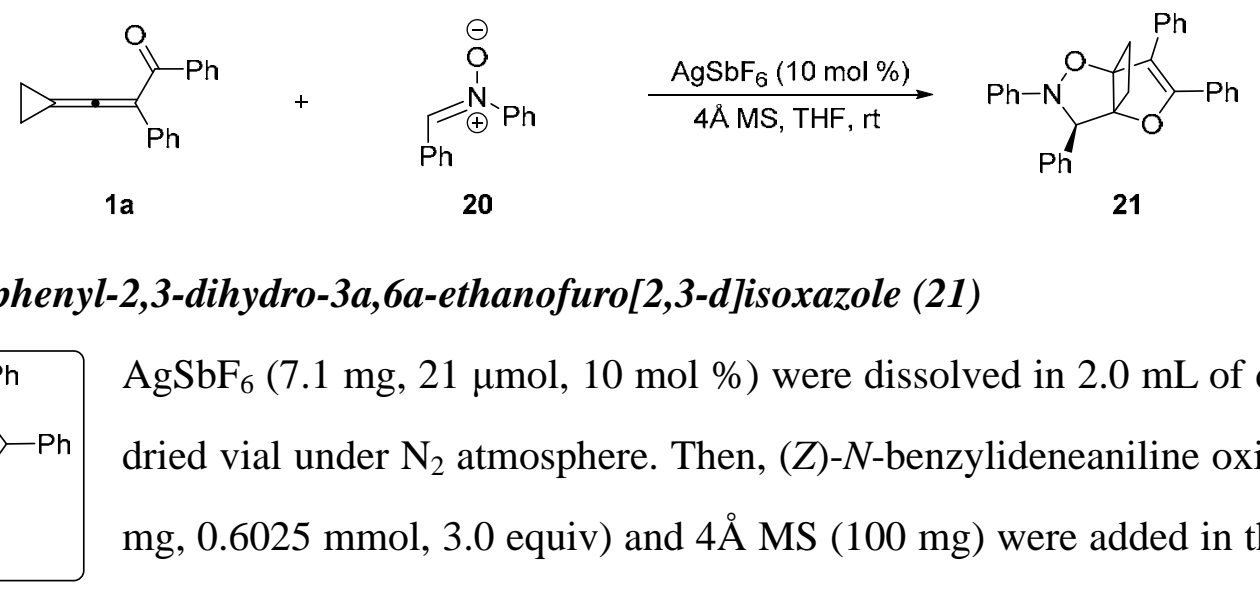

\section{2,3,5,6-tetraphenyl-2,3-dihydro-3a,6a-ethanofuro[2,3-d]isoxazole (21)}

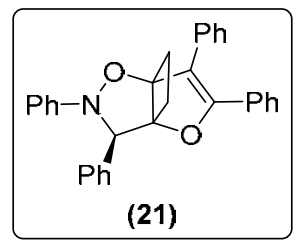

$\operatorname{AgSbF}_{6}(7.1 \mathrm{mg}, 21 \mu \mathrm{mol}, 10 \mathrm{~mol} \%)$ were dissolved in $2.0 \mathrm{~mL}$ of dry THF in a dried vial under $\mathrm{N}_{2}$ atmosphere. Then, $(Z)-N$-benzylideneaniline oxide 20 (118.7 mg, $0.6025 \mathrm{mmol}, 3.0$ equiv) and $4 \AA$ MS (100 mg) were added in this order and the reaction was stirred at room temperature. After being stirred for $5 \mathrm{~min}$, the 3-cyclopropylidene-1,2-diphenylprop-2-en-1-one 1a (49.8 mg, $0.202 \mathrm{mmol}, 1.0$ equiv) was added. The reaction was stirred at room temperature for 48 hours and determined by TLC. After completion, the solution was removed under reduced pressure, and purified by flash silica gel chromatography to afford 21 as a yellow solid (42.8 mg, 48\%); $d r>20: 1$ (ratio was determined by ${ }^{1} \mathrm{H}$ NMR analysis of the crude product); M.p. $138-139{ }^{\circ} \mathrm{C}\left(\right.$ Petroleum ether/EtOAc); $\mathrm{R}_{f}=0.73$ (Petroleum ether $/$ EtOAc $=$ 10/1); ${ }^{1} \mathrm{H}$ NMR (400 MHz, $\mathrm{CDCl}_{3}$ ): $\delta 7.57$ (d, $\left.J=7.6 \mathrm{~Hz}, 2 \mathrm{H}\right), 7.41$ (t, $\left.J=7.4 \mathrm{~Hz}, 4 \mathrm{H}\right), 7.36-7.20$ (m, 8H), 7.19-7.09 (m, 5H), 6.92 (t, $J=7.0 \mathrm{~Hz}, 1 \mathrm{H}), 4.98$ (s, 1H), 2.47-2.29 (m, 2H), 2.00 (t, $J=8.4 \mathrm{~Hz}$, 2H); $\left.{ }^{13} \mathrm{C} \mathrm{NMR} \mathrm{(100} \mathrm{MHz,} \mathrm{CDCl}_{3}\right): \delta 156.4,149.7,136.7,132.7,131.2,129.5,128.7,128.5,128.4$, 128.2, 127.83, 127.75, 127.4, 126.5, 122.7, 117.6, 113.4, 97.9, 93.1, 75.3, 26.8, 22.4; HRMS $\left(\mathrm{ES}^{+}\right.$-TOF) calcd for $\mathrm{C}_{31} \mathrm{H}_{26} \mathrm{NO}_{2}\left([\mathrm{M}+\mathrm{H}]^{+}\right): 444.1958$, found 444.1965.

\section{X-ray diffraction analysis of 4a, 5a, 5h' and 21}

(a) Method for crystal growth of 4a, 5a, 5h' and 21:

$0.040 \mathrm{mmol}$ of $\mathbf{4 a}, \mathbf{5 a}, \mathbf{5 h}$ ' and 21 were dissolved in $1.0 \mathrm{ml}$ of solvent (Petroleum ether/EtOAc/DCM 
$=3 / 1 / 1$ ) in $2.0 \mathrm{ml}$ sample bottle. After about 12 hours of natural volatilization at room temperature, single crystal could be obtained.

\section{(b) Crystallographic structure analysis of 4a, 5a, 5h' and 21:}

A suitable single crystal was mounted on a Xcalibur, Atlas, Gemini ultra at 296(2), 100.01(10), 296(2) and $296 \mathrm{~K}$, using Mo Ka radiation $(\lambda(\mathbf{4 a}, \mathbf{5 h}$ ' and $\mathbf{2 1})=0.71073 \AA$ or $\lambda(\mathbf{5 a})=1.54184 \AA)$. The intensity data were collected with CrysAlisPro program and reduced by CrysAlisPro program. The structure was solved by direct methods, expended by difference Fourier syntheses and refined by Full-matrix squares on $\mathrm{F}^{2}$ using SHELXL program packages. All non-hydrogen atoms were refined anisotropically. Hydrogen atoms were placed in ideal positions and refined as riding atoms. Details of the X-ray experiments and crystal data are summarized in Table S3, Table S4, Table S5 and

\section{Table S6.}

Figure S1. ORTEP drawing of 4a with ellipsoid contour at 30\% probability level (CCDC: 2073826)
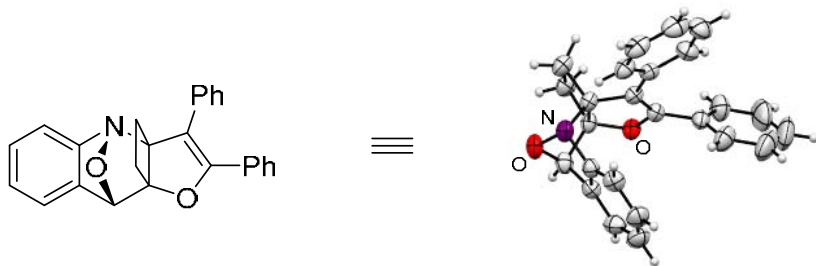

Figure S2. ORTEP drawing of 5a with ellipsoid contour at 30\% probability level (CCDC: 2073827)
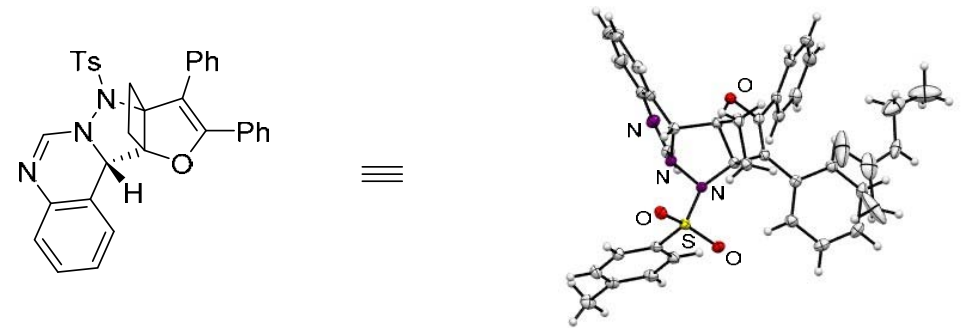
Figure S3. ORTEP drawing of diastereoisomer $\mathbf{5 h}$ ' with ellipsoid contour at 30\% probability level (CCDC: 2073828)
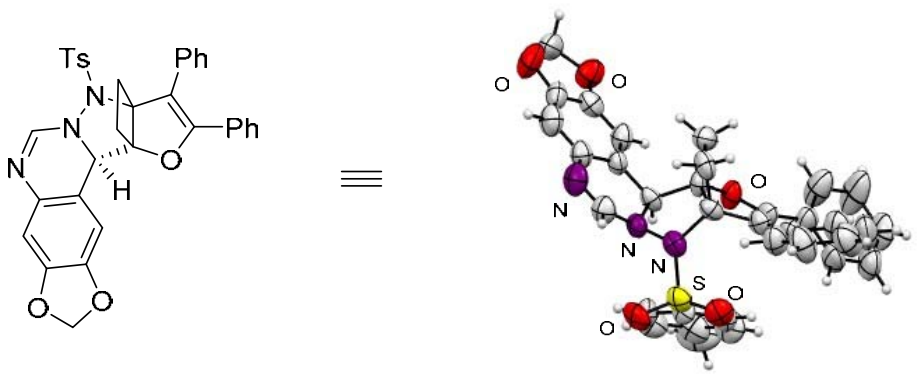

Figure S4. ORTEP drawing of $\mathbf{2 1}$ with ellipsoid contour at 30\% probability level (CCDC: 1962639)<smiles>C#CCC1(c2ccccc2)OC2(C)C(c3ccccc3)=C(c3ccccc3)ON2c2ccccc21</smiles>

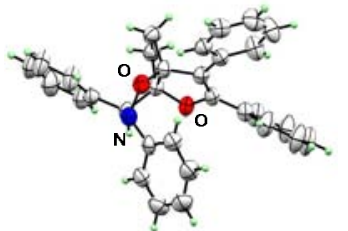


Table S3. Crystal data and structure refinement for $\mathbf{4 a}$.

Empirical formula

Formula weight

Temperature

Wavelength

Crystal system, Space group

Unit cell dimensions

Volume

Z

Density(calculated)

Absorption coefficient

$\mathrm{F}(000)$

Crystal size

Theta range for data collection

Index ranges

Reflections collected

Independent reflections

Completeness to theta $=25.242^{\circ}$

Absorption correction

Max. and min. transmission

Refinement method

Data/restraints/parameters

Goodness-of-fit on $\mathrm{F}^{2}$

Final R indices [I>2sigma(I)]

$\mathrm{R}$ indices(all data)

Extinction coefficient

Largest diff. peak and hole
$\mathrm{C}_{25} \mathrm{H}_{19} \mathrm{NO}_{2}$

365.41

296(2) K

$0.71073 \AA$

monoclinic, $C 2 / c$

$\mathrm{a}=24.545(7) \AA \quad \alpha=90^{\circ}$

$b=16.702(5) \AA \quad \beta=102.670(4)^{\circ}$

$c=9.528(3) \AA \quad \gamma=90^{\circ}$

3810.9(18) $\AA^{3}$

8

$1.274 \mathrm{~g} / \mathrm{cm}^{3}$

$0.081 \mathrm{~mm}^{-1}$

1536

$0.180 * 0.150 * 0.120 \mathrm{~mm}^{3}$

1.486 to $27.562^{\circ}$.

$-25<=\mathrm{h}<=31,-18<=\mathrm{k}<=21,-12<=\mathrm{l}<=12$

11463

$4355[\mathrm{R}(\mathrm{int})=0.0317]$

$99.6 \%$

?

? and ?

Full-matrix squares on $\mathrm{F}^{2}$

$4355 / 1 / 253$

1.041

$\mathrm{R}_{1}=0.0521, \mathrm{wR}_{2}=0.1517$

$\mathrm{R}_{1}=0.0799, \mathrm{wR}_{2}=0.1720$

0.214 and $-0.254 \mathrm{e} \AA^{3}$ 
Table S4. Crystal data and structure refinement for $\mathbf{5 a}$.

Empirical formula

Formula weight

Temperature

Wavelength

Crystal system, Space group

Unit cell dimensions

Volume

Z

Density(calculated)

Absorption coefficient

$\mathrm{F}(000)$

Crystal size

Theta range for data collection

Index ranges

Reflections collected

Independent reflections

Completeness to theta $=67.684^{\circ}$

Absorption correction

Max. and min. transmission

Refinement method

Data/restraints/parameters

Goodness-of-fit on $\mathrm{F}^{2}$

Final R indices [I $>2 \operatorname{sigma}(\mathrm{I})]$

$\mathrm{R}$ indices(all data)

Extinction coefficient

Largest diff. peak and hole
$\mathrm{C}_{33} \mathrm{H}_{27} \mathrm{~N}_{3} \mathrm{O}_{3} \mathrm{~S}$

545.18

100.01(10) K

$1.54184 \AA$

monoclinic, $P 1$ 21/c 1

$\mathrm{a}=18.5186(3) \AA \quad \alpha=90^{\circ}$

$\mathrm{b}=14.4787(2) \AA \quad \beta=98.6580(10)^{\circ}$

$\mathrm{c}=12.1998(2) \AA \quad \gamma=90^{\circ}$

3233.80(9) $\AA^{3}$

4

$1.300 \mathrm{~g} / \mathrm{cm}^{3}$

$1.265 \mathrm{~mm}^{-1}$

1336

$0.200 * 0.200 * 0.200 \mathrm{~mm}^{3}$

2.413 to $75.285^{\circ}$.

$-23<=\mathrm{h}<=20,-17<=\mathrm{k}<=18,-14<=\mathrm{l}<=15$

18348

$6449[\mathrm{R}(\mathrm{int})=0.0334]$

$99.8 \%$

multi-scan

1.00000 and 0.79384

Full-matrix squares on $\mathrm{F}^{2}$

$6449 / 0 / 418$

1.082

$\mathrm{R}_{1}=0.0514, \mathrm{wR}_{2}=0.1383$

$\mathrm{R}_{1}=0.0568, \mathrm{wR}_{2}=0.1450$

0.1.332 and -0.726e $\AA^{3}$ 
Table S5. Crystal data and structure refinement for diastereoisomer $\mathbf{5 h}$ '.

Empirical formula

Formula weight

Temperature

Wavelength

Crystal system, Space group

Unit cell dimensions

Volume

Z

Density(calculated)

Absorption coefficient

$\mathrm{F}(000)$

Crystal size

Theta range for data collection

Index ranges

Reflections collected

Independent reflections

Completeness to theta $=24.000^{\circ}$

Absorption correction

Max. and min. transmission

Refinement method

Data/restraints/parameters

Goodness-of-fit on $\mathrm{F}^{2}$

Final R indices [I $>2 \operatorname{sigma}(\mathrm{I})]$

$\mathrm{R}$ indices(all data)

Extinction coefficient

Largest diff. peak and hole
$\mathrm{C}_{34} \mathrm{H}_{27} \mathrm{~N}_{3} \mathrm{O}_{5} \mathrm{~S}$

589.17

296(2) K

$0.71073 \AA$

triclinic, $P-1$

$\mathrm{a}=10.303(17) \AA \quad \alpha=90.926(15)^{\circ}$

$\mathrm{b}=10.575(17) \AA \quad \beta=107.172(15)^{\circ}$

$c=16.85(3) \AA \quad \gamma=106.236(15)^{\circ}$

1675(5) $\AA^{3}$

2

$1.406 \mathrm{~g} / \mathrm{cm}^{3}$

$0.383 \mathrm{~mm}^{-1}$

732

$0.100 * 0.090 * 0.80 \mathrm{~mm}^{3}$

2.167 to $24.000^{\circ}$.

$-11<=\mathrm{h}<=11,-12<=\mathrm{k}<=12,-19<=\mathrm{l}<=19$

11348

5225 [R(int) $=0.1597]$

$99.3 \%$

?

? and ?

Full-matrix squares on $\mathrm{F}^{2}$

$5225 / 0 / 425$

1.009

$\mathrm{R}_{1}=0.1142, \mathrm{wR}_{2}=0.2365$

$\mathrm{R}_{1}=0.2814, \mathrm{wR}_{2}=0.3112$

0.387 and $-0.495 e \AA^{3}$ 
Table S6. Crystal data and structure refinement for 21.

Empirical formula

Formula weight

Temperature

Wavelength

Crystal system, Space group

Unit cell dimensions

Volume

$\mathrm{Z}$

Density(calculated)

Absorption coefficient

$\mathrm{F}(000)$

Crystal size

Theta range for data collection

Index ranges

Reflections collected

Independent reflections

Completeness to theta $=25.346^{\circ}$

Absorption correction

Max. and min. transmission

Refinement method

Data/restraints/parameters

Goodness-of-fit on $\mathrm{F}^{2}$

Final R indices [I > 2sigma(I)]

$\mathrm{R}$ indices(all data)

Extinction coefficient

Largest diff. peak and hole
$\mathrm{C}_{31} \mathrm{H}_{25} \mathrm{NO}_{2}$

443.52

\section{$296 \mathrm{~K}$}

$0.71073 \AA$

monoclinic, $C_{1} 2 / c 1$

$\mathrm{a}=26.0521(18) \AA \quad \alpha=90^{\circ}$

$b=13.0936(8) \AA \quad \beta=108.123(8)^{\circ}$

$\mathrm{c}=14.6011(11) \AA \quad \gamma=90^{\circ}$

4733.6(6) $\AA^{3}$

8

$1.245 \mathrm{~g} / \mathrm{m}^{3}$

$0.077 \mathrm{~mm}^{-1}$

1872.0

$0.42 * 0.40 * 0.26 \mathrm{~mm}^{3}$

2.121 to $25.346^{\circ}$.

$-30<=\mathrm{h}<=31,-12<=\mathrm{k}<=15,-11<=\mathrm{l}<=17$

9024

$4332[\mathrm{R}(\mathrm{int})=0.0310]$

$99.8 \%$

Multi-scan from equivalents

1.0000 and 0.93236

Full-matrix squares on $\mathrm{F}^{2}$

$4332 / 0 / 307$

1.024

$\mathrm{R}_{1}=0.0897, \mathrm{wR}_{2}=0.1206$

$\mathrm{R}_{1}=0.0516, \mathrm{wR}_{2}=0.1449$

0.154 and $-0.192 \mathrm{e} \AA^{3}$ 


\section{Reference}

1. Zhang, J.; Qian, D.; Hu, H.; Liu, F.; Tang, B.; Ye, W.; Wang, Y. Angew. Chem. Int. Ed. 2014, 53, 13751-13755.

2. Taichi Tamai,; Keiko Fujiwara,; Shinya Higashimae,; Akihiro Nomoto,; and Akiya Ogawa. Org. Lett. 2016, 18, 2114-2117.

3. Zalesskiy, S. S.; Sedykh, A. E.; Kashin, A. S.; Ananikov, V. P. J. Am. Chem. Soc. 2013, 135, 3550-3559.

4. Rene E. Ebule,; Deepika Malhotra,; Gerald B. Hammond,; Bo Xu. Adv. Synth. Catal. 2016, 358, 1478-1481.

5. Collado, A.; Gómez-Suárez, A.; Martin, A. R.; Slawin, A. M. Z.; Nolan, S. P. Chem. Commun. 2013, 49, 5541-5543.

6. Miao, M.; Cao, J.; Zhang, J.; Huang, X.; Wu, L. Org. Lett. 2012, 14, 2718.

7. Wang, F.; Xu, P.; Wang, S.; Ji, S.; Org. Lett. 2018, 20, 2204-2207.

8. Zhou, L.; Yuan, C.; Zeng, Y.; Wang, Q.; Wang, C.; Liu, M. Wang, W.; Wu, Y.; Zheng, B.; Guo, H. Org. Lett. 2019, 21, 4882-4886.

9. Hong, Y.; Chen, J.; Zhang, Z.; Liu, Y.; Zhang, W. Org. Lett. 2016, 18, 2640-2643.

10. Gu, Z.; Zhou, J.; Jiang, G.; Zhou, Y. Org. Chem. Front. 2018, 5(7), 1148-1151.

11. Liu, F.; Yu, Y.; Zhang, J. Angew. Chem. Int. Ed. 2009, 48, 5505-5508.

9. Copies of ${ }^{1} \mathrm{H} N M R$ and ${ }^{13} \mathrm{C} N M R$ 
${ }^{1} \mathrm{H}$ NMR $\left(400 \mathrm{MHz}, \mathrm{CDCl}_{3}\right)$

\subsection{1}

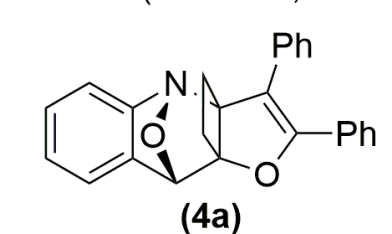

(4a)

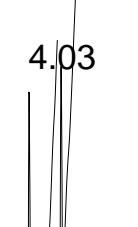


${ }^{13} \mathrm{C}$ NMR $\left(100 \mathrm{MHz}, \mathrm{CDCl}_{3}\right)$

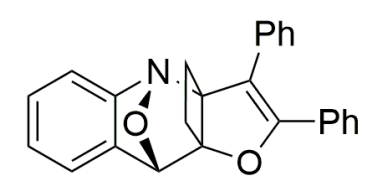

(4a) 
5.00
${ }^{1} \mathrm{H}$ NMR $\left(400 \mathrm{MHz}, \mathrm{CDCl}_{3}\right)$

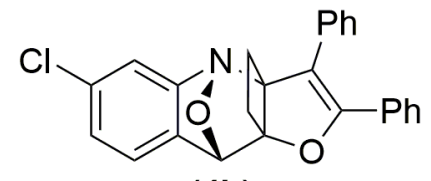

(4b)
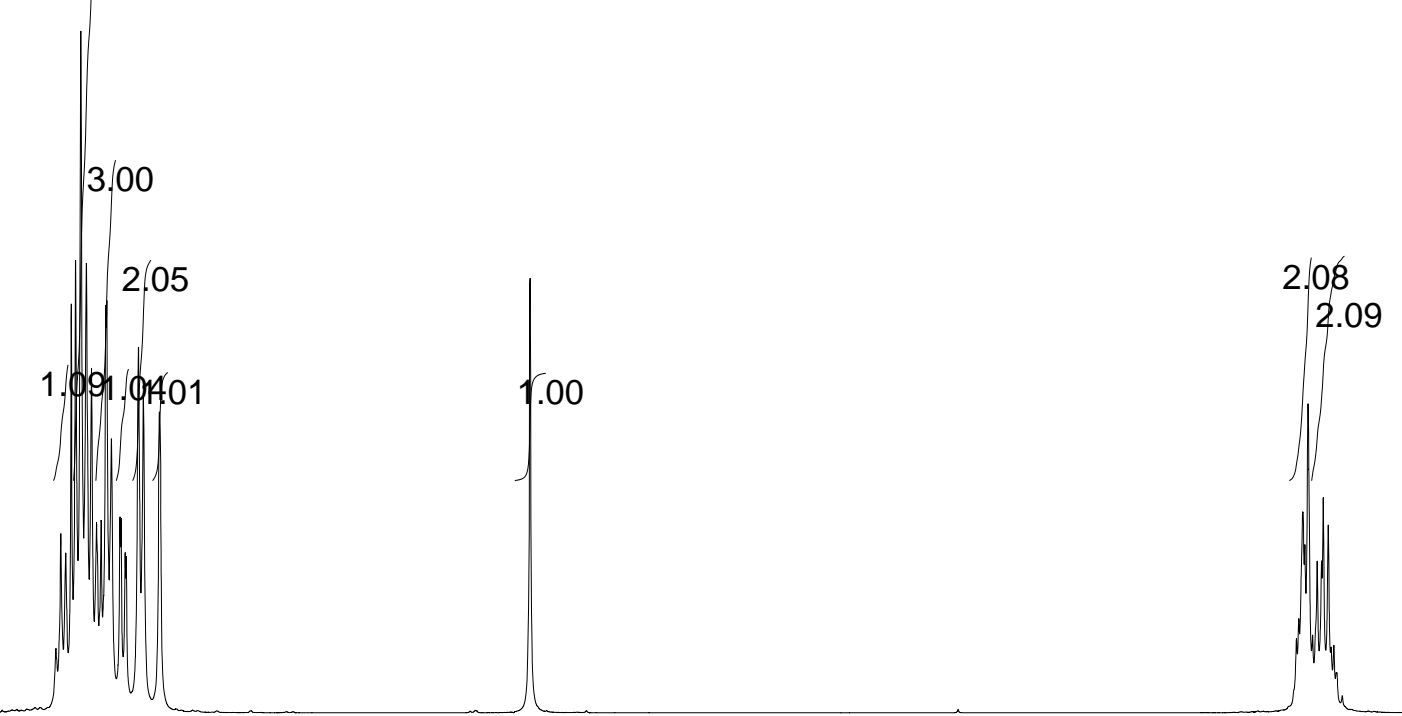

2.09 
${ }^{13} \mathrm{C}$ NMR $\left(100 \mathrm{MHz}, \mathrm{CDCl}_{3}\right)$

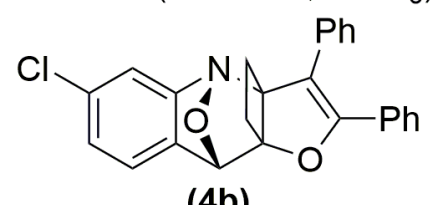

(4b)

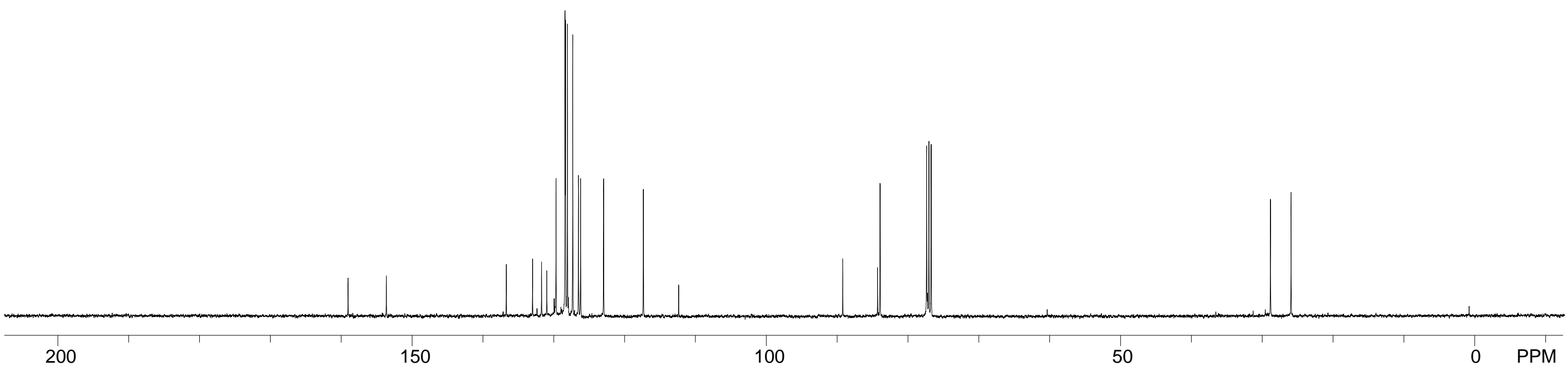


7.01

2.04

2.03

1.061 .00
${ }^{1} \mathrm{H}$ NMR $\left(400 \mathrm{MHz}, \mathrm{CDCl}_{3}\right)$

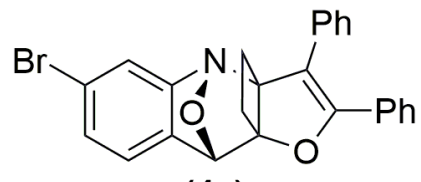

(4c) 

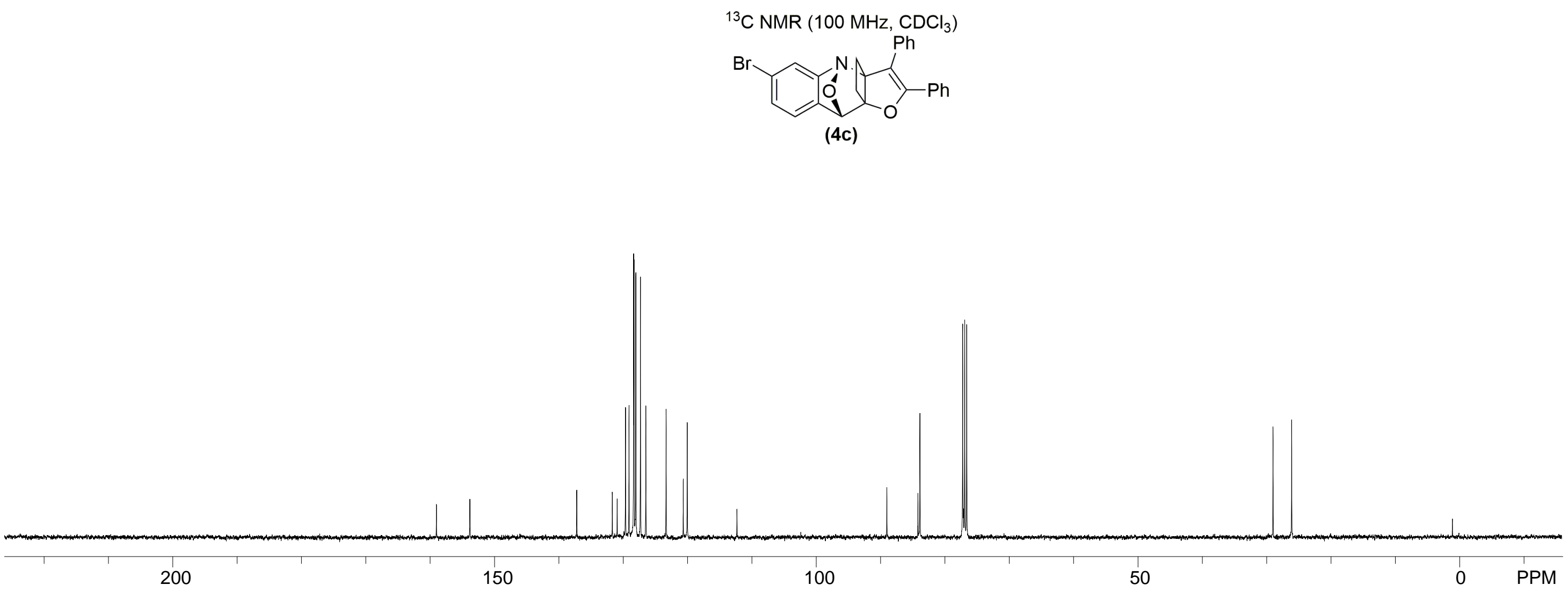
${ }^{1} \mathrm{H}$ NMR $\left(400 \mathrm{MHz}, \mathrm{CDCl}_{3}\right)$

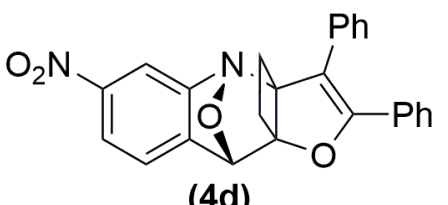

(4d)

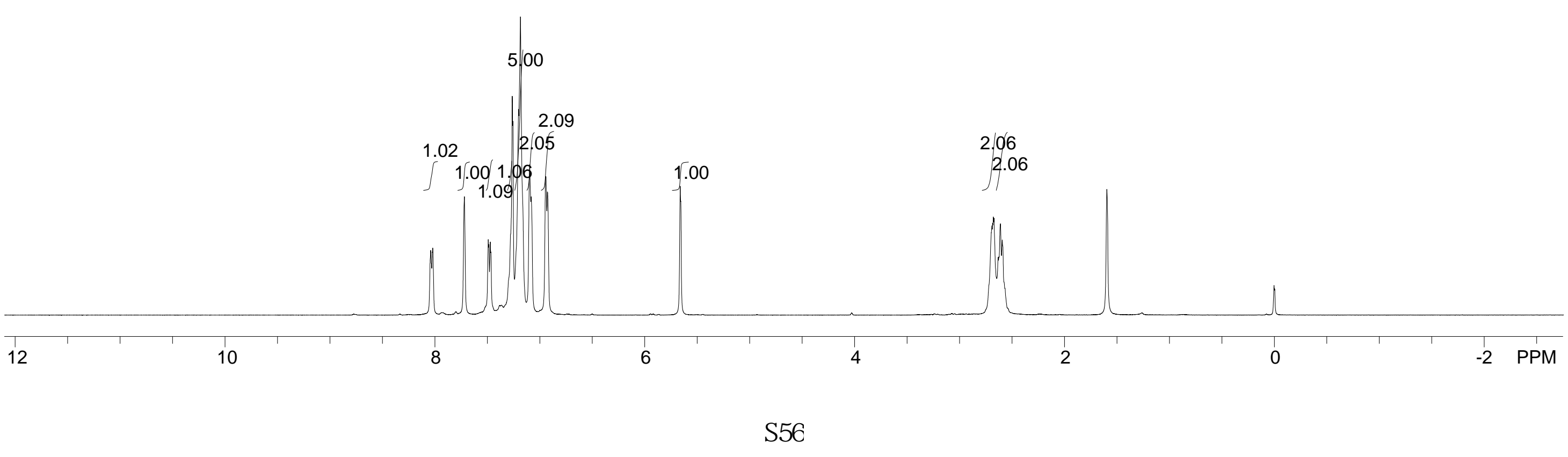


${ }^{13} \mathrm{C} \mathrm{NMR}\left(100 \mathrm{MHz}, \mathrm{CDCl}_{3}\right)$

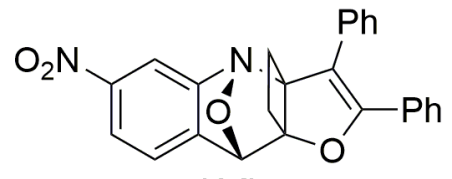

(4d) 
${ }^{1} \mathrm{H} \mathrm{NMR}\left(400 \mathrm{MHz}, \mathrm{CDCl}_{3}\right)$

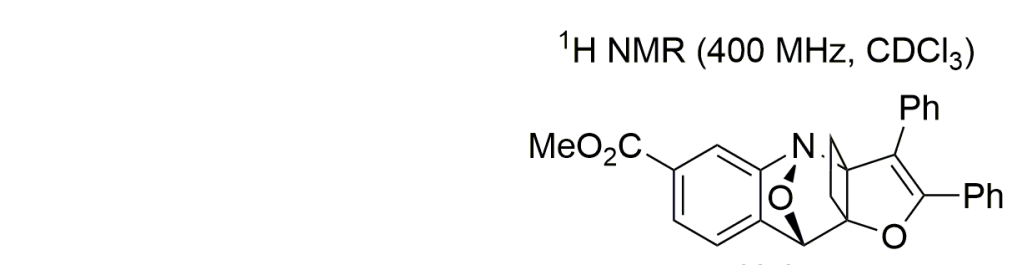

(4e)

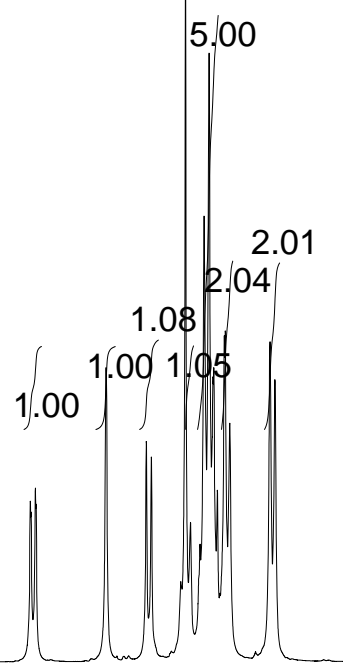




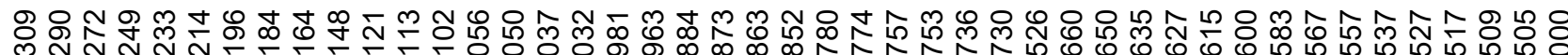

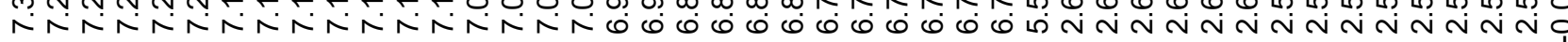
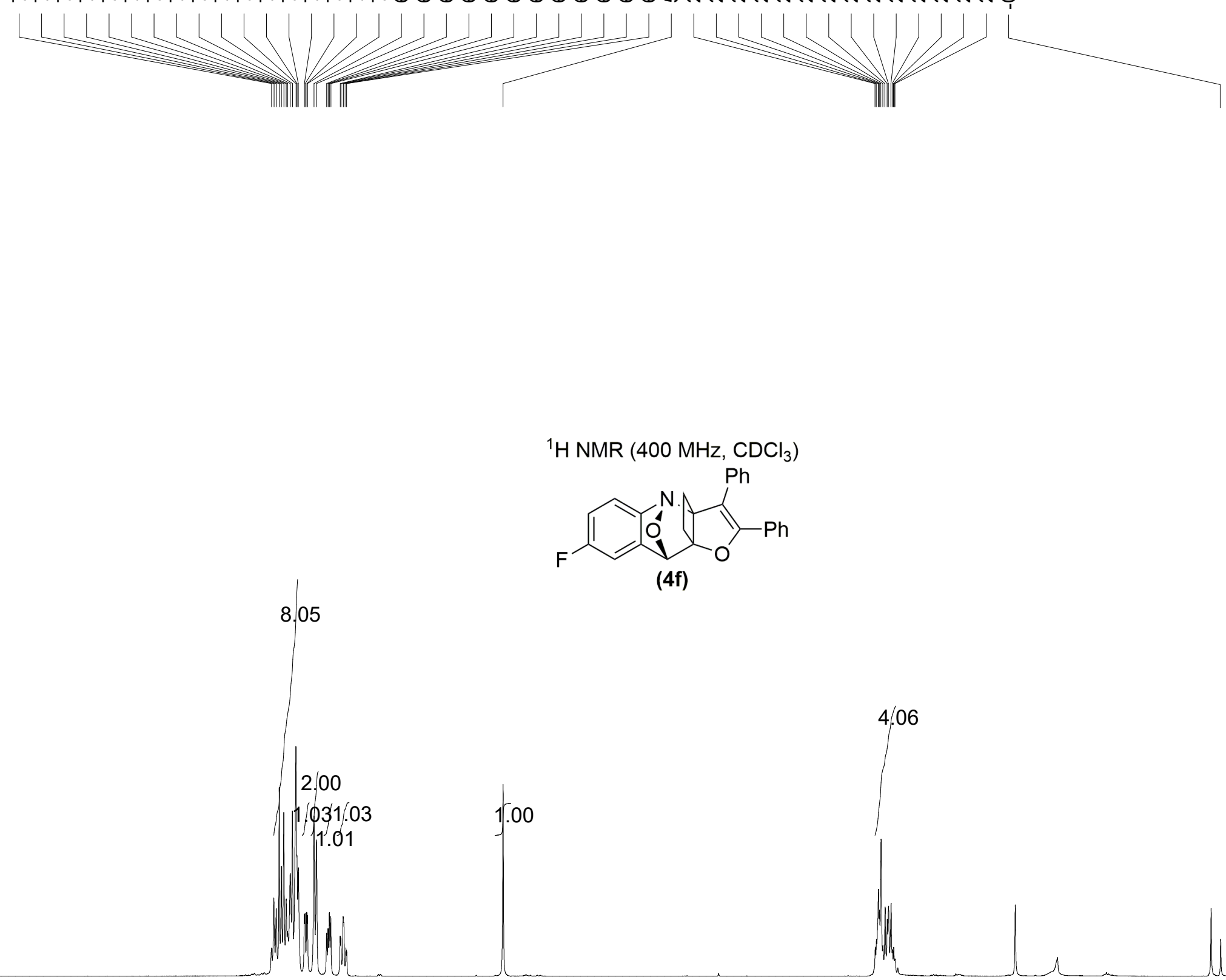

${ }^{1} \mathrm{H} \mathrm{NMR}\left(400 \mathrm{MHz}, \mathrm{CDCl}_{3}\right)$

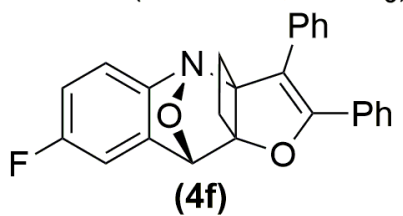


${ }^{13} \mathrm{C}$ NMR $\left(100 \mathrm{MHz}, \mathrm{CDCl}_{3}\right)$

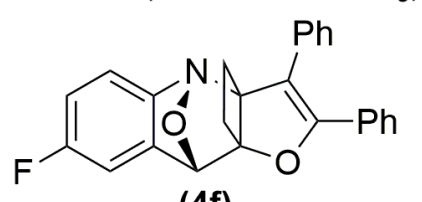

(4f) 


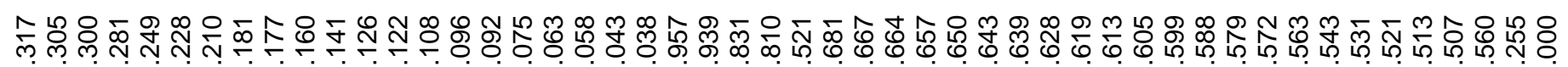

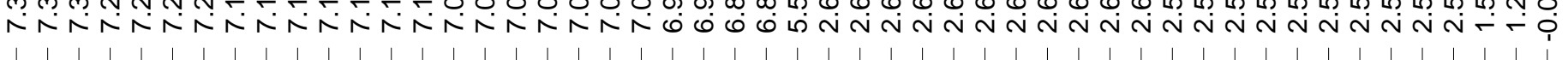

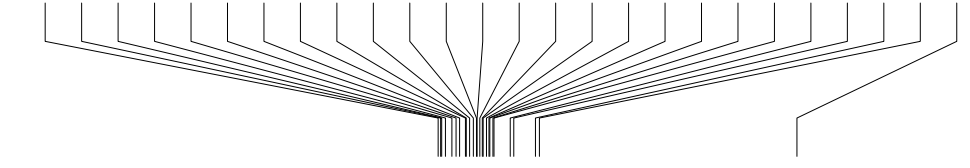

${ }^{1} \mathrm{H} \mathrm{NMR}\left(400 \mathrm{MHz}, \mathrm{CDCl}_{3}\right)$
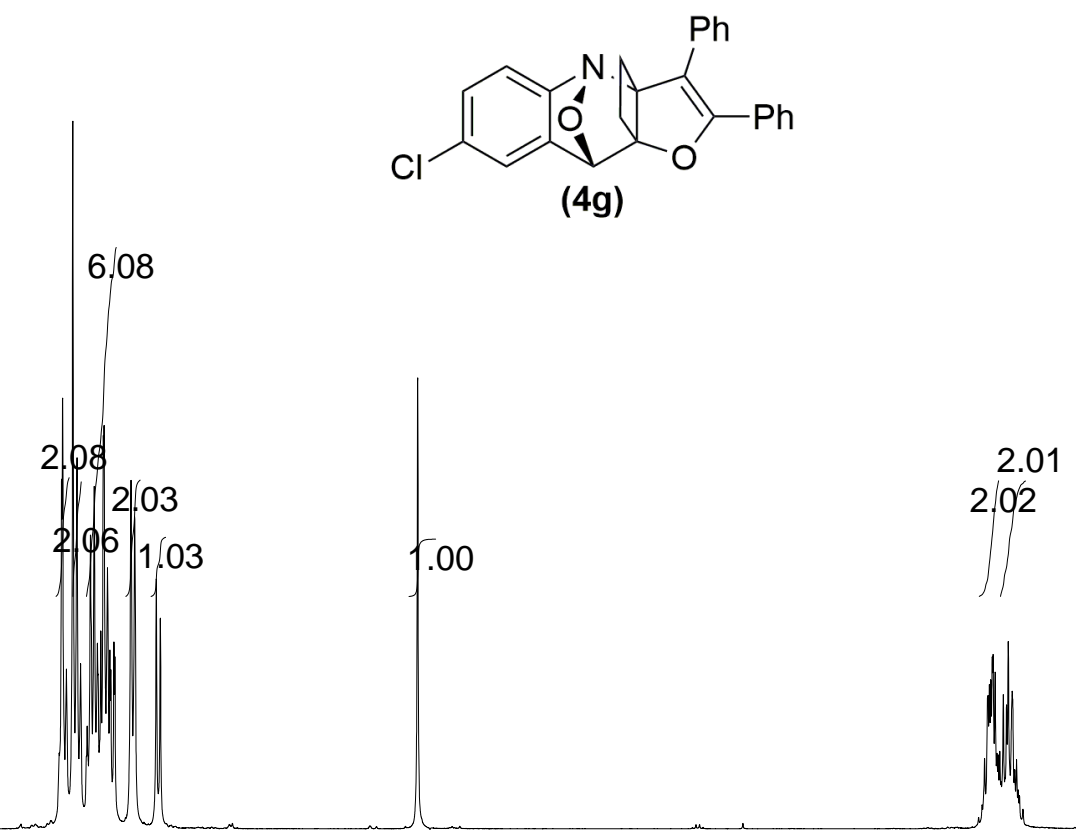
${ }^{13} \mathrm{C}$ NMR $\left(100 \mathrm{MHz}, \mathrm{CDCl}_{3}\right)$

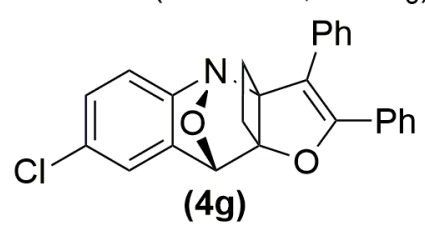


5.02

3.00

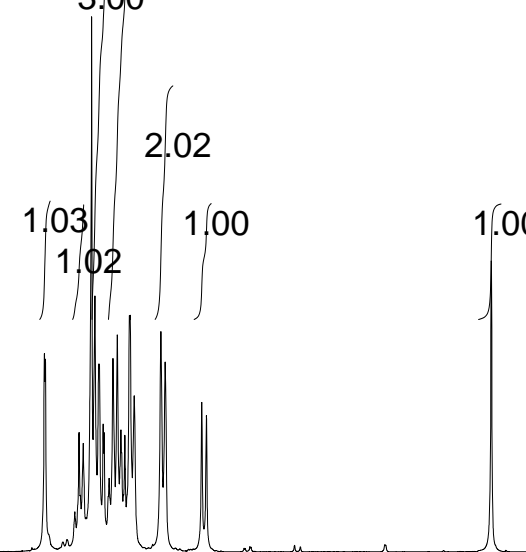

${ }^{1} \mathrm{H}$ NMR $\left(400 \mathrm{MHz}, \mathrm{CDCl}_{3}\right)$

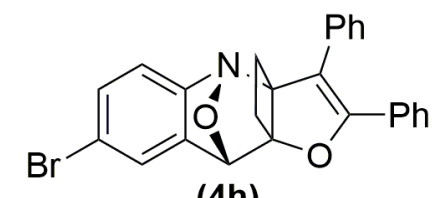

(4h) 
${ }^{13} \mathrm{C} \mathrm{NMR}\left(100 \mathrm{MHz}, \mathrm{CDCl}_{3}\right)$

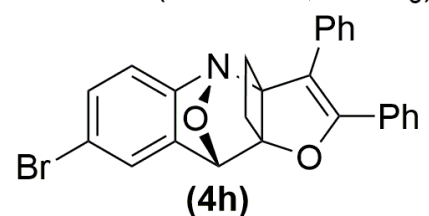

(4h) 


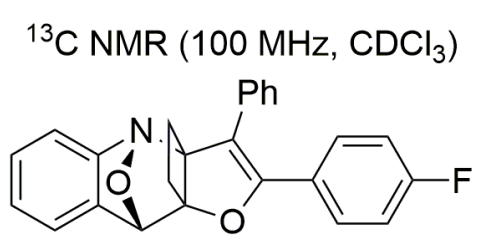

(4i)

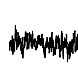




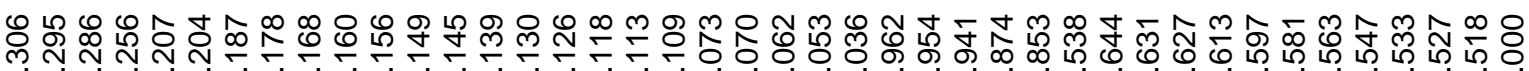

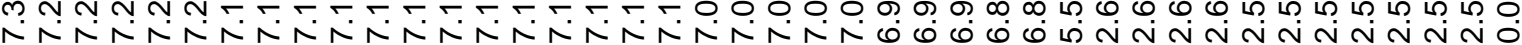

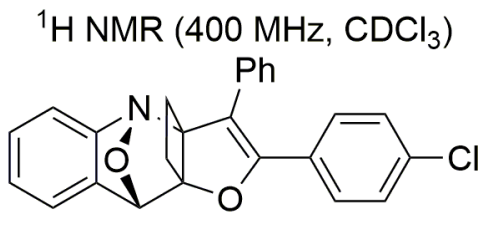

(4j)

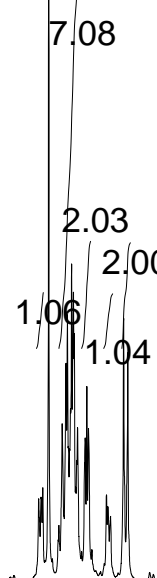

2.04

2.06 


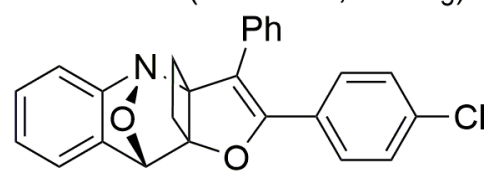

(4j) 

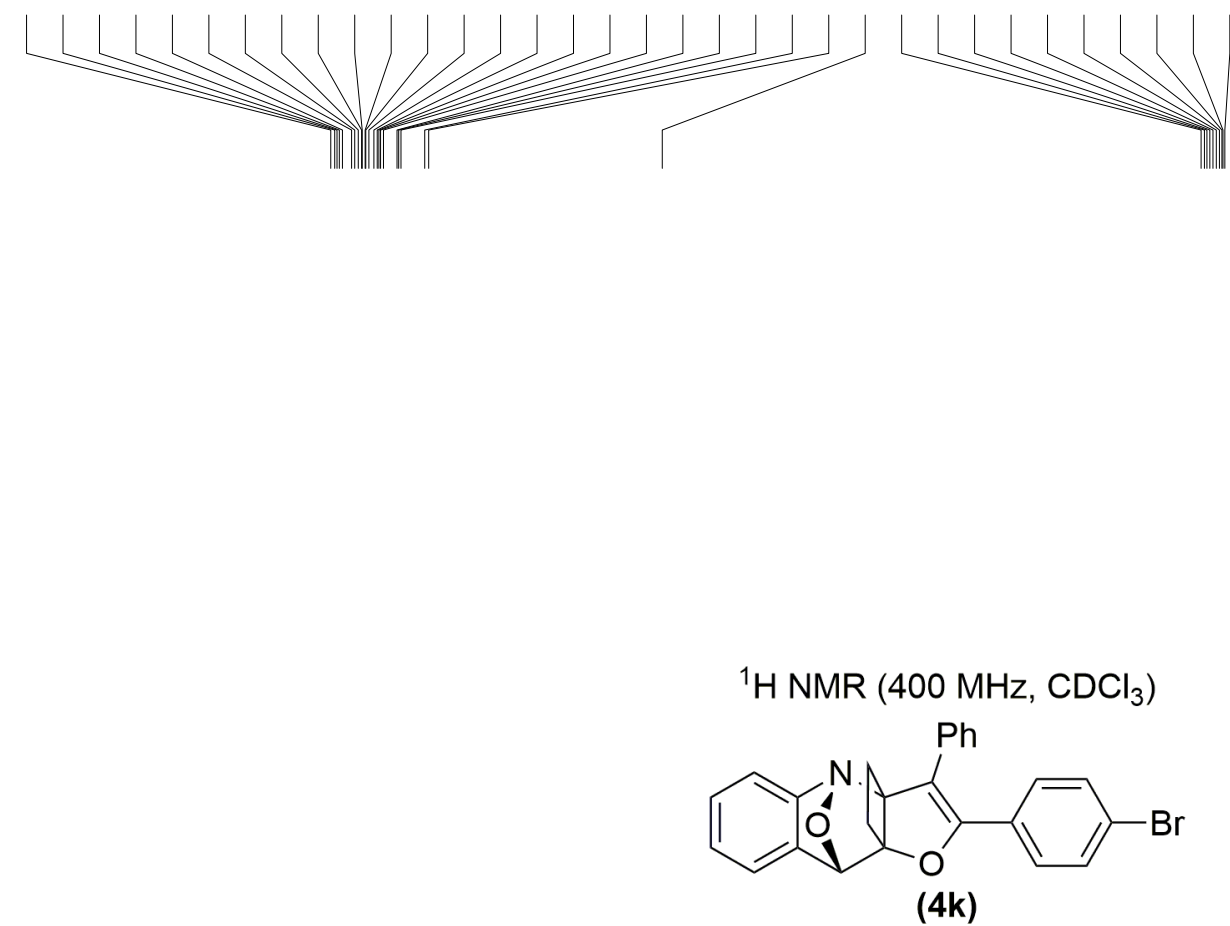

.09

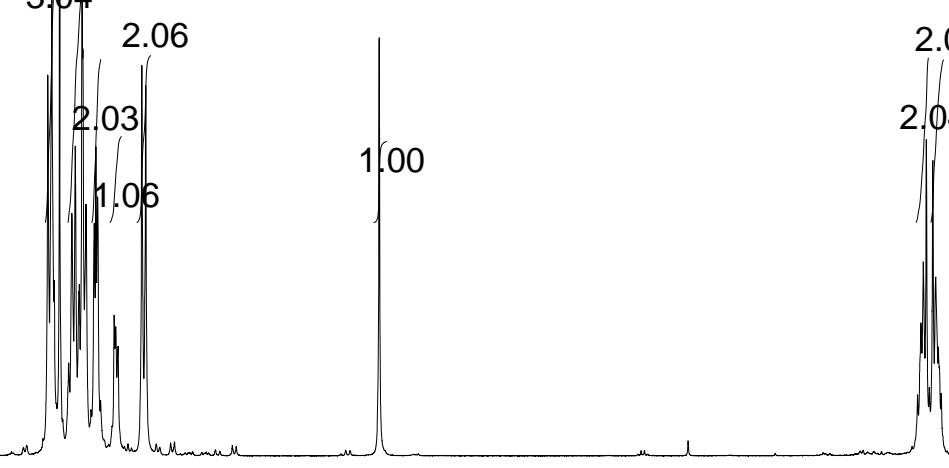

2.04 
${ }^{13} \mathrm{C} \mathrm{NMR}\left(100 \mathrm{MHz}, \mathrm{CDCl}_{3}\right)$

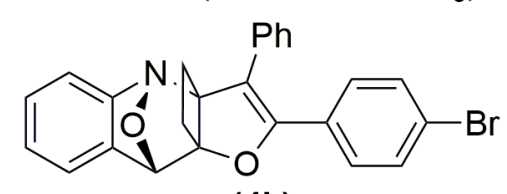

(4k) 

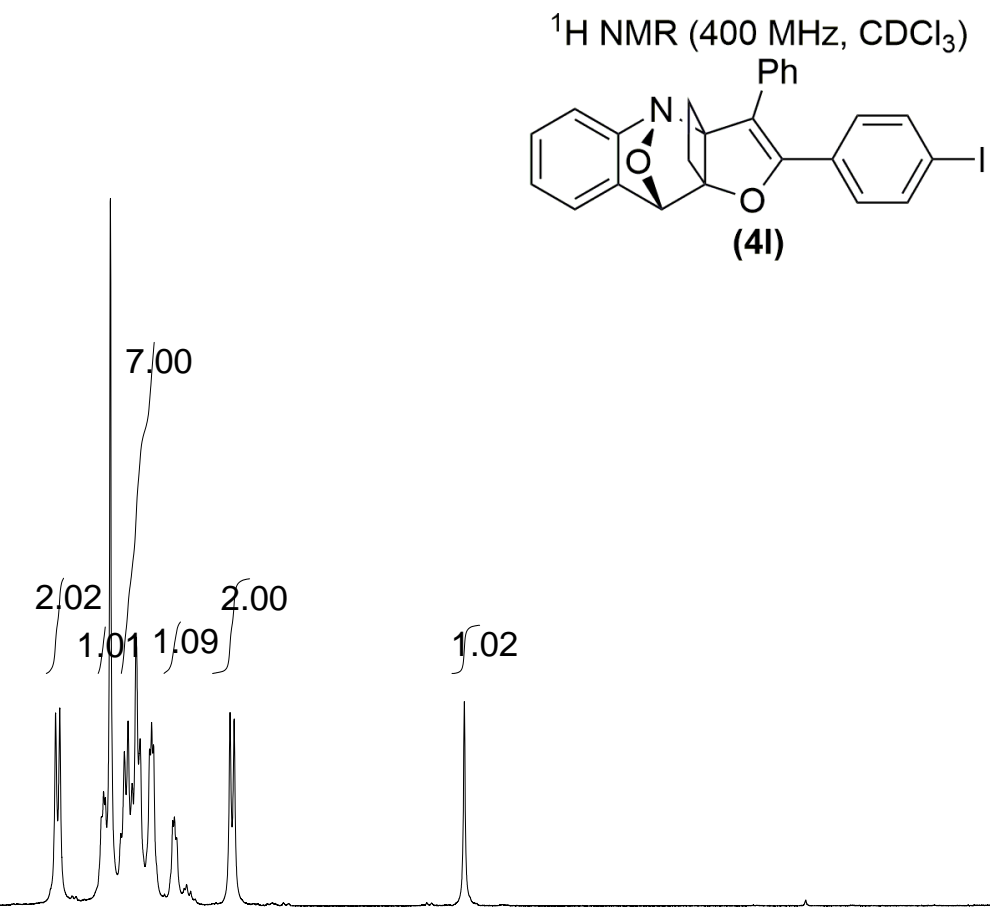

(4I) 


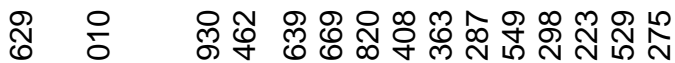

in

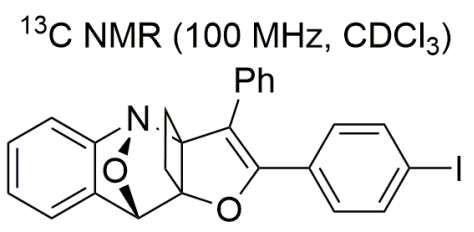

(4I) 
$\left.{ }^{13} \mathrm{C} \mathrm{NMR} \mathrm{(100} \mathrm{MHz,} \mathrm{CDCl}_{3}\right)$

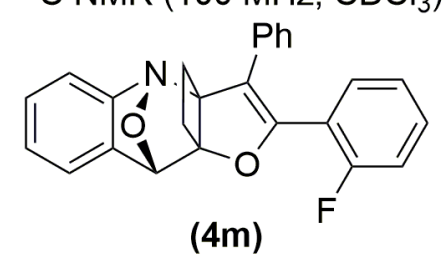




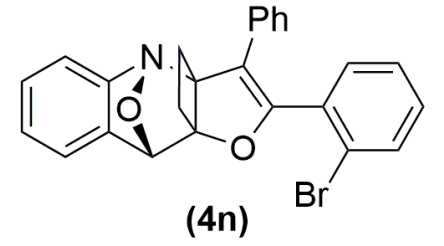


${ }^{13} \mathrm{C} \mathrm{NMR}\left(100 \mathrm{MHz}, \mathrm{CDCl}_{3}\right)$

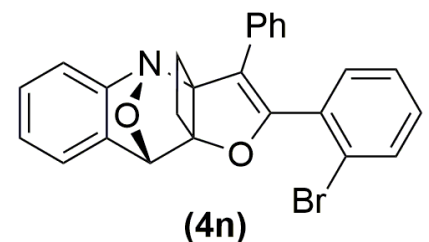

(4n) 


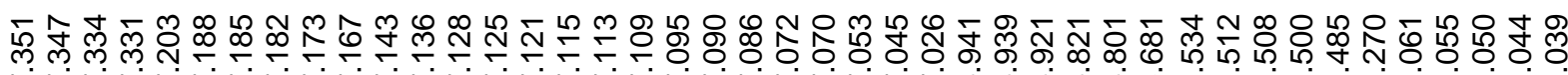

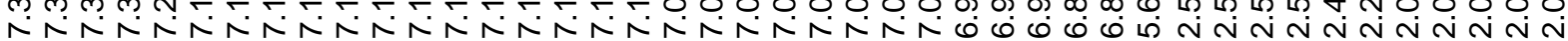
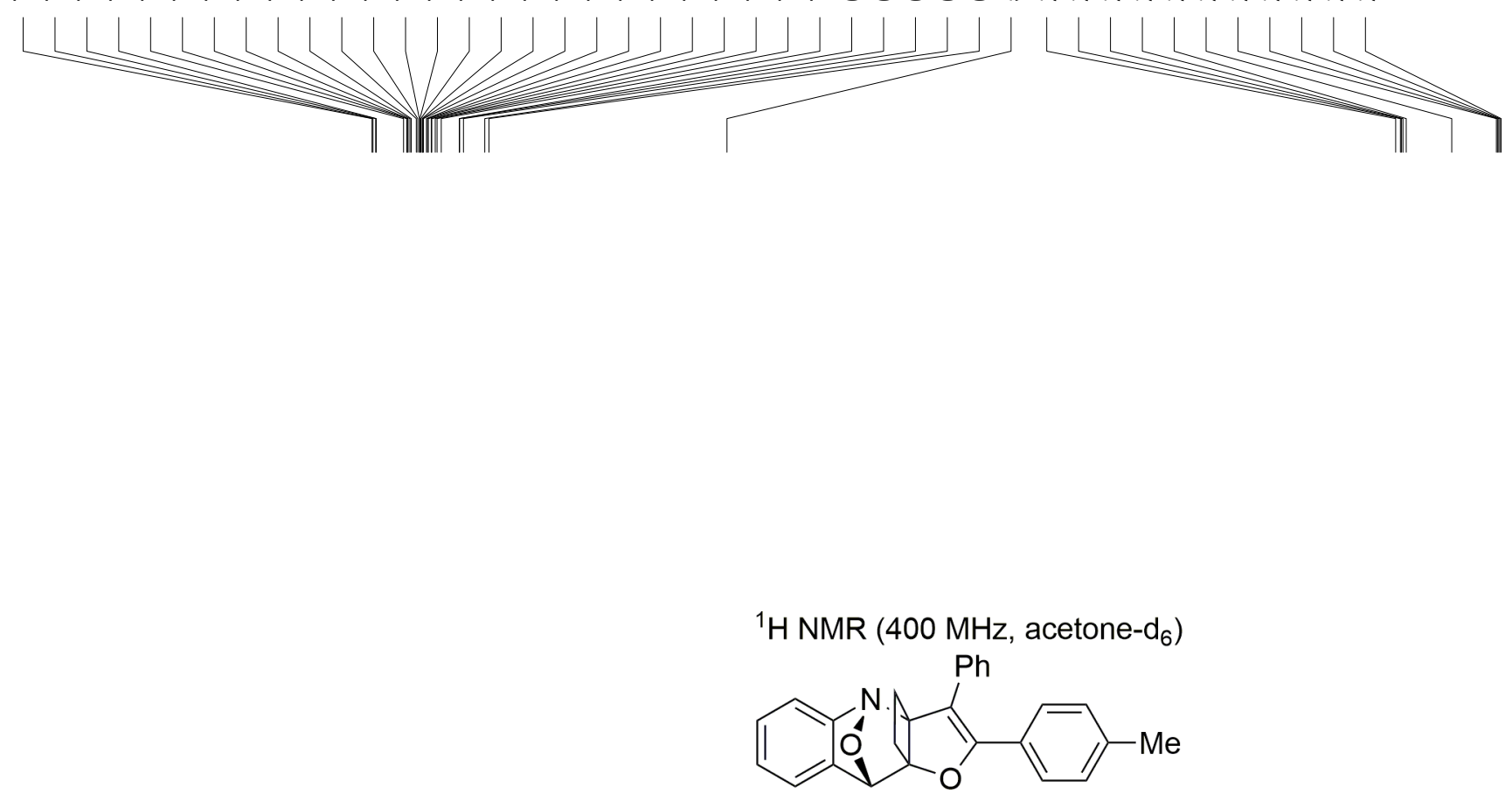

(40) 


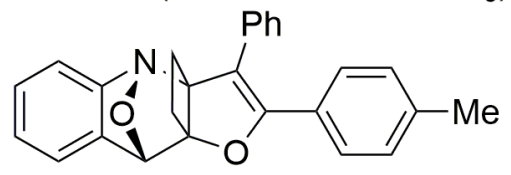

(40) 


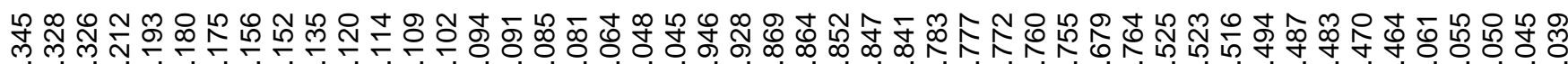

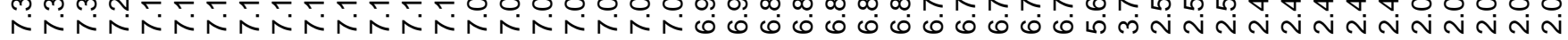
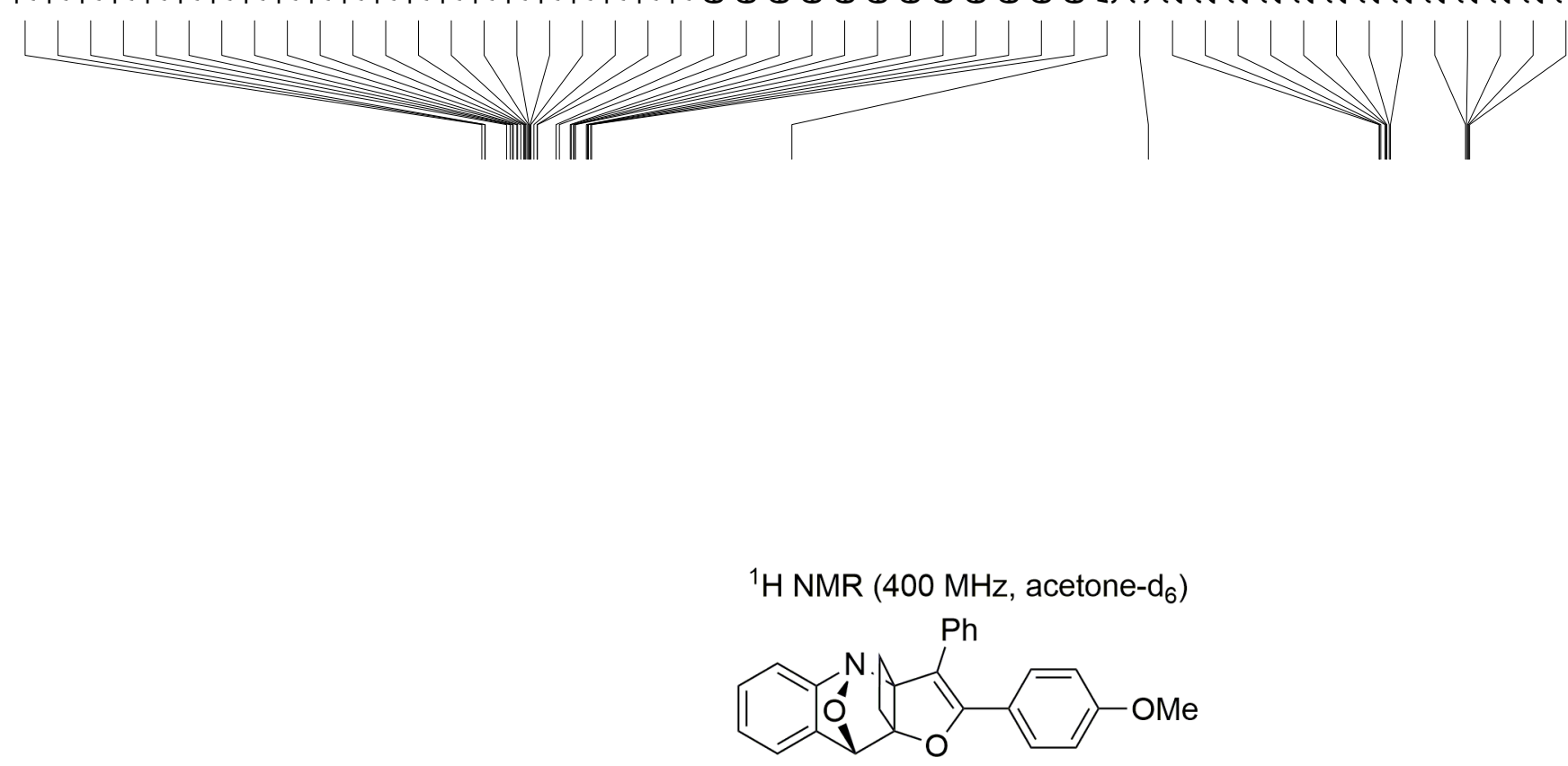

(4p) 
${ }^{13} \mathrm{C}$ NMR $\left(100 \mathrm{MHz}\right.$, acetone- $\left.\mathrm{d}_{6}\right)$

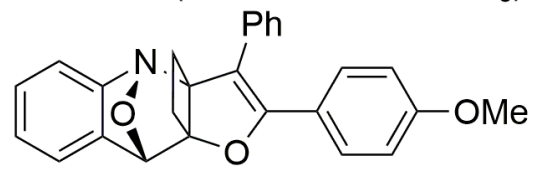

(4p) 


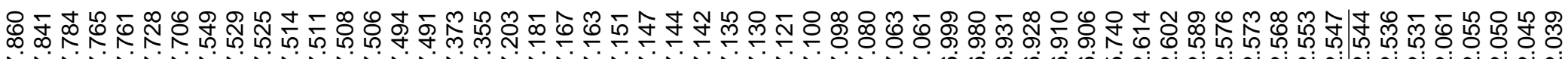

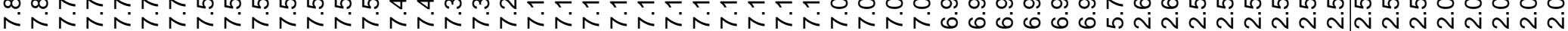

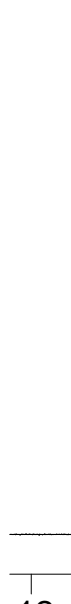

12
8

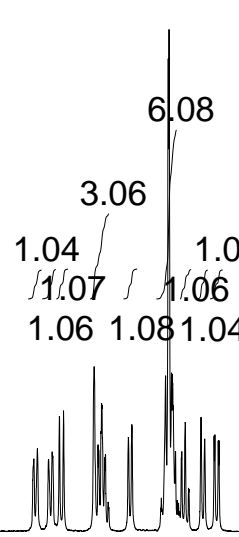

08 1.04

.06 1.04

8

${ }^{1} \mathrm{H}$ NMR (400 MHz, acetone- $\mathrm{d}_{6}$ )

(4q)

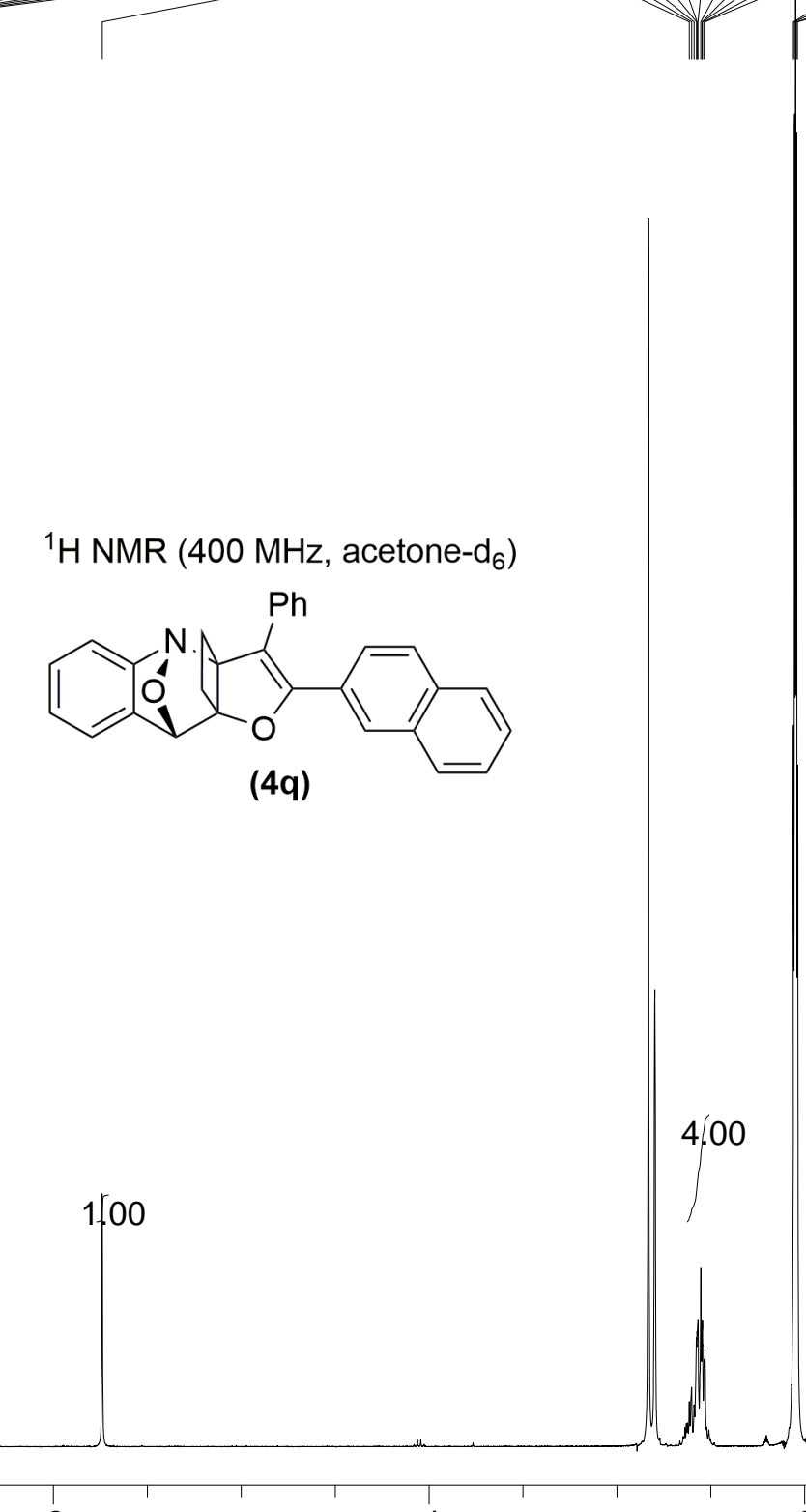

6

4

2

0 


\section{न न}

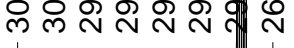

${ }^{13} \mathrm{C}$ NMR $\left(100 \mathrm{MHz}\right.$, acetone- $\left.\mathrm{d}_{6}\right)$

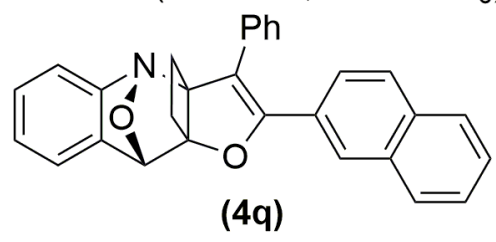



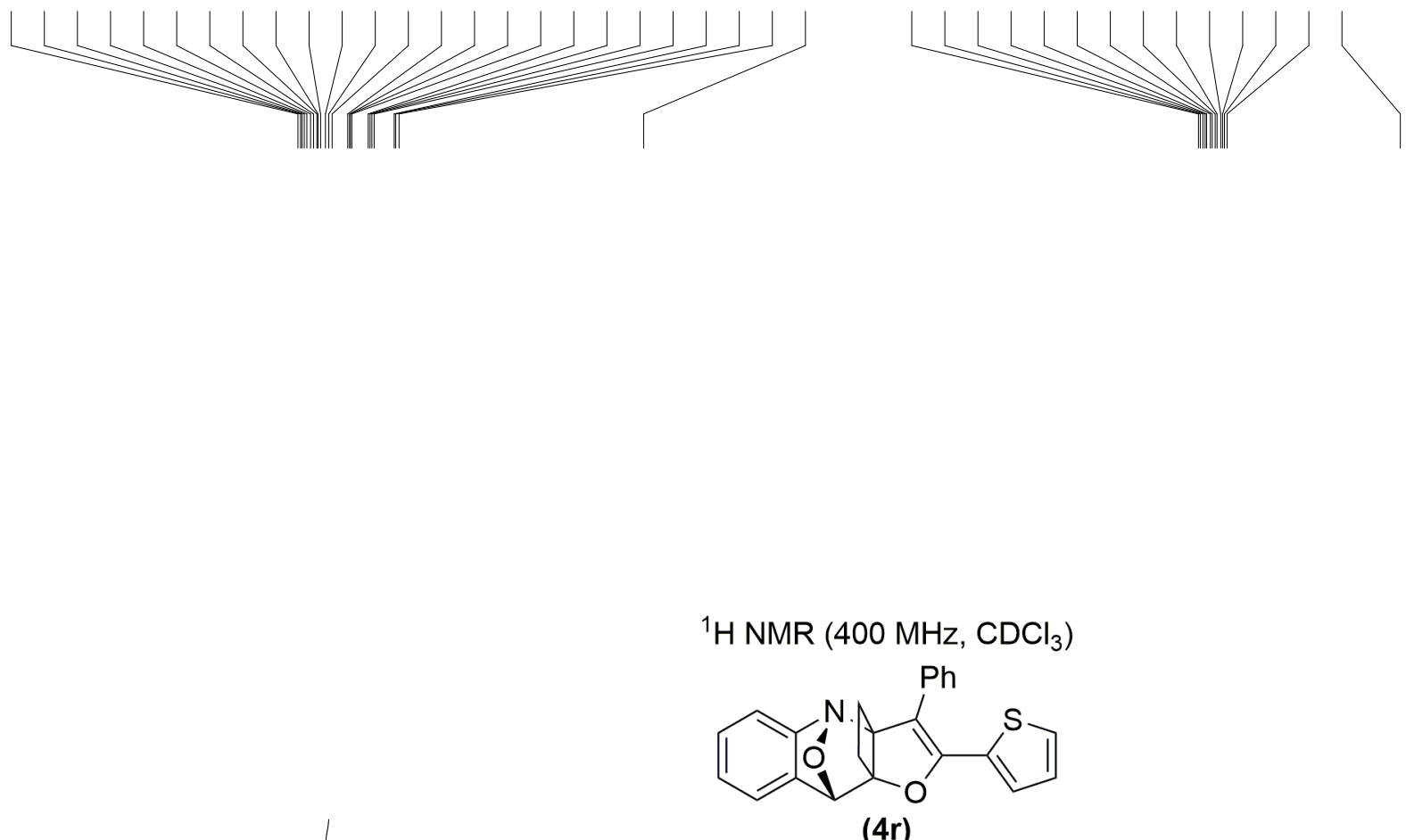
${ }^{13} \mathrm{C} \mathrm{NMR}\left(100 \mathrm{MHz}, \mathrm{CDCl}_{3}\right)$

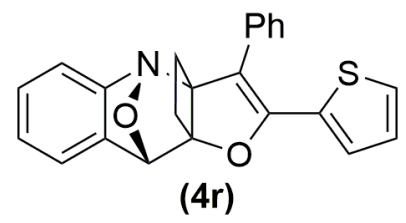




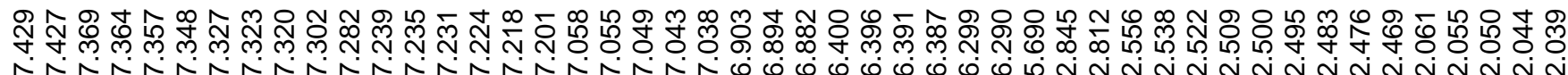

7ाI

${ }^{1} \mathrm{H}$ NMR (400 MHz, acetone- $d_{6}$ )

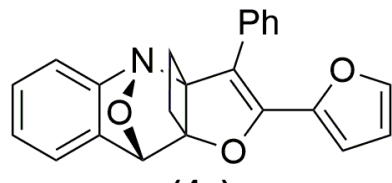

(4s)

5.09

$1.00021 .07 \quad 1.07 \quad 1.04$

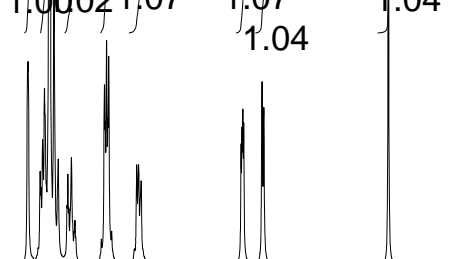




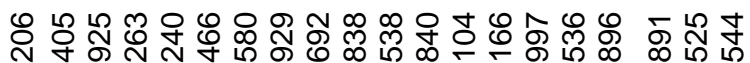

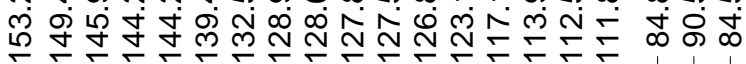

${ }^{13} \mathrm{C}$ NMR (100 MHz, acetone- $\left.d_{6}\right)$

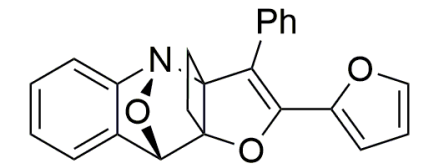

(4s)

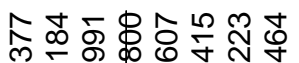

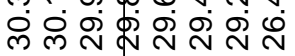




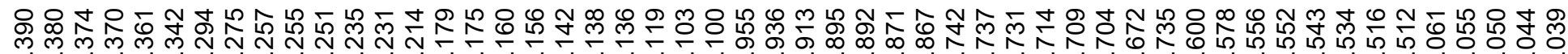

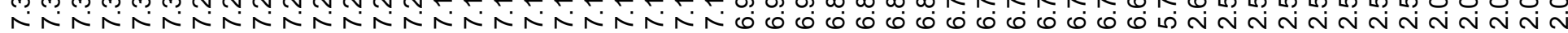

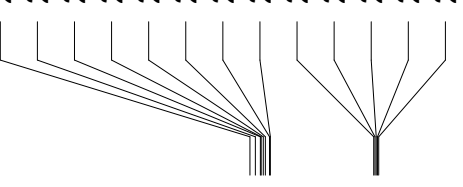

${ }^{1} \mathrm{H}$ NMR $\left(400 \mathrm{MHz}\right.$, acetone- $\left.\mathrm{d}_{6}\right)$

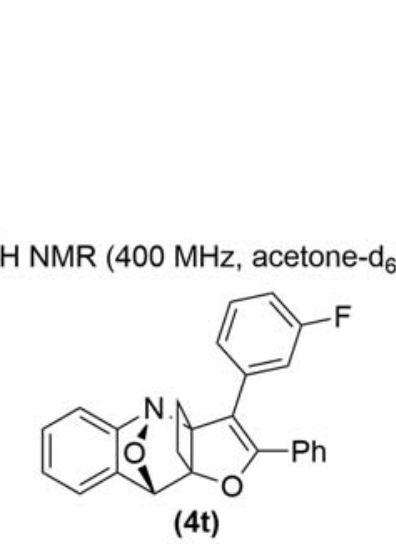

$(4 \mathrm{t})$

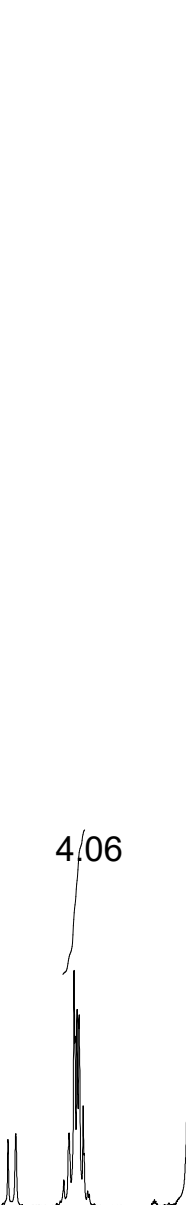



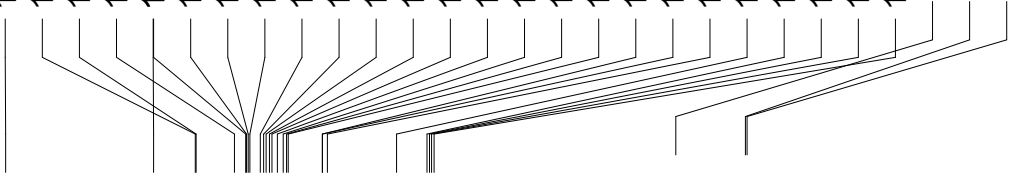

${ }^{13} \mathrm{C}$ NMR (100 MHz, acetone- $\left.\mathrm{d}_{6}\right)$

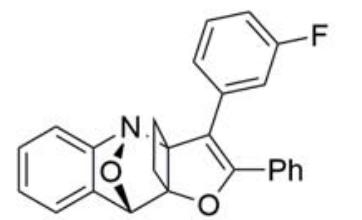

(4t) 


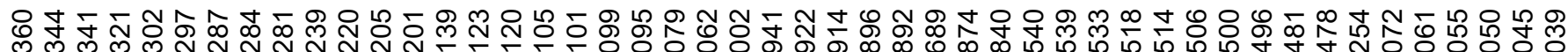

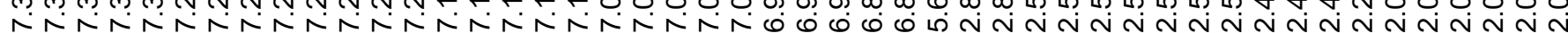
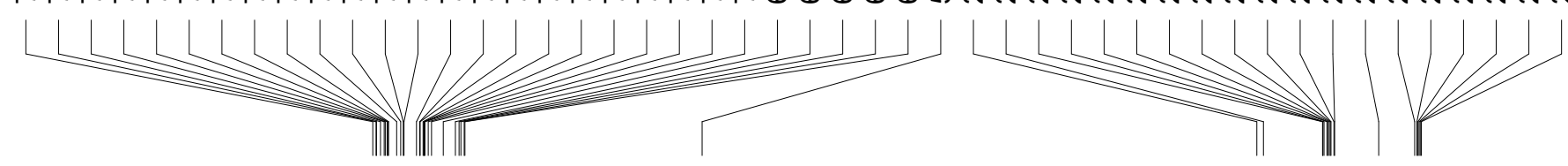

${ }^{1} \mathrm{H}$ NMR (400 MHz, acetone- $\mathrm{d}_{6}$ )
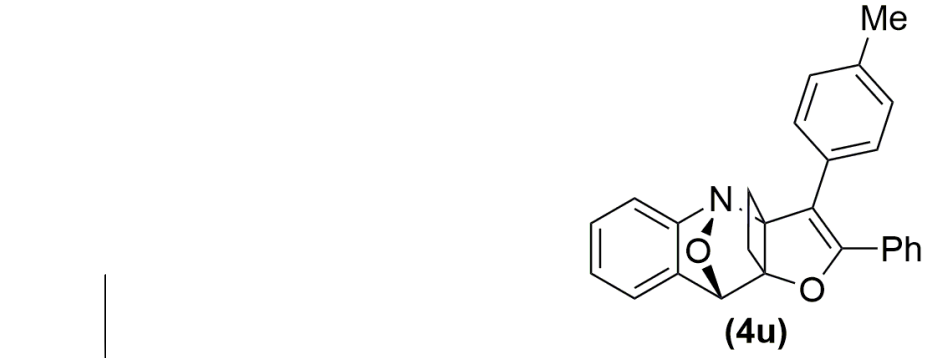

4.05

2.063 .09

2.08 .04

1..00

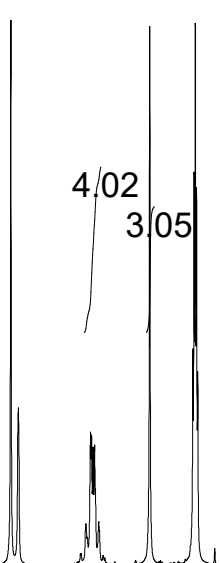

PPM 
${ }^{13} \mathrm{C}$ NMR $\left(100 \mathrm{MHz}\right.$, acetone- $\mathrm{d}_{6}$ )

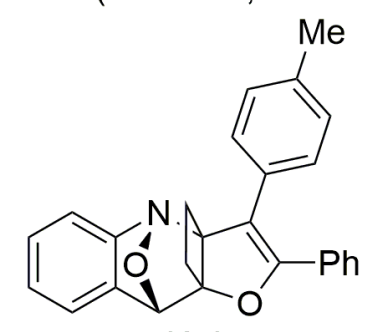

(4u) 


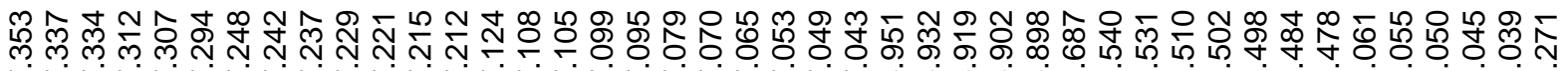

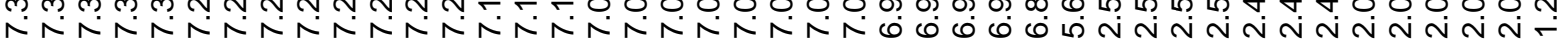
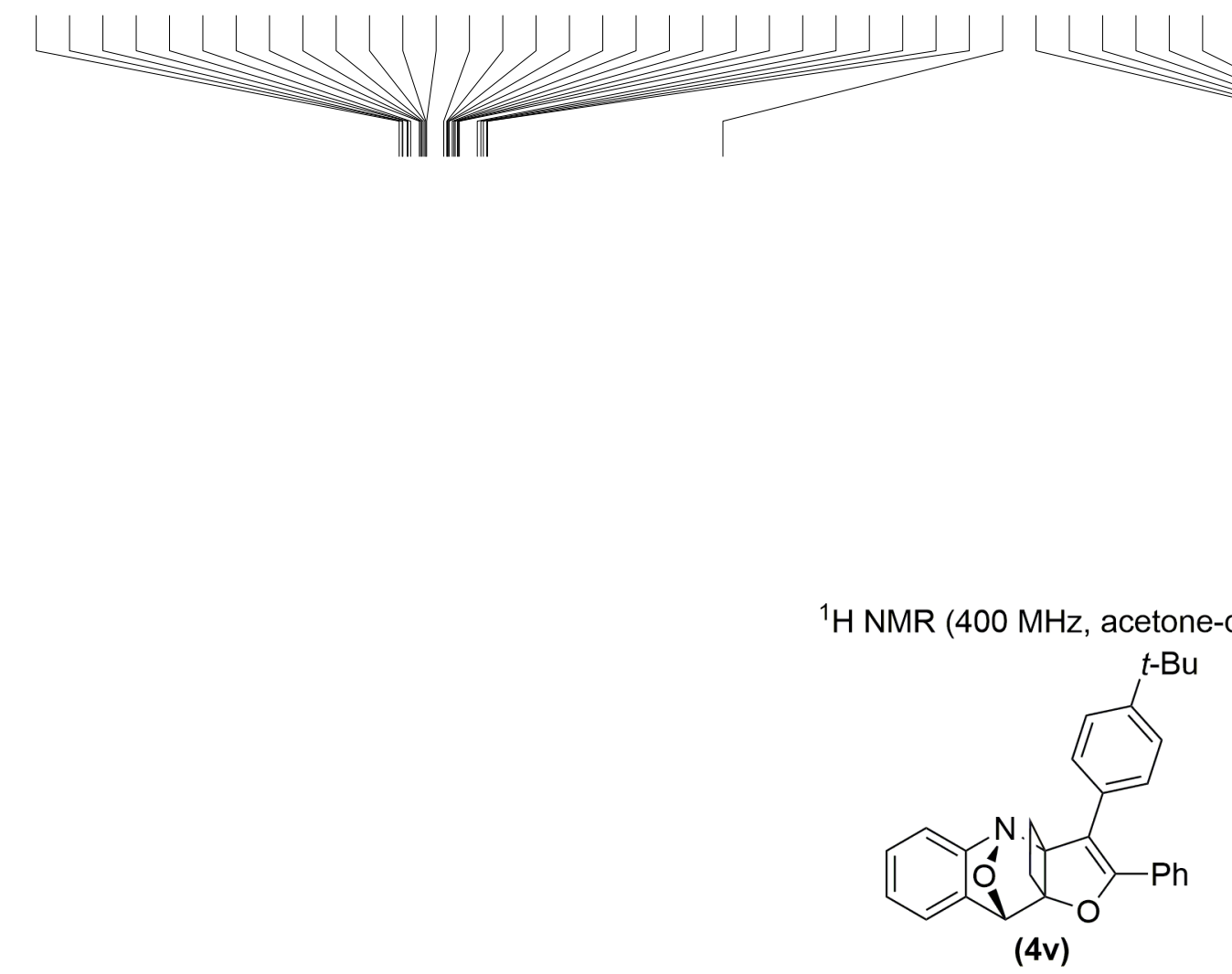

4.04

4.053 .06

2.05

1.00 
${ }^{13} \mathrm{C}$ NMR $\left(100 \mathrm{MHz}\right.$, acetone- $\left.\mathrm{d}_{6}\right)$

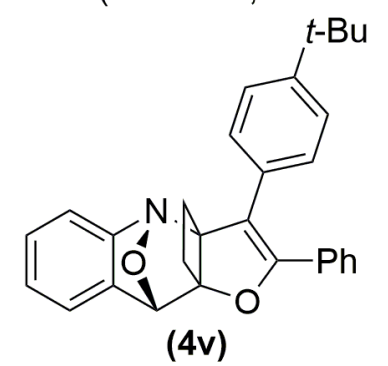

$t$-Bu 


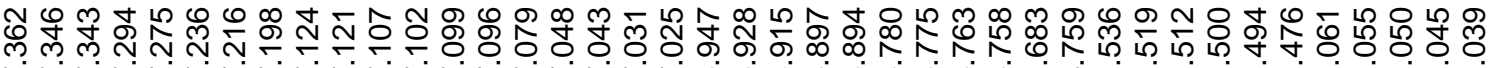

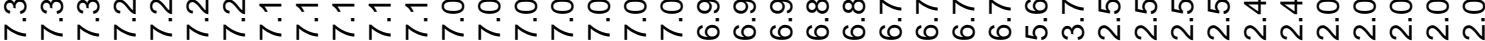
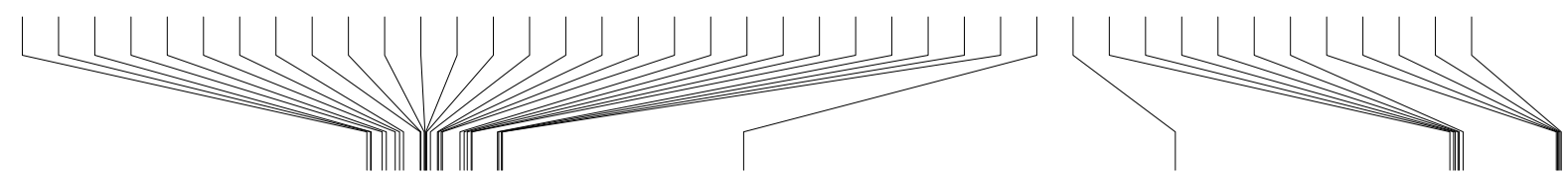

${ }^{1} \mathrm{H}$ NMR (400 MHz, acetone- $\mathrm{d}_{6}$ )

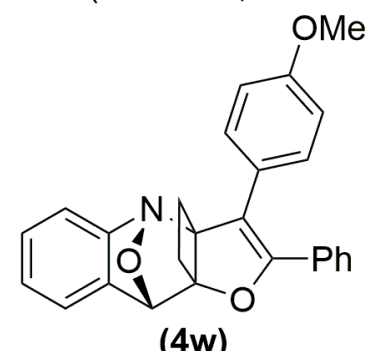

(4w)

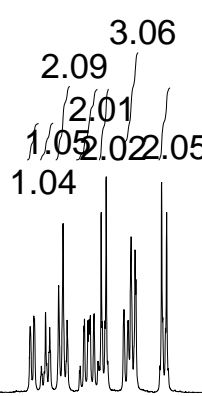

1.00 
${ }^{13} \mathrm{C}$ NMR $\left(100 \mathrm{MHz}\right.$, acetone- $\left.\mathrm{d}_{6}\right)$

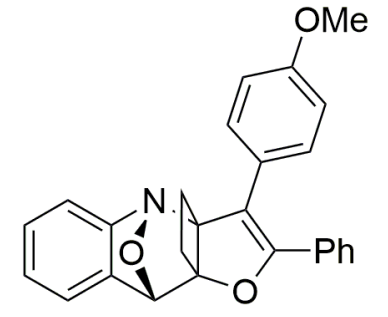

(4w) 


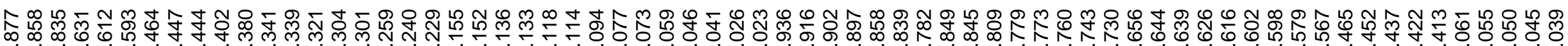

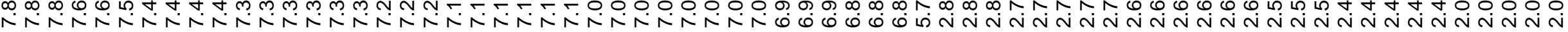

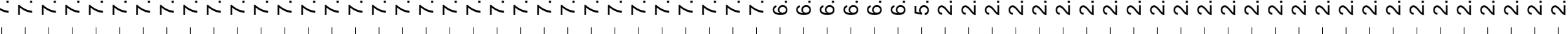

${ }^{1} \mathrm{H}$ NMR (400 MHz, acetone- $\mathrm{d}_{6}$ )

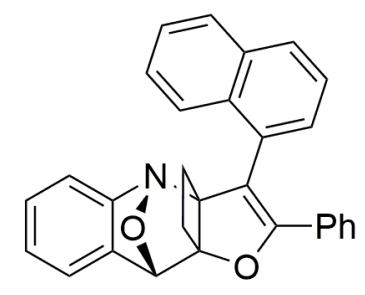

$(4 \mathrm{x})$

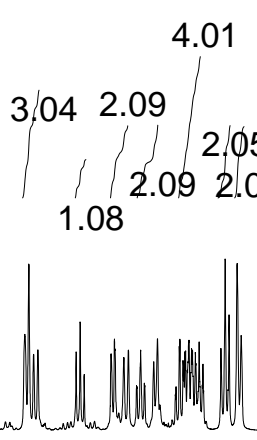

4.01

2.05

1.00

1.00

1.07 
${ }^{13} \mathrm{C}$ NMR $\left(100 \mathrm{MHz}\right.$, acetone- $\left.\mathrm{d}_{6}\right)$

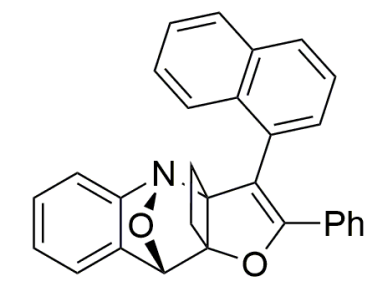

(4x) 


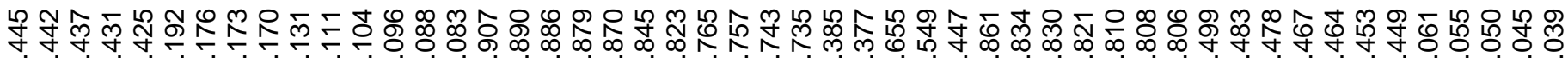

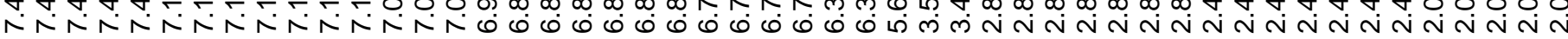
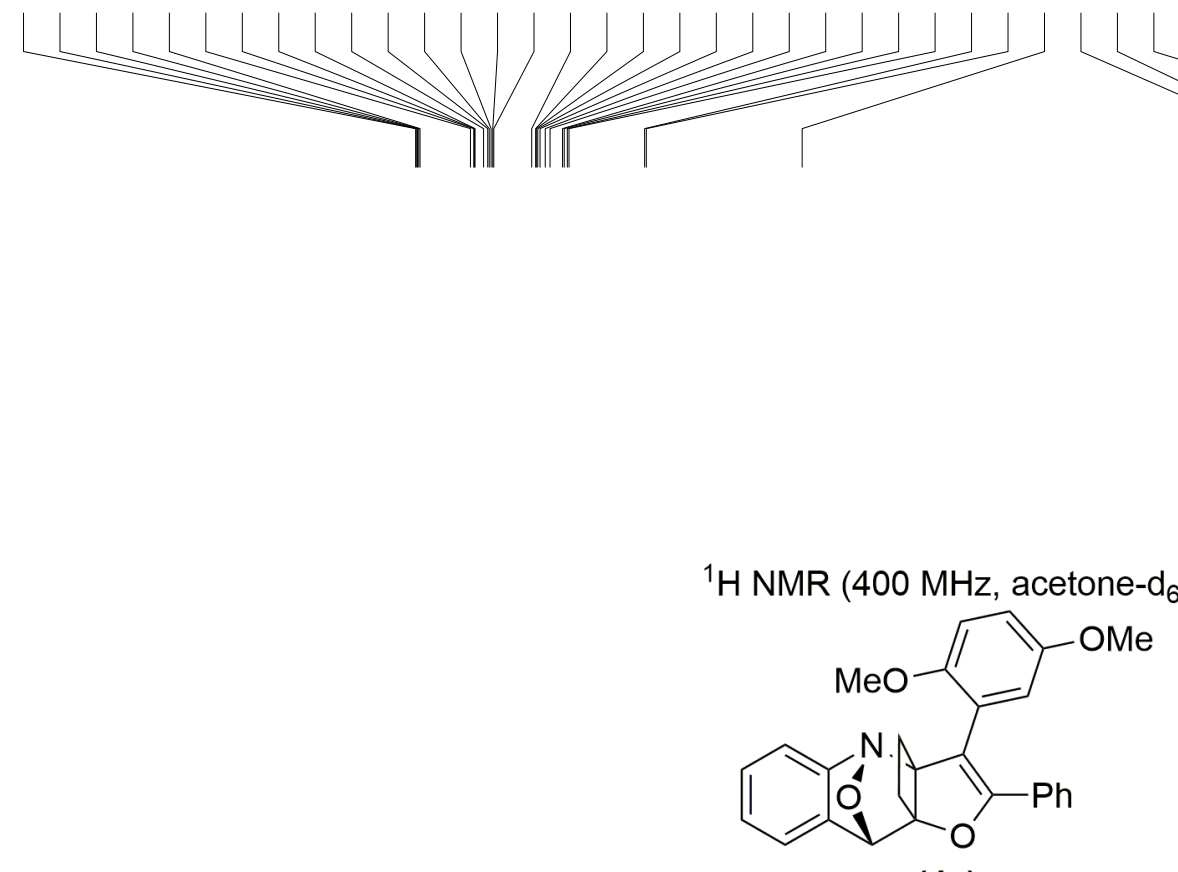

(4y)

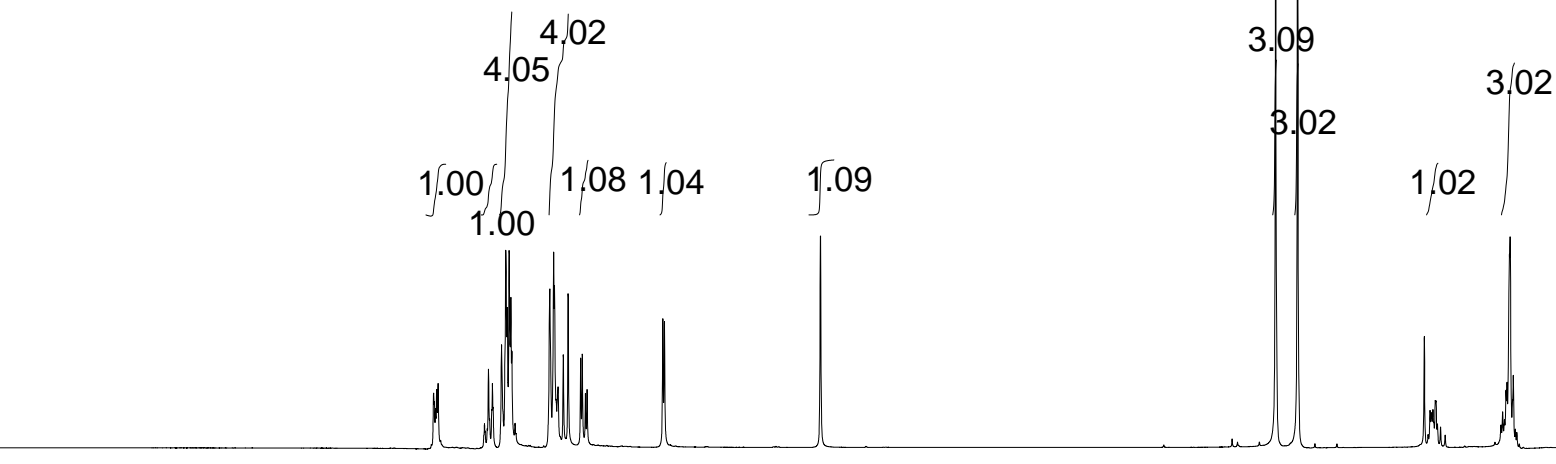

$$
3.09
$$




\section{${ }^{13} \mathrm{C}$ NMR $\left(100 \mathrm{MHz}\right.$, acetone- $\left.\mathrm{d}_{6}\right)$}

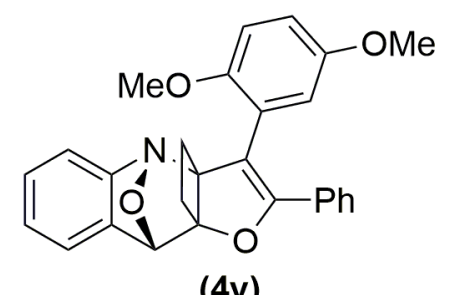

(4y) 


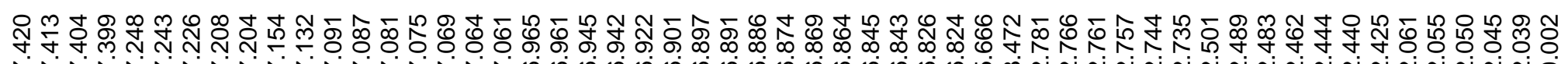

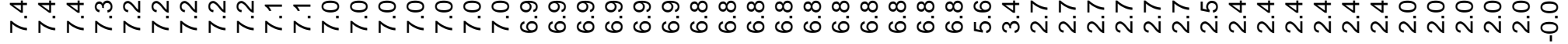

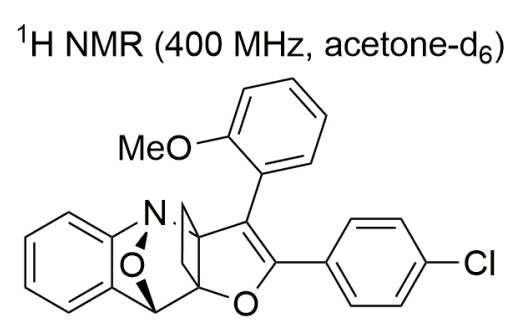

(4z) 
${ }^{13} \mathrm{C}$ NMR $\left(100 \mathrm{MHz}\right.$, acetone- $\left.\mathrm{d}_{6}\right)$

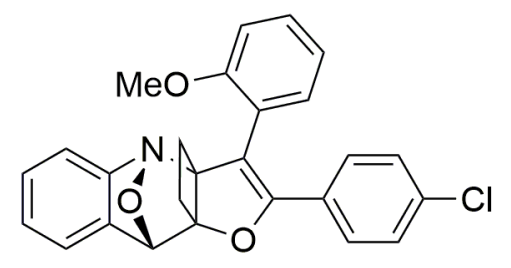

(4z) 


\section{${ }^{1} \mathrm{H} \mathrm{NMR}\left(400 \mathrm{MHz}, \mathrm{CDCl}_{3}\right)$}

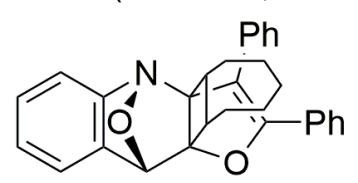

(4aa)
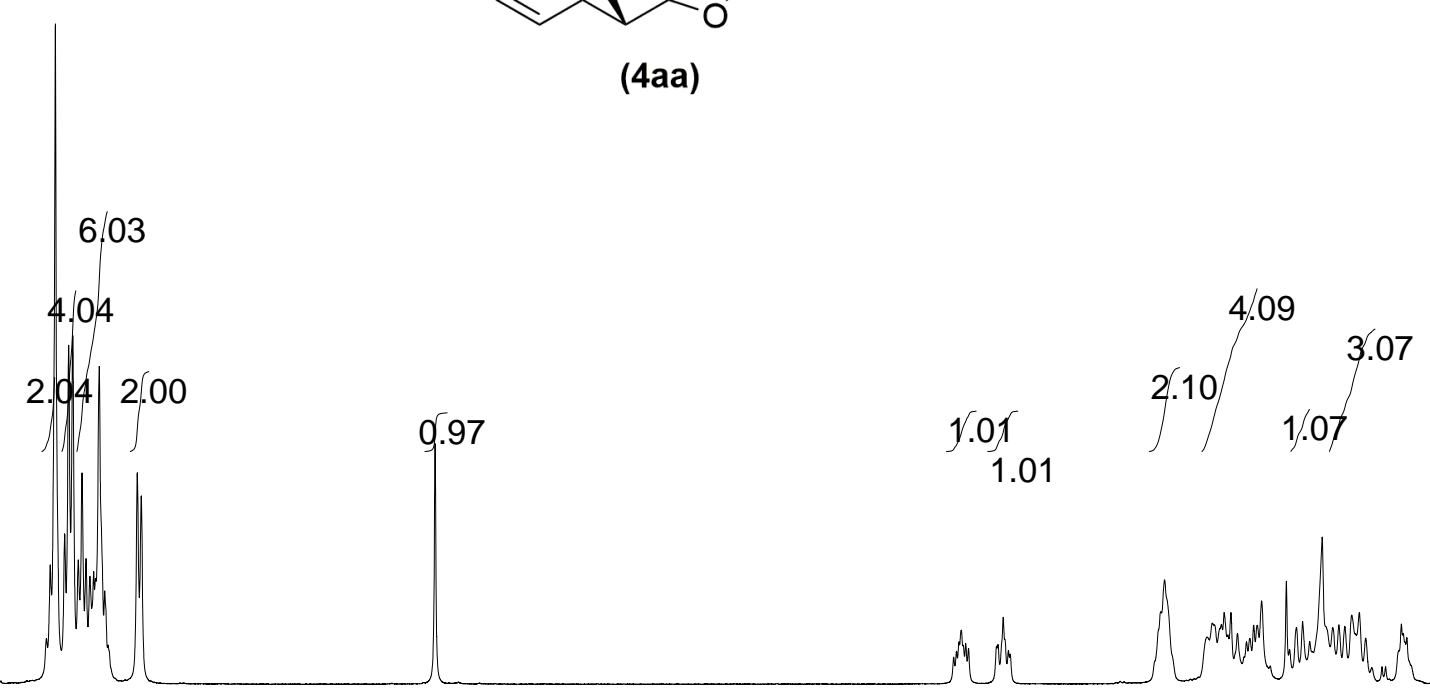


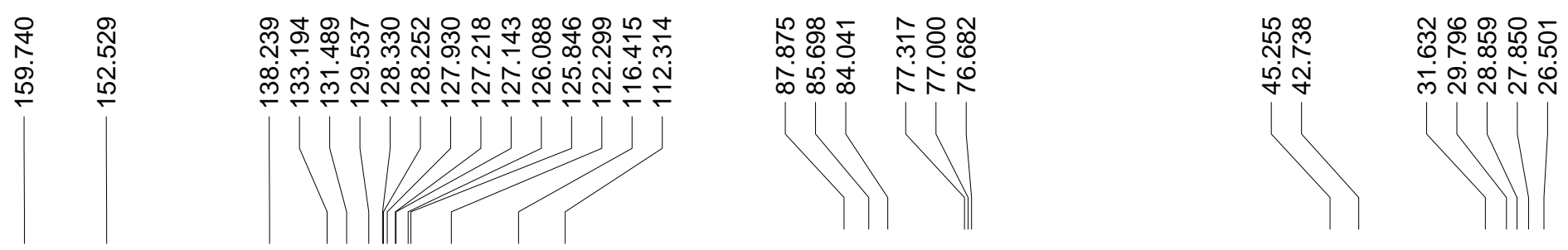

${ }^{13} \mathrm{C} \mathrm{NMR}\left(100 \mathrm{MHz}, \mathrm{CDCl}_{3}\right)$

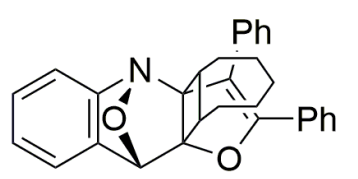

(4aa) 


\section{${ }^{1} \mathrm{H}$ NMR $\left(400 \mathrm{MHz}, \mathrm{CDCl}_{3}\right)$}
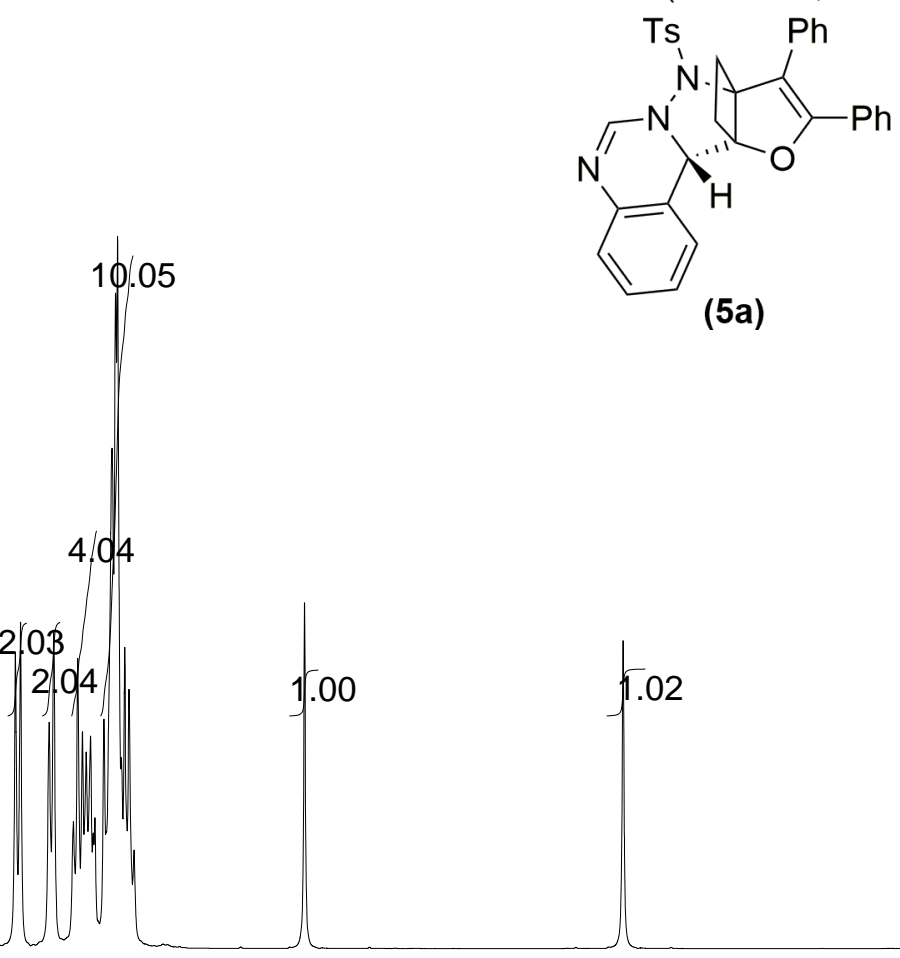

1.011 .021 .02 .06 
${ }^{13} \mathrm{C}$ NMR (100 MHz, $\mathrm{CDCl}_{3}$ )

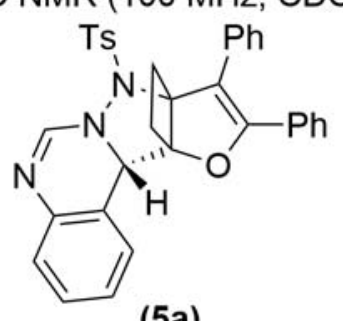

(5a) 

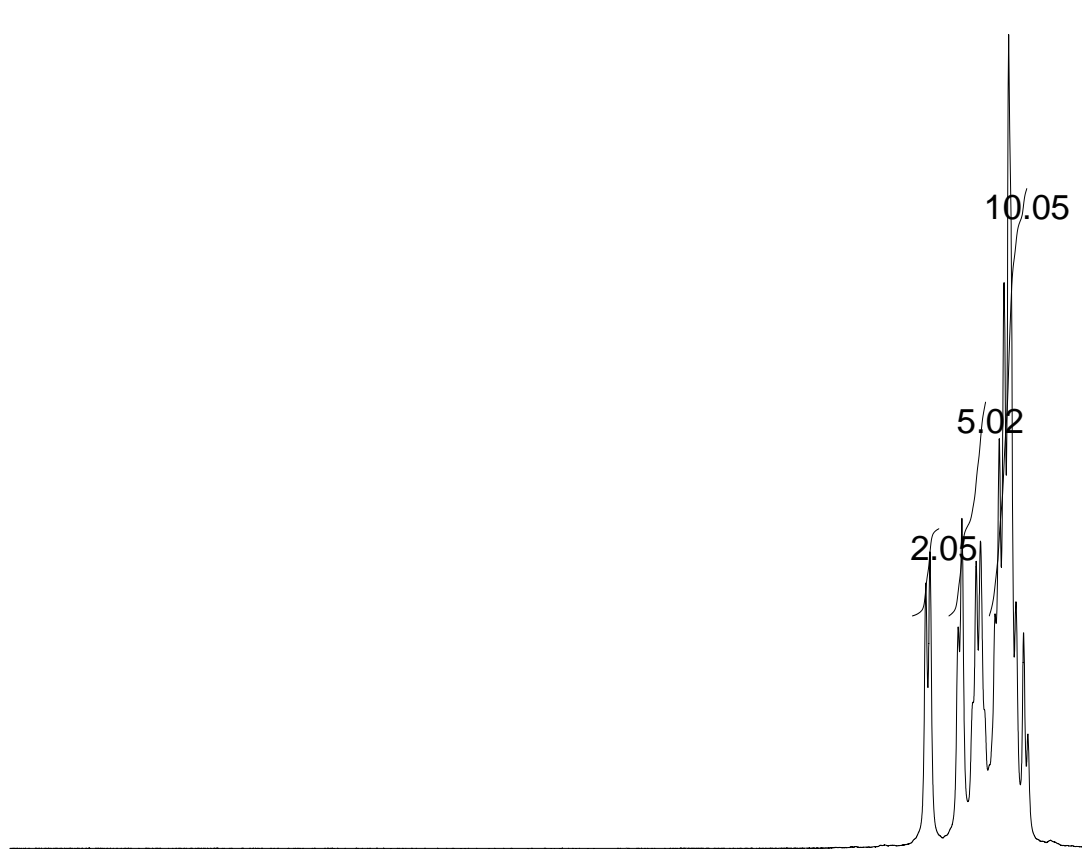

(5b)

\section{${ }^{1} \mathrm{H} \mathrm{NMR}\left(400 \mathrm{MHz}, \mathrm{CDCl}_{3}\right)$}

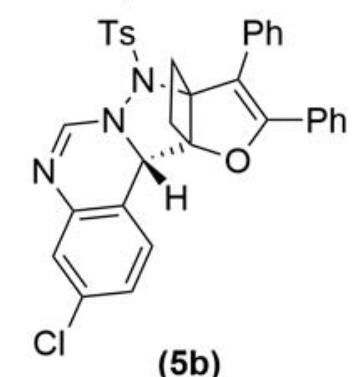

$$
3.05
$$

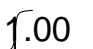

1.02

1.031 .011 .0409 


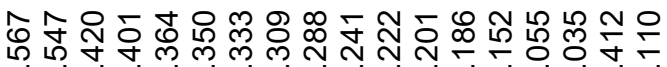

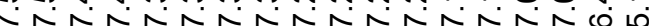

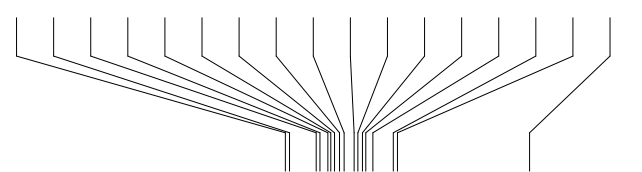

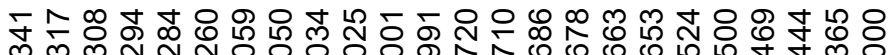

అ

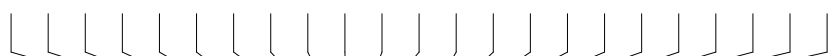

${ }^{1} \mathrm{H} \mathrm{NMR}\left(400 \mathrm{MHz}, \mathrm{CDCl}_{3}\right)$

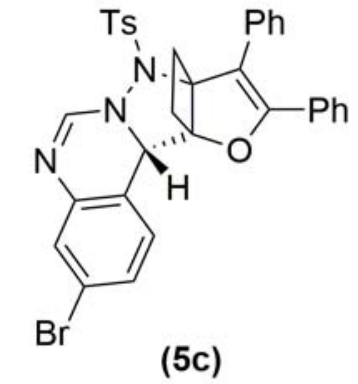

$$
77.05
$$

(5c)

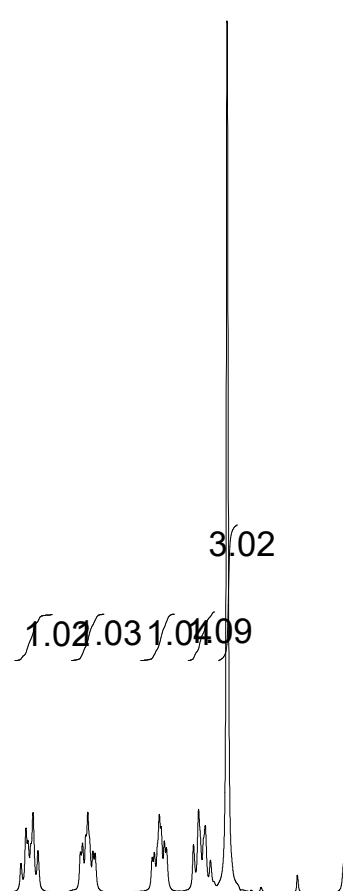


${ }^{13} \mathrm{C}$ NMR $\left(100 \mathrm{MHz}, \mathrm{CDCl}_{3}\right)$

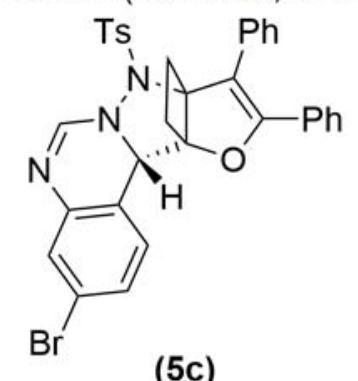

(5c) 


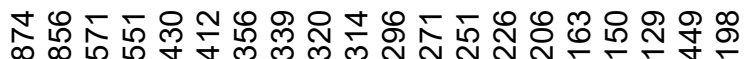

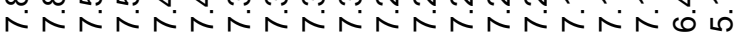
LLLLLL

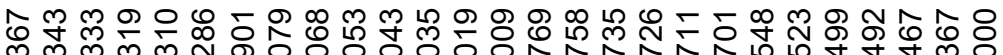
అ

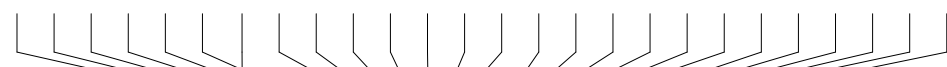

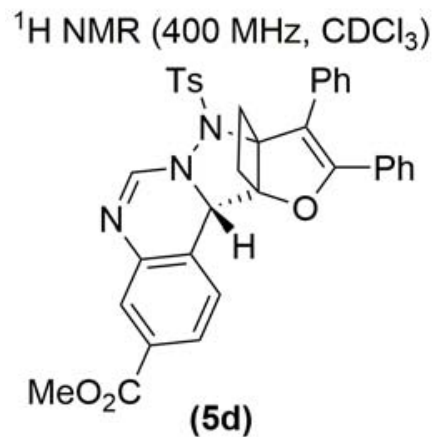

(5d) 
${ }^{13} \mathrm{C}$ NMR $\left(100 \mathrm{MHz}, \mathrm{CDCl}_{3}\right)$

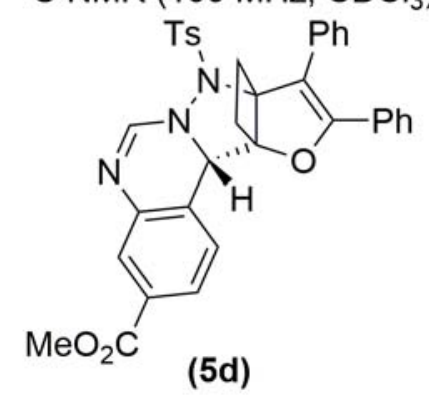




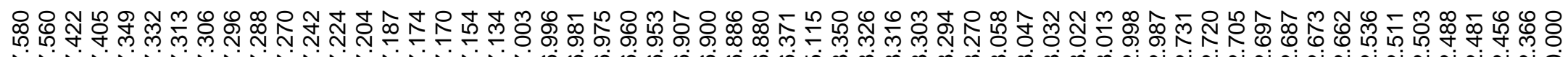

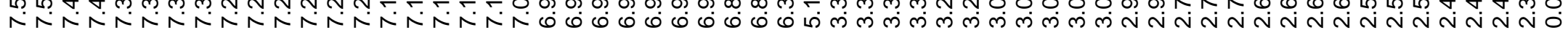

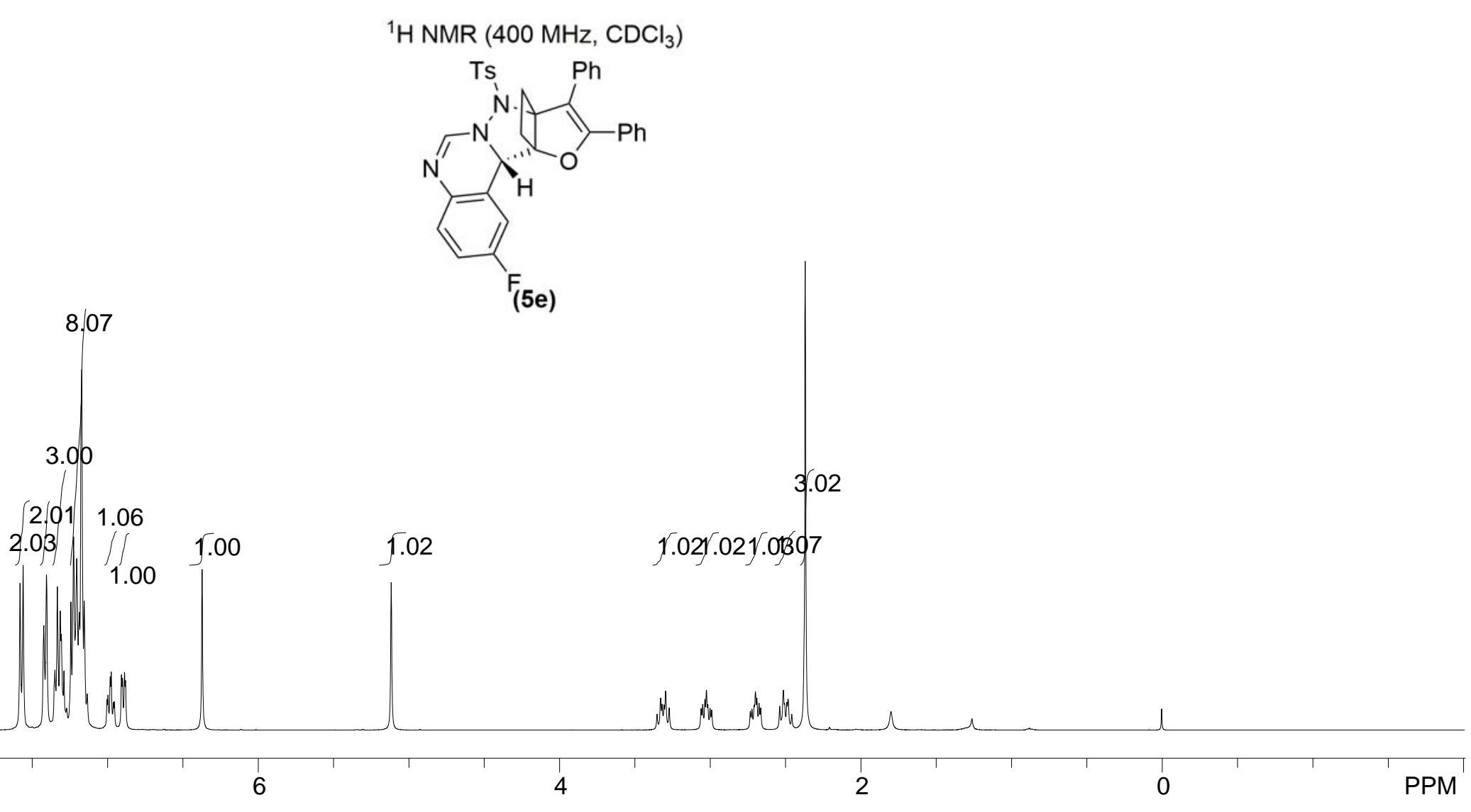


${ }^{13} \mathrm{C}$ NMR (100 MHz, $\mathrm{CDCl}_{3}$ )

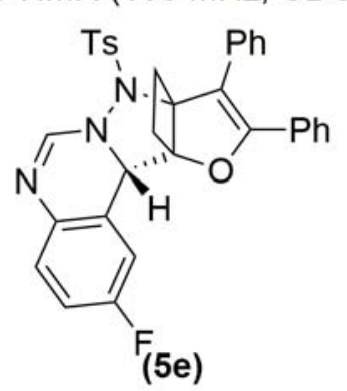


${ }^{13} \mathrm{C}$ NMR $\left(100 \mathrm{MHz}, \mathrm{CDCl}_{3}\right)$

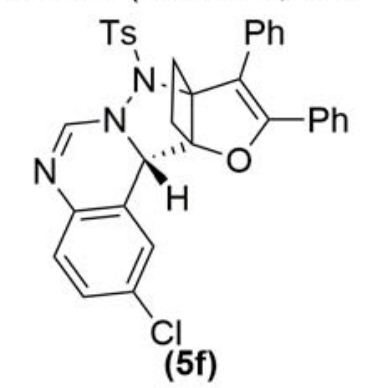




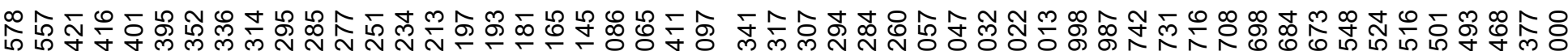

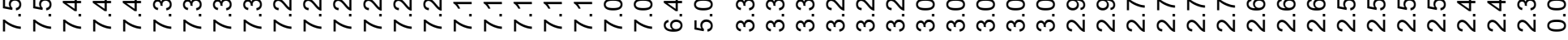

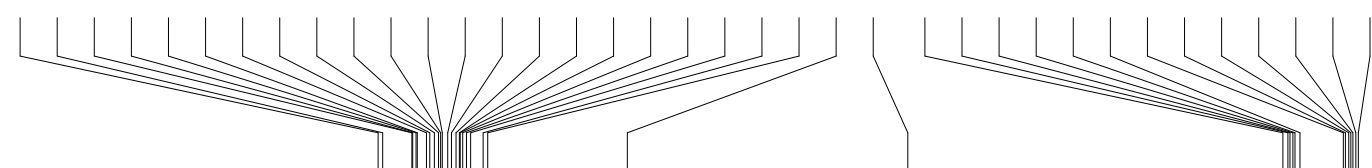

${ }^{1} \mathrm{H} \mathrm{NMR}\left(400 \mathrm{MHz}, \mathrm{CDCl}_{3}\right)$

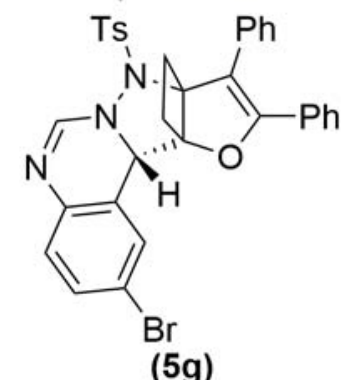




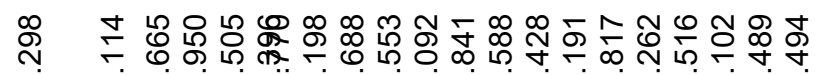

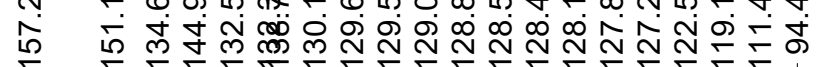

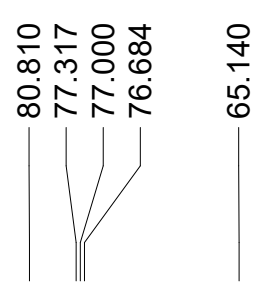

${ }^{13} \mathrm{C}$ NMR (100 MHz, $\mathrm{CDCl}_{3}$ )

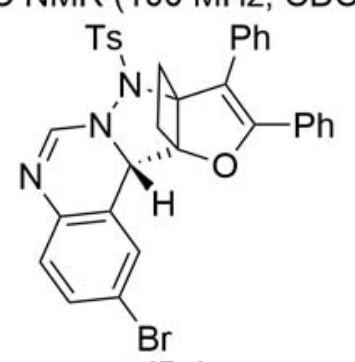

(5g)

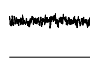




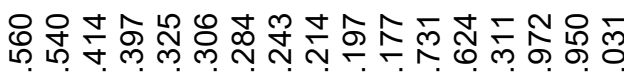

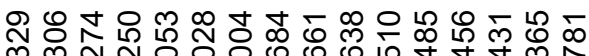

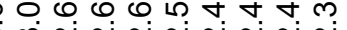

\section{${ }^{1} \mathrm{H}$ NMR $\left(400 \mathrm{MHz}, \mathrm{CDCl}_{3}\right)$}

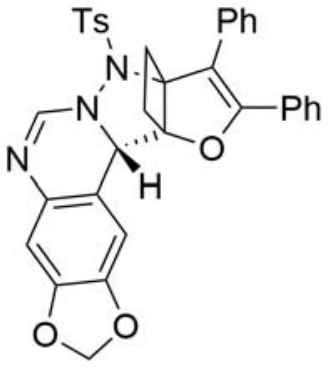

(5h)
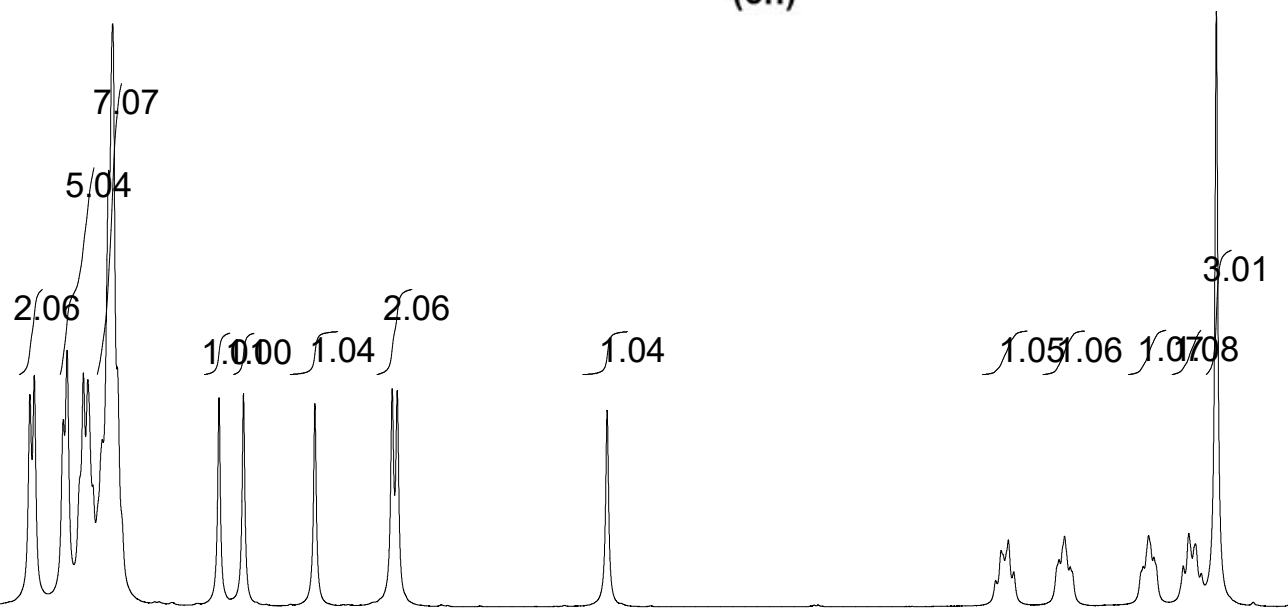

4

2 


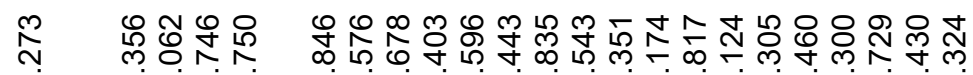

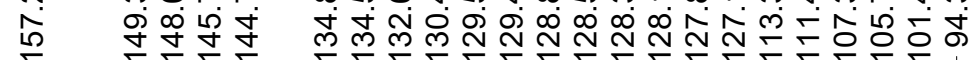

${ }^{13} \mathrm{C}$ NMR $\left(100 \mathrm{MHz}, \mathrm{CDCl}_{3}\right.$ )

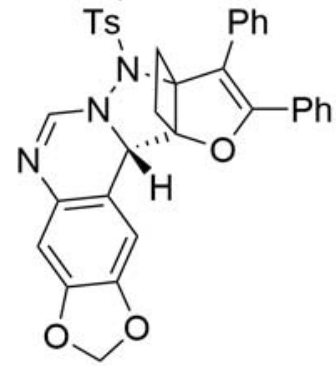

(5h) 

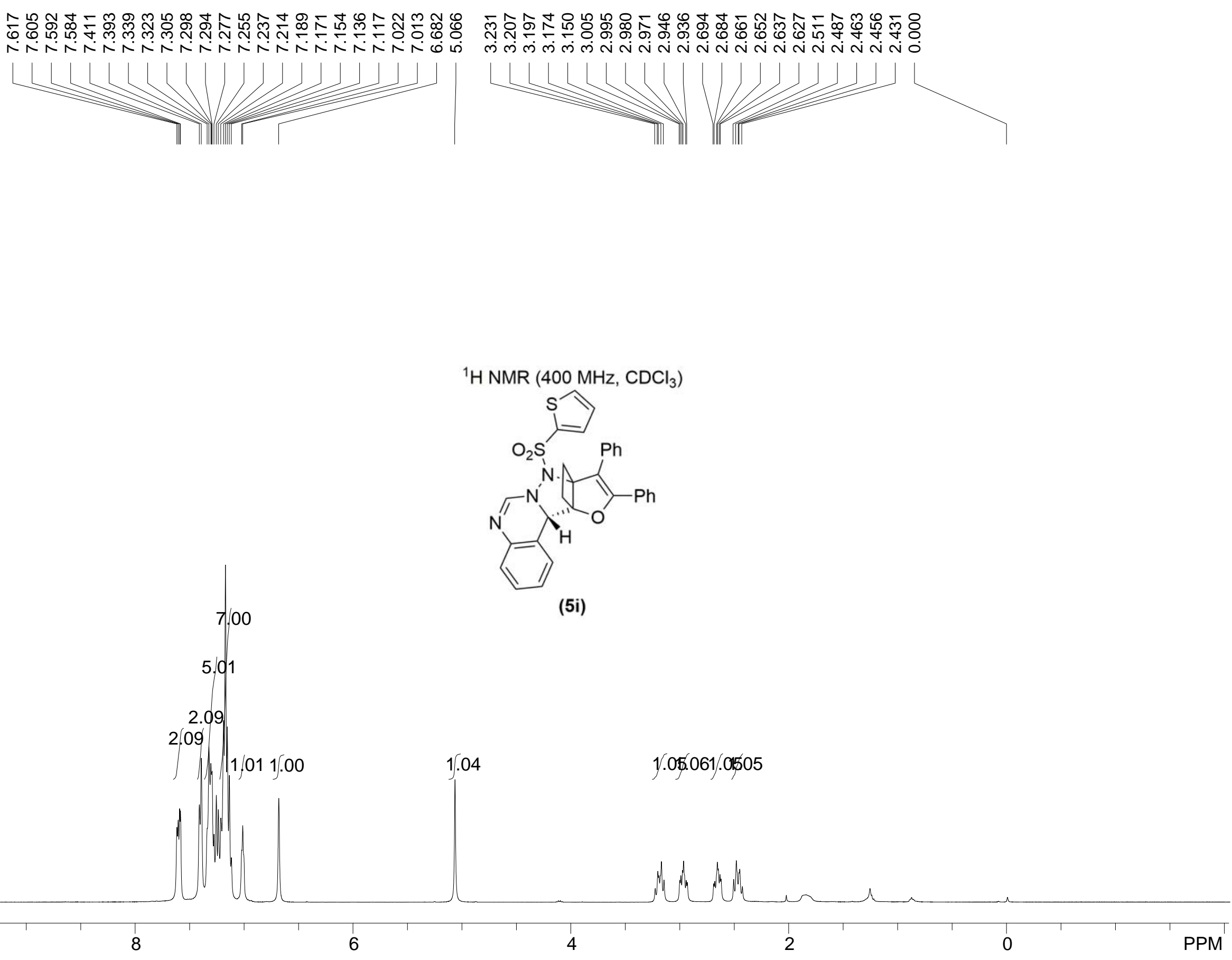
${ }^{13} \mathrm{C}$ NMR $\left(100 \mathrm{MHz}, \mathrm{CDCl}_{3}\right)$

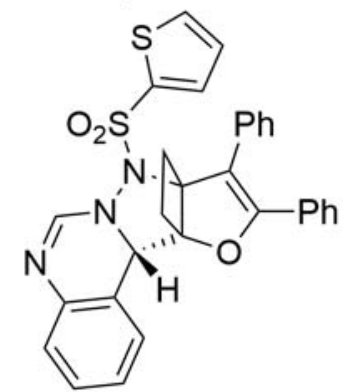

(5i) 


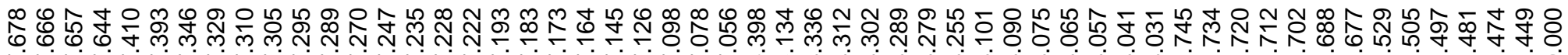

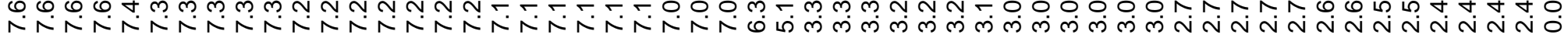
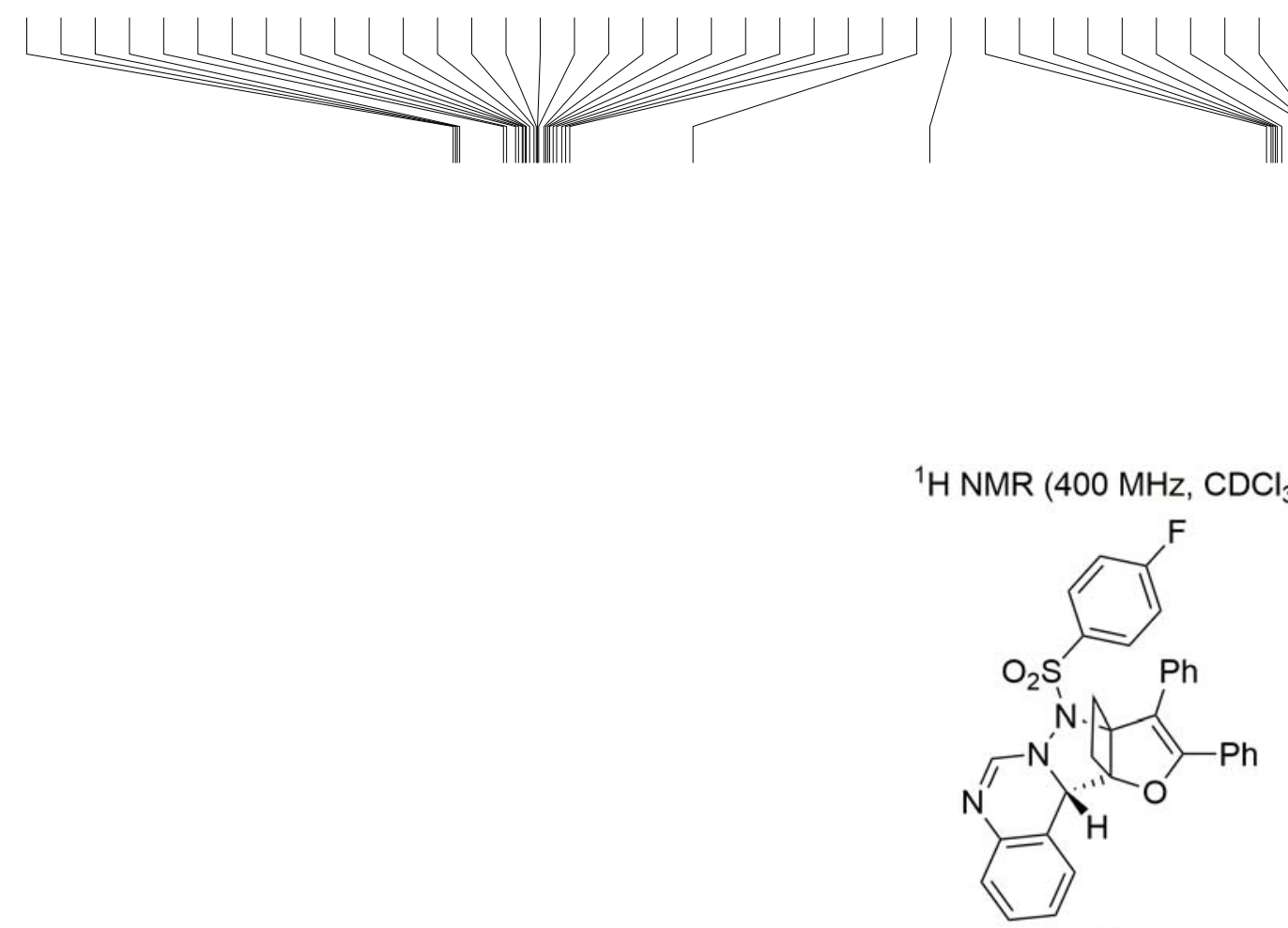

8.08

$(5 j)$ 


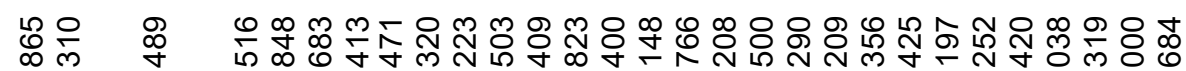

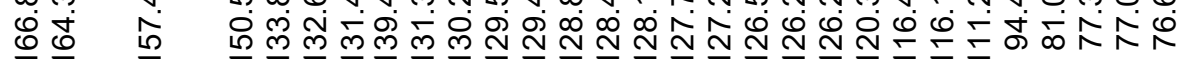

${ }^{13} \mathrm{C} \mathrm{NMR}\left(100 \mathrm{MHz}, \mathrm{CDCl}_{3}\right)$

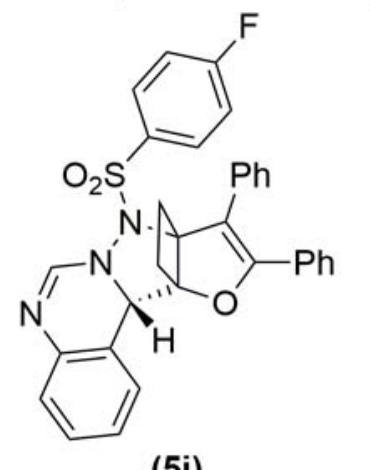

(5j) 


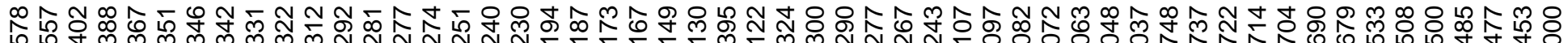

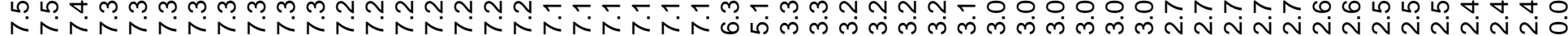
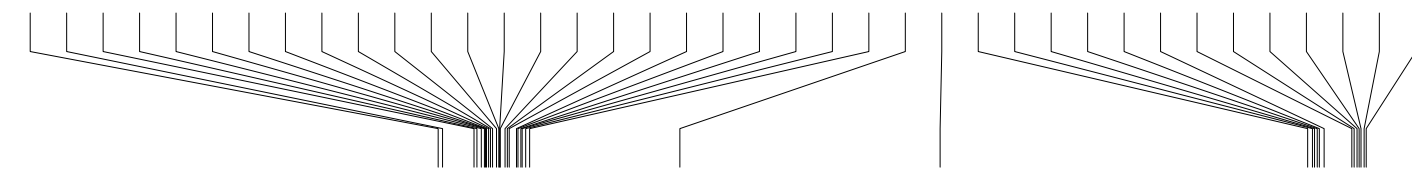

\section{${ }^{1} \mathrm{H} \mathrm{NMR}\left(400 \mathrm{MHz}, \mathrm{CDCl}_{3}\right)$}

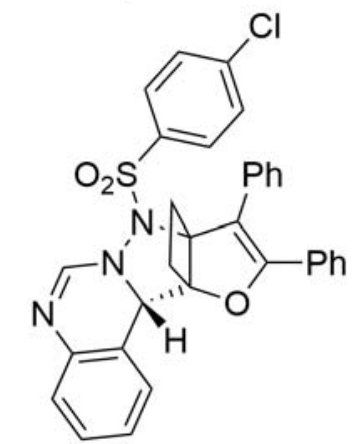

$(5 k)$

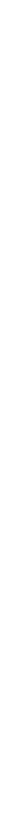


${ }^{13} \mathrm{CNMR}\left(100 \mathrm{MHz}, \mathrm{CDCl}_{3}\right)$

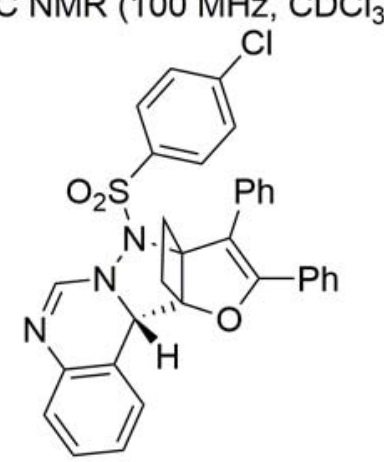

(5k) 


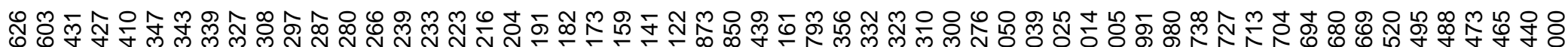

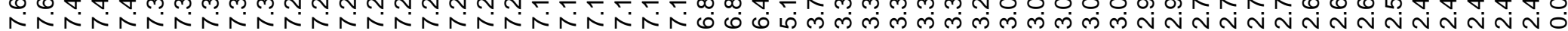
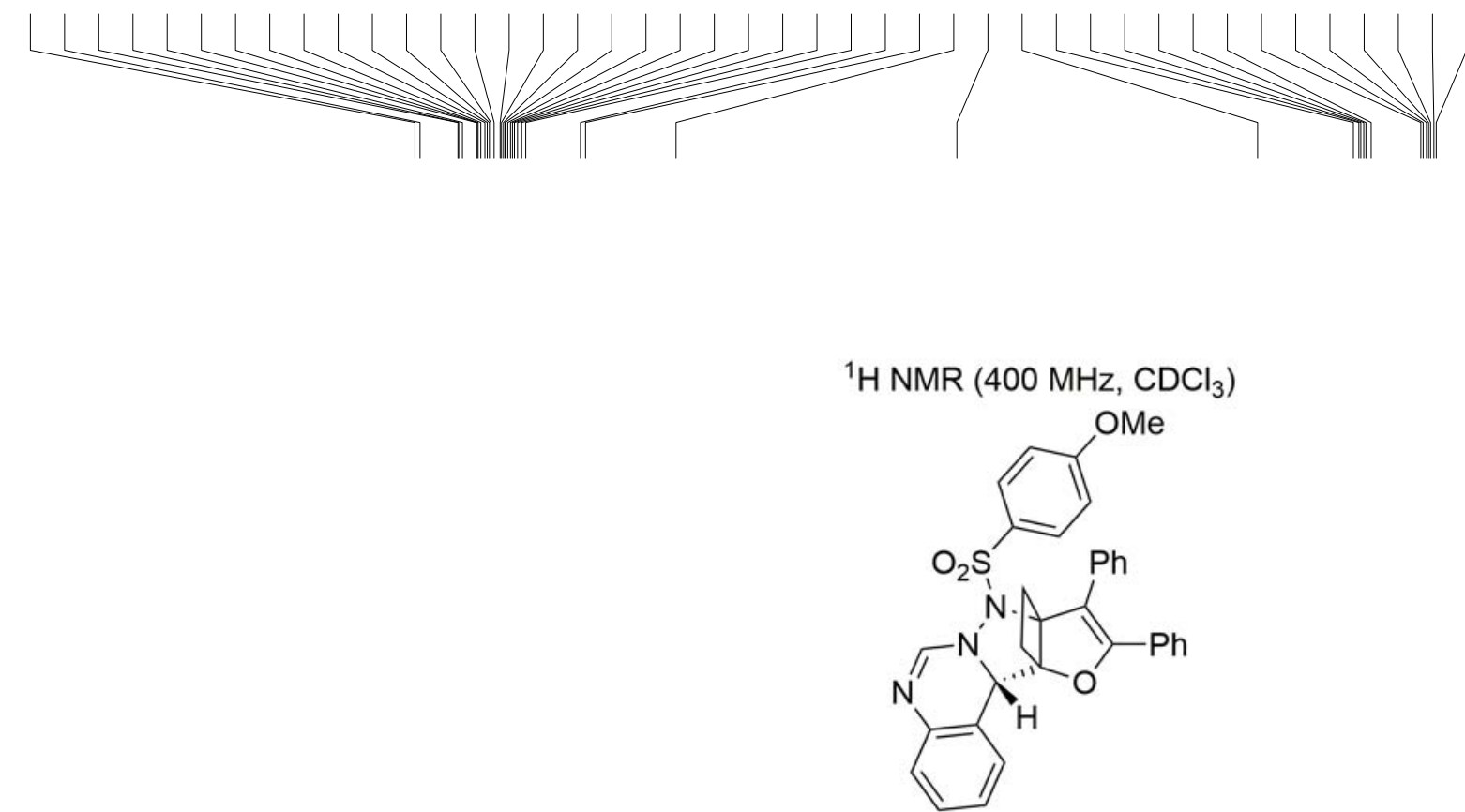

(5I) 


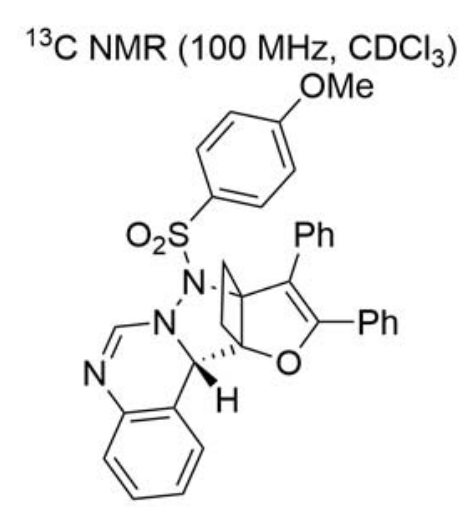

(51)

$\frac{1}{20}$




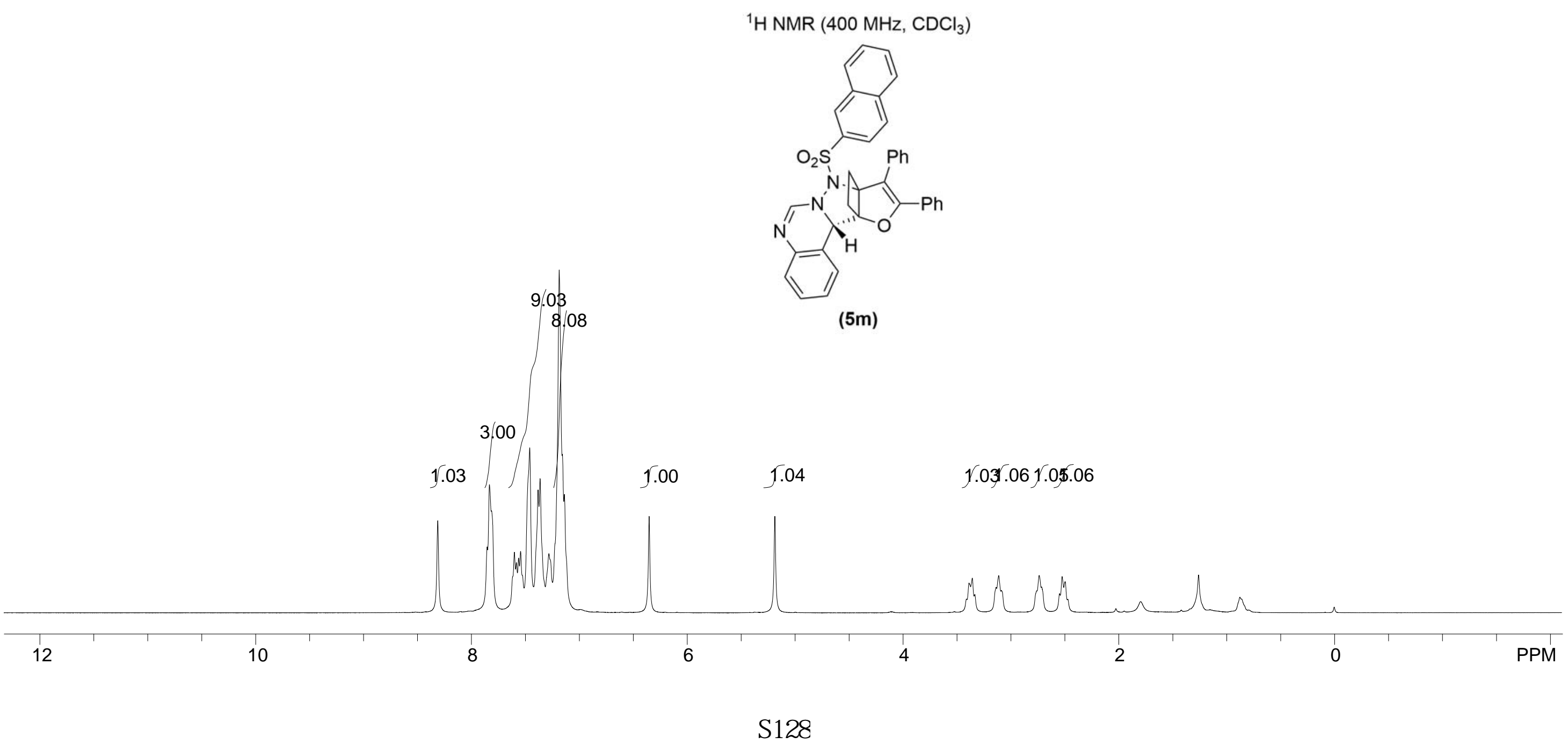




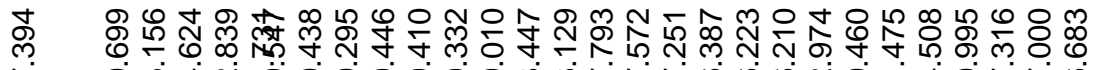

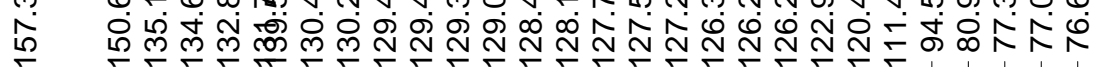

\section{${ }^{13} \mathrm{C} \mathrm{NMR}\left(100 \mathrm{MHz}, \mathrm{CDCl}_{3}\right)$}

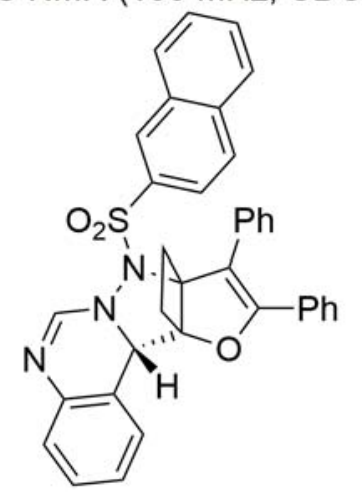

$(5 \mathrm{~m})$ 


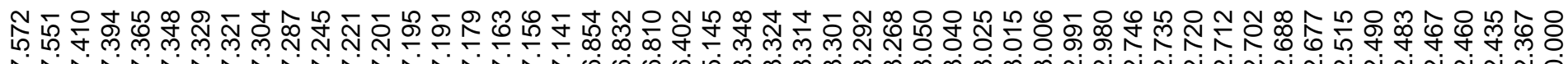

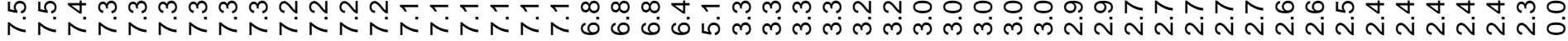
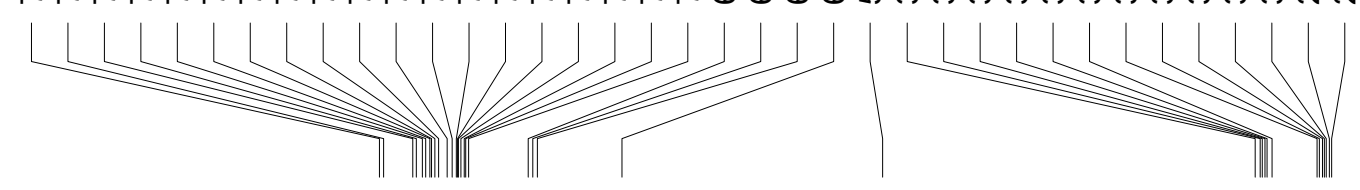

${ }^{1} \mathrm{H} \mathrm{NMR}\left(400 \mathrm{MHz}, \mathrm{CDCl}_{3}\right)$

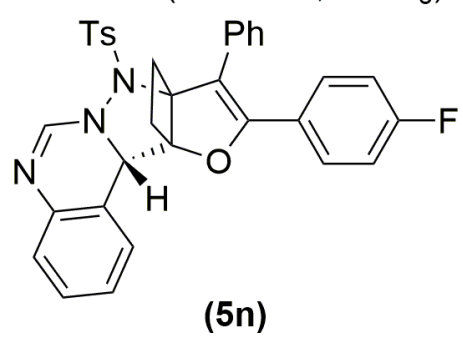

7.06

6.04 


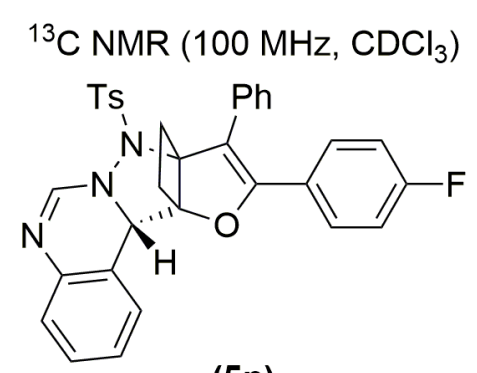

(5n) 


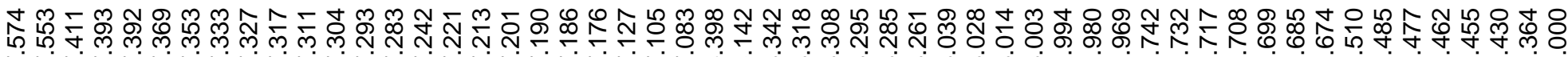

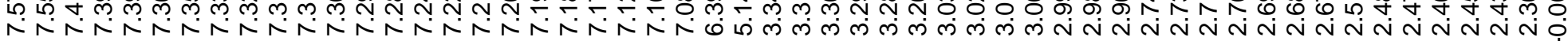
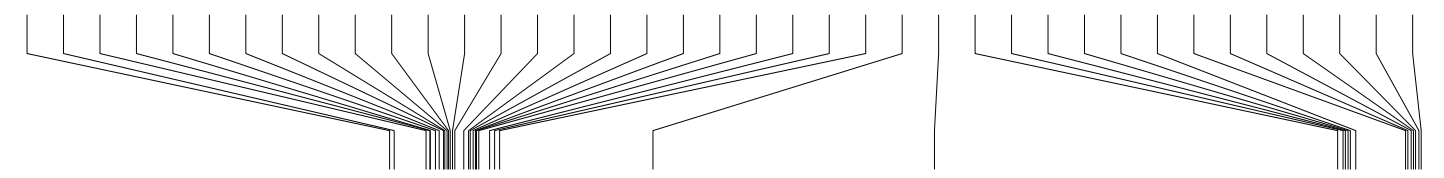

${ }^{1} \mathrm{H} \mathrm{NMR}\left(400 \mathrm{MHz}, \mathrm{CDCl}_{3}\right)$

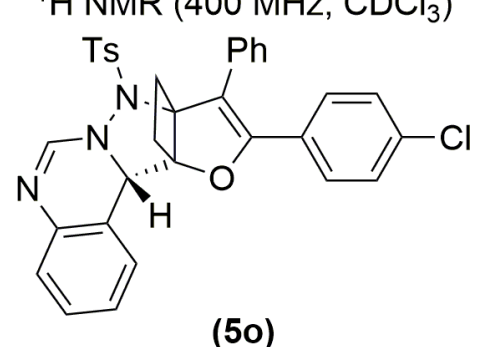

(50)

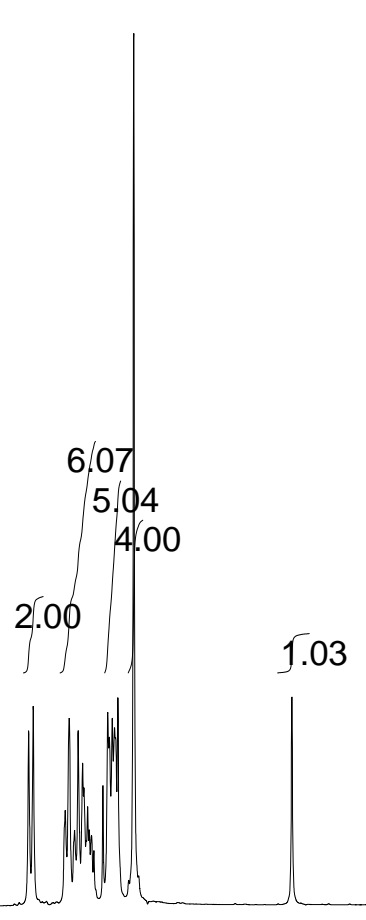

1.03

1.04

Wh 
${ }^{13} \mathrm{C}$ NMR $\left(100 \mathrm{MHz}, \mathrm{CDCl}_{3}\right)$

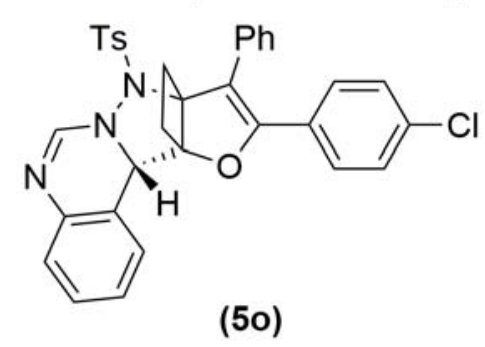




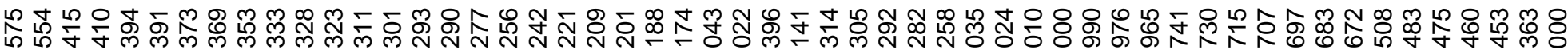

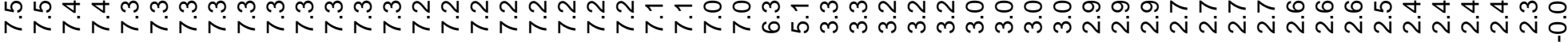
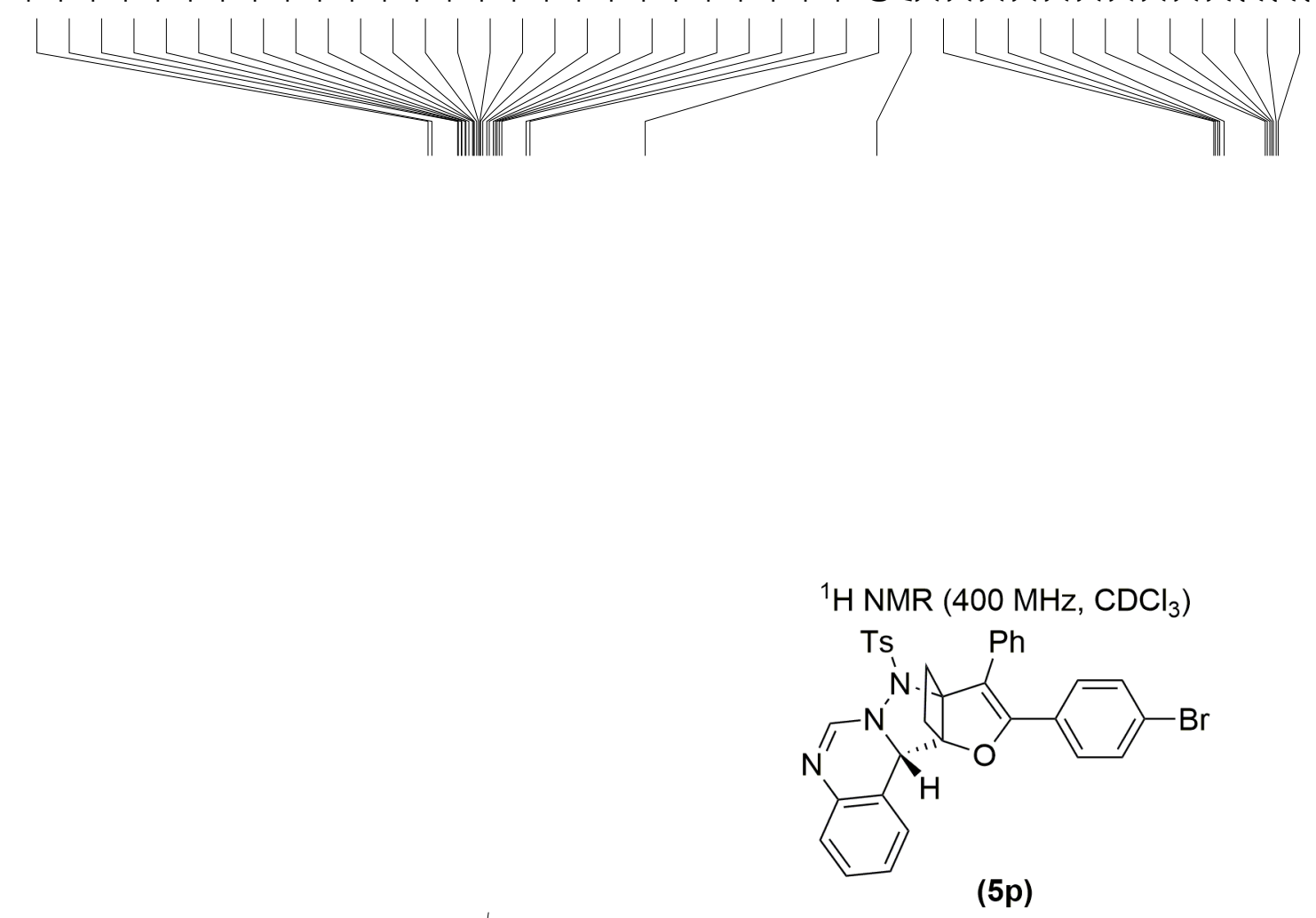

8.02

(5p)
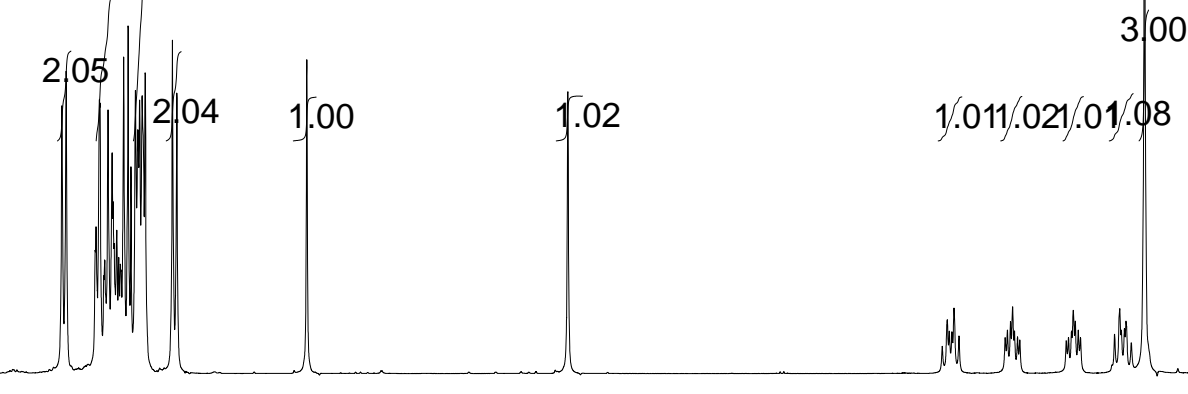


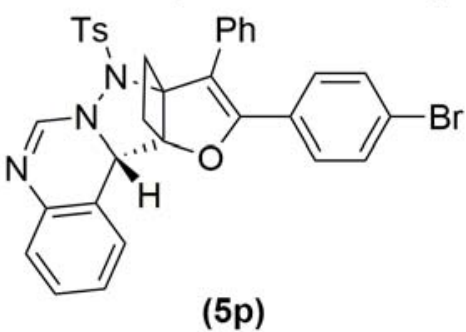




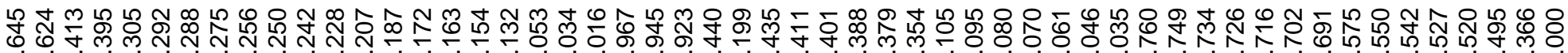

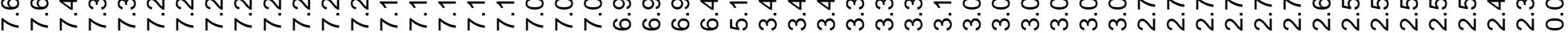
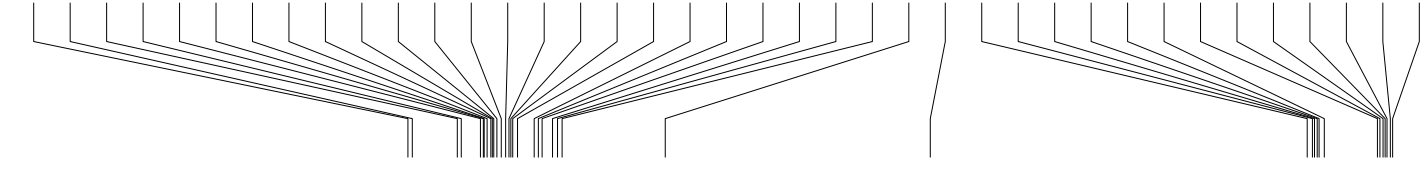

${ }^{1} \mathrm{H} \mathrm{NMR}\left(400 \mathrm{MHz}, \mathrm{CDCl}_{3}\right)$

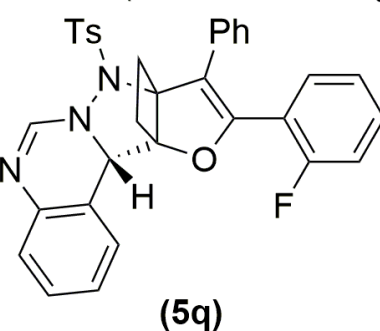


${ }^{13} \mathrm{C} \mathrm{NMR}\left(100 \mathrm{MHz}, \mathrm{CDCl}_{3}\right)$

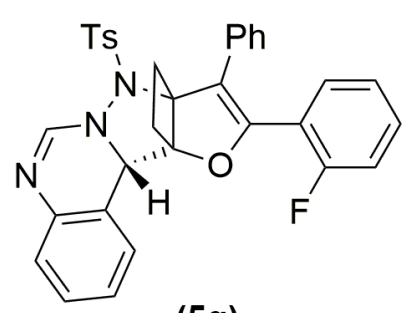

(5q) 
12.00

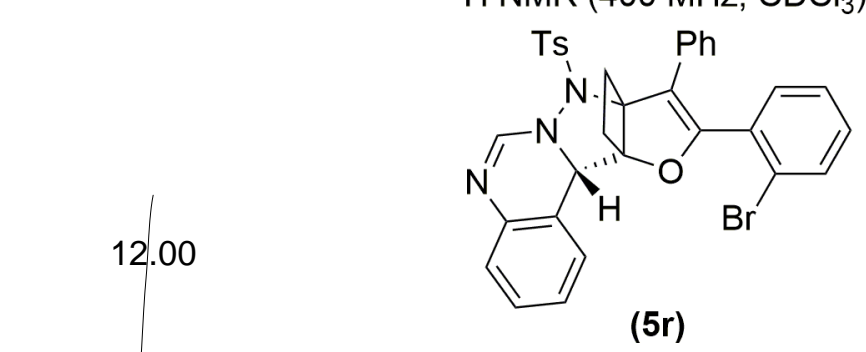

12

10

8

1.03

1.00

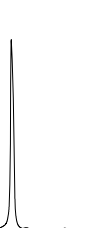

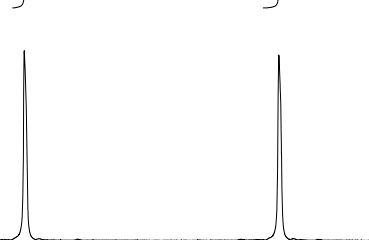

1.01

6

4

2

0

PPM 
${ }^{13} \mathrm{C} \mathrm{NMR}\left(100 \mathrm{MHz}, \mathrm{CDCl}_{3}\right)$

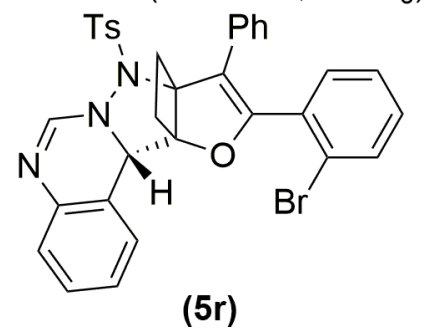


${ }^{1} \mathrm{H}$ NMR (400 MHz, $\mathrm{CDCl}_{3}$ )

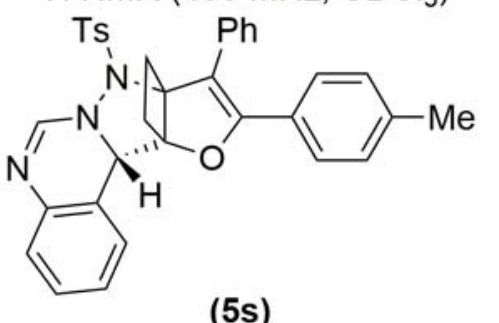

(5s) 


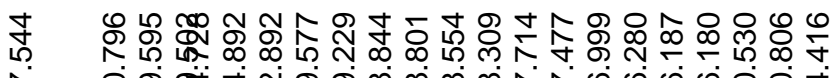

点

${ }^{13} \mathrm{C}$ NMR $\left(100 \mathrm{MHz}, \mathrm{CDCl}_{3}\right)$

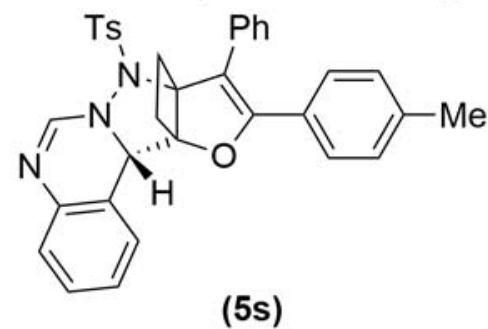

(5s) 


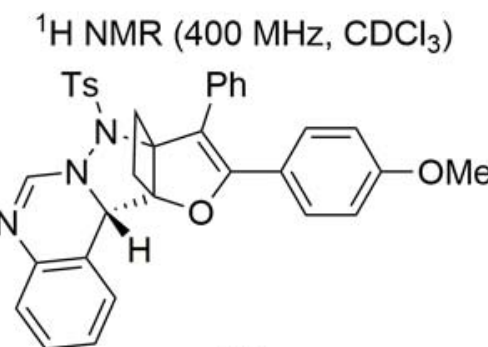

(5t)

1.01 


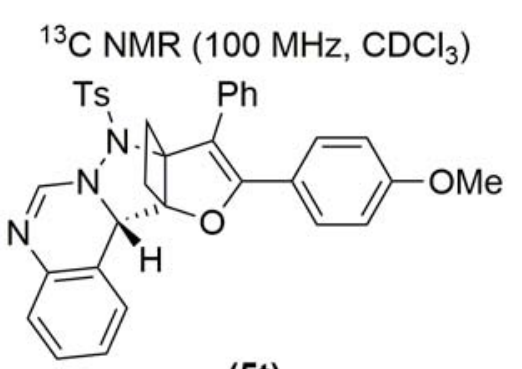

(5t) 


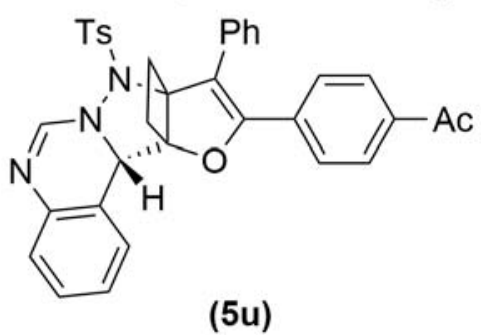




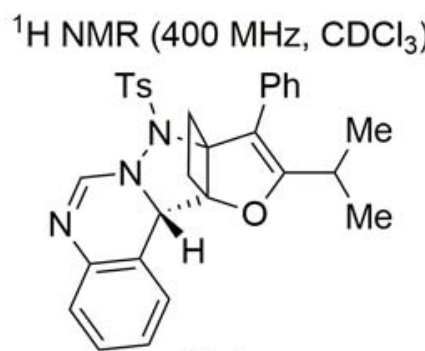

$(5 v)$

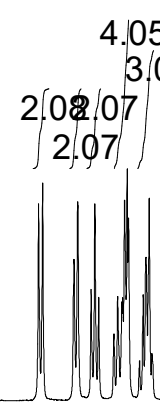

.05

2.08 .07

.07

1.04
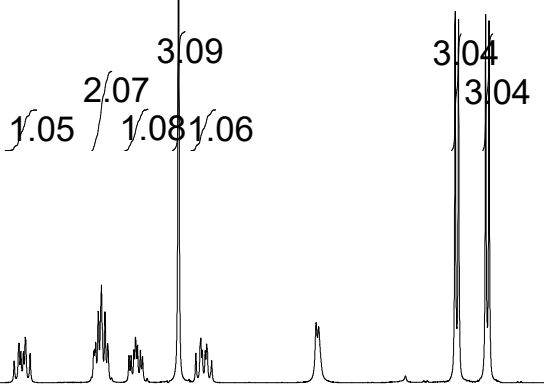
${ }^{13} \mathrm{C}$ NMR $\left(100 \mathrm{MHz}, \mathrm{CDCl}_{3}\right.$ )

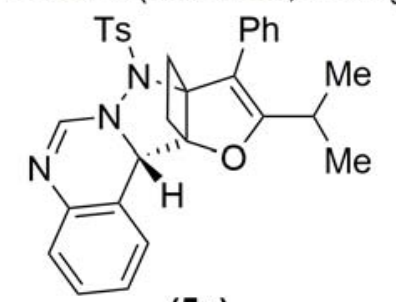

(5v) 


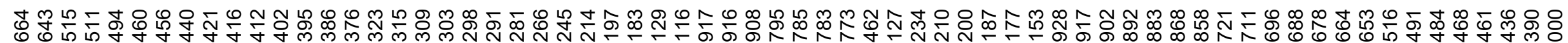

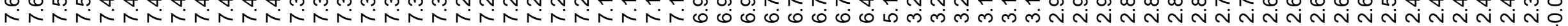

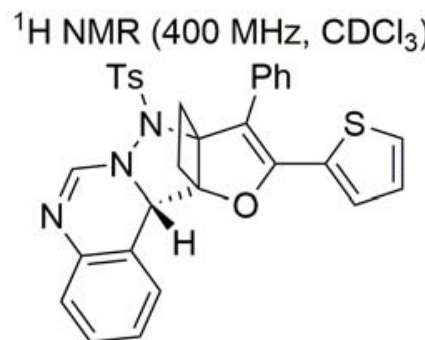

$(5 w)$
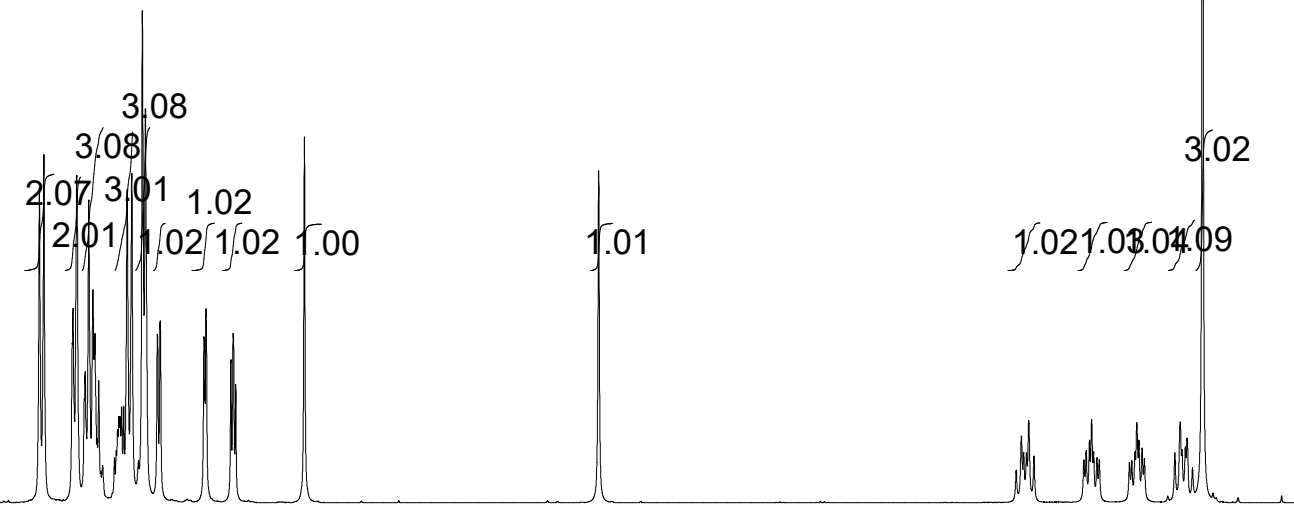
${ }^{13} \mathrm{C} \mathrm{NMR}\left(100 \mathrm{MHz}, \mathrm{CDCl}_{3}\right)$

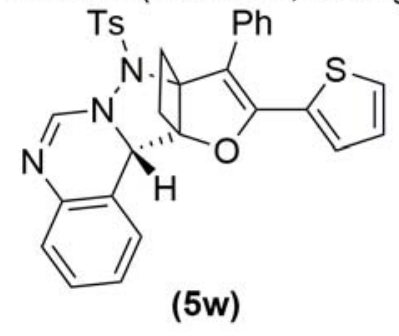



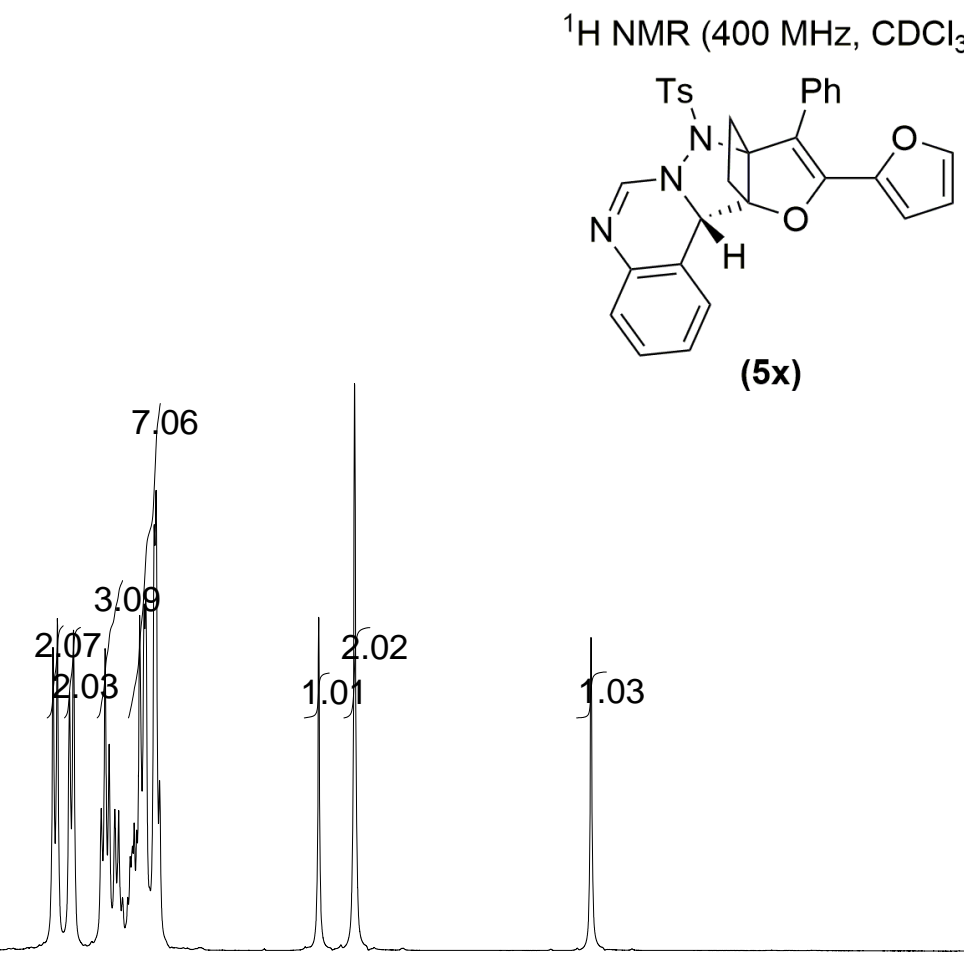

$(5 x)$
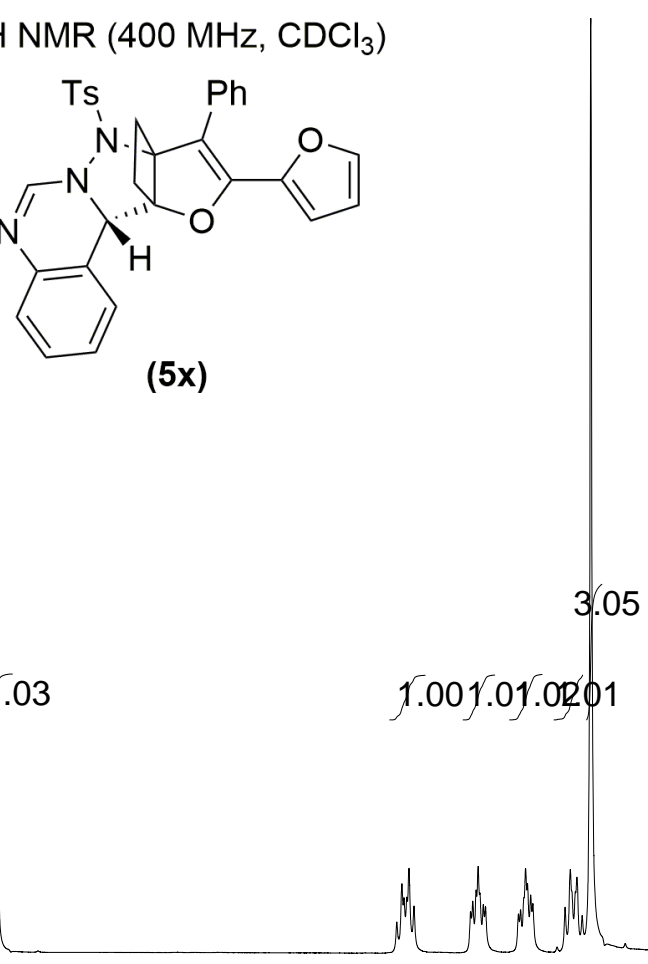


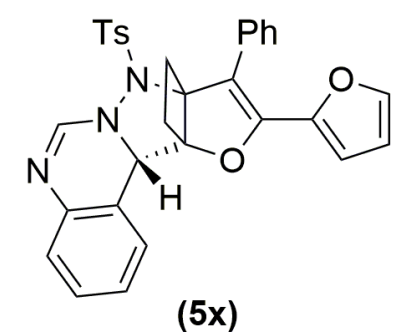

$(5 x)$ 


\section{${ }^{1} \mathrm{H}$ NMR $\left(400 \mathrm{MHz}, \mathrm{CDCl}_{3}\right)$}

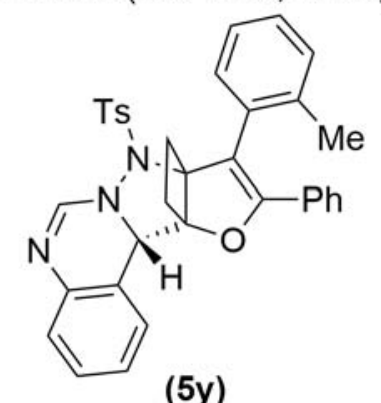

(5y)

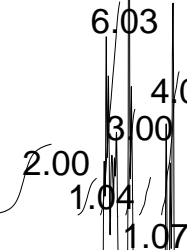

4. 05 


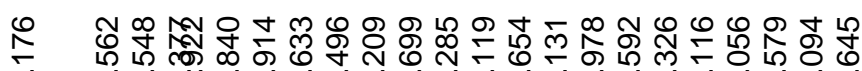

它

${ }^{13} \mathrm{C} \mathrm{NMR}\left(100 \mathrm{MHz}, \mathrm{CDCl}_{3}\right)$

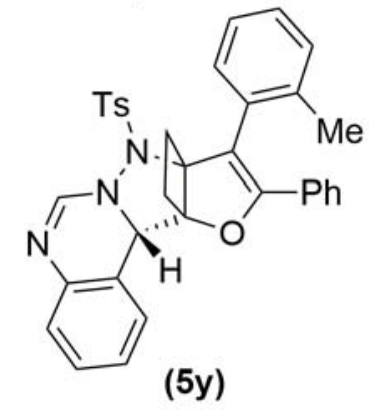

(5y)

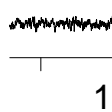




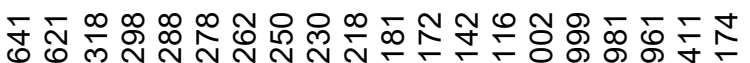

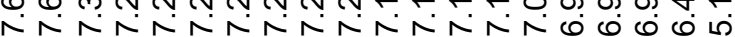

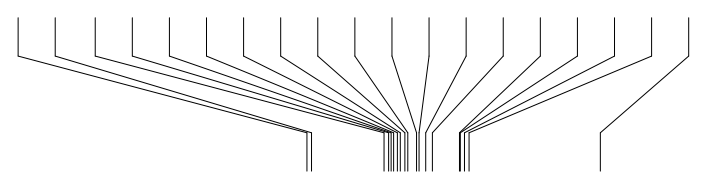

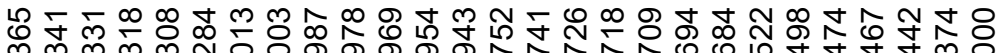

అ

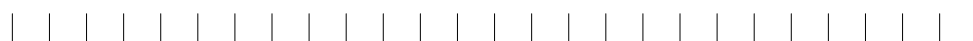

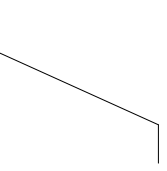

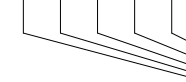

M $1 \|$

${ }^{1} \mathrm{H}$ NMR $\left(400 \mathrm{MHz}, \mathrm{CDCl}_{3}\right.$ )

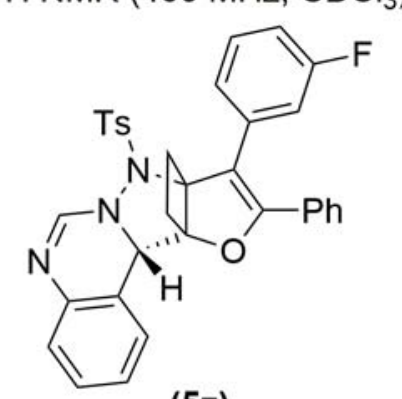

(5z)

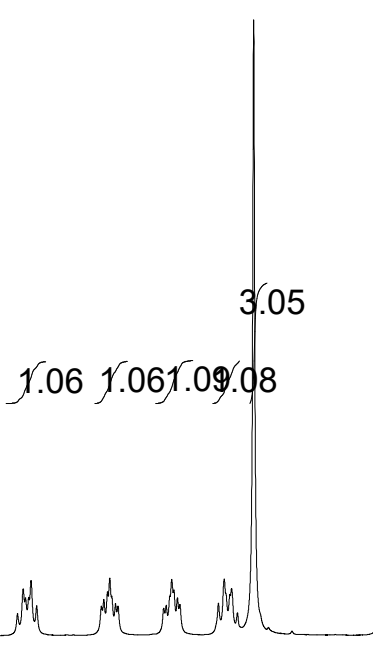




\section{${ }^{13} \mathrm{C}$ NMR $\left(100 \mathrm{MHz}, \mathrm{CDCl}_{3}\right)$}

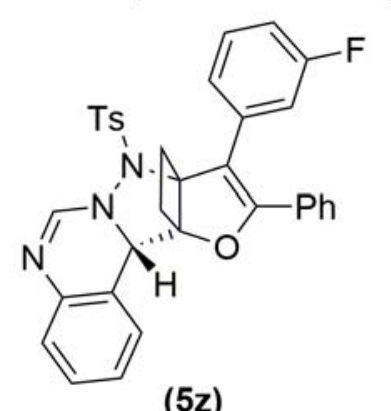

$(5 z)$ 


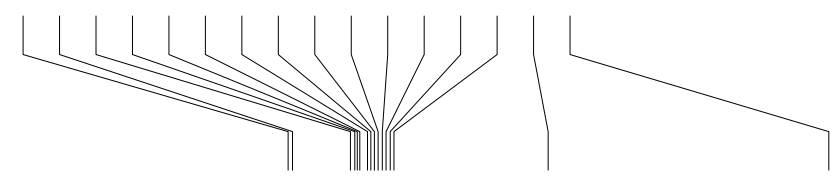

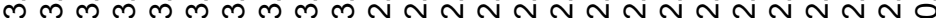

${ }^{1} \mathrm{H} \mathrm{NMR}\left(400 \mathrm{MHz}, \mathrm{CDCl}_{3}\right)$

12.00

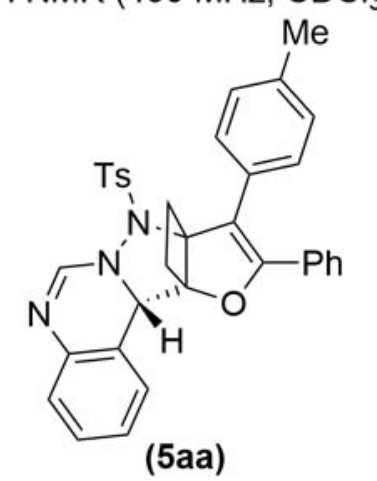




\section{${ }^{13} \mathrm{C} \mathrm{NMR}\left(100 \mathrm{MHz}, \mathrm{CDCl}_{3}\right)$}

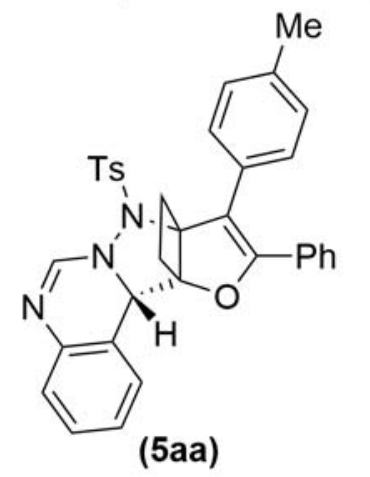

(5aa) 


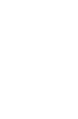

3.06
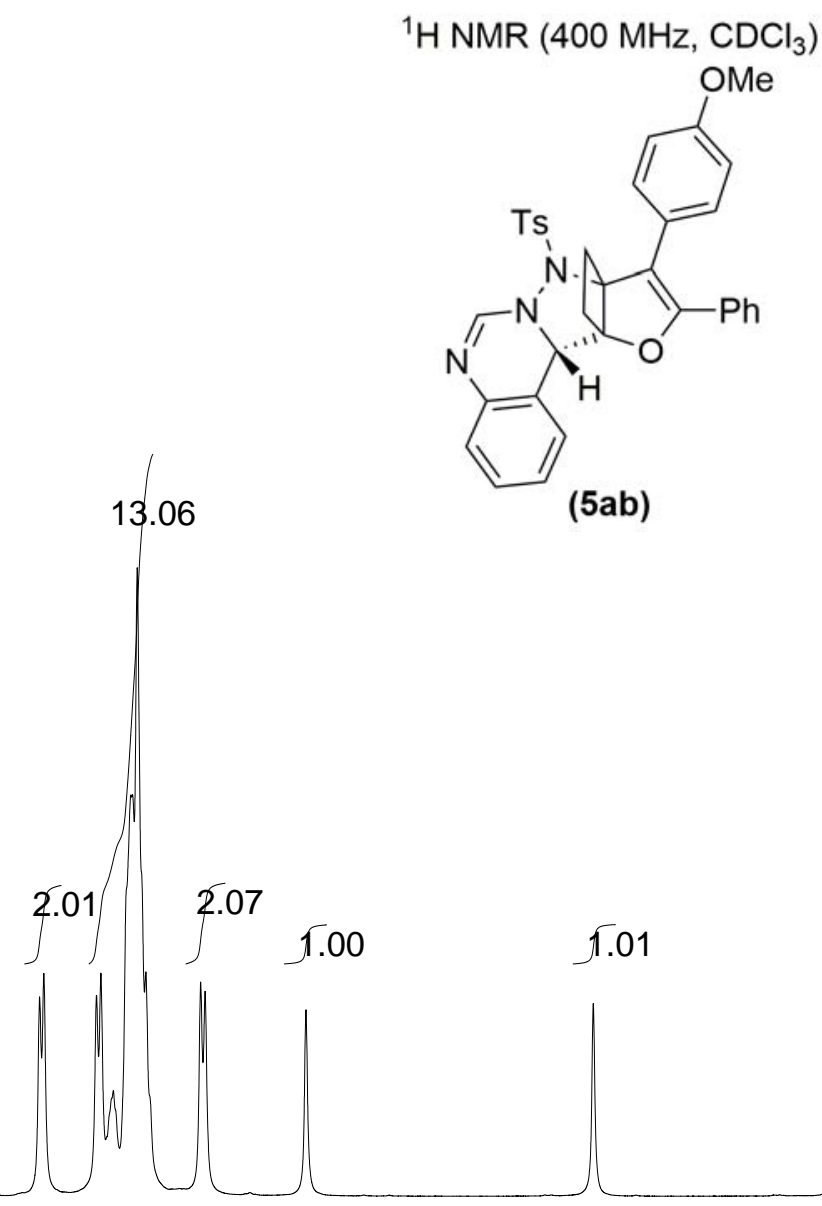


\section{${ }^{13} \mathrm{C}$ NMR $\left(100 \mathrm{MHz}, \mathrm{CDCl}_{3}\right)$}
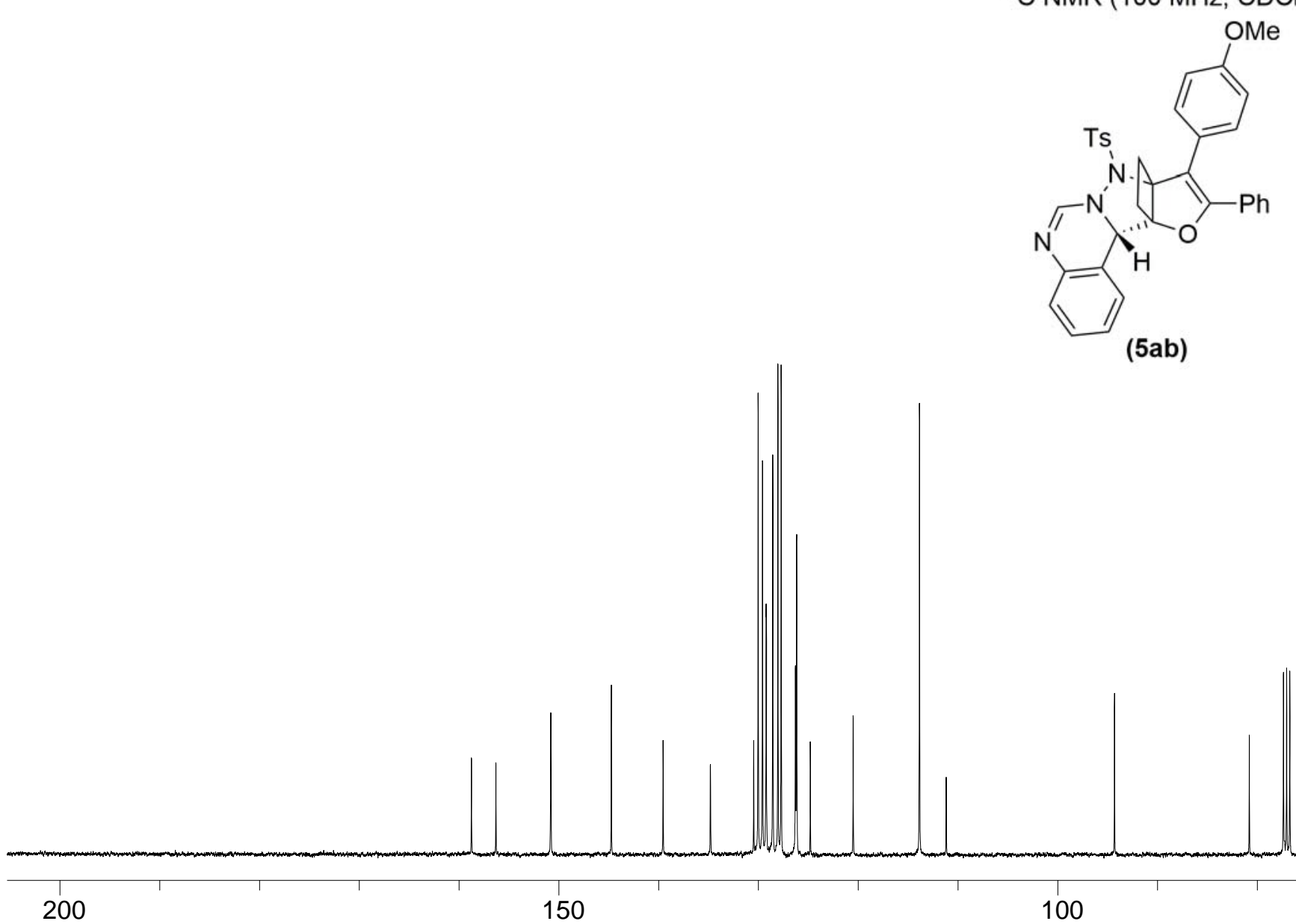


\section{${ }^{13} \mathrm{C}$ NMR $\left(100 \mathrm{MHz}, \mathrm{CDCl}_{3}\right)$}

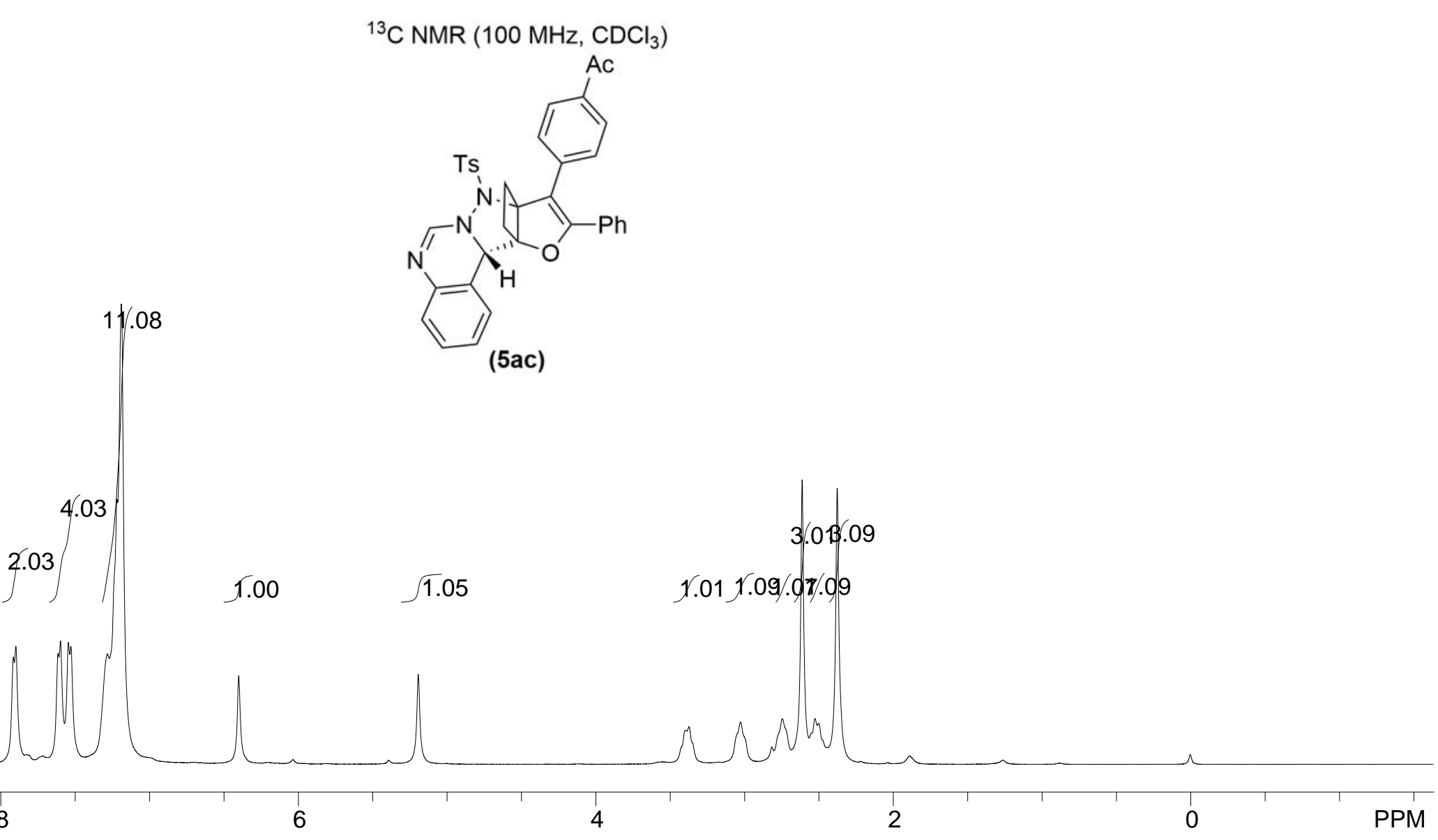




\section{${ }^{1} \mathrm{H} \mathrm{NMR}\left(400 \mathrm{MHz}, \mathrm{CDCl}_{3}\right)$}

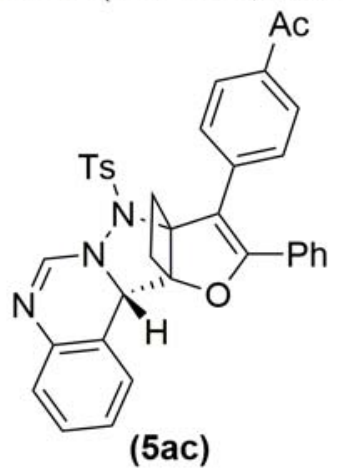


${ }^{1} \mathrm{H} \mathrm{NMR}\left(400 \mathrm{MHz}, \mathrm{CDCl}_{3}\right)$

11.04

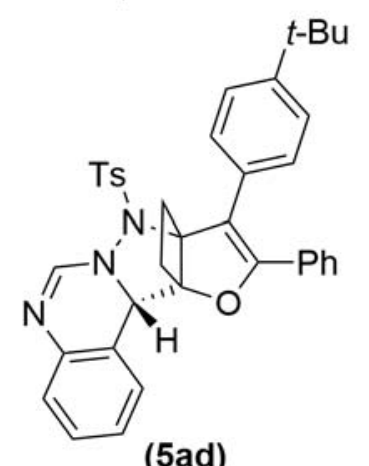

(5ad)

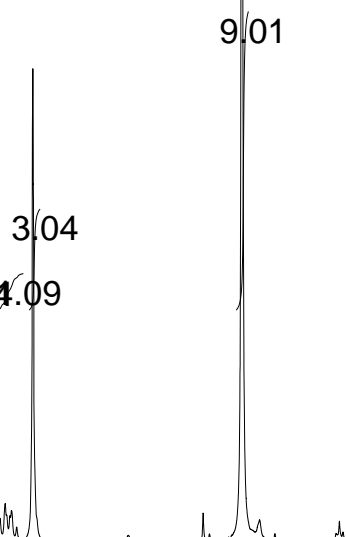

\section{S162}




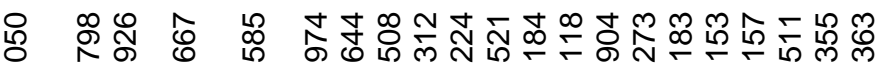

官

${ }^{13} \mathrm{C}$ NMR $\left(100 \mathrm{MHz}, \mathrm{CDCl}_{3}\right)$

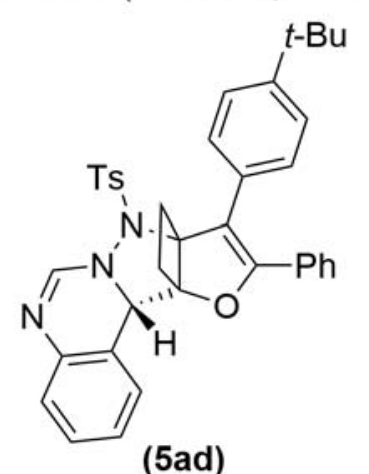


${ }^{1} \mathrm{H}$ NMR (400 MHz, $\left.\mathrm{CDCl}_{3}\right)$
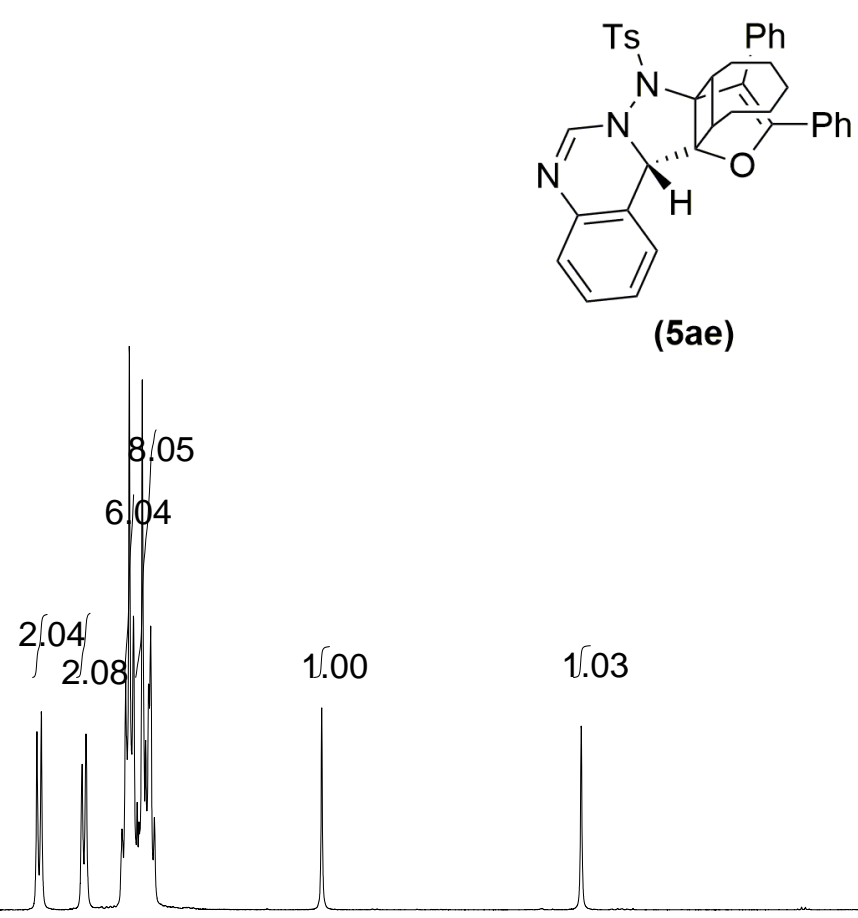

(5ae) 


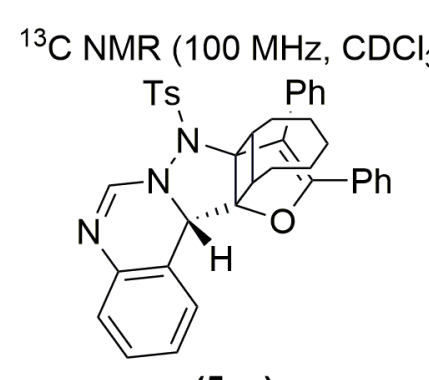

(5ae) 


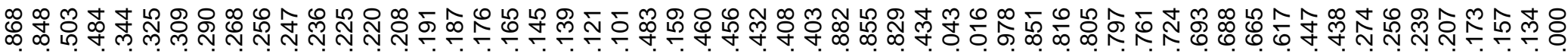

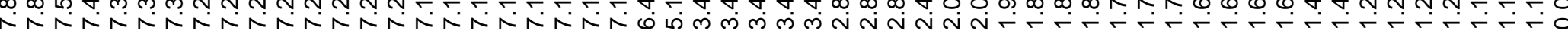

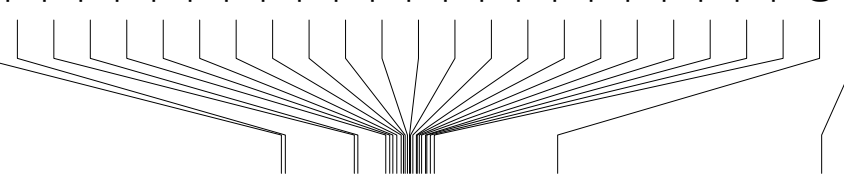

${ }^{1} \mathrm{H} \mathrm{NMR}\left(400 \mathrm{MHz}, \mathrm{CDCl}_{3}\right)$

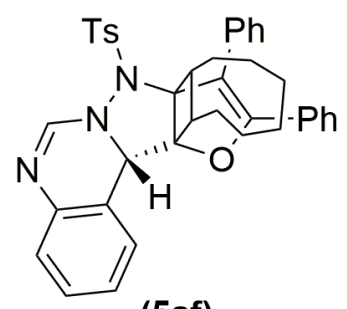

(5af)
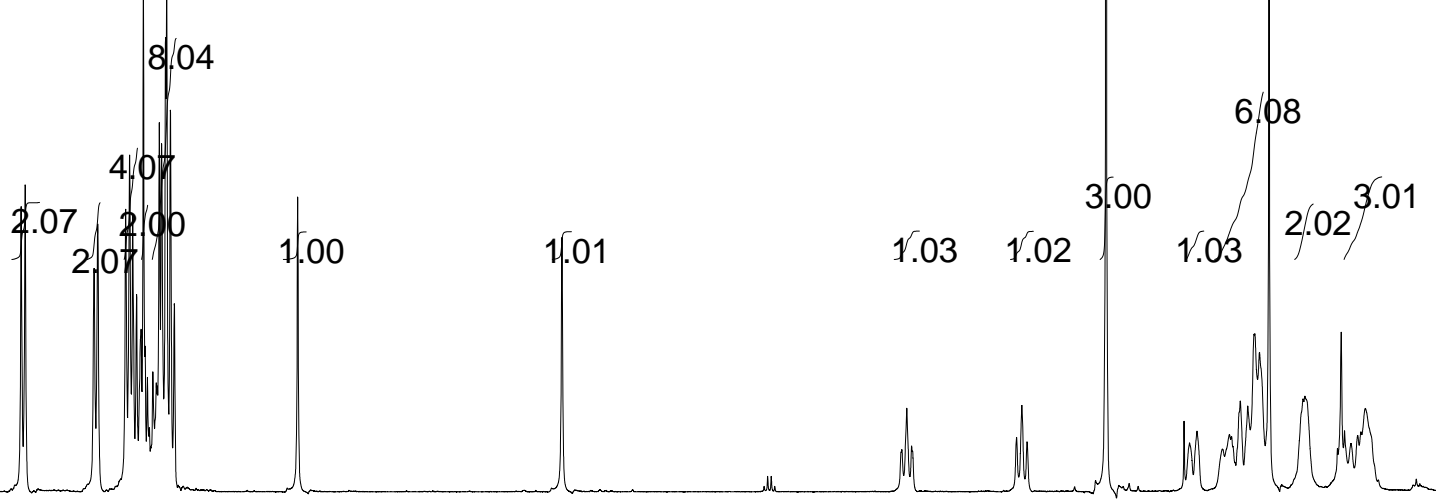


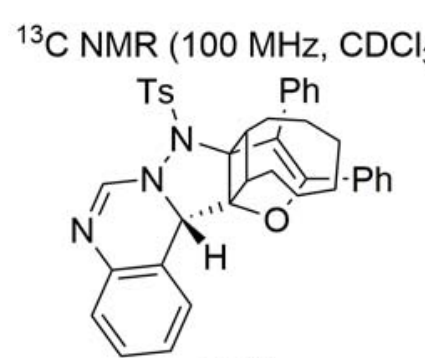

(5af) 


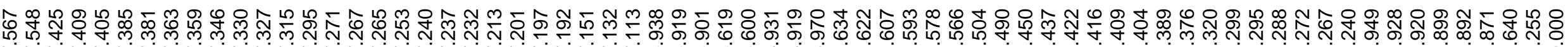

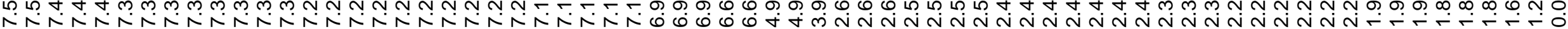

${ }^{1} \mathrm{H} \mathrm{NMR}\left(400 \mathrm{MHz}, \mathrm{CDCl}_{3}\right)$

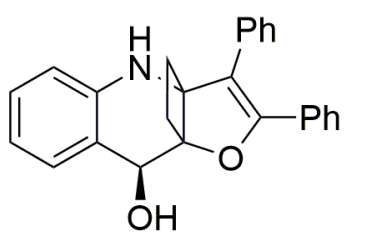

(6) 
${ }^{13} \mathrm{C}$ NMR $\left(100 \mathrm{MHz}, \mathrm{CDCl}_{3}\right)$

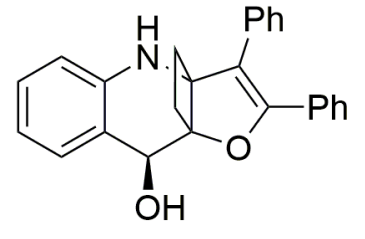

(6)

$$
20
$$




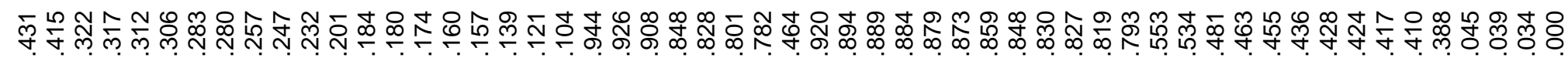

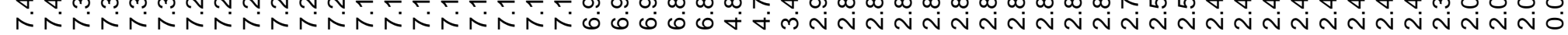
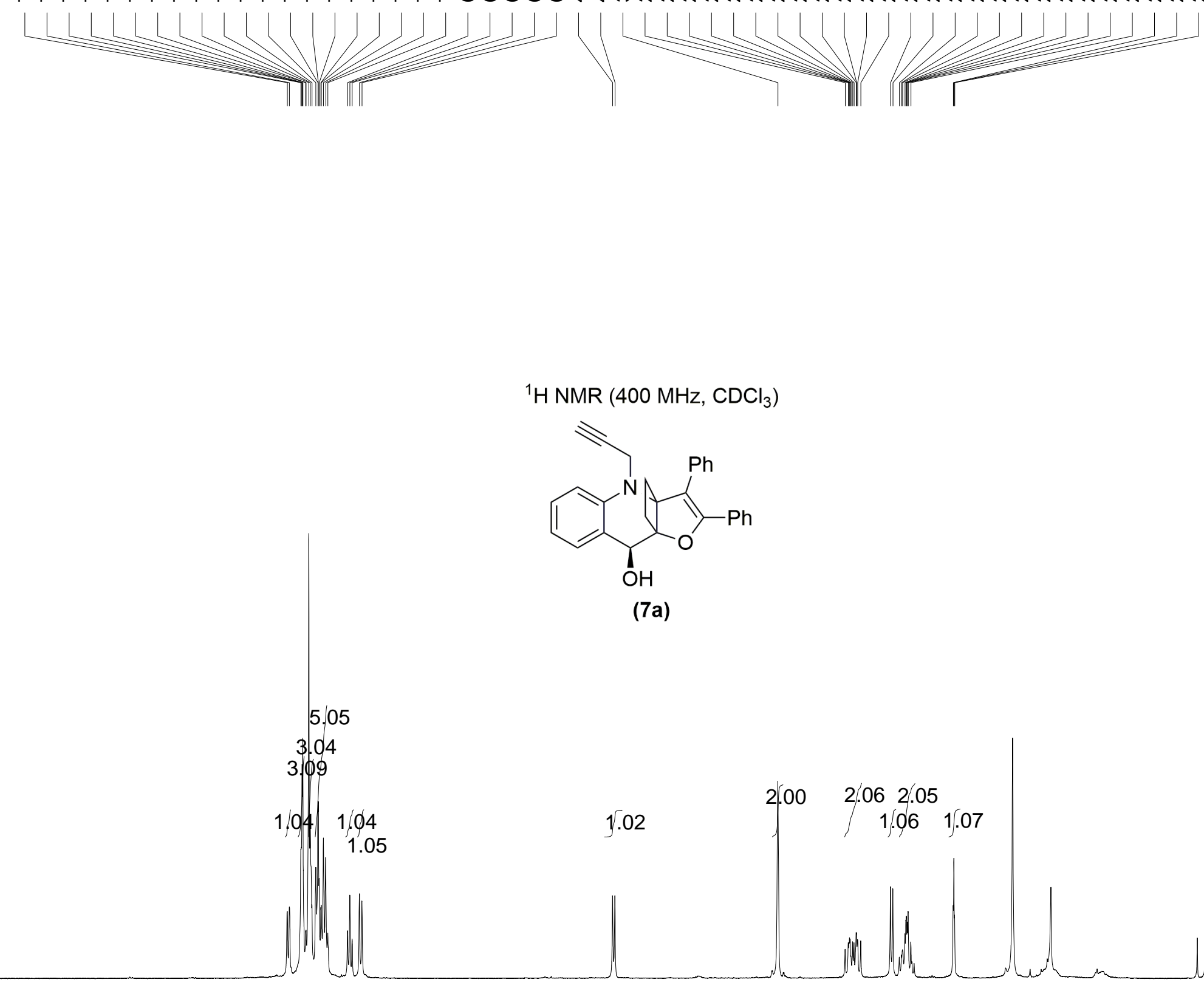
${ }^{13} \mathrm{C}$ NMR $\left(100 \mathrm{MHz}, \mathrm{CDCl}_{3}\right)$

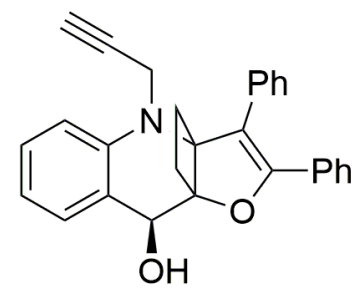

(7a)

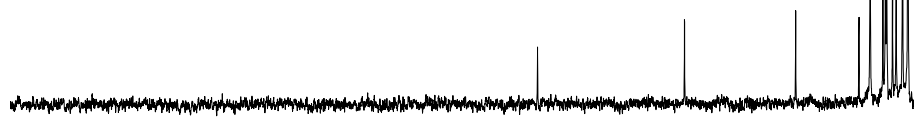


${ }^{1} \mathrm{H}$ NMR $\left(400 \mathrm{MHz}, \mathrm{CDCl}_{3}\right)$

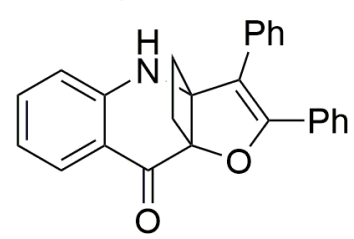

(7b)
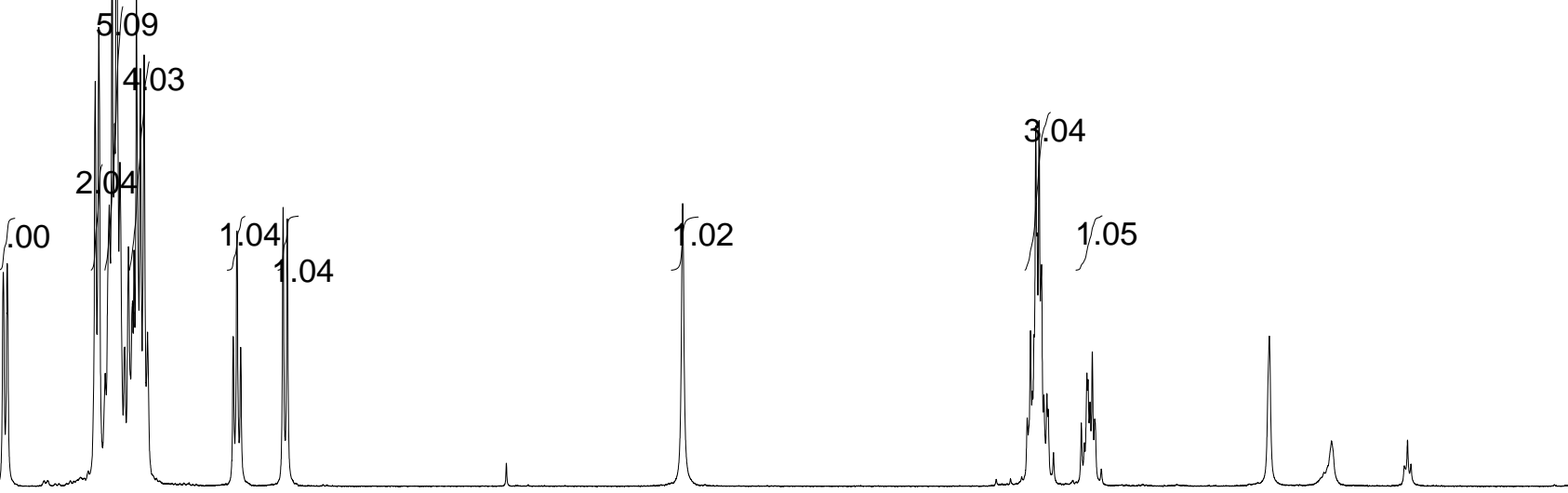
${ }^{13} \mathrm{C} \mathrm{NMR}\left(100 \mathrm{MHz}, \mathrm{CDCl}_{3}\right)$
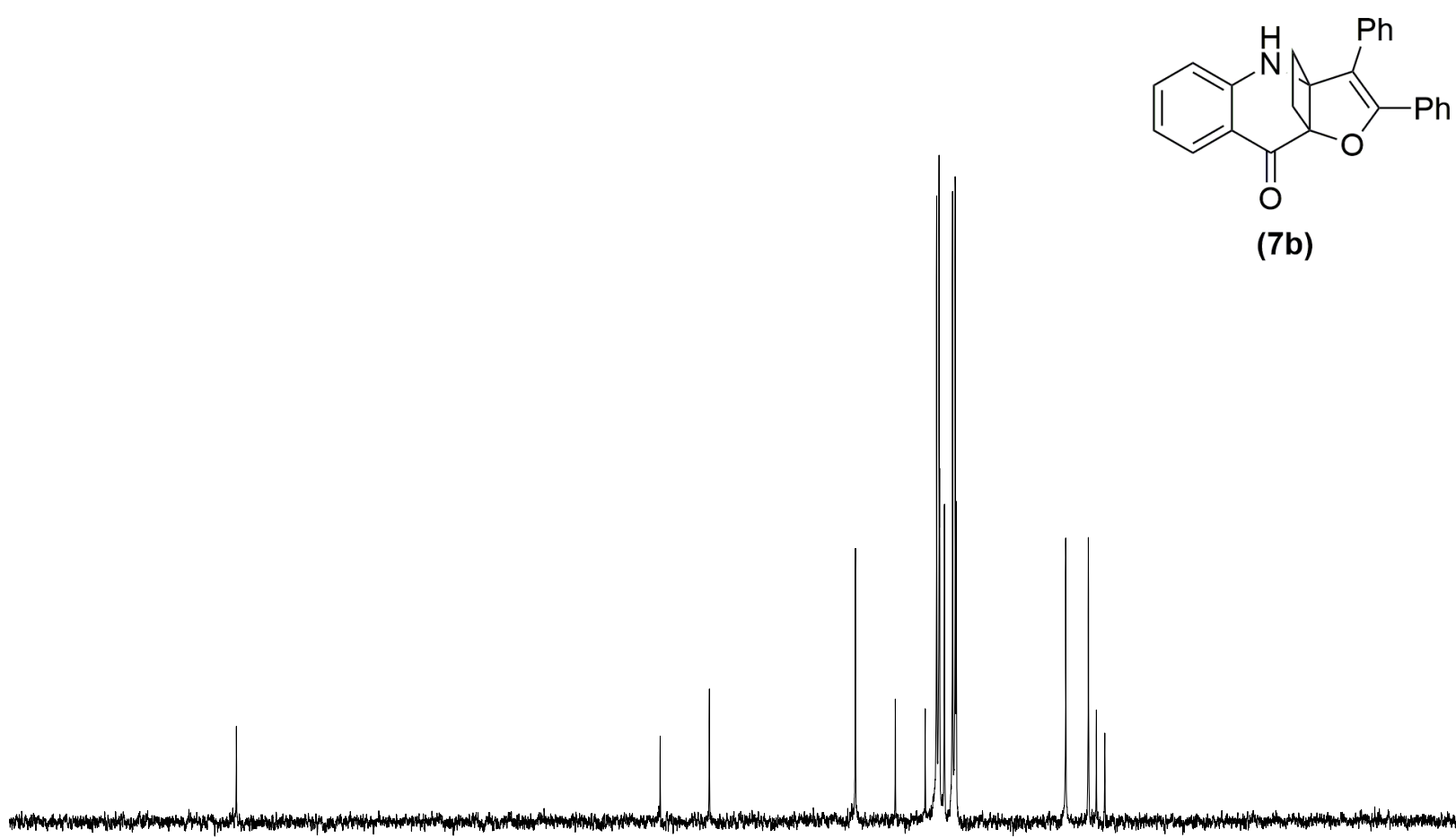

(7b) 


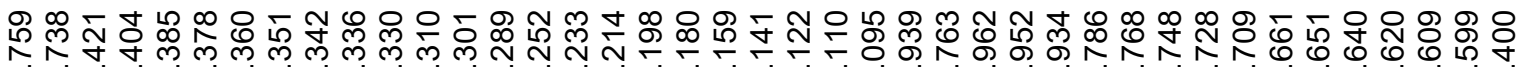

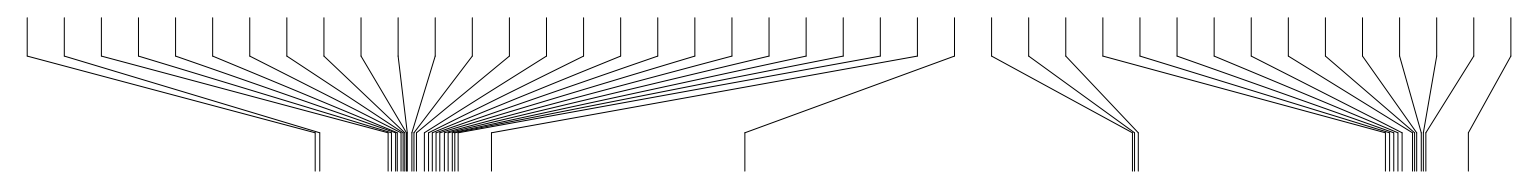

${ }^{1} \mathrm{H}$ NMR $\left(400 \mathrm{MHz}, \mathrm{CDCl}_{3}\right)$

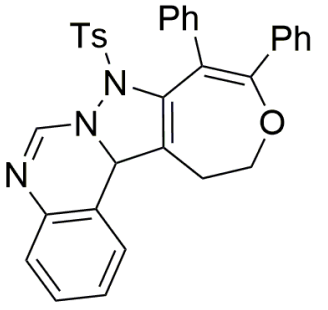

10.08

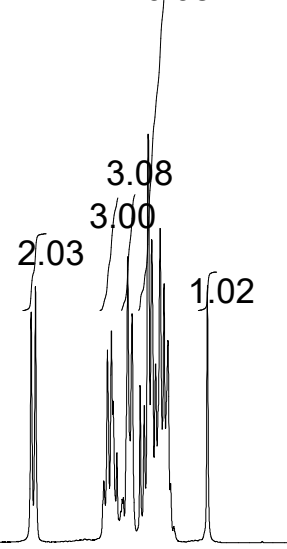

(8)

1.00 
${ }^{13} \mathrm{C}$ NMR $\left(100 \mathrm{MHz}, \mathrm{CDCl}_{3}\right.$ )

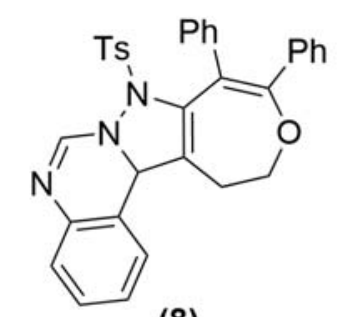

(8) 


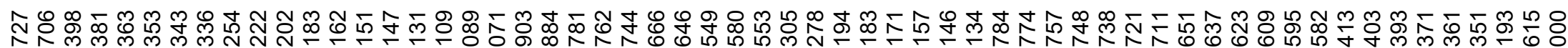

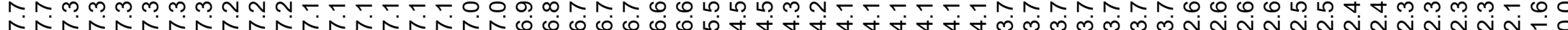
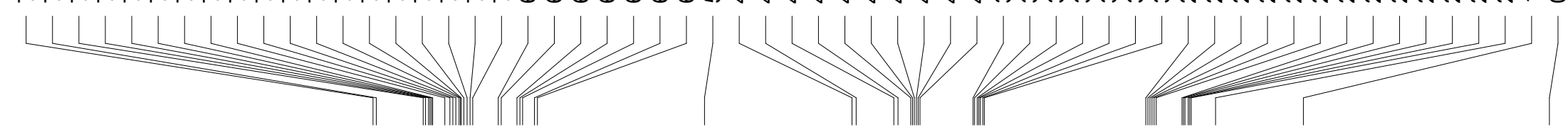

${ }^{1} \mathrm{H}$ NMR $\left(400 \mathrm{MHz}, \mathrm{CDCl}_{3}\right)$

10.06

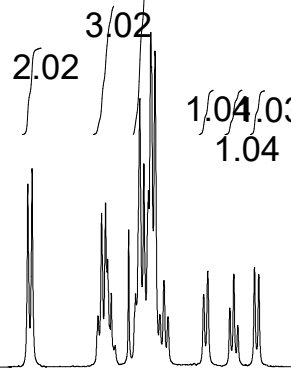

(9)

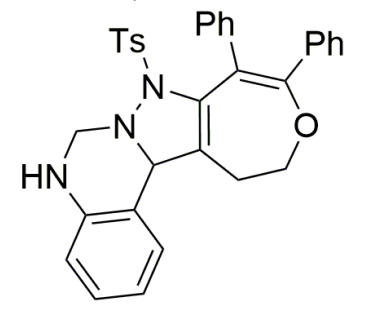


${ }^{13} \mathrm{C} \mathrm{NMR}\left(100 \mathrm{MHz}, \mathrm{CDCl}_{3}\right)$

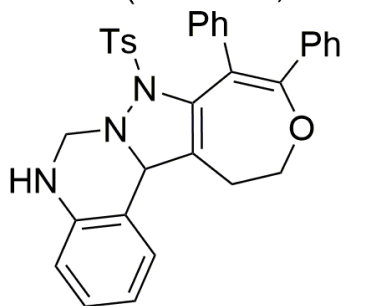

(9) 


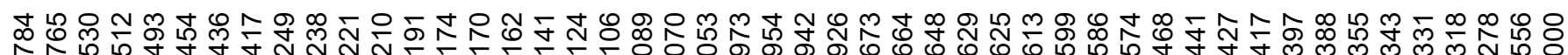

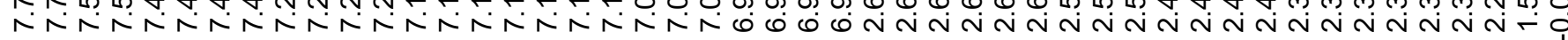
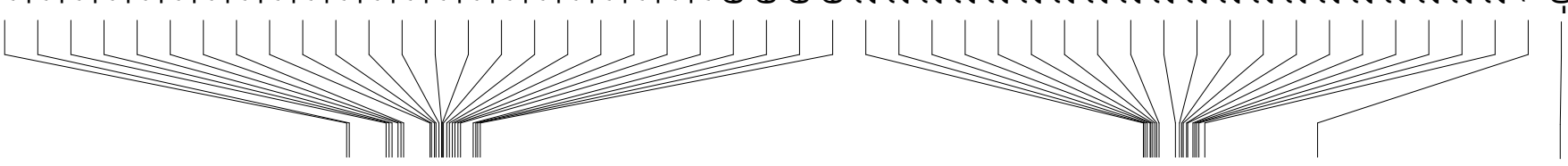

${ }^{1} \mathrm{H} \mathrm{NMR}\left(400 \mathrm{MHz}, \mathrm{CDCl}_{3}\right)$
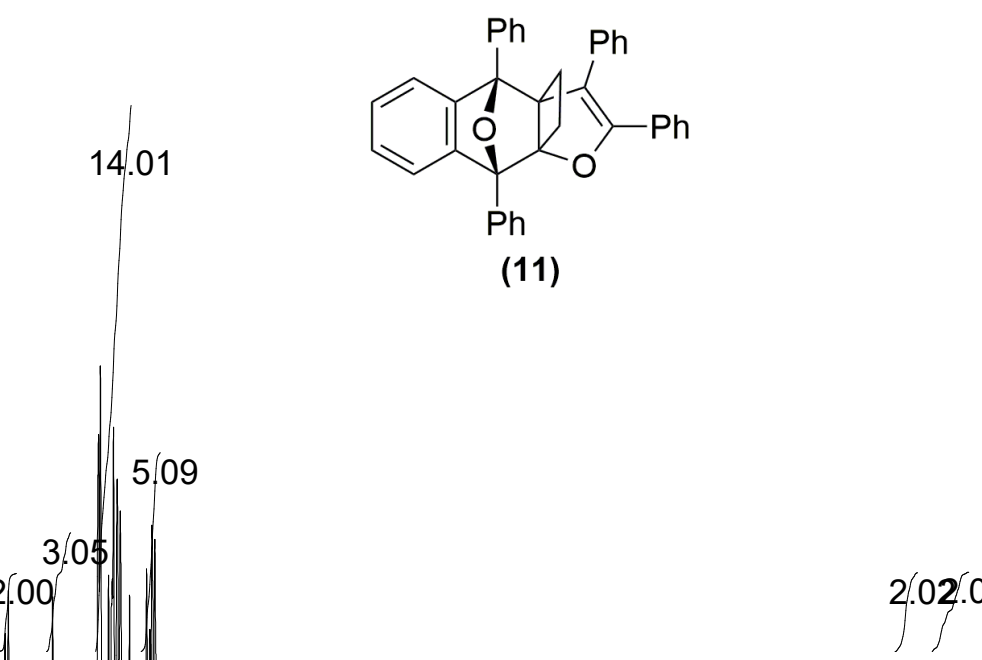
${ }^{13} \mathrm{C} \mathrm{NMR}\left(100 \mathrm{MHz}, \mathrm{CDCl}_{3}\right)$

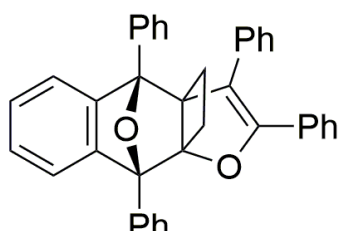

(11) 


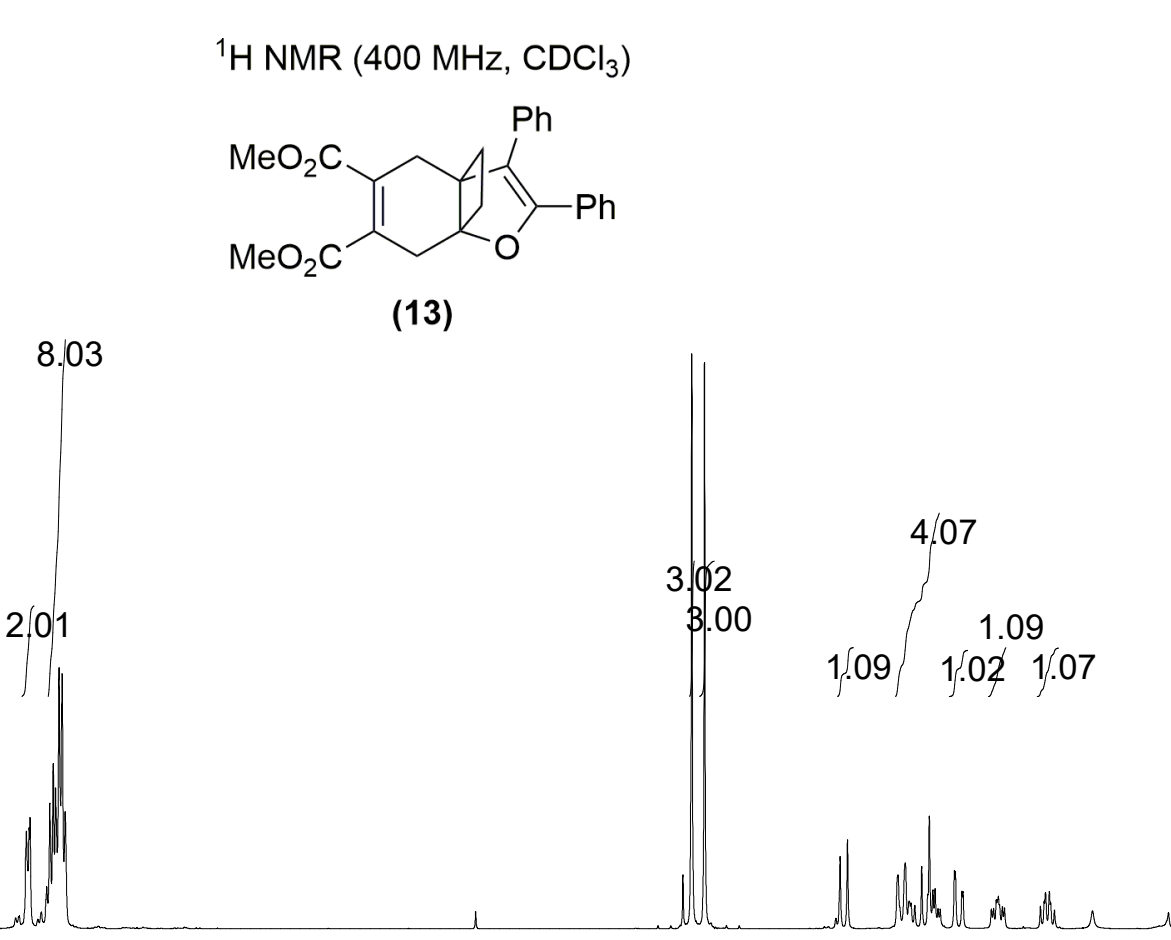




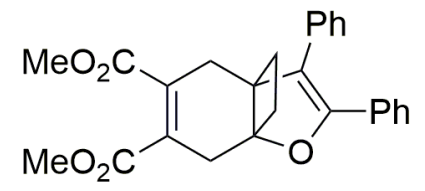

(13)

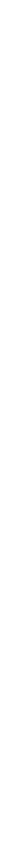




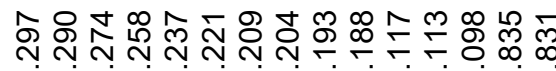

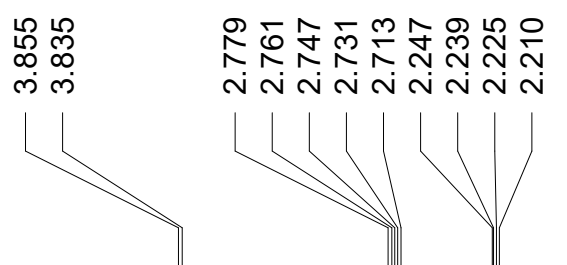

${ }^{1} \mathrm{H} \mathrm{NMR}\left(400 \mathrm{MHz}, \mathrm{CDCl}_{3}\right)$

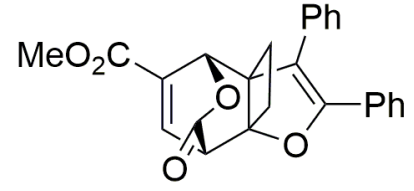

(15)

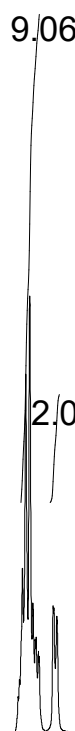


${ }^{13} \mathrm{C}$ NMR $\left(100 \mathrm{MHz}, \mathrm{CDCl}_{3}\right)$

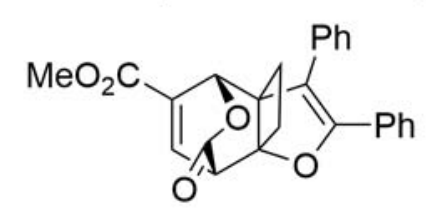

(15) 


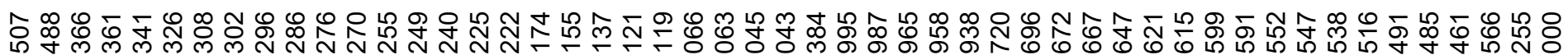

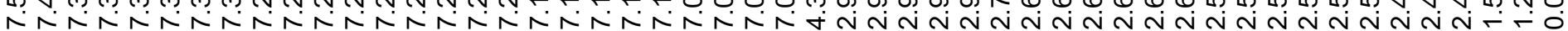

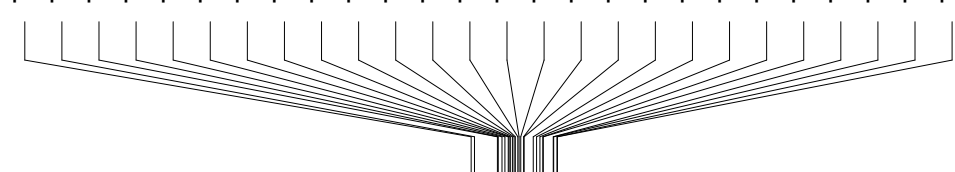

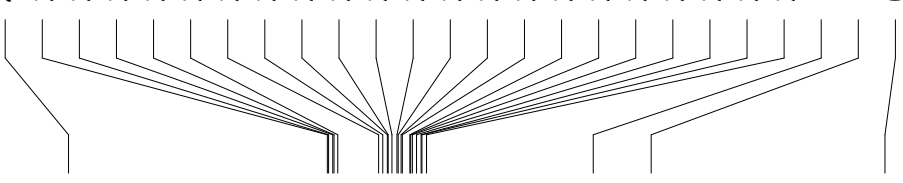

9.08

${ }^{1} \mathrm{H} \mathrm{NMR}\left(400 \mathrm{MHz}, \mathrm{CDCl}_{3}\right)$
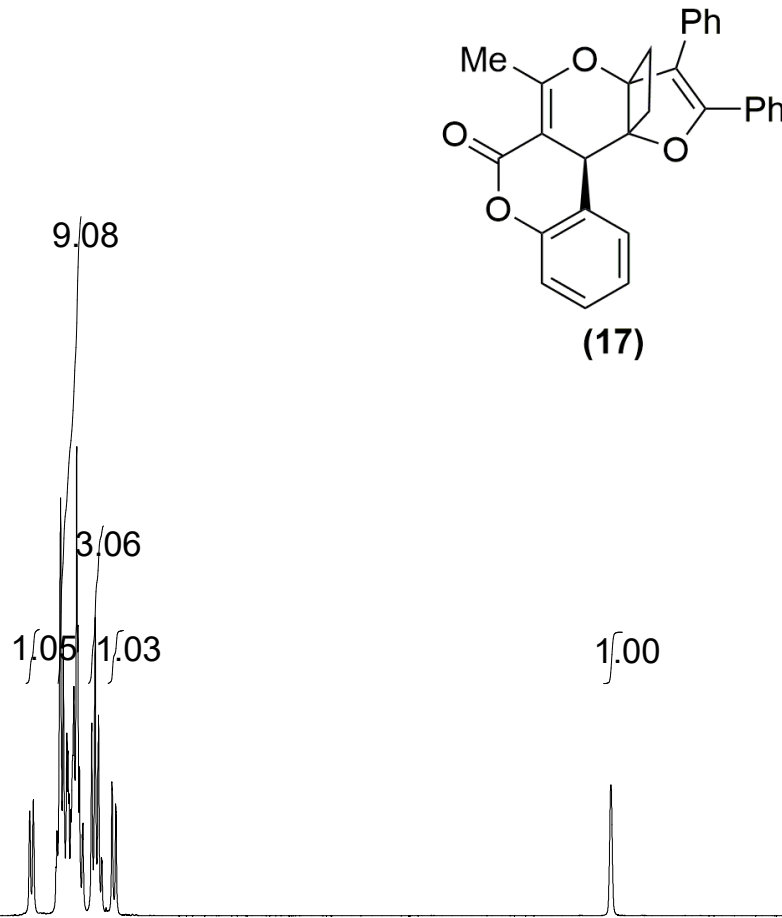

(17) 
${ }^{13} \mathrm{C}$ NMR $\left(100 \mathrm{MHz}, \mathrm{CDCl}_{3}\right)$

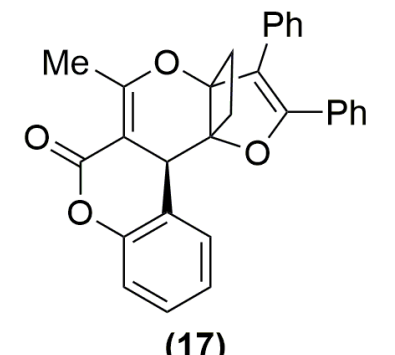

(17) 


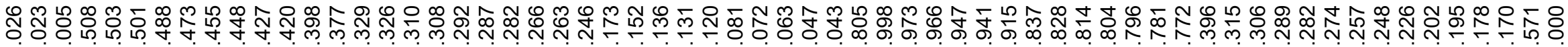
m

${ }^{1} \mathrm{H} \mathrm{NMR}\left(400 \mathrm{MHz}, \mathrm{CDCl}_{3}\right)$
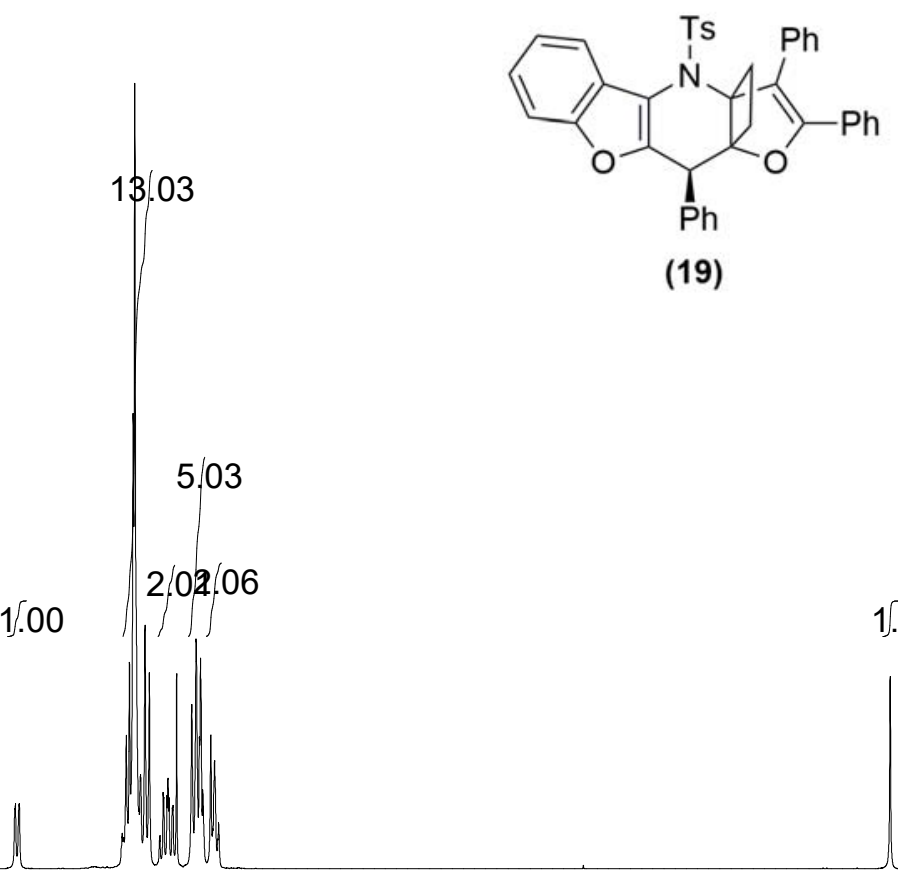

(19) 


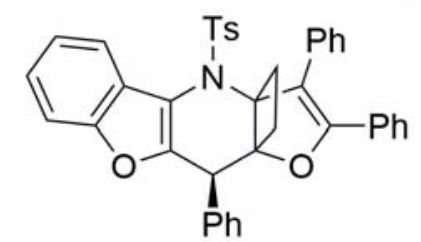

(19) 


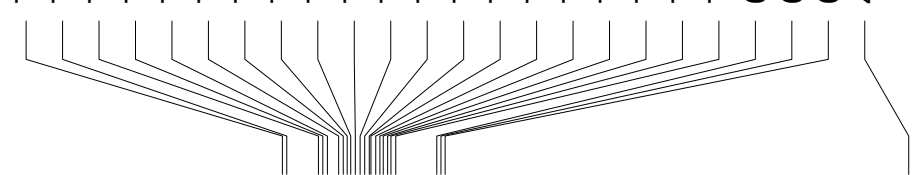

${ }^{1} \mathrm{H}$ NMR $\left(400 \mathrm{MHz}, \mathrm{CDCl}_{3}\right)$

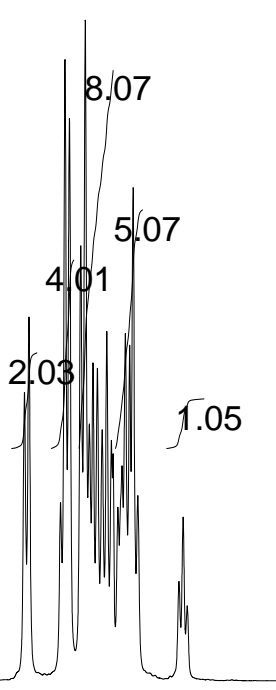

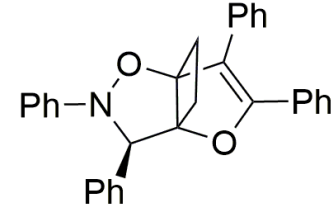

(21) 


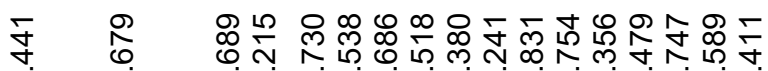

它 守

${ }^{13} \mathrm{C}$ NMR $\left(100 \mathrm{MHz}, \mathrm{CDCl}_{3}\right)$

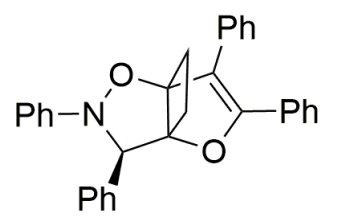

(21) 